\title{
Project Summaries 1996
}
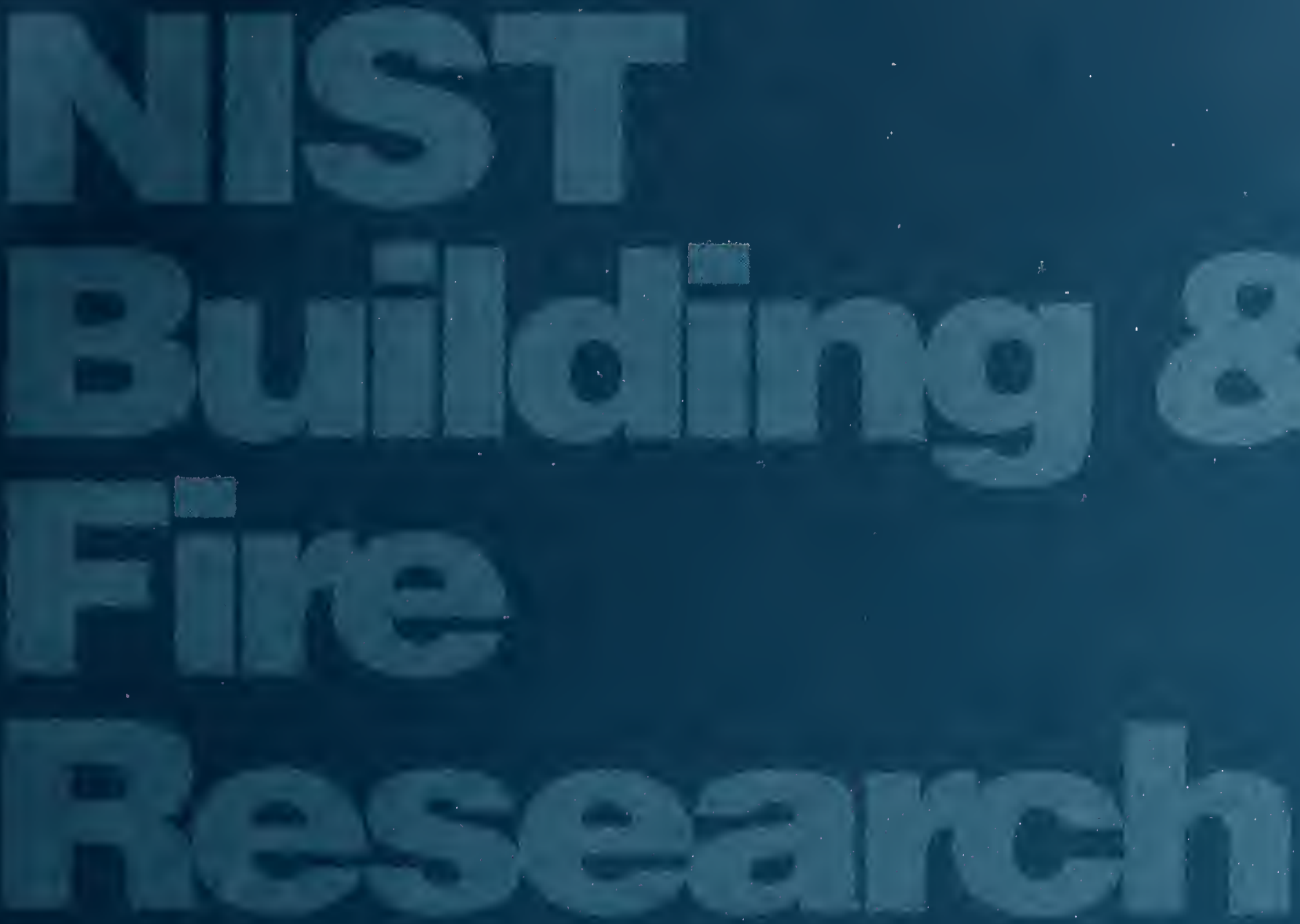

U.S. Department

of Commeree

Technology

Administration

National Institute

of Standards and 



\section{Project Summaries 1996}

NIST SP 838-10
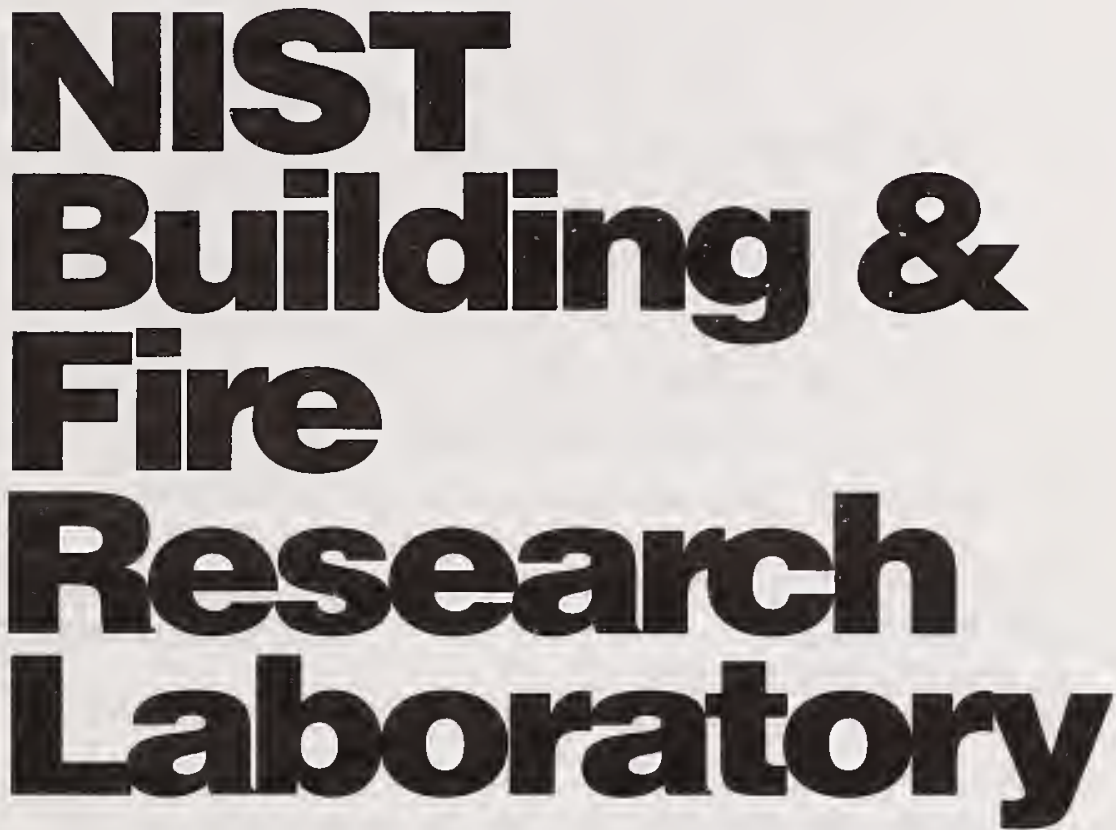

Noel J. Raufaste

September 1996

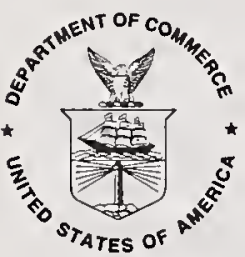

U.S. Department

of Commerce

Michael Kantor, Secretary

Technology

Administration

Mary L. Good,

Under Secretary for Technology

National Institute

of Standards and

Technology

Arati Prabhakar, Director 
National Institute of Standards and Technology

Special Publication 838-10

Natl. Inst. Stand. Technol.

Spec. Publ. 838-10

202 pages (Sept. 1996)

CODEN: NSPUE2
U.S. Government Printing Office Washington: 1996

For sale by the Superintendent of Documents U.S. Government Printing Office Washington, DC 20402 


\section{FOREWORD}

The National Institute of Standards and Technology's (NIST), Building and Fire Research Laboratory (BFRL) is one of NIST's seven Laboratories. The mission of BFRL is to enhance the competitiveness of U.S. industry and public safety through performance prediction and measurement technologies and technical advances that improve the life cycle quality of constructed facilities. Constructed facilities include all buildings and their furnishings and the public and private utilities and public works that support business, commerce, industry, and homes. BFRL's efforts are closely coordinated with complementary activities of industry, professional and trade organizations, academe, and other agencies of government.

Construction is one of the Nation's largest industries. In 1995, total construction amounted to about $\$ 755$ billion which is 12 percent of U.S. GDP (new construction put in place amounted to about $\$ 527$ billion and renovation contributed about $\$ 228$ billion). U.S. construction accounts for more than six million jobs. More than $60 \%$ of the nation's wealth is invested in constructed facilities.

BFRL's research is focused and linked with collaborative private-and public-sector activities to help achieve the National Construction Goals (NCGs) developed with industry by President Clinton's National Science and Technology Council's Subcommittee on Construction and Building (C\&B). This Subcommittee coordinates the federal construction-related $R \& D$ to enhance the competitiveness of U.S. industry, public and worker safety, and environmental quality. BFRL is a cochair of this 16 Federal agency body. During the past 2 years, $C \& B$ has had major interactions with construction industry leaders; together they have defined the NCGs. The goals are to provide costeffective technologies and practices by 2003 that will achieve the following improvements in the life cycle performance of constructed facilities over average 1994 practices:

- 50 percent reduction in delivery time.

- 50 percent reduction in operation, maintenance, and energy costs.

- 30 percent increase in productivity and comfort.

- 50 percent fewer occupancy-related illnesses and injuries.

- 50 percent less waste and pollution.

- 50 percent more durability and flexibility.

- 50 percent fewer construction illnesses and injuries.

This annual report, Project Summaries 1996, presents BFRL's research during 1996. These summaries show how our products (performance prediction, measurement, and test methods) support the industries of construction in achieving the NCGs. BFRL has focused its research in three principal thrusts:

1. High Performance Construction Materials and Systems,

2. Automation in Construction and Constructed Facilities,

3. Loss Reduction.

BFRL's research is in the areas of: structural engineering, materials engineering, mechanical and environmental systems, fire safety and engineering, fire science, and applied economics.

In High Performance Construction Materials and Systems, BFRL's projects include: high performance concrete, building life cycle cost computer program, high performance mechanical systems, finishes, and furnishings, "natural" alternative refrigerants for heat pumps, and 
our search, with industry, for alternative fire suppressants.

Within the automation thrust, research is addressing development of new techniques and standards for real-time construction metrology and a national standard for kinematic modeling of construction site machinery and displaying graphical representations. With U.S. industry, BFRL is developing the capability to represent and exchange information supporting the design and construction of structural systems using internationally accepted protocols. Conformance tests are being developed for products complying with standards for the open exchange of information between equipment from different control vendors and between different levels of control in hierarchal and distributed building management systems.

In the loss reduction thrust, BFRL research includes next generation design standards for wind loads to develop key elements of an improved wind load design standard that will use existing databases and state-ofthe-art computational methods for establishing site-specific design wind speeds and the corresponding steady and fluctuating structural loads. BFRL is constructing a user-friendly version of its CFAST model (FASTLite) which can be used by fire protection engineers and fire service personnel with a minimum of training, and developing protocols to quantify the accuracy of computer-based predictive fire models.

Each of the BFRL research summaries is formatted by project title, the BFRL point of contact, sponsor, description of the research, and recent results.

BFRL's laboratory facilities are an important resource. Facilities include: six-degree-of-freedom structural testing facility; large-scale structural testing facility with the $53 \mathrm{MN}$ (12-million pound) universal structural testing machine; environmental chambers; guarded hotplate; calibrated hot-box; plumbing tower; building materials imaging and modeling laboratory; large burn facility for conducting experimental fires in full-scale and related combustion toxicity facility; large industrial fire test facilities; fire suppression test facilities; and a fire simulation laboratory.

BFRL works closely with its international peer organizations to maintain awareness of foreign research developments, assure that generic research efforts are complementary, and represent U.S. interests in the preparation of international standards and practices. BFRL cooperates closely with other U.S. and foreign laboratories in the conduct of its research. More than 90 research associates from U.S. industry, guest researchers from foreign laboratories, and faculty members from universities worked at BFRL during 1995 for periods averaging about a year. BFRL has direct linkages with industry through its 45 Cooperative Research and Development Agreements (CRADAs).

BFRL participates in more than 130 national and international standardization activities; provides leadership in these national and international standardization organizations and chairs more than 20 voluntary standardization activities. Annually BFRL publishes over 220 reports, articles for research journals and articles for professional and trade journals, and computer model software packages. BFRL staff annually makes hundreds of presentations at professional and technical meetings of building community organizations, is host to more than 1,700 visitors to its facilities, and responds to more than 19,000 requests for information. 
BFRL conducts symposia in cooperation with other organizations concerned with building research and practice and hosts biweekly Fire Research Seminars for NIST staff and colleagues from the fire community. These meetings are effective means of transferring the latest knowledge to practitioners and peer researchers. The Fire Research Information Service (FRIS) consisting of national and international fire research literature and FIREDOC, the automated database of fire research literature, is the only comprehensive national library resource for the fire community.

At the beginning of FY 1996, BFRL had about 217 employees of which 129 are professional staff, 71 have Ph.D.s, and 26 are registered engineers. BFRL's budget for FY 1996 is $\$ 30$ million. Funding comes from direct appropriations ( $\$ 18$ million), from other Federal agencies ( $\$ 10$ million) and from the private sector ( $\$ 2$ million).

For further information about BFRL, its facilities, opportunities for Guest Researcher assignments, collaborative programs, and contracted research, may be reached through BFRL's Home Page at: http://www.bfrl.nist.gov or contact BFRL's Office of Cooperative Research Programs, Building 226, Room B250, NIST, Gaithersburg, MD 20899. Persons requesting information may want to contact BFRL using facsimile number 301 975-4032 or E-mail noel.raufaste@nist.gov. 



\section{CONTENTS}

FOREWORD

\section{EARTHQUAKE ENGINEERING}

Seismic Evaluation of Buried Pipelines ... . 3

Guidelines for Liquid Fuel Distribution

Systems ................. 5

Performance Requirements for Passive Energy Dissipation Systems for Buildings and

Lifeline Structures .............. 6

Seismic Performance of Precast Concrete

Connections ................ 7

Digital Database of Cyclic Lateral Load Tests

on Rectangular R/C Columns ....... 8

Seismic Strengthening Methodologies for

Existing Lightly RC Frame Buildings ..... . 9

Seismic Resistance of Partially Grouted

Masonry Shear Walls ............ 10

Performance of Rehabilitated Masonry

Buildings and Development of Performance-

based Rehabilitation Guidelines . . . . . 12

Seismic Performance of Cladding

Systems $\ldots \ldots \ldots \ldots \ldots \ldots \ldots . \ldots 13$

Seismic Performance of Non-Structural

Ceiling Components ............ 14

Laterial Loads on Manufactured Homes . 15

Management of the Interagency Committee on Seismic Safety in Construction

(ICSSC) ................ 16

Technical Assistance to FEMA Project Officer

(Existing Buildings) $\ldots \ldots \ldots \ldots \ldots \ldots 18$
Technical Assistance to FEMA Project Officer

(New Buildings) ............. 18

Secretariat U.S.-Side Panel on Wind and

Seismic Effects ................ 19

\section{STRUCTURAL ENGINEERING}

Construction Automation ......... 23

High-Performance Concrete: Design and Construction Practices ... . . . . . . . . . 25

Fiber Reinforced Polymer Composite

Structures in Construction ........ 27

High-Performance Steel . . . . . . . . 28

Fire Resistance of High-Strength

Concrete . . . . . . . . . . . . . . . . 29

Next Generation Design Standard for Wind

Loads ..................... . 30

Assessment of Tubular Steel Joints in Existing Offshore Structures ............ 31

\section{COATINGS AND OTHER MATERIALS}

Coatings Service Life Prediction

Consortium ................. 35

Integrated Knowledge System for CorrosionProtective Coatings for High-Performance Steel .................. 36

Database Architectures for the Properties of Construction Materials . . . . . . . . 37

Performance Criteria for Enclosures for Lead-

Based Paint .............. 38 
Performance Criteria for Selection of LiquidCoating Encapsulant for Lead-Based

Paint . . . . . . . . . . . . . . . . . . 39

Method for Evaluation of Performance of Lead-in-Paint Measuring Devices Under Simulated Field Conditions ......... . 40

Factors Affecting the Leaching of Lead from Lead-Based Paint Films . . . . . . . . . 42

Managing Lead-Related Hazards in Housing $. . \ldots \ldots \ldots \ldots \ldots \ldots, \ldots \ldots$

Technical Assistance on Lead-Based Paint Issues ....................44

Performance of Tape-Bonded Seams in Elastomeric Roofing Membranes . . . . . . . 44

International Standards for Design Life of Buildings ................45

\section{CONCRETE}

Computer-Integrated Knowledge Systems for High-Performance Construction

Materials and Systems ............ 49

Computer-Integrated Knowledge Systems for High-Performance Construction Materials and Systems: High-Performance

Concrete .................. 50

Microstructure-Property Relationships in Random Porous Materials ... . . . . . . . 52

Processing of High-Performance

Concrete ..................... 53

Three-Dimensional Microstructural/Kinetic Model of Cement Hydration . . . . . . . 54

Development of Microstructure and

Permeability in Mortar and Concrete . . . 55

Characterization of Clinker, Cement, and Flaws in Cement Paste and Concrete ... 56

Transport Properties of High-Performance Concrete . . . . . . . . . . . . . . . . . 57
Matrix Microcracking in Polymer Composites Under Environmental and Mechanical

Stresses .................. 58

Service Life Prediction of Polymer/Fiber Composites in Civil Engineering Applications

Static and Fatigue Resistance of Polymermatrix Composites Immersed in Simulated Sea Water .................. 60

Spectral Ultra Violet-B Radiation

Database ................... 62

Cementitious Materials Modeling Laboratory

(CMML) . . . . . . . . . . . . . . . . 63

Testing of U.S. Capitol Pavers . . . . . . 63

\section{COMPUTER INTEGRATED CONSTRUCTION}

STEP for the Process Plant Industries . . 67

AMSANT for the Process Plant

Industries $\ldots \ldots \ldots \ldots \ldots \ldots \ldots 68$

Process Plant Engineering and Construction:

Structural Systems . . . . . . . . . . . 69

STEP for the Building Industry . . . . . . 69

Object Oriented Implementation of STEP ................. 70

\section{HEAT TRANSFER}

Development of 2-Dimensional Heat and Moisture Transfer .............. . 73

Test Procedures for Advanced Thermal Insulation Products . . . . . . . . . 74

Low-Temperature and High-Temperature Thermal Insulation Reference Materials . 75 Fibrous-Glass Thermal Insulation SRM . 76 
A Photovoltaic Solar Water Heating

System

\section{INDOOR AIR QUALITY}

Indoor Air Quality Modeling with

Computational Fluid Dynamics

Chemistry and Mass Transport Modeling for Indoor Air Quality . . . . . . . . . . . 82

Development of LAQ Model for

Designers .................. 83

Infiltration and Ventilation in Large

Buildings $\ldots \ldots \ldots \ldots \ldots \ldots . \ldots 4$

Demonstration of Residential Ventilation

Systems ................... 85

Indoor Air Quality Monitoring in Green

Buildings ................ 86

IAQ Analysis of Residential Ventilation

Systems ................. 87

Carbon Monoxide Detector Location in

Residential Buildings . . . . . . . . . 88

\section{MECHANICAL SYSTEMS AND CONTROLS}

Communication Protocols for Building

Controls . . . . . . . . . . . . . . . . . . 93

Real Time Fault Detection and

Diagnostics ...

Test Procedures for Furnaces, Boilers, and Integrated Appliances ............ 95

Commercial Furnace/Boilers, Central Air

Conditioners, Heat Pumps, and Water

Heaters ...................... 97

Test Procedures for Heat Pumps and Air

Conditioners ............... 98

Test Procedures for Lighting Fixtures and Systems
Plumbing Test Procedures 100

Development of Revised Test Procedures for Water Heaters ................ 101

Development of Revised Test Procedures for Ranges and Ovens ............. 102

Development of Revised Test Procedures for Compact Refrigerators ........... 103

Development of Revised Test Procedures for Clothes Washers ... . . . . . . . . . . . 104

Development of Revised Test Procedures for Clothes Dryers ... . . . . . . . . . . . . 105

Development of Revised Test Procedures for Dishwashers . . . . . . . . . . . . . . . . 105

Development of Revised Test Procedures for Pool and Spa Heaters ........... 106

\section{THERMAL MACHINERY}

Thermodynamic Performance of Alternative Refrigerants and Refrigerant Mixtures .. 109

Thermodynamic Performance of Natural Fluids as Alternative Refrigerants ..... 110

Calorimetric and Visual Study of Heat Transfer Enhancements with Alternative Refrigerants .............. III

\section{FIRE MODELING AND APPLICATIONS}

Fire Performance Design of High-

Performance Materials Used in

Construction

BFS Lite ................. 116

Fire Model Evaluation ........... 117

Hazard Version $2 \ldots \ldots \ldots \ldots \ldots \ldots 118$

Hybrid Model Development ........ 119 
Study of Heat and Smoke Movement and Their Influence on Detector and Sprinkler Response in Enclosed Spaces with Complex

Ceiling Geometries ............ 121

Balanced Design ............ 122

Enhanced Fire Safety Evaluation

System ................... 123

Performance Standards . . . . . . . . 123

Fire Safe Elevators ........... 124

Fire Spread in Painted Stairways .... 125

Experimental Application of Fire Hazard

Analysis for U.S. Passenger Train

Systems ................. 126

Fire Research Information Services

(FRIS) . . . . . . . . . . . . 127

Fire Forum . . . . . . . . . 127

Computing and Network Resources ... 128

\section{RECENT GRANTS - FIRE \\ MODELING AND APPLICATIONS}

Interactive Virtual Environments for Fire

Simulation .................. 129

Enhanced PC-based Building Fire Assessment System .................. 130

Accelerating the Application of Advanced Fire Safety Design Methods in the U.S. .... . 131

Evaluation of Alternative Methods for Fire Rating Structural Elements . . . . . . . 132

Computational Heat Conduction for Zone

Fire Modeling . . . . . . . . . . . 134

Flow Through Vents in a Compartment

Fire . . . . . . . . . . . . . . . . . 135

\section{LARGE FIRE RESEARCH}

Fire Flows

Large Eddy Simulation (LES)

Verification ............... 142

Smoke Plume Trajectory From in situ Burning of Crude Oil in Alaska - Phase II . . . . . 143

In-situ Burning of Oil Spills

Demonstration of Water-Based Fire Fighting Agents.................. 144

Demonstration of New Residential

Suppression System Technologies ..... 145

Evaluation of Water Mist System Technology for Light Hazard Occupancy

Applications ............... 146

Office Building Fire Research Program .. 147

Northridge Post-earthquake Fire

Assessment . . . . . . . . . . . . 148

Modification of Fire Protection Requirements for NRC . . . . . . . . . . . . . . . 149

Firefighter Thermal Exposure and the Performance of Protective Clothing in Thermal Environments ........... 150

Development of a Sizing Standard for Firefighter Protective Clothing ...... 151

Large Fire Research Facility

\section{RECENT GRANTS - LARGE FIRE RESEARCH}

Computational Modeling of Large Fire Plumes ... . . . . . . . . . . . . . . 153

A Study of Two Phase High Liquid Loading Jet Fires ................. 154 
Measurement of Flame Temperature Using IR Technique and Air-Entrainment Velocity Using PDPA . . . . . . . . . . . 155

Fire Protection in Large Industrial Fires . 155

A Survey of Occupant Load Factors and Fuel Load in Contemporary Office Buildings . 157

\section{FIRE SENSING AND EXTINGUISHMENT}

Advanced Fire Detection .......... 161

Fire Suppression Chemistry . . . . . . . 162

Low Environmental Impact Fire

Extinguishment ............. 164

Study of Technology for Detecting PreIgnition Conditions of Cooking-Related Fires Associated with Electric and Gas Ranges and Cooktops, Phase II . . . . . . . . . . . . 165

Fire Detection Strategies for High Bays . 166 Analysis of High Bay Hangar Facilities for Detector Sensitivity and Placement ... 167

\section{RECENT GRANTS - FIRE SENSING AND EXTINGUISHMENT}

Detection of Fires Through Reflected

Radiation ................... 168

A Theoretical and Experimental Investigation on Physical, Thermal and Chemical Effects of Condensed Phase Fire Suppressants . . . 169

Investigation of Droplet Penetration Through Complex Openings of Compartments . . 171

\section{MATERIALS FIRE RESEARCH}

Flammability of Structural Composites
Radiative Ignition and Subsequent Flame Spread Over Cellulosic Materials ... . 176

Combustion of a Polymer (PMMA) Sphere in Microgravity . . . . . . . . . . . 177

Chemical Inhibitor Effects on Diffusion Flames in Microgravity ......... 178

Combustion of Silicone ........... 179

Lean Flammability Limit as a Fundamental Refrigerant Property, Phase II ... . . . 180

Calibration Facility for Particle Size and Concentration ............. 181

Burning Rate of Real Materials ...... 182

High Heat Flux Measurement

Standards ................ 183

Evaluation of Fire Barrier Performance . . 184

Furniture Flammability ......... 184

Test Method Development for Cigarette Ignition Propensity ............. 185

Polymeric Film Applied to Glass ... . . 186

\section{RECENT GRANTS - MATERIALS FIRE RESEARCH}

Flame Resistance of Benzocyclobutene (BCB)

Functionalized Copolymers ......... 187

Synthesis, Characterization and Systematic Fire Safety Evaluation of High Volume and Specialty Hydrolytically Stable Phosphate Oxide Containing Polymeric Materials . 188

Modeling of Combustion, Fluid Mechanics, and Radiation in Buoyant Turbulent

Fires .................. 189

Fire Growth Models for Materials .... . 191 
SMOKE DYNAMICS RESEARCH

Carbon Monoxide Production and

Prediction .................... 195

Species Production for Turbulent Flame

Modeling .................. 196

Vortex-Chemistry Interactions in

Methane/Air Diffusion Flames

\section{RECENT GRANTS - SMOKE} DYNAMICS RESEARCH

Mixing and Radiation Properties of Buoyant Luminous Flame Environments ... . . . . 198

Dynamics, Transport and Chemical Kinetics of Compartment Fire Exhaust Gases . . . 200

\section{OFFICE OF APPLIED} ECONOMICS

Economic Support for the NIST Advanced

Technology Program ............. . 205

Measurement of BFRL Research

Impacts . . . . . . . . . . . . . 206

Economics of New-Technology

Materials

Optimal Pricing of Standard Reference

Materials ... . . . . . . . . . . . . . . 208

Baseline Measures for National Construction

Goals 209

Building for Environmental and Economic

Sustainability (BEES)

Life-Cycle Costing Methodology

Provide Economic Support to NIST

Manufacturing Extension Partnership . . 212

Economic Support for the Office of Law

Enforcement Standards . . . . . . . . . . 213
Decision Support Software for Hospitals

Financed by HUD-Guaranteed Loans . . 214

Economic Support for BFRL 


\section{STRUCTURES DIVISION}

EARTHQUAKE ENGINEERING 



\section{Seismic Evaluation of Buried Pipelines}

\author{
Principal Investigators \\ Ronald D. Andrus \\ Structures Division \\ 301-975-6051
}

Bijan Mohraz
Structures Division
301-975-6079

Riley M. Chung

Structures Division

301-975-6062

\section{Sponsor}

National Institute of Standards and Technology

\section{Objective}

To develop procedures for predicting damage to buried pipelines caused by liquefaction, and for seismic analysis, design, and construction of these pipes.

\section{Problem}

Pipelines are vulnerable to large earthquakeinduced ground displacements such as those associated with liquefaction, landslide, slope failure, and fault displacement. Basic types of ground displacement associated with liquefaction include flow failure, lateral spread, transient oscillation, and settlement. To rationally design pipelines, mapping critical areas is necessary. Screening techniques based on geology, hydrology, and soil conditions can identify areas along lifeline corridors requiring more rigorous analyses. These areas can extend for several kilometers, however. Method for rapid evaluation of large areas where such problems may occur is a critical issue.

Further, current computational model used in the design of buried pipes considering soil-pipe interaction lack reliable input data on several critical parameters such as frictional resistance and strains developed at the soil-pipe interface. These parameters and others were developed decades ago by subjecting buried pipes under static load conditions. The validity of these parameters under seismic conditions is very questionable. In addition, there is insufficient information available on materials and response characteristics of older buried pipes. The lack of information, therefore, has become a major concern on how reliably their performance can be predicted or assessed by any analytical method. While many analytical models can simulate pipe performance under seismic loading, none can predict the effect on buried pipes at fault crossings or areas of ground deformation caused by liquefaction.

\section{Approach}

During FY 1996, BFRL will perform two tasks:

1. Develop methods to predict liquefaction and ground deformation potential of soil deposits. This work will be done in three components and in cooperation with industry and academia:

- Apply the system identification technique to the Lotung data made available by EPRI. Research will build on the work started in 1994 to estimate material damping of Lotung soils for the full scale of earthquake-induced strains using the system identification (SI) method. The recording of site response for a full suite of earthquakes at Lotung makes such an analysis possible. Estimates of material damping by the SI method will be compared with published estimates made using laboratory and other analytical techniques. The work will be jointly conducted by BFRL and the Colorado School of Mines.

- Verify the applicability and efficiency of the Spectral-Analysis-of-Surface-Waves (SASW) technique for lifeline applications. This effort builds on the Spring 1996 field demonstration work of the SASW technique for rapid determination of layer thickness and small-strain shear wave velocity. Four sites in the San Francisco Bay area were selected for testing. Testing and initial data reduction jointly will be performed by BFRL and the University of Texas at Austin.

- Perform a feasibility study of the strainenergy approach to liquefaction assessment using shear wave velocity. The strain-energy approach provides a 
more rational framework for deciding the liquefaction potential. Strain energies required to reach various pore water pressures in the laboratory and in the field (the Wildlife liquefaction site) will be compared. Bechtel Corporation, San Francisco is doing this effort, in collaboration with BFRL and the Colorado School of Mines.

2. Examine issues related to soil-pipe interaction under earthquake loading. This work involves four components:

- Conduct a literature survey on performance of pipelines in past earthquakes to decide different modes of failure, and the methods currently used in their design.

- Develop simplified analytical procedures for determining forces and deformation in buried pipes. The analysis will account for soil-pipe interaction, resulting from the various degree of soil deformation around the pipe.

- Design a comprehensive test program to provide information on the critical parameters which are currently lacking, but needed as input for analytical models.

- Conduct limited tests on segments of pipes buried in a container of soil subjected to typical earthquake ground motions on a shake table. The tests include static and dynamic conditions.

Part of the experimental component of the task is expected to be carried out in collaboration with the U.S. Army Construction Engineering Research Laboratory.

It is expected that Tasks 2 will take approximately 3 years to complete.

\section{Recent Results}

Andrus, RD., and Stokoe, K.H., II, "Guidelines for Evaluation of Liquefaction Resistance Using Shear Wave Velocity," Proceedings, NCEER Workshop on Evaluation of Liquefaction Resistance, January 1996.

Glaser, S. D. and Chung, R. M., "Use of Ground Improvement for Liquefaction Mitigation Potential for Lifelines," Proceedings, U.S.-Taiwan Geotechnical Collaboration Workshop,
Taipei, Taiwan, 1995.

Andrus, R. D. and Chung, R M., "Costeffective Ground Improvement Techniques for Liquefaction Remediation Near Lifelines," Proceedings, 27th Joint Meeting of the U.S.-Japan Cooperative Program in Natural Resources Panel on Wind and Seismic Effects, Technical Memorandum No. 3387, Public Works Research Institute, Japan 1995.

Andrus, RD., "Surface Wave Method - A Tool for Lifeline Earthquake Engineering," Proceedings, Earthquake Engineering Site Characterization Workshop, U.S. Army Corps of Engineers, Vicksburg, Mississippi 1995.

Andrus, R. D. and Chung, R. M., Ground Improvement Techniques for Liquefaction Remediation near Existing Lifelines, NISTIR 57 14, National Institute of Standards and Technology, October 1995.

Glaser, S. D., and Chung, R.M., "Estimation of Liquefaction Potential by In Situ Methods," Earthquake Spectra, EERI, Vol.11, No.3, 1995.

Glaser, S. D., "Surface Displacements due to Earthquake Excitation for Saturated Sands," Earthquake Spectra, EERI, Vol. 10, No. 3, 1994.

Glaser, S. D., "Application of Parametric Models for Estimation of Soil Parameters," Proceedings, 8th International Conference of the Association for Computing Methods and Advances in Geomechanics, Vol. 1, 1994.

Glaser, S.D., and Chung, R.M., "Evaluation of Liquefying Soil Through Time Using System Identification," Proceedings, 5th U.S.-Japan Workshop on Earthquake Resistant Design of Lifeline Facilities and Countermeasures Against Soil Liquefaction, Technical Report NCEER-94-0026, NCEER, 1994. 


\section{Guidelines for Liquid Fuel Distribution Systems}

\section{Principal Investigators}

Bijan Mohraz

Structures Division

301-975-6079

Riley M. Chung

Structures Division

301-975-6062

\section{Sponsor}

National Institute of Standards and Technology

\section{Objective}

To develop guidelines for the seismic design and construction of liquid fuel distribution systems.

\section{Problem}

While there was no severe damage to liquid fuel systems; storage tanks, connections, buried and elevated piping, during recent earthquakes such as the 1994 Northridge, California and the 1995 Kobe, Japan earthquakes, the potentials for devastating effects can occur, such as damage to LNG tanks and to major trunk lines. For instance, a major Midwest earthquake may cause severe damage to liquid fuel distribution lines that provide heating fuels for residents in the New England area. When this happens in the winter, it results in major spills in the vicinity where the pipes break and creates hardship, perhaps life losses in the New England region from loss of heating fuel. Most of the existing systems, especially those found east of the Rocky's, were designed with no or minimum seismic consideration. It is important that this problem be addressed to determine their vulnerability and provide guidance how existing systems should be strengthened and new systems designed.

\section{Approach}

During FY 1996, BFRL will perform two tasks:

1. Assess seismic risks of liquid fuel systems.

This work is to review and integrate available methods and procedures of seismic risk assessment and loss estimation, develop a framework for risk assessment that can logically accommodate the state of the art results of BFRL work addressing the physical and functional performance of the liquid fuel systems subject to earthquakes, identify and highlight the design issues that must reflect the risk concept during the development of design guides, and develop and draft an outline of design guides. BFRL is conducting this work through the University of Southern California.

2. Evaluate the seismic performance of liquid fuel tanks. This effort involves the documentation and evaluation of the performance of liquid fuel tanks during the past and recent major earthquakes, assess their performance with respect to the current design and construction practices, and develop recommendations for improving their future performance.

This project is partially to support a National Lifeline Plan developed by NIST and FEMA, with participation from the private sector, that has been submitted to Congress by FEMA. The Plan recommends the establishment of a Lifeline Seismic Safety Executive Board to coordinate and execute projects aimed at developing design and construction guidelines for lifeline systems including the liquid fuel systems.

\section{Recent Results}

New project 


\section{Performance Requirements for Passive Energy Dissipation Systems for Buildings and Lifeline Structures}

\author{
Principal Investigators \\ Andrew Taylor \\ Structures Division \\ 301-975-6078 \\ Riley M. Chung \\ Structures Division \\ 301-975-6062
}

Sponsor

National Institute of Standards and Technology

\section{Objective}

To develop and verify guidelines for prequalification, prototype and quality of seismic isolation systems.

\section{Problem}

Seismic isolation has been proven to be effective in reducing the level of response in structures during strong earthquake ground shaking. In practice, the components of the isolation system undergo an extensive series of prototype and quality control tests before installation.

Recently BFRL completed an extensive set of guidelines for conducting these tests. These guides were developed with the assistance of an Oversight Committee of five experts from the field of seismic isolation. They were refined to reflect the comments received from about 40 reviewers, and the recommendations developed at a BFRL workshop in July 1994. To develop confidence in the guidelines, and to foster wider adoption of the proposed testing procedures, test must be conducted on typical isolation components to evaluate the guidelines. These tests are needed to expose inconsistencies, omissions of important data, or other unforeseen problems with the procedures.

\section{Approach}

During FY 1996, BFRL will perform three tasks:

1. Publish the Final BFRL Guidelines. Incorporate into the draft guidelines the final review comments and recommendations from mail-in reviews, BFRL workshop, and the Oversight
Committee. This document will serve as the pre-standard document for development of the ASCE national consensus standard.

2. Support Development of a National Consensus Standard. As an ongoing component of this project, BFRL will provide the secretariat of the ASCE standard's committee on testing of base isolation systems. The committee was formed in August of 1995, and the first meeting was held in September. Subsequent meetings will take place every 6 months, for approximately 2 years.

3. Test Program for Assessing and Evaluating Guidelines. A detailed plan has been developed for the experimental program, through consultations with researchers at the University of Delaware, the University of Maryland, and the Southern Methodist University. The objectives of the experimental program will be to evaluate the NIST testing guidelines, and study three other issues which affect test methods: the ultimate capacity of seismic isolators; the effects of bilateral loading on isolator performance; and improved methods for detecting flaws in elastomeric bearings. During FY 1996, 23 isolators will be evaluated in two phases. The first phase of tests will involve primarily uniaxial compression loading and is planned for early FY 1996. The second phase will involve testing isolators under simultaneous uniaxial compression and cyclic lateral shear loading. These tests will be conducted in the BFRL Tri-Directional Testing Facility (TTF) and possibly its $53 \mathrm{MN}$ capacity universal testing machine.

\section{Recent Results}

Shenton, H.W., and Taylor, A.W., "Guidelines and Benchmarks for Analysis of Isolated Buildings," Proceedings, ASCE Structures Congress, Chicago, April 1996.

Shenton, H.W., Summary and Results of the NIST Workshop on Proposed Guidelines for Testing and Evaluation of Seismic Isolation Systems, July 25, 1994, San Francisco, Ca, NISTIR 5785, National Institute of Standards and Technology, January 1996. 
Shenton, H.W., Guidelines for Pre-Qualification, Prototype, and Quality Control Testing of Seismic Isolation Systems, NISTIR 5800, National Institute of Standards and Technology, January 1996.

Taylor, A.W., Shenton, H.W., and Chung, R.M., "Standards for Testing and Evaluation of Seismic Isolation Systems," Seismic, Shock, and Vibration Isolation, ASME/SSME PVP Conference, Honolulu, July 1995, PVP-Vol 319, pp. 39-43, 1995.

Shenton, H.W., Taylor, A.W., and Lew, H.S., "Test Requirements for Base Isolation," Proceedings, 27th Joint Meeting on Wind and Seismic Effects, U.S.-Japan Panel on Wind and Seismic Effects, UINR, Technical Memorandum No. 3387, Public Works Research Institute, Japan, May 1995.

\section{Seismic Performance of Precast Concrete Connections}

\author{
Principal Investigator \\ Geraldine S. Cheok \\ Structures Division \\ 301-975-6074
}

\section{Sponsor}

National Institute of Standards and Technology

\section{Objective}

To revise the design guidelines for moment resistant precast concrete beam-column connections for adoption into building codes and standards.

\section{Problem}

Proposed revisions to the building codes require careful attention to the content and wording of the proposed changes. Attention is required to avoid conflicts with existing code requirements, the unintentional exclusion of materials and/or procedures, and potential mistakes caused by misinterpretation of the proposed changes.

\section{Approach}

During FY 1996, BFRL will coordinate its research with private sector consulting engineers and academic researchers. The proposed changes will be presented to the SEAOC Ad Hoc Committee on Precast Structures on Seismic Zones. The Committee will provide guidance to the technical content and acceptability of the changes by the SEAOC Seismology Committee. Interaction with the ad hoc committee will include participation in the scheduled meetings to provide technical interpretation of the proposed design guidelines. To verify a procedure proposed in the recommended guidelines, BFRL researchers will collaborate with a precast construction company in processing data from additional tests sponsored and conducted by the precast company.

Tests of the horizontal connections for precast panels will be completed and a report will be published. Recommendations for vertical and horizontal connections for precast panels will be submitted to ACI Technology Transfer 
Committee (TTC) for their consideration as a demonstration project. Subsequently, a proposal for design provisions will be made by ACI TTC for inclusion in ACI 318 Building Code.

\section{Recent Results}

Cheok, G.S., Stone, W.C., and Nakaki, S.P., Simplified Design Procedures for Hybrid precast Concrete Connections, NISTIR 5765, National Institute of Standards and Technology, February 1996.

Stone, W.C., Cheok, G.S., and Stanton, J.F., "Performance of Hybrid Moment-Resisting Precast Beam-Column Concrete Connections Subjected to Cyclic Loading," ACI Structural Journal, Vol.92, No.2, American Concrete Institute, Detroit, MI, pp. 229-249, MarchApril 1995.

Cheok, G.S., and Stone, W.C., Performance of 1/3-Scale Model Precast Concrete Beam-Column Connections Subjected to Cyclic Inelastic Loads Report No.4, NISTIR 5436, National Institute of Standards and Technology, June 1994.

Cheok, G.S., Stanton, J.F., and Seagren, D., "Beam-to-Column Connections for Precast Concrete Moment-Resisting Frames," Proceedings of the Fourth Joint Technical Coordinating Committee on Precast Seismic Structural Systems, May 1994.

\section{Digital Database of Cyclic Lateral Load Tests on Rectangular R/C Columns}

\author{
Principal Investigators \\ William C. Stone \\ Structures Division \\ 301-975-6075 \\ Andrew Taylor \\ Structures Division \\ 301-975-6078
}

\section{Sponsor}

National Center for Earthquake Engineering Research (NCEER)

\section{Objective}

To develop a comprehensive digital data base of cyclic lateral load tests on rectangular reinforced concrete columns.

\section{Problem}

To develop confidence in inelastic analysis algorithms for reinforced concrete structures, the algorithms must be calibrated against laboratory data from tests on reinforced concrete members. Without such a calibration, the inelastic analysis algorithms are essentially useless. Because of the high variability inherent in tests of reinforced concrete members, and because of the wide range of member geometries and material properties covered by the analytical models, large databases of experimental results are required for adequate calibrations. There is currently no comprehensive, worldwide database of results from cyclic lateral load experiments on rectangular columns

\section{Approach}

During FY 1996, BFRL will develop a comprehensive digital database of cyclic lateral load tests on rectangular reinforced concrete columns. This work will build on the past year's effort involving a literature review that identified worldwide cyclic lateral load tests and copies of test reports and publications on digital data. For those tests which are reported only in analog format, the load-deflection plots will be digitized using a computer work station, 
digitizing tablet and special digitization software written expressly for this purpose at BFRL. Finally, the digital data will be arranged in a uniform format, auxiliary information about the tests will be tabulated, and the database will be described in a report accompanied by a computer disk.

\section{Recent Results}

Completed collecting laboratory records on tests of rectangular columns.

\section{Seismic Strengthening Methodologies for Existing Lightly RC Frame Buildings}

\author{
Principal Investigators \\ Geraldine S. Cheok \\ Structures Division \\ 301-975-6074
}

Long Phan

Structures Division

301-975-6077

\section{Sponsor}

National Institute of Standards and Technology

\section{Objective}

To develop guidelines for strengthening lightly reinforced concrete (LRC) frames using infill walls.

\section{Problem}

A workshop conducted by NIST on the seismic rehabilitation of LRC frames in FY 1995 identified research needs in this area. Several issues were categorized as high priority. One issue addressed the prediction of the behavior of LRC frames, unstrengthened and strengthened. Accurate prediction of frame behavior would allow for rational decisions on whether to rehabilitate or not and the most appropriate rehabilitation method. How well the connections perform their intended purpose of load transfer influences the success of any rehabilitation method. Currently, the design of connections is based on shear friction theory which is conservative for this application and typically results in the requirement for a great many connectors. There is an economic incentive to rationally determine the required amount of connectors.

\section{Approach}

During FY 1996, BFRL will develop analytical methods to predict the behavior of LRC frames strengthened with infill walls. Fundamental to such methods is the ability to model the behavior of the connectors accurately. The development of a relationship to allow for the accurate prediction of connector behavior would therefore enhance the reliability of current 
analytical methods. The development of this relationship will require several years and will involve:

1. Determination of available data on the load-deformation relationship up to failure of typical connectors used for connecting new elements to existing LRC frames.

2. Development of an empirical load deformation relationship of the connectors based on parameters including connector diameters, concrete strength, embedment depth, etc.

3. Determination of available finite element analysis packages where the connector relationship, developed in the previous step, can be implemented to enable the determination of the inelastic behavior of LRC frames up to failure. Modifications may have to be made to the program to fulfill the stated objective.

4. Parametric study using the finite element program.

5. Experimental tests to verify the validity of the analytical results.

\section{Recent Results}

Cheok, G. S., Editor, Proceedings, Workshop on the Seismic Rehabilitation of Lightly Reinforced Concrete Frames, NISTIR 5741, National Institute of Standards and Technology, November 1995.

Phan, L. T., Cheok, G. S., Todd, D. R and Lew, H. S., Strengthening Methodology for Lightly Reinforced Concrete Frames: Recommended Design Guidelines for Strengthening with Infill Walls, NISTIR 5682, National Institute of Standards and Technology, July 1995.

Phan, L. T., Todd, D. R, and Lew, H.S., Strengthening Methodology for Lightly Reinforce Concrete Frames - II. Recommended Calculation Techniques for the Design of Infill Walls, NISTIR 5421, National Institute of Standards and Technology, May 1994.

\section{Seismic Resistance of Partially Grouted Masonry Shear Walls}

Principal Investigators

Gerry Cheok

Structures Division

301-975-6074

Arturo E. Schultz

Structures Division

310-975-5301

\section{Sponsor}

National Institute of Standards and Technology

\section{Objective}

To define the strength and deformation of partially grouted masonry shear walls at the cracking and ultimate limit states, and the influence of critical parameters on wall behavior.

\section{Problem}

The behaviors of masonry shear walls have been the subject of increased attention during the past fifteen years. However, effort has been aimed at fully grouted, reinforced walls. Due to the lack of physical data on the response of partially grouted masonry walls to in-plane lateral loads, no acceptable design formulas are available for predicting shear strength of such walls. The limited data available also suggests that empirical formulas for shear strength of fully grouted, reinforced walls have very limited applicability for determination of the shear strength of partially grouted, lightly reinforced shear walls.

The tests and analyses conducted by BFRL in FY 1995 for the reinforced bond beams, showed that the load resisting mechanism in partially grouted walls is considerably different from that for fully-reinforced and fully grouted walls. Cracking of the intersection between bond beams and grouted vertical cells may be the controlling mechanism for wall resistance, as it limits how much stress that horizontal reinforcing bars can carry.

\section{Approach}

During FY 1996, BFRL will evaluate the second 
series of specimens of highest priority, which target the replacement of bond beams with special (ductile) bed joint reinforcement. This series of experiments will be conducted on partially grouted masonry shear walls made using hollow concrete units with six specimens. The research will study the amount of horizontal reinforcement and aspect ratios on shear behavior of walls in which all horizontal reinforcement is provided by welded grids placed in the bed joints. Horizontal reinforcement ratios equal to 0.0005 and 0.0012 are incorporated in the experimental program, with aspect ratios of $0.5,0.7$ and 1.0. These specimens are nominally identical to those in Series II (tested and analyzed in FY 1995) except the replacement of bond beams with bed joint reinforcement.

Analytical activities will be conducted in parallel with the experiments, and these include development of semi-empirical simplified expressions for predicting cracking and ultimate shear strengths and deformations. Similar efforts at NIST have led to the improvement of a shear strength formula for fully-grouted, reinforced masonry shear walls. The behavior of partially grouted masonry shear walls using a more systematic procedure that can give global and local information regarding states of stress and strain at cracking and yielding will be performed. For this purpose, two levels of finite element analysis of partially grouted walls are used. A nonlinear finite element computer package for masonry shear walls (FEM) will be use to verify the applicability of nonlinear finite element analyses for determining peak strength and initial stiffness of partially-grouted masonry walls.

Research results from this study will be submitted to the Masonry Standards Joint Committee (MSJC) and Building Seismic Safety Committee (BSSC) for possible incorporation in the ACI-ASCE-TMS recommended building code provisions and the NEHRP (National Earthquake Hazard Reduction Program) suggested guidelines provisions, respectively.

\section{Recent Results}

Schultz, A. E., "Minimum Horizontal
Reinforcement Requirements for Seismic Design of Masonry Walls," TMS Journal, The Masonry Society, (for publication).

Schultz, A.E., "Seismic Resistance of Partially Grouted Masonry Shear Walls," 11 th World Conference on Earthquake Engineering, Acapulco, Mexico, June 1996.

Schultz, A. E., NIST Research Program on the Seismic Resistance of Partially-Grouted Masonry Shear Walls, NISTIR 5481, National Institute of Standards and Technology, June 1994. 


\section{Performance of Rehabilitated \\ Masonry Buildings and \\ Development of Performance-based \\ Rehabilitation Guidelines}

\author{
Principal Investigator \\ Riley Chung \\ Structures Division \\ 301-975-6062
}

\section{Sponsor}

Federal Emergency Management Agency

Mitigation Directorate

\section{Objective}

To document the performance of rehabilitated unreinforced masonry buildings during the Northridge earthquake, evaluate the effectiveness of current rehabilitation practices, and develop a compendium of rehabilitation techniques with guidance for designers.

\section{Problem}

Reinforced masonry construction has been used in California since 1934 after the Long Beach earthquake. However, many unreinforced masonry buildings (URMs) still exist in the Los Angeles area. Many of these buildings have been "rehabilitated" as the result of the rehabilitation requirements known as "Division 88" mandated by the City of Los Angeles. The requirements apply to all URMs other than one and two-family dwellings and small apartment buildings of less than five living units.

Despite rehabilitation, many URMs in Santa Monica and in Hollywood were heavily damaged as the result of the January 1994 earthquake. As a life-safety measure, current rehabilitation practices appear to have been successful in this earthquake--there were no complete collapses and no deaths of occupants. In contrast, a significant portion of the fatalities due to building failures during the 1989 Loma Prieta earthquake were the result of out-ofplane failures of URM walls that collapsed onto adjacent buildings, sidewalks, streets, and parking lots. Yet, the Northridge earthquake occurred at 0431 making it unlikely for deaths or injuries to occur on the sidewalks adjoining the damaged URMs. Furthermore, rehabilitation was not successful in preventing property damage, which can lead to significant economic losses as the result of building repair and the associated business disruption.

The Northridge earthquake offers an opportunity to examine the effectiveness of the current rehabilitation practices as exemplified by the implementation of "Division 88." There are many rehabilitated URMs that this earthquake affected. There is a need to systematically develop a database that is as complete as possible to document the locations of these URMs and their performance. The database will be developed from building department records rather than from site visits of individual buildings. The earthquake also resulted in many strong motion records throughout the region. Comparison of URM location and performance to the characteristics of local strong motion records, and to traditional MMI Intensity maps, will allow a comprehensive evaluation of the effectiveness of current rehabilitation requirements.

\section{Approach}

During FY 1996, BFRL will develop a compendium of rehabilitation techniques, for all levels of performance. Information gaps will be identified. This document will provide immediately usable information for designers, and will complement BFRL's work for FEMA in development of rehabilitation guidelines for multiple performance objectives for all building types by 1997 . The work is being performed in collaboration with outside investigators.

\section{Results}

New project 


\section{Seismic Performance of Cladding Systems}

\author{
Principal Investigators \\ Andrew W. Taylor \\ Structures Division \\ 301-975.6078
}

\section{Sponsor}

National Institute of Standards and Technology

\section{Objective}

To evaluate seismic performance of exterior architectural cladding elements during the Northridge earthquake and develop energy dissipating cladding systems for seismic retrofit and design of new buildings.

\section{Problem}

Although many cladding elements are not specifically designed for seismic forces, they participate in resisting lateral loads as they deform with the framing system. It has been reported that cladding systems sustained damage during the Northridge earthquake, particularly those on steel frame structures. The seismic performance of buildings could be improved by utilizing effectively the cladding system to dissipate energy. Energy-dissipating cladding can be applied to both new construction and seismic retrofit.

\section{Approach}

During FY 1996, BFRL will document the performance of architectural cladding systems during the Northridge and other earthquakes. Current design criteria and code requirements will be critically evaluated based on observed cladding performance. Next, the performance of energy dissipating cladding connector hardware will be studied. This will be primarily an experimental investigation, using existing test fixtures at Georgia Institute of Technology. The goal of this work will be to determine how different types of energy dissipating connectors behave (e.g., elastomeric connectors, steel clip angles, and other specially designed steel connectors). The hysteretic properties of the connectors will be established, and desirable modes of connector performance will be defined. Following this effort, the contributions of cladding to the stiffness and damping of the overall structural system will be determined. Presently, it is not clear to what degree cladding systems participate with the main structural frame of a building during earthquake shaking. This work follows naturally on the connector performance studies described above, since the energy dissipating properties of connectors have a major influence on the interaction between the cladding system and the structural frame. The third project task will be to compare the full-scale and scale-model building performance data with analytical models. Finally, based on the results of the above work, seismic design guidelines for building cladding systems will be developed.

\section{Recent Results}

Cladding Research Institute, Literature Review on Seismic Performance of Building Cladding Systems, GCR-95-681, National Institute of Standards and Technology, February 1995. 


\section{Seismic Performance of Non- Structural Ceiling Components}

\author{
Principal Investigators \\ Long T. Phan \\ Structures Division \\ 301-975-6077 \\ Andrew Taylor \\ Structures Division \\ 301-975-6078
}

\section{Sponsor}

National Institute of Standards and Technology

\section{Objective}

To develop methods for reducing seismic damage to non-structural components of buildings.

\section{Problem}

It has been observed in past earthquakes that damage to non-structural components and secondary systems can have a great effect on the safety of occupants and loss of property, and sometimes the effects of non-structural damage can be even greater than the effects of structural damage. The terms "non-structural components" and "secondary systems" refer to a number of items. They include, for instance, cladding, ceilings, building contents, equipment, and IVAC, plumbing, and electrical systems. Most of the past efforts in the NEHRP program have focused on improvements of the structural design of buildings to prevent total collapse. As a result, most newly constructed buildings stand a good chance that they will not collapse from an anticipated earthquake. It is only recently that attention has been paid to the performance of non-structural components and secondary systems.

A major problem observed in all recent major U.S. earthquakes has been the seismic performance of the components located in or above suspended ceilings in commercial office buildings. These components include the suspended acoustical tile ceilings themselves; fire sprinkler systems; light fixtures; and HVAC ducts. Problems arise because these components are co-located in the ceiling area, and their movements during an earthquake are often incompatible. For example, fire sprinkler heads usually project through suspended acoustical tile ceilings. During an earthquake, the movements of the suspended ceiling and the sprinkler pipes are incompatible, so that the sprinkler heads are damaged. Not only does this decrease the ability of the sprinkler system to suppress post-earthquake fires, but it also may result in broken sprinkler heads and flooding of the building contents. Another example is distortion of the acoustical tile ceiling grid, which may cause lighting fixtures and ventilation grills resting in the grid to fall.

Thus, studies of the interactions of components located in and above suspended ceilings are required to reduce non-structural damage in buildings. Design guidelines need be developed to ensure compatible deformations and adequate seismic resistance of these components.

\section{Approach}

During FY 1996, BFRL will perform a literature review; analytical modeling; and shake table testing. The study will be initiated with a review of the seismic performance of nonstructural building components, and the stateof-the-art of seismic design methods for nonstructural components. This will be followed by a search for existing literature on seismic behavior of non-structural components in ceilings (pipes, HVAC ducts, suspended ceilings).

Computer models will be evaluated which could be used to perform dynamic analyses of ceiling systems. These models would include provisions for real-time opening and closing of gaps between components; realistic component flexibility; and possibly progressive damage of components. Initial modeling would be in two dimensions (one horizontal axis and the vertical axis), and later modeling might be extended to three dimensions.

Once a satisfactory modeling method has been established, realistic ceiling component data will be required. These include component masses, stiffness and strengths, the layout of components in the ceiling area, and appropriate 
input seismic records. Some simple testing of typical components, such as fire sprinkler pipes, may be required to obtain stiffness data.

Several typical geometric layouts of ceilings system will be defined, and three to five input earthquake records of varying magnitudes. Using the forces and differential displacements will be recorded. Observed problem areas, such as impact sites and locations of high stress will be studied, and remedial measures will be proposed. Alterations will be made to the computer model to remedy the problem areas, and the computer analyses will be repeated to assess the degree of improvements.

Based on the analytical studies, recommendations will be made for improving the seismic design of components within and above suspended ceilings in buildings. The recommendations include minimum or maximum clearances between components; strengths for anchorages; and fixing some components or for freeing other components.

The analytical phase of the project will be followed by an experimental phase, expected to commence in FY 1997. The purpose of the experimental phase is to verify the analytical model, and to evaluate the recommended design procedures developed earlier in the project. In the experimental phase, a full scale model of a typical ceiling and plenum area will be constructed on a shaking table. The model will include the suspended ceiling system, pipes (fire suppression, water, sewer, gas), and HVAC ducts. This model will be subject to a variety of earthquake motions, and damage to the model components will be observed. The model will then be altered according to the design procedures developed earlier, to determine the extent to which these practices improve the dynamic behavior of the model. The design guidelines will be modified as necessary to reflect the findings of the experimental program.

\section{Recent Results}

Phan, L.T. and Taylor, A.W., State of the Art Report on Seismic Design Requirements for Nonstructural Building Components, NISTIR 5857. National Institute of Standards and Technology, June 1996.

\section{Lateral Loads on Manufactured Homes}

\section{Principal Investigator}

R. D. Marshall

Structures Division

301-975-6071

\section{Sponsor}

National Institute of Standards and Technology

\section{Objective}

To develop performance-based requirements and recommended procedures for the design of manufactured home anchoring and support systems to resist lateral forces.

\section{Problem}

The Northridge Earthquake of January 17 , 1994 again showed the vulnerability of manufactured homes to strong ground motions. Most of the damage to manufactured homes involved the shifting or lateral movement of homes about their supporting piers so that the piers either jackknifed or punched through the floor system. In several instances the lateral movement was sufficient to rupture gas lines, or gas lines were ruptured as the homes fell from their supporting piers. In one manufactured home park, at least six individual ignitions of leaking natural gas occurred, and the resulting fires consumed several nearby units. Many affected homes were equipped with secondary support systems whose function is to catch the manufactured home and thus reduce damage when primary support is lost. However, these secondary systems frequently lacked sufficient lateral bracing to resist earthquake forces.

Although it is well established that wind loading will dictate the strength requirement for manufactured home anchoring systems in most situations; earthquake loading subjects the various elements of these systems to many cycles of reversed loading in the horizontal and vertical directions. Frictional forces and the lateral resistance of supporting piers mobilized under wind loading cannot be relied upon in an earthquake. To predict anchorage and support system performance under earthquake loading, the stiffness, ductility, energy dissipation and 
softening characteristics of these systems must also be known. To date, little attention has been given to this problem so that the required databases from which to formulate adequate design criteria is nonexistent.

\section{Approach}

During FY 1996, BFRL will:

1. Develop a computer-based model to perform sensitivity studies of traditional anchorsupport systems. The model will accommodate various distributions of superstructure dead load and stiffness, anchorage and pier stiffness, and ductility of chassis-to-pier connections. Ground motion input will range from constant-amplitude cyclic displacements to actual strong-motion time histories.

2. Develop envelopes of reaction forces (piers, pier-to-frame connections, frame-to-anchor ties, and soil anchors) as a function of ground acceleration, damping, and system mass and stiffness.

3. Develop typical load time-histories for the components identified under Item 2 above.

\section{Recent Results}

New project

\section{Management of the Interagency Committee on Seismic Safety in Construction (ICSSC)}

\author{
Principal Investigator \\ Ann Bieniawski \\ Structures Division \\ 301-975-6065
}

\section{Sponsor}

Federal Emergency Management Agency Mitigation Directorate

\section{Objective}

To facilitate and expedite the work of the Interagency Committee on Seismic Safety in Construction (ICSSC), by providing the Chair and Technical Secretariat.

\section{Problem}

The National Earthquake Hazard Reduction Program (NEHRP) established the ICSSC to assist Federal departments and agencies develop, improve, and maintain seismic safety programs for all types of construction. Thirty Federal departments and agencies currently participate in the ICSSC. The National Earthquake Hazard Reduction Act (amended by Public Law 101-614) designates the director of NIST, or his deputy, as the chair of the ICSSC. BFRL provides the Secretariat to the ICSSC where it facilitates, documents, and disseminates the work of the committee.

The ICSSC is developing implementation guidance for Executive Order 12941, Seismic Safety of Existing Federally Owned or Leased Buildings. Section 2 of the Order requires all building-owning agencies to develop a seismic inventory and an estimate of the cost of achieving adequate seismic safety in their buildings. The information collected on the Federal government's half-million owned buildings will be used to examine potential rehabilitation programs, and to recommend to Congress an economically feasible way to upgrade the seismic safety of the Federal building population. The ICSSC guidance is aimed at ensuring that the data collected by the agencies is consistent in content and format, so an effective seismic rehabilitation program, 
based on reliable and defensible data, will be proposed to Congress.

\section{Approach}

During FY 1996, BFRL serving as the ICSSC

Technical Secretariat, will continue to perform the day-to-day management of the full committee and the five subcommittees by organizing and documenting meetings, maintaining rosters, distributing information. The ICSSC will encourage consistency of implementation of EO 12941 by:

1. Developing cost estimating tools and methodologies. Submitted data will be used to assess vulnerability of many building types. The Second Edition - Typical Costs cost estimating methodology will be adapted for use in subdividing cost estimates into the cost reporting categories called for in the ICSSC guidance. Other potential tools and methodologies that could aid the agencies will be identified and developed.

2. Holding twice-yearly workshops to provide a forum for agencies to share successes and difficulties in implementing the order. Agency personnel will be trained in the use of the ICSSC-developed methodologies.

3. Providing additional agency-specific technical support upon request, as explanation, interpretation and/or training in the use of the ICSSC guidance documents, distribution of ICSSC documents, presentations at meetings, review and comment on proposed methodologies, and similar efforts.

4. Naming a Database Manager, who will identify and acquire needed hardware and software for the government-wide database, develop the basic framework for the database, and begin working with agency personnel to test protocols for transmitting data.

\section{Recent Results}

Bieniawski, A., and Todd, D., How To

Suggestions for Implementing Executive Order 12941 on Seismic Safety of Existing Federal Buildings, NISTIR 5770, ICSSC TR17, National Institute of Standards and Technology, November 1995.

Todd, D., and Bieniawski, A., Editors, ICSSC Guidance on Implementing Executive Order 12941 on Seismic Safety of Existing Federally Owned or Leased Buildings, NISTIR 5734, ICSSC RP 5, National Institute of Standards and Technology, October 1995.

Todd, D., Seismic Safety of Federal Buildings Initial Program: How Much Will It Cost?, NISTIR 5419, National Institute of Standards and Technology, April 1994.

Todd, D., Editor, Standards of Seismic Safety for Existing Federally Owned or Leased Buildings, NISTIR 5382, ICSSC RP 4, National Institute of Standards and Technology, February 1994. 


\section{Technical Assistance to FEMA Project Officer (Existing Buildings)}

\author{
Principal Investigator \\ Ann Bieniawski \\ Structures Division \\ 301-975-6065
}

\section{Sponsor}

Federal Emergency Management Agency

Mitigation Directorate

\section{Objective}

To provide the technical review of FEMAfunded projects aimed at developing design and construction guidance documents for seismic rehabilitation of existing buildings.

\section{Problem}

FEMA supplements the capabilities of their staff by using the technical expertise of BFRL personnel.

\section{Approach}

During FY 1996, BFRL will review statements of FEMA work and requests for proposals; participate on proposal evaluation panels, in advisory panel meetings, in users' workshops, and selected technical seminars and meetings; and review draft and final project products, providing verbal and written comments. During 1996, FEMA requested technical assistance in the following:

- "Guidelines for Seismic Rehabilitation," and supporting documentation, being prepared by BSSC/ATC/ASCE;

- "Plan 2005," development of a strategy for improving seismic rehabilitation tools between 1996 and 2005;

- a homeowner's guide to seismic rehabilitation; and

- an update of FEMA 178, the NEHRP Handbook for the Seismic Evaluation of Existing Buildings.

\section{Recent Results}

No published results.

\section{Technical Assistance to FEMA Project Officer (New Buildings)}

\author{
Principal Investigators \\ H.S. Lew \\ Structures Division \\ 301-975-6061
}

Ann Bieniawski

Structures Division

301-975-6065

\section{Sponsor}

Federal Emergency Management Agency Mitigation Directorate

\section{Objective}

BFRL will provide technical review of FEMAfunded projects to develop improved building codes for seismic design of new construction.

\section{Problem}

FEMA supplements the capabilities of its staff by using the BFRL's technical expertise.

\section{Approach}

During FY 1996, BFRL will be a member of mutually agreed upon technical subcommittees of the BSSC ongoing update of NEHRP Recommended Provisions for the Development of Seismic Regulations for New Buildings. BFRL will review all ballot submittals from the Provisions Update Committee. At the request of the FEMA Project Officer, BFRL staff will participate in proposal evaluation panels and selected technical seminars and meetings.

\section{Recent Results}

No published results. 


\section{Secretariat U.S.-Side Panel on Wind and Seismic Effects}

\author{
Principal Investigator \\ Noel J. Raufaste \\ BFRL Headquarters \\ 301-975-5905
}

\section{Sponsor}

National Institute of Standards and Technology and

10 Federal Agency Members

\section{Objective}

To provide the U.S.-side Secretariat of the U.S.Japan Panel on Wind and Seismic Effects.

\section{Problem}

Loss of life and property result from insufficient knowledge to design adequately and construct building and other structures cost-effectively against high winds and seismic loads. Improved mitigation practices are in early stages of development. Technical collaborations between experts in the United States and Japan continue to produce findings that improve natural hazard mitigation practices. Such improvements are possible through joint working relations with experts from the United States and Japan through performing joint research projects and exchanges of research personnel, technical data and information, and research equipment. This Panel is part of the U.S.-Japan Natural Resources Development Program operating under the aegis of the U.S.- Japan Cooperative Science Program of 1961.

\section{Approach}

During FY 1996, BFRL will:

1. Plan U.S.-side activities (18 Agency membership) through hosting the panel's Annual Joint Meeting and managing the two U.S. Panel's domestic meetings.

2. Plan and coordinate technical activities of the 11 Task Committees.

3. Manage U.S. delegation to Japan-side hosted meetings and workshops and developing technical programs for U.S. hosted meetings.

4. Develop a draft Panel Home Page

5. Maintain liaison with U.S. and Japan Panel members and other experts associated with the Panel's activities.

6. Plan Japan Panel member visits to the United States under the auspices of the Panel, e.g., post disaster investigations, special studies, data gathering.

7. Maintain administrative and technical records.

8. Prepare and distribute periodic activities reports, and other materials as appropriate to U.S.-side Panel and Task Committee members.

9. Participate in Joint Panel meetings and Task Committees, and

10.Prepare and publish annual Proceedings.

\section{Recent Results}

Proceedings of the 27th Joint Panel Meeting on Wind and Seismic Effects, Technical Memorandum No. 3387, Public Works Research Institute, Japan, May 1995.

Proceedings of the 6th U.S.Japan Workshop on Earthquake Disaster Prevention for Lifeline Systems, Public Works Research Institute, Japan, July 1995.

Proceedings of the International Workshop on Wind and Earthquake Engineering for Offshore and Coastal Facilities, January 17-19, 1995, Public Works Research Institute, Japan, 1995.

Proceedings of the 10th U.S. Japan Bridge Engineering Workshop May 10-11, 1994, Federal Highway Administration, 1994.

Proceedings of Workshop on Strong Motion Data, Vol 1 and 2, December 13-17, 1993, Port and Harbour Research Institute, Japan, 1994.

Proceedings of the Fourth U.S.-Japan Workshop on Soil Liquification, July 4-6, 1994, Public Works Research Institute, Japan, July, 1994.

Proceedings of the UINR Tsunami Workshop, Public Works Research Institute, Japan, September, 1994. 



\section{STRUCTURES DIVISION}

STRUCTURAL ENGINEERING 



\section{Construction Automation}

Principal Investigators

William C. Stone

Structures Division

301-975-6076

Lawrence E. Pfeffer

Structures Division

301-975-6067

\section{Sponsor}

National Institute of Standards and Technology

\section{Objective}

To develop new techniques and standards for real-time construction metrology and to develop a national standard for kinematic modeling of construction site machinery and displaying robust graphical representations which depict the real time state of the machine to a remote node on the information superhighway.

\section{Problem}

The emergence of high speed computer communication networks (the "information superhighway") and the rapid advance of realtime, immersive, computer graphics (virtual reality) technologies presage the imminent ability to manage remote construction machines; and to provide information on the state of such machines to operators (on-site or remote) that would greatly enhance their productivity. Limited demonstrations have been performed on this type of technology, largely relating to the control of robotic spacecraft and, more pertinently, to teleoperation of simple machines for handling nuclear waste.

To achieve acceptance in the construction industry, a standard means of rapidly interfacing any piece of machinery to a construction-site database must be developed. The underpinning to the above technology is the ability to know the real-time position of any piece of equipment and component on the construction site. Present surveying tools suffer from many shortcomings, the most important is they must operate under line-of-sight (LOS) conditions. The development of a non-line-of- sight surveying system, which can in effect "see through walls," represents the "holy grail" of constructions metrology.

\section{Approach}

During FY 1996, BFRL will perform two tasks:

1. Construction Automation Test Bed.

Research will focus on the processing and display of the real time data returned from the construction site, relating to the global position and attitude ( 6 degrees of freedom) that define the spatial state of a construction machine on the surface of the earth, and appropriate sensor data which provide information on the state of all other local degrees of freedom (e.g., the angular rotation of each joint of a backhoe.) The objective is to allow construction company management to ascertain, in a visually realistic manner, the status of the machine and all its key components in real time from any location on earth.

Where the proposed work departs from traditional modeling schemes (e.g., Bechtel's "Walk Through") BFRL's work animates the scene. BFRL will realistically model the change in position of various objects as updated data is received from telemetry uplink. This work will demonstrate (using the BFRL's tri-directional test facility as the initial construction site test bed) the ability to capture position, orientation, and action and transmit this information to a "remote" immersion graphics system where an observer can observe simple actions via computed tele-presence.

Work will commence on the development of vehicle representation standards and software tools to convert existing CAD models to seamlessly interface with the proposed standards. The specific technical approaches in FY 1996 on the establishment of a Virtual Construction Test bed are as follows:

- Determine key issues concerning the functionality, interface, and implementation of a database to serve as a "world model" for displays/simulation/automation of construction sites. Develop a short-term, 
limited function surrogate for the database to facilitate initial progress on the virtual construction site simulator. Investigate how functionality/interface aspects will influence future constructionsite database development. Determine best strategies to facilitate ease of use with structural data typical of the construction industry (e.g., existing open standards $\{$, e.g., STEP $\}$ or modifications of it.)

- Determine a prototype virtual construction site simulator/monitor, using 3-dimensional graphics to display/simulate a construction site. This system will display a high-fidelity graphical model of a construction site (or suitable surrogate), with the position, orientation, and configuration of the site's movable objects based on a database-derived kinematic model or, optionally (if available), telemetered sensor data.

- Investigate the currently-available systems for 3-D relative position sensing over medium and larger workspaces; current candidates are magnetic, ultrasonic, optical, and GPS-based systems. Investigate the use of Global Positioning System technology for determining the absolute position and orientation of parts at construction sites. Determine the performance of representative, currently-available GPS equipment. Determine what technical approaches/modifications are most promising to increase differential accuracy, bandwidth, and robustness to interference. Gather information to support cost estimates for developing improved GPS-based sensing systems. Determine the feasibility -- and most promising approaches -- for merging GPS data with alternate metrology techniques to create a consistent kinematic model of the site of interest.

- Investigate wireless communication techniques to support the Construction Automation Project's needs for wireless telemetry and node-to-node communication; specifically address bandwidth, interface standards, cost, and
FCC licensing issues. Determine needs for subsequent research and development.

2. Non-Line-of-Sight Metrology. BFRL will expand its work that proved the viability of NLS technology to effectively "survey through solid concrete." The results of this research will provide the ability to track vehicles, construction components, and personnel inside a building, no matter what the state of construction. It would permit the use of a registered-view helmet mounted display systems for placement of construction components, or for guiding repair and retrofit operations. It would extend to far different fields of endeavor, including the tracking of firefighters inside a smoke filled building. Work will concentrate on defining propagation and error characteristics of spread spectrum radar as a function of material property and class (e.g., glass, concrete, wood etc.), frequency bandwidth, power, signal-to-noise ratio (and techniques, both physical and analytical for improving the same), and obstacle geometry (including thickness, distance from transmitter to object, and distance from receiver to object). These data will form the basis for follow-on work which will permit investigation of auto-compensation systems which will account for propagation delays.

\section{Recent Results}

Stone, William, C., Ed., NIST Construction Automation Program Report No. 2: Proceedings of the NIST Construction Automation Workshop, March 30-31, 1995, NISTIR 5856, National Institute of Standards and Technology, May 1996.

Stone, William, C., NIST Construction Automation Program Report No. 1 Non-Line-ofSight (NLS) Construction Metrology, NISTIR 5825, National Institute of Standards and Technology, February 1996. 


\section{High-Performance Concrete: Design and Construction Practices}

\author{
Principal Investigators \\ Nicholas J. Carino \\ Structures Division \\ 301-975-6063
}

Dat Duthinh

Structures Division

301-975-4357

\section{Sponsor}

National Institute of Standards and Technology

\section{Objective}

To enhance the application of high-performance concrete in the construction of new facilities and the rehabilitation of existing ones.

\section{Problem}

High-strength concrete offers the potential for cost savings in construction due to reduced member dimensions, the capability to accommodate rapid construction schedules, and enhanced service life. However, from a structural perspective, high-strength concrete is inherently more brittle than normal strength concrete. Therefore, failure mechanisms in structural members need to be understood before appropriate design criteria can be developed. Lacking the necessary knowledge, design codes have taken a conservative approach and have limited the concrete strength that can be used to compute member resistance to tensile failure modes.

Testing is essential to assure that the concrete delivered to a construction site has the potential to develop the desired properties. Also, in-place testing is essential to assure that the finished product has the specified properties. Standards for acceptance testing have been developed based on experiences with normal strength concrete. Problems have been encountered when current standards are used to evaluate high-strength concrete specimens. Laboratories testing the same material have obtained divergent strength values, which has resulted in unnecessary disputes. Testing standards need to be updated to extend their applicability to high- strength concrete. Developing tools that can be used in the field to evaluate test results in a rigorous fashion can promote the use of in-place tests to determine conformance to performance requirements.

Current prescriptive curing requirements which are more than 50 years old, may not be applicable to high-performance concrete mixtures. In addition, current standards make no distinction between curing to obtain adequate strength and curing to obtain adequate durability.

The benefits of high-performance concrete are given in CERF Report \#94-5011 High-

Performance Construction Materials and Systems: An Essential Program for America and Its Infrastructure. This report, developed by a cooperative effort of key individuals from industry, academe, and the Federal government, identifies research related to high-performance concrete to enhance the competitiveness of the U.S. construction industry and reduce the life-cycle costs of constructed facilities. It notes impediments are preventing exploitation of the unique properties and advantages of high-performance concrete.

\section{Approach}

During FY 1996, BFRL will recognize improvement of the ACI-318 criteria for shear strength as the first research area related to structural performance of high-strength concrete. A state-of-the art report will review past research, discuss alternative design models, and propose an experimental research program. This effort will include a parametric study of the compression field theory and physical tests. Physical tests will provide the fundamental information on the post-cracking behavior of high-strength concrete needed to implement the compression field theory.

A grant was awarded to Cornell University to perform a comprehensive review of ACI 318 Standard to identify other design provisions whose applicability to high-strength concrete is uncertain. The findings will provide a comprehensive research plan on the structural performance of high-strength concrete. Work will address test specimen preparation and the applicability of unbonded caps. Results will be 
synthesized and developed into recommendations for ASTM Committee C9. Work will address test specimen preparation and the applicability of unbonded caps. Results will be synthesized and developed into recommendations for ASTM Committee C9. Research continues to develop the basis for updating current ASTM test methods to extend their applicability to high-strength concrete.

In-place strength tests will be key elements of performance-based specifications. An impediment to their widespread use is the lack of convenient tools that can be used in the field to analyze test results. A contract was awarded to Purdue University to develop a user-friendly computer system to analyze in-place test results. The system carries out the statistical procedures previously developed at BFRL. The system will be presented to ACI Committee 228 for consideration as a standard practice.

A state-of-the-art report on curing highperformance concrete is being prepared. The report will include a research plan to provide the technical basis for recommended curing practices (or an expert system) for highperformance concretes, that will ensure the required strength and durability. It is expected that modeling water diffusion through maturing concrete will be an important aspect of the research. Working with BFRL's Building Materials Division, work will center on developing a reliable test method to assess the effects of different curing conditions.

Subsequent experiments will investigate the effects of curing conditions on the strength and transport properties. Results will be synthesized into a suitable form for presentation to ACI Committees 308, 318 , and 363 for incorporation into $\mathrm{ACI}$ reports and standards.

\section{Recent Results}

Carino, N.J., Effects of Testing Variables on the Measured Compressive Strength of High-Strength (90 MPa) Concrete, NISTIR 5405, National Institute of Standards and Technology, October 1994.

Carino, N.J., Guthrie, W.F., Lagergren, E.S., and Mullings, G.M., "Effects of Testing
Variables on the Strength of High-Strength (90 $\mathrm{MPa}$ ) Concrete Cylinders," Proceedings of the $\mathrm{ACI}$ International Conference on High-Performance Concrete, ACI SP-149, pp. 589-632, November 1994. 
Fiber Reinforced Polymer Composite Structures in Construction

\author{
Principal Investigator \\ Dat Duthinh \\ Structures Division \\ 301-975-4357
}

\section{Sponsor}

National Institute of Standards and Technology

\section{Objective}

To characterize the structures built of and/or strengthened/repaired with Fiber Reinforced Polymer (FRP) composite materials and to develop the technical basis for efficient use of fiber-reinforced polymer composites in construction.

\section{Problem}

The construction industry is in need of advanced, high-performance materials to reduce overall, life-cycle costs of constructed facilities. While traditional construction materials have served well, economic pressures in local and global construction markets have created a critical need for higher performance materials. The families of modern FRP composites are advanced and versatile materials which have many potential applications in the construction industry. When compared with traditional materials, composite materials have many advantages including reduced weight and higher static and fatigue strength, resistance to chemicals and corrosion, electromagnetic transparency, part/member consolidation, low tooling and finishing costs, wide versatility in structural form, and low overall life-cycle costs. While FRP composites are being used (or considered for use) nationwide in a variety of demonstration projects focusing on bridges, bridge retrofit, concrete reinforcement, marine infrastructure, and seismic building upgrades, several constraints impede widespread commercialization. One constraint is the lack of adequate design codes and an incomplete technical basis from which to develop design methods.

\section{Approach}

During FY 1996, BFRL will conduct a review of the literature to determine research needs and directions. Interaction with staff in BFRL's Building Materials and Building Environment Divisions will be pursued. BFRL will contribute to the development of consensus performance criteria and design methodology for structures built of and/or strengthened/repaired with FRP composites.

\section{Recent Results}

New project 


\section{High-Performance Steel}

\author{
Principal Investigator \\ John L. Gross \\ Structures Division \\ 301-975-6068
}

\section{Sponsor}

National Institute of Standards and Technology

\section{Objective}

To develop the technical basis for new structural components, connections and systems that can exploit the advantages of High-Performance Steel (HPS) and overcome limitations, and to develop design methods and specifications for the design of these HPS systems.

\section{Problem}

Design specifications for fabricated steel buildings and bridges and the hot rolled structural shapes in use today were developed when the dominant steel for construction had a yield strength of $230 \mathrm{MPa}$. Owing to refinements in steel-making processes such as micro alloying and innovative production processes, yield strengths in excess of $100 \mathrm{ksi}$ $(690 \mathrm{MPa})$ may be achieved while favorable yield-to-ultimate strength ratios and weldability are maintained.

Current specifications are based explicitly on material yield strength and ultimate strength. However, these metrics are not sufficient to characterize the complete stress-strain behavior of all steels, and in particular, high-strength HPS. (The older steels have mechanical postyield properties that are significantly different those of HPS such as strain hardening behavior and yield-to-ultimate strength ratio). To remove this barrier, specifications are required for the design of steel buildings and bridges which are based on the complete characterization of the steel to be employed thus making design "open" to any constructional steel which either is, or becomes, available. Because such an approach would address not just the yield and ultimate strengths but rather the complete stress-strain behavior (yield-to-ultimate ratio, ductility, etc.) it would provide a consistent level of reliability among the various steel choices; a condition which is not achieved with current design specifications.

This research specifically addresses priority research on "Innovative Structural Shapes" under the "Products and Systems" research topic and "Advanced Analysis Methods" under the "Analysis and Design" research topics as identified in Materials for Tomorrow's Infrastructure: A Ten-Year Plan for Deploying HighPerformance Construction Materials and Systems, Civil Engineering Research Foundation.

\section{Approach}

During FY 1996, BFRL will perform five tasks:

1. Ascertain the applicability of current design specifications to HPS.

2. Identify and quantify the characteristics of currently available steel sections that contribute to premature failure (e.g., local instability).

3. Study analytically the performance of sections optimized for superior performance with HPS.

4. Verify experimentally the performance of sections fabricated/rolled from HPS and ability to analytical predict the performance.

5. Develop the technical basis for highperformance steel design and construction standards.

The application of HPS will be driven by its high strength, toughness and weldability but potential obstacles must be overcome to use HPS fully. Local and/or overall instability may prevent a lighter and more slender HPS member from achieving its yield strength or may significantly reduce its inelastic rotation capacity after yielding. New cross-section shapes optimized for superior performance with HPS steels are required.

\section{Recent Results}

New project 


\section{Fire Resistance of High-Strength Concrete}

Principal Investigators

L.T. Phan

Structures Division

301-975-6077

Dat Duthinh

Structures Division

301-975-4357

\section{Sponsor}

National Institute of Standards and Technology

\section{Objective}

To gain an understanding of the performance of high-strength concrete (HSC) when exposed to high temperatures as would occur during fires.

\section{Problem}

HSC is gaining in use in structural applications, such as columns of high-rise buildings, in prestressed concrete members, and in offshore structures. However, little is known about the fundamental behavior of HSC when exposed to a fire. A few tests have indicated an alarming tendency for explosive spalding of structural members under fire conditions. The current belief is that the poor performance is due to the extremely low porosity of the cement paste that prevents the escape of steam, leading to high internal pressures that spall the concrete. To assure life safety, understanding the fire endurance of HSC is essential. This research topic is listed in CERF Report 94-50 11, which outlines the 10-year plan for research on highperformance construction materials and systems (HPCMS).

\section{Approach}

During FY 1996, BFRL will prepare a state-ofthe-art report to document past and ongoing studies on the fire resistance of HSC. The report will include a review of the various damage mechanisms, including the role of moisture transport, when concrete is exposed to fire. In addition, techniques developed to model and predict fire damage to concrete will be reviewed. If suitable modeling tools are available, they will be used for initial parametric studies to gain insight into the factors that control performance during fire exposure. Such insight will be useful to develop a comprehensive long-term research program to understand the damage mechanism(s) when HSC is exposed to fire. Since this is a multidisciplinary project, the PI's will interact with appropriate experts in BFRL's Building Materials, Building Environment, and Fire Safety and Engineering Divisions to formulate the research program. It is envisioned that, once a test-calibrated model of the fire resistance of HSC is formulated, such model can be implemented as a module of a computer program to assess the global resistance of an HSC structure to fire. One computer program which might serve as the platform for the model is the program SAFIR, currently under development at the University of Liege, Belgium.

Following the completion of the state-of-the-art report, an experimental program will be planned. The objectives of this test program are to: 1. obtain first hand a basic understanding of the performance of HSC when subjected to elevated temperature at a high rate; and 2. validate (or refute) observations and findings in other studies. This test program will be planned and conducted in collaboration with BFRL's Fire Safety Engineering Division. The findings from this initial test program will be used, with information obtained from the state-of-the-art report, in developing more comprehensive, future research plans for this project.

\section{Recent Results}

New project 


\section{Next Generation Design Standard for Wind Loads}

\author{
Principal Investigator \\ Emil Simiu \\ Structures Division \\ 301-975-6076
}

\section{Sponsor}

National Institute of Standards and Technology

\section{Objective}

To develop key elements of an improved wind load design standard that will use existing databases and state-of-the-art computational methods for establishing site-specific design wind speeds and the corresponding steady and fluctuating structural loads.

\section{Problem}

There are many significant deficiencies in the wind load design provisions of current U.S. codes and standards. They include: 1 . extreme wind speeds and corresponding load factors derived from outmoded estimation methods; 2. exposure coefficients that cannot deal with complex wind exposures (the normal case); 3. gross simplifications of extreme pressures and loads that ignore the directional effects of wind; and 4. no distinction between design for safety and design for serviceability.

Besides the reliable estimation of design loads, there is an urgent need for improved methods by which to assess wind speeds, and thus the wind forces, in complex wind exposures following extreme events such as hurricanes. Only then is it possible to evaluate with reasonable confidence the performance of structures subjected to wind loads approaching or exceeding the design limit states.

\section{Approach}

During FY 1996, BFRL will:

1. Provide the application of the "peaks over threshold" extreme value estimation approach to hurricane winds and

2. Develop a framework for interactive standard provisions using climatological and aerodynamical databases.

This work will combine directional extremes for a given site with wind tunnel databases for force and pressure coefficients that most closely matches the geometry and/or dynamic characteristics of the structure. Of major interest is the tail length of distributions that best fit a given set of annual extreme wind speeds. The widely used Gumbel distribution has an infinite tail length while physical grounds require that wind speeds be bounded. Recently, statistical methods for estimating those bounds have become available. In complex wind exposures, analytical models based on fundamental principles of fluid mechanics and on certain empirical relationships offer a substantial improvement over current equilibrium boundary layer models on which site-specific wind speeds are based. Since force and pressure coefficients are direction-dependent, directional distributions of extreme wind speeds can be handled most conveniently in combination with these coefficients. Finally, the development of reliability concepts will make it possible to match safety and serviceability criteria with given levels of risk under wind loading.

\section{Recent Results}

Simiu, E and Stathopoulos, T., "Codification of Wind Loads on Buildings Using Bluff Body Aerodynamics and Climatological Data Bases," Proceedings, 3rd International Colloquium on Bluff Body Aerodynamics and Application, Virginia Polytechnic Institute and State University, July 1996.

Simiu, E., and Heckert, N.A., Extreme Wind distribution Tails: 'Peaks Over Threshold' Approach, NIST Building Science Series 174, National Institute of Standards and Technology, March 1995 and Journal of Structural Engineering, Vol.122, pp539-547, May 1996.

Whalen, T.M., Probabilistic Estimates of Design Load Factors for Wind-Sensitive Structures Using the 'Peaks Over Threshold' Approach, Technical Note 1418, National Institute of Standards and Technology, April 1996.

Simiu. E., Heckert, N.A., and Whalen, T.M., Estimates of Hurricane Wind Speeds by the 'Peaks Over Threshold' Method, Technical Note 1416, National Institute of Standards and 
Technology, February 1996.

Schechter, M.M., Schechter, E., and Simiu, E., Developmental Computer Version of ASCE 7.95 Standard Provisions for Wind Loads, NIST

Technical Note 1415, National Institute of Standards and Technology, November 1995.

\section{Assessment of Tubular Steel Joints in Existing Offshore Structures}

Principal Investigator

Andrew Taylor

Structures Division

301-975-6078

\section{Sponsor}

Department of Interior

Minerals Management Scrvice

Technology Assessment and Research Branch

\section{Objective}

To conduct a workshop for assessing the stateof-the-art and future research needs related to tubular steel joints in offshore structures.

\section{Problem}

About 3800 offshore platforms are operating in the Gulf of Mexico. Many of these are in shallow water, and constructed more than 40 years ago. Most of these platforms are still productive. However, the code-specified design level wave heights have increased dramatically since the time when the platforms were designed and constructed. Consequently, these older platforms are significantly under-designed by today's standards. More recently, several major platforms have been constructed off the West Coast of the United States. These platforms are in zones of high seismicity, and may be subjected to severe earthquake loads. Very little is known about the cyclic inelastic behavior of joints in offshore platforms subjected to either extreme storms or to earthquakes.

When under-designed platforms are exposed to extreme loadings during a storm or earthquake, they can undergo several cycles of inelastic deformation. These deformations may not be large enough to cause immediate failure of a joint or frame, but the cumulative effect of plastic deformations caused by a series of moderate storms or earthquakes over many years may eventually lead to failure. What is the remaining service life of these structures which experienced one or more damaging storms or earthquakes? 
Recently implemented Federal regulations have prompted reexamination of many existing off shore platforms. Under Minerals

Management Service (MMS) policies, of fshore platforms must periodically be re-certified as to their safety and capacity. Petroleum production companies increasingly have found it difficult to re-certify older platforms because there is a lack of laboratory test data and analytical methods available to verify platform capacity under inelastic cyclic loading. As a result, older platforms must sometimes be decommissioned and removed - an extremely costly procedure.

\section{Approach}

During FY 1996, BFRL will prepare a summary report containing the papers presented at its workshop, Houston during 5-6 September 1995, to define the state-of-the-art, and to identify research needs, for tubular steel joints in offshore structures subject to high amplitude cyclic loading. The workshop focused on issues relating to requalification of existing offshore structures. Approximately 30 representatives of the petroleum industry, research institutes and universities attended. The summary of the report will contain the discussions and conclusions reached at the workshop.

\section{Recent Results}

New project 


\section{BUILDING MATERIALS}

DIVISION

\section{COATINGS AND OTHER MATERIALS}



Coatings Service Life Prediction Consortium

\author{
Principal Investigator \\ Jonathan Martin \\ Building Materials Division \\ 301- 975-6717
}

\section{Sponsor}

National Institute of Standards and Technology

\section{Objective}

To demonstrate and validate methods that mathematically predict and relate the photodegradation response of coating systems exposed to UV-radiation in the laboratory and field, for quantitatively characterizing the photodegradation response, and modeling temporal changes in solar spectral radiation.

\section{Problem}

UV-radiation, wetness and temperature of a material, are considered the prime weathering factors in determining the service life of coating systems exposed outdoors in most nonindustrial environments. The inability to control or adequately characterize temporal changes in these weathering factors is a major challenge to overcome before establishing a connection between the degradation response of polymeric materials exposed in the laboratory and the field. Needed is the correlation between degradation effects of UV-radiation in the field and in the laboratory.

\section{Approach}

During FY 1996, BFRL will quantify photodegradation effects of uv-radiation in the field and laboratory. The work will be performed at a consortium member laboratory. A model was identified and further refined for mathematically relating photodegradation results obtained from field and laboratory exposure experiments. This model requires knowledge of a material's spectral UVabsorbency and its spectral quantum yield along with knowledge of the spectral UV-irradiance to which it is exposed.

This research will be extended to include all photolytically active wavelengths such as modeling spectral UV-radiation -- time series and other mathematical models for modeling temporal changes in the solar spectral UVradiation and determining the number of spectral wavelengths to model the photolytically active wavelengths between $290 \mathrm{~nm}$ and 325 $\mathrm{nm}$. Photolytically active wavelengths will be determined from spectral quantum yield experiments performed in the laboratory. Key questions will include the range of wavelengths over which quantum yield response is positive, the number of wavelengths required fully to specify the absorption and quantum yield spectra, and the effect of experimental error of filters having different half-widths. The validity of the proposed model will be learned by exposing the same coatings in the field and laboratory and comparing the results. In this experiment, the field exposure sites will be equipped with a spectral filter radiometer while the spectral quantum yields and absorption spectra will be determined in the laboratory.

\section{Recent Results}

Floyd, F.L. and Martin, J.W., "Influence of Reliability Theory on R\&D Planning and Management of Risk," Farbe und Lack, V.102, p28, 1996.

Frohnsdorff, G.J. and Martin, J.W., "Toward Prediction of Building Service Life-The Standards Imperative," Proceedings of the 7th International Conference on the Durability of Building Materials and Components, Stockholm, Sweden, May 1996.

Martin, J.W., Saunders, S.C., Floyd, F.L., and Wineburg, J., "Methodologies for Predicting Service Lives of Coating Systems," Federation of Societies of Coatings Technology Monograph Series, 1996.

Nguyen, T. and Martin, J.W., "Modes and Mechanisms of Degradation of Epoxy-coated Reinforcing Steel in a Marine Environment," Proceedings of the 7th International Conference on the Durability of Building Materials and Components, Stockholm, Sweden, May 1996. 
Integrated Knowledge System for Corrosion-Protective Coatings for High-Performance Steel

\author{
Principal Investigators \\ Lawrence Kaetzel \\ Building Materials Division \\ 301-975-5912 \\ Mary McKnight \\ Building Materials Division \\ 301-975-6714
}

\section{Sponsor}

National Institute of Standards and Technology

\section{Objective}

To develop an architecture, standard knowledge formats, information model, and operational computerized systems for representing and disseminating organic coatings knowledge for protecting high-performance steel structures against corrosion.

\section{Problem}

Coatings provide a versatile, cost-effective means for protecting steel structures against adverse environmental conditions, but decisions about coating system selection are of ten difficult to make. Currently, users of coating systems must rely on information represented in many different forms and systems. These include manuals, guides, photographs and drawings, videos, databases, and other references. In addition, a human expert's advice may be necessary to reach a decision. Often, the decision-making knowledge may be dispersed or unavailable. Currently, no comprehensive systems define these entities and address high performance construction materials. An integrated knowledge system that includes different forms of knowledge and is presented in a coherent format will improve the specification, selection, and use of coatings systems for protecting steel, thus improving the performance and extending the service life of structures.

\section{Approach}

During FY 1996, BFRL will 1. distribute its Coatings Expert Advisory (COEX-I) expert system to coatings industry users, 2 . develop database formats for representing the properties of coatings formulation and performance characterization, and 3 . investigate and test the application of intelligent agent technologies for enhancing the exchange of coatings knowledge between industry and government organizations. BFRL will develop an improved decision-making process and a repository of knowledge for use by the construction industry that help extend the service life of these structures. The work in developing an Integrated Knowledge Based System include defining and establishing an architecture, information model, and developing computerized prototypes and operational systems. Initially, the domain and audience will be narrowly focused, then expanded to address more comprehensive (deep knowledge) systems for multi-disciplines (e.g., material suppliers, designers). For example, COEX-I contains knowledge on the failure, remedial action, and selection of coatings systems for stationary, military structures (including steel substrates). The system integrates multiple forms of knowledge, including high-level expert reasoning, audio/video, databases, photographs, and bibliographic references. Eventually, this system could be expanded to address additional structure types (e.g., bridge management systems for highway structures).

The COEX-I expert system developed in 1995 will be distributed to coatings industry users for their review and response during 1996. In 1996, two new methods will be evaluated, and tested for their application to the coatings knowledge domain: 1. development of a database format to represent properties of coatings materials and data sets from laboratory and field tests on performance, and 2. automated techniques for the exchange of knowledge between humans and computers.

BFRL has developed a draft database format that will be submitted to the Steel Structures Painting Council for refinement and possible adoption as a standard. Since databases are a valuable form of coatings knowledge, this activity should promote a greater exchange of knowledge and provide a basis for computercomputer knowledge transfer. An example of 
their importance, is demonstrated by the need to determine the effects of solar UV-B radiation on coatings performance where a database is being developed under a separate project from the NIST Standard Reference Data Program (SRDP).

New methods for improved dissemination and access to knowledge bases will be investigated, including the development of intelligent agents that allow more efficient computer-computer and human-computer interactions. A testbed (computerized system and software development tools) will be established at BFRL that will provide a platform for developing and testing intelligent agents, and database servers. The testbed will be accessible through highspeed data links to other organizations including the University of Kansas, NIST's Physics Laboratory, Steel Structures Painting Council, and the Infrastructure Technology Institute at Northwestern University. Standards activities such as STEP, Electronic Data Exchange (EDI), Knowledge Query Language (KQML) will be evaluated and implemented if they apply.

\section{Recent Results}

Developed an information model and architecture for coating materials databases and installed on an Internet World Wide Web site. Its address is http://ciks.cbt.nist.gov

Established an SSPC Working Group -. Committee C.4.10 on Knowledge Based Systems for Coatings.

\section{Database Architectures for the Properties of Construction Materials}

\author{
Principal Investigators \\ Lawrence J. Kaetzel \\ Building Materials Division \\ 301-975-5912
}

Mary E. McKnight

Building Materials Division

301-975-6714

\section{Sponsor}

National Institute of Standards and Technology

\section{Objective}

To demonstrate the use of a database architecture for representing and exchanging material properties data for coatings and cementitious materials.

\section{Problem}

There is a lack of consistency in the formats and procedures used to represent and exchange materials properties database information among construction industry users. This results in difficulties when attempting to characterize the performance of materials and comparing the results from laboratory and field tests, or simply in the exchange of information electronically. The problems are manifested by the fact that database development efforts are: 1) biased by the originator, 2) software and hardware platforms are inconsistent, and 3) there is an absence of the use of information standards. These situations result in a less-than-optimal level of decision-making, due in part, to the absence of relevant data.

\section{Approach}

During FY 1996, BFRL will establish, test, and demonstrate databases using the following methods and activities:

1. develop standard formats for representing the properties of coating materials and cementitious materials,

2. apply the ISO Standard 9579, Remote Data Access (RDA), generic model to construction industry information needs, and

3. develop and implement database information using the ANSI Standard 
X3.135.992, SQL Relational Database Model.

The tasks will rely on using BFRL's National Computer-Integrated Knowledge System Network (CIKS) test bed for implementing and testing data sets against the architecture. The Internet World Wide Web framework will be used as the point of access to the database. Success in developing a model architecture will be determined, largely by the application of many test data sets of various types and sources. Currently, the test results from the AASHTO Materials Reference Laboratory (AMRL), Paint Proficiency Program and the ASTM Cement and Concrete Reference Laboratory (CCRL) are being evaluated for implementation. Tools will be developed so that the feasibility and usefulness of the database formats can then be judged by a user base from industry and government, through access provided by the NIST CIKS test bed. This will provide the basis for a non-proprietary architecture that can be implemented within the construction industry materials user communities, and one that is based on information standards. Examples of additional data sets include material product data sheets and safety data sheets on coating materials. These will be provided by coating manufacturers and suppliers.

\section{Recent Results}

New project

\section{Performance Criteria for Enclosures for Lead-Based Paint}

\author{
Principal Investigators \\ Walter Rossiter \\ Building Materials Division \\ $301-975-6719$
}

Mary McKnight

Building Materials Division

301-975-67l4

Sponsor

U.S. Department of Housing and Urban

Development

Office of Lead-Based Paint Abatement and

Prevention Poisoning

\section{Objective}

To develop preliminary performance criteria for mechanically attached enclosures for lead-based paint on interior and exterior walls of housing.

\section{Problem}

The U.S. Department of Housing and Urban Development (HUD) concluded from the results of a demonstration project that liquidapplied encapsulant may be less expensive in abating lead-based paint hazards than mechanically attached enclosures. Situations may arise, however, where enclosures are more appropriate than encapsulation. Presently, no suitable, generally accepted performance criteria exist for the selection or assessment of enclosure materials. HUD has requested BFRL to prepare the needed criteria on an emergency basis.

\section{Approach}

During FY 1996, BFRL will identify performance requirements and test procedures for mechanically attached enclosure materials applied to interior and exterior walls.

Preliminary performance criteria will be developed based primarily on current knowledge and secondarily on short-term tests.

This criteria will address enclosure characteristics that are effective in keeping the lead in place and to keep contamination of the environment at a very low risk for the desired time. Additionally, the criteria will address 
safety characteristics such as resistance to fire as defined by codes or other regulations.

Research will focus on the mechanical and physical properties of enclosure materials and components. Included are enclosure characteristics that prevent the release of lead-contaminated particles (e.g., structural integrity, abrasion resistance, and impact resistance), and characteristics associated with health and safety (e.g., fire resistance). BFRL will:

1. Identify performance attributes and requirements for enclosure materials and systems,

2. Review literature to develop a current knowledge database,

3. Develop the preliminary performance criteria based on current knowledge,

4. Identify gaps in the knowledge base,

5. Conduct tests, as necessary, to fill the gaps in the knowledge base, and

6. Revise the preliminary performance criteria based on the test results.

ASTM standards will be used where applicable as testing is done. The tests will address mechanical and physical properties associated with the effectiveness of the enclosure materials and systems in forming a barrier against the escape of lead-contaminated particles. BFRL also will address the efficiency of the enclosure to prevent the release of lead-contaminated particles through gaps and cracks in the mechanically attached enclosure due to air movement.

During the study, sites will be visited where leaded-paint surfaces have been enclosed or where the installation of enclosures is being considered. The visits will be to assess properties of surfaces being considered for encapsulation and to gain first-hand knowledge about the suitability of commonly used enclosure materials and systems.

Results will be provided to ASTM E06.23 to use in the development of standards for enclosure materials and systems.

\section{Recent Results}

New project
Performance Criteria for Selection of Liquid-Coating Encapsulant for Lead-Based Paint

\author{
Principal Investigators \\ Walter Rossiter \\ Building Materials Division \\ 301-975-6719 \\ Mary McKnight \\ Building Materials Division \\ 301-975-6714
}

\section{Sponsor}

U.S. Department of Housing and Urban

Development

Office of Lead-Based Paint Abatement and

Poisoning Prevention

\section{Objectives}

To develop preliminary performance criteria for liquid-coating encapsulant for lead-based paint.

\section{Problem}

In a survey of the nation's housing, the U.S. Department of Housing and Urban Development (HUD) found that about 57 million homes in the United States have lead-based paint (LBP) on at least one painted surface. One way of helping to ensure that the hazards of LBP in housing are kept low is to protect the paint by a liquid-coating encapsulant. A major deterrent to the use of these encapsulant is the lack of performance standards and field performance data.

\section{Approach}

During FY 1996, BFRL will develop preliminary performance criteria based on early age or shortterm laboratory and field tests. This work involves identifying performance requirements and test procedures. On the most general performance level, encapsulant must, with an acceptable probability, ensure that the encapsulant keeps the lead in place and prevents it from contaminating the environment for the desired time. The performance criteria must address encapsulant material characteristics that prevent release of lead such as abrasion resistance, impact resistance, leaching resistance and ability to 
prevent failure (e.g., peeling, flaking and chipping) of the underlying LBP layer.

BFRL will:

1. Visit several field sites where encapsulant have been installed or are being considered for use to identify specific requirements and select test materials.

2. Select substrate test materials and develop procedures for preparing them in a uniform and consistent manner to simulate conditions expected in the field.

3. Select encapsulant and control paints to use in testing.

4. Select appropriate test procedures using ASTM tests where appropriate and prepare test specimens.

5. Subject specimens to laboratory performance tests under conditions in which the applied stresses are at the high ends of the ranges expected under service conditions.

6. Based upon the results of the laboratory tests, carry out limited field testing. Using both data sets, preliminary performance criteria will be developed.

Results of the research will be provided to ASTM Committee E06.23, Abatement of hazards from Lead in Buildings, to use in improving the technical bases for standards for encapsulant. Following this work, BFRL will address long-term laboratory aging and weathering tests and additional field tests to provide data for verifying and improving the preliminary performance criteria.

\section{Recent Results}

New project

\section{Method for Evaluation of Performance of Lead-in-Paint Measuring Devices Under Simulated Field Conditions}

\author{
Principal Investigator \\ Mary McKnight \\ Building Materials Division \\ $301-975-6714$
}

\section{Sponsor}

U.S. Department of Iousing and Urban

Development

Office of Lead-Based Paint Abatement and Poisoning Prevention

\section{Objectives}

To identify and quantify factors affecting the field performance of portable $\mathrm{x}$-ray fluorescence (XRF) measurements of lead in paint and develop a laboratory test method for evaluating portable XRF devices.

\section{Problem}

Recent legislation (Housing and Community Development Act of 1992, PL102-550) will phase in requirements for lead-based paint testing for all housing built before 1978. These requirements are expected to result in several new $x$-ray fluorescence (XRF) devices. Because of health and financial liabilities are associated with these measurements, having reliable estimates of the field performance parameters of the measurements are essential (e.g., detection limits, precision, bias). For some XRF devices, research results have shown that estimates of these performance parameters obtained using existing laboratory evaluation procedures do not agree with estimates obtained using fieldacquired data. Currently, field studies are used to characterize additional devices. However, field studies are limited in several ways -- sites having parameters in the necessary ranges are difficult to find; systematic variation of the parameters is usually not possible; implementation of new technologies may be impeded; and advances in the fundamental understanding of a measurement procedure are limited. Thus, there is a need for a laboratorybased field-simulation test protocol for the reliable evaluation of portable XRF devices 
which will overcome these limitations.

\section{Approach}

During FY 1996, BFRL will:

1. Characterize and model the scientific principles controlling the performance of portable XRF device. A mathematical model embodying a fundamental understanding of the measurement process and the effects of the composition and configuration of a test specimen upon the measurement response will be developed. First, a conceptual model of the measurement process will be constructed to portray the scientific principles involved, factors likely to affect instrument response, and characterization of typical field situations about these factors.

Second, a mathematical model based on the conceptual model will be developed. The mathematical model will be used throughout the study to explore the implications of various material configurations and field conditions on instrument response and to aid interpretation of experimental results.

2. Design and carry out laboratory experiments to simulate significant field conditions, as determined by modeling, and perform a limited field study to decide whether the laboratory results are consistent with field results. Using information obtained from the literature and other sources, and from the model, a simulated field study and an actual field study will be statistically designed. The design for the laboratorybased field simulation study will include use of model wall sections each about one-meter square containing materials in configurations typical of those found in residential buildings. The laboratory experiment will be performed in a room with temperature and humidity controls that allow the conditions to be changed as needed. Field experiments will be conducted to determine whether the estimates of measurement variability obtained in the laboratory and field are consistent with each other.

3. Upon completion of the study, BFRL will write a draft standard test method for the laboratory-based evaluation of portable XRF devices and present it to ASTM Committee E06.23, Abatement of Hazards from Lead in Buildings, for standardization.

\section{Recent Results}

Developed mathematical model for predicting effects of wall materials and configurations on $\mathrm{XRF}$ instrument response. 


\section{Factors Affecting the Leaching of Lead from Lead-Based Paint Films}

\section{Principal Investigators}

Eric Byrd

Building Materials Division

301-975-5866

Mary McKnight

Building Materials Division

301-975-6714

\section{Sponsor}

U.S. Department of Housing and Urban

Development

Office of Lead-Based paint Abatement and

Poisoning Prevention

\section{Objective}

To determine the relative importance of $\mathrm{pH}$, acid type, acid concentration, surfactant concentration, lead concentration and immersion time on the leaching of lead from lead-based paint films and to recommend encapsulant performance requirements to ASTM E06.23 for encapsulation specifications.

\section{Problem}

According to a recent U.S. Department of Housing and Urban Development (HUD) report to Congress, an estimated 57 million homes in the United States contain lead-based paint. Painting the lead-based paint with an encapsulant may abate the hazards of leadbased paint. However, questions remain about what conditions lead is leached from a leadbased paint film and transported through an encapsulant film. Previous HUD-sponsored BFRL research results showed that essentially no lead is leached from lead-based paint immersed in dilute nitric acid ( $\mathrm{pH} 2$ or $\mathrm{pH} 4$ ) or from paint immersed in $\mathrm{pH} 10$ or 12 sodium or ammonium hydroxide solutions. On the other hand, practically all the lead was leached from paint immersed in 20 percent acetic acid, while a much smaller amount was leached from a 5 percent acetic acid vinegar. These results show that leaching of lead depends greatly upon the type and concentration of acid to which the lead-based paint is exposed. The leaching effects of other acids and household cleaners could leach lead.

\section{Approach}

During FY 1996, BFRL will evaluate paint having a lead content of approximately 60 percent by mass of non-volatile components. The research consists of three tasks:

1. Conduct a two level, fractional, factorial experiment to determine the relative importance of $\mathrm{pH}$, acid type, acid concentration, surfactant concentration, lead concentration and immersion time on the leaching of lead from lead-based paint films.

2. Perform investigation of other factors depending on task 1 results.

3. Determine the transport of lead through three encapsulants for at least two exposure conditions that cause lead leaching from lead-based paint films. Exposure conditions will be selected in consultation with members of ASTM Committee E06.23, Abatement of Hazards from Lead in Paint, and HUD.

\section{Recent Results}

New project 


\section{Managing Lead-Related Hazards in Housing}

\author{
Principal Investigator \\ Mary McKnight \\ Building Materials Division \\ 301-975-6714
}

\section{Sponsor}

U.S. Department of Army

The Corps of Engineers

Construction Engineering Research Laboratory

\section{Objective}

To organize DoD/Federal Agency Task Force Meetings and provide technical assistance to solve lead-related coating problems that meet regulations.

\section{Problem}

Several practices have led to potentially hazardous levels of lead in and around military family housing, including the use of leaded paints, lead in gasoline and the presence of firing ranges. Because of the hazards associated with exposure to leaded materials (dust, paint, soil, and air particulate), there is a need for the DoD and other government agencies to coordinate policy, abatement and control procedures and guidance.

\section{Approach}

During FY 1996, BFRL will continue to plan, organize and co-chair the Task Force Meetings regularly bringing together DoD experts involved in environmental, health and engineering areas and other government agencies to support development of policy, procedures, and guidance.

\section{Recent Results}

New project

\section{Technical Assistance on Lead-Based Paint Issues}

\author{
Principal Investigator \\ Mary McKnight \\ Building Materials Division \\ 301.975.6714
}

\section{Sponsor}

U.S. Department of Housing and Urban

Development

Office of Lead-based paint Abatement and Poisoning Prevention

\section{Objectives}

To provide technical assistance on lead-based paint material-related issues and measurement procedures and Chair ASTM E06.23

Committee to develop consensus standards for abatement of lead hazards in buildings.

\section{Problem}

The Office of Lead-Based Paint Abatement and Poisoning Prevention, of the U.S. Department of Housing and Urban Development (HUD) receives many requests for technical assistance on issues about abatement of hazards from leadbased paint in housing. Some of these requests concern material properties and performance of paints and coatings and material-related measurement procedures. HUD needs an overall consensus standard practice for abating hazards from lead in buildings and related structures that would include assessment and analysis of the problem, design of abatement strategy, abatement procedures and postabatement management procedures.

\section{Approach}

During FY 1996, BFRL will provide technical assistance on topics relating to the performance of paints and coatings, the abatement of hazards from lead-based paint, measurement of lead concentrations in paint films, and other material-related issues. BFRL also will provide leadership to develop the consensus standards needed for abating hazards from lead in buildings by chairing the ASTM subcommittee formed to address this need. 


\section{Recent Results}

Provided technical assistance to HUD on the 1995 revision of HUD's guidelines for abatement of lead hazards in housing and EPA's risk assessor training manual.

Developed under NIST leadership, ASTM E06.23, Abatement of Hazards from Lead in Buildings, 13 full consensus standards and 5 provisional standards.
Performance of Tape-Bonded Seams in Elastomeric Roofing Membranes

\section{Principal Investigator}

Walter Rossiter

Building Materials Division

301-975-6719

Sponsor

National Institute of Standards and Technology

\section{Objective}

To investigate the rheological properties of EPDM roofing seams bonded with butyl-based tapes and to compare their performance against seams bonded with liquid-applied adhesives.

\section{Problem}

EPDM is a non-polar material and, as such, it has excellent weathering properties, but care must be taken in adhesively bonding EPDM with itself, as required in the formation of a roofing seam. Liquid-applied butyl adhesives have excellent weathering properties, and have been extensively and successfully used in forming EPDM roofing seams since the mid1980s. However, liquid-applied adhesives contain a large volumetric fraction of solvents, which are increasingly becoming environmentally unacceptable. A replacement for these liquid-applied adhesives is butyl-based tapes. Users of EPDM roofing such as contractors and consultants have expressed concern that the rheological properties are properly evaluated and data be developed to characterize the rheological performance.

\section{Approach}

During FY 1996, BFRL will investigate the effect of various application variables and environmental factors on the rheological performance of butyl-tape systems. BFRL will expand its FY 1995 laboratory experiments to investigate the effect of a load on rheological performance by investigating the effect of application variables and environmental factors. These experiments will include temperature, pressure, rubber surface condition, and tape thickness. The research is primarily laboratorybased, statistically designed experiments complemented by field investigations. In the 
field, roofs will be inspected and tape-bonded seam samples will be obtained. Mechanical properties of the field seam specimens will be determined and compared with those of liquidadhesive bonded seams. In the laboratory, the experimentation will focus on subjecting tapebonded EPDM seam specimens to testing of the rheological performance under a range of application and environmental conditions. The seam specimens will be prepared using currently available EPDM membrane material and tape/primer systems. Additionally, seam specimens fabricated with a solvent-based butyl adhesive will be prepared as controls and included in some experiments.

The technical approach has been developed in concert with the roofing industry and government agencies having large inventories of buildings. The outcome of this collaborative effort is a CRADA between industry and government. Eight collaborators are participating including three manufacturers of EPDM roofing, two manufacturers of seam tapes, two trade associations, and one Department of Defense agency. The results of the investigation will form the basis of the development of a test protocol and recommended criteria for evaluating the long-term performance of tape-bonded seams in EPDM roofing membranes.

\section{Recent Results}

Rossiter, W. J., Lechner, J. A., Seiler, J. F., and Embree, E., "Performance of Tape-Bonded Seams of EPDM Membranes: Initial Characterization," 1 lth NIST/NRCA Conference on Roofing Technology, National Roofing Contractors Association, 1995.

\section{International Standards for Design Life of Buildings}

Principal Investigator

Geoffrey Frohnsdorff

Building Environment Division

301-975-6706

Sponsors

National Institute of Standards and Technology

The British Standards Institute, United

Kingdom

Commonwealth Scientific and Industrial

Research Organization, Australia

\section{Objective}

To develop international standards that assure the designed life of a building under anticipated environmental actions will be achieved, and to provide a basis for maintenance management.

\section{Problem}

As building technology advances, there is an increasingly strong desire to reduce the risks associated with innovation, whether due to premature deterioration resulting from the materials and components used, or the way they are incorporated into the building design. In a partial response to the need, standard methods for assessing the service life of individual materials and components have been developed in ASTM Committee E06, "Performance of Buildings." Now, methodologies for assessing the service life of individual materials and components are to be incorporated into a methodology for assessing whether a building of a given design will perform satisfactorily over its intended life, the service life. The standard is needed to provide guidance to: designers and planners on methods for assessing the expected life of a building and assuring that its design life will be met; construction engineers on how to construct a building to assure the intended life; materials and product manufacturers on information they should provide to assist assessment of the life of a building; maintenance engineers and building owners on information they will need to carry out the maintenance required to achieve the design life; 
and standards and code bodies and legislators on the need for improved standards and codes. To prepare the standard, ISO Technical Committee 59, Building Construction, established a Working Group (WG9) on the Design Life of Buildings, with ASTM Committee E06, Performance of Buildings, as the Convener. The new WG was the result of a request made by ASTM E06, Performance of Buildings, through ANSI to ISO TC59. The WG has members from Australia, Canada, Denmark, Finland, Israel, Italy, Japan, the Netherlands, Norway, Sweden, the United Kingdom, and the United States. To advise the Convener on the U.S. position, ASTM E06 set up a U.S. TAG (Technical Advisory Group) which the Convener chairs. An executive committee has been established in the WG to plan the WG's work between its annual meetings.

\section{Approach}

During FY 1996, BFRL researchers will be working with the WG to develop a guide for Planning of, and Management for, Service Life of Buildings. Its scope will be limited to the building envelope, partitions and surface finishes. British, Canadian, and Japanese documents on service life planning of buildings will be used as working documents. Building services and mechanical equipment will not be included in the first version, though the principles described will be applicable to them; it is intended to include them in a later version of the standard. As far as possible, the standard will be based on materials science, but, until the materials science basis is adequate for use in prediction of performance, reliance will be placed on empirical knowledge (from expert opinion and existing standards). The first document is expected to have five parts. Part I will provide guidance to designers and planners; Part 2 will be for material and product manufacturers; Part 3 will be directed to maintenance planners and building owners, Part 4 will be a glossary of terms, and Part 5 will present recommended formats for preparation of service life data.

\section{Recent Results}

Created a Working Group on Design Life of Buildings; held meetings in Japan (1993), Norway (1994), the Netherlands (1995), the
UK (1995) and Sweden (1996). To facilitate interaction among some complementary international committees, meetings of the WG are coordinated with those of CIB W80/RILEM 140-PSL, Prediction of Service Life of Building Materials and Components, CIB W94, Design for Durability, and, in every third year, with the International Conference on Durability of Building Materials and Components (DBMC). A workshop on standards for service life prediction, part of the DBMC, was held in Stockholm in May 1996. Developed a WG9 report on Design Life of Buildings. 


\section{BUILDING MATERIALS}

DIVISION

CONCRETE 



\section{Computer-Integrated Knowledge Systems for High-Performance Construction Materials and Systems}

\section{Principal Investigator}

S. Shyam-Sunder

Building Materials Division

(301) 975-6713

\section{Sponsor}

National Institute of Standards and Technology

\section{Objective}

To develop and demonstrate computerintegrated knowledge systems (CIKS) for highperformance construction materials and systems (HPCMS).

\section{Problem}

The President's National Science and

Technology Council has developed, with strong endorsement by the industries of construction, a set of National Construction Goals that focus on better constructed facilities and health and safety of the construction workforce. The Goals include a call for significant reduction in delivery time; reduction in operation, maintenance, and energy costs; increase in productivity and comfort; reduction in occupant related illnesses and injuries; reduction in waste and pollution; increase in durability and flexibility; and reduction in construction work illnesses and injuries. The highly fragmented nature of the industries of construction makes it difficult, if not close to impossible, for all stakeholders -- owners, architects, designers, specifiers, construction planners, site managers, facility managers, material suppliers, equipment suppliers, the labor force, trade/professional organizations, standards development organizations, public/private technology transfer centers, universities and research institutes, and government/private laboratories -- to access and use the data, information, and knowledge necessary for widespread deployment of HPCMS. Such deployment will contribute to the realization of the National Construction Goals and the specific economic and commercial benefits that derive from them. The major potential benefits of CIKS to industry, result from the accelerated and widespread deployment of HPCMS, and include: (a) reduction in project delivery time; (b) increased durability or service life; (c) reduced maintenance and repair, and/or reduced life cycle costs; (d) reductions in cost increases/ overruns and lost time from change orders, rework, and re-engineering; and (e) increased access to education and training that will raise the overall skill level of the construction workforce and help create the market pull.

\section{Approach}

During FY 1996, BFRL will, establish a meta model for the CIKS, including identification of user needs, available information technologies, and potential industry-university-government partners in building the testbed. This model is expected to be based on an open, platformindependent, architecture that uses existing information technologies, including: intelligent agents; object-oriented systems; hypermedia systems; 3-D visualization; database systems; expert systems; neural networks and learning systems; high-speed audio, video, and imaging systems; heterogeneous, distributed systems and remote data access; high-speed (gigabit) networks; standard protocols for knowledge acquisition and representation; and data standards for integrating system interfaces and promoting interoperability.

The meta model for the system will seek to integrate well-developed BFRL computer applications through the adoption of open data standards. Collaboration and synergies will be sought with other ongoing programs within NIST, industry, academia, and other Federal agencies. CIKS is expected to provide cataloging, indexing, searching, retrieval, routing, browsing, modeling and simulation, presentation systems, electronic commerce, collaborative authoring, help/training, distance learning, and security and integrity. CIKS will provide the construction industry with 1 . universal electronic access through a singlepoint to distributed data, information, and knowledge on IIPCMS; 2 . an authoritative system to design, monitor, and manage the lifecycle performance of constructed facilities; and 3. an open testbed at BFRL to build and evaluate system architectures, data standards, 
enabling information technologies, and prototype applications.

\section{Recent Results}

New project

\section{Computer-Integrated Knowledge Systems for High-Performance Construction Materials and Systems: High-Performance Concrete}

\author{
Principal Investigator \\ James R. Clifton \\ Building Materials Division \\ 301-975-6707
}

\section{Sponsor}

National Institute of Standards and Technology

\section{Objective}

To develop and demonstrate computerintegrated knowledge-based system (CIKS) for high-performance concrete for helping the construction industry improve the speed and quality of its decisions on High-Performance Construction Materials and Systems (HPCMS).

\section{Problem}

A major need for exploiting the benefits of HPCMS is providing reliable information about these materials (composition, properties, performance, and economics) quickly to the design and construction community. Owners, designers, material suppliers, and contractors benefit from access to information about HPCMS. Organizing the information in computer-integrated knowledge systems can accomplish this. A computer integrated knowledge systems for HPCMS would consist of databases, mathematical modeis, audio/visual information, standard practices and test methods, and expert reasoning, to help decision makers in using the most reliable and advanced knowledge for selecting and using HPCMS. Although research on HPCMS is still in an early stage, it is at this stage that potential users most urgently seek knowledge on its properties and performance. This project will develop and demonstrate a computer integrated knowledge system for high-performance concrete (HPC) as a contribution to a system for all HPCMS.

\section{Approach}

During FY 1996, BFRL will perform the following tasks: 
1. Define an architecture and components of the system;

2. Develop an information model addressing the needs of different users;

3. Develop methods for acquisition, representation and exchange of knowledge to be incorporated into the system;

4. Develop and demonstrate an operational system for HPC;

5. Develop database formats for cement data

Also, in this multi year project, provision for maintaining the system will be addressed. The information model will indicate knowledge that should be included, how it will be obtained, represented, exchanged, and used or interpreted. Identification of needed information will be carried out in concert with the construction industry and its customers through workshops held with potential users. Potential sources of knowledge include HPCMS projects being performed within BFRL, other agencies such as the Federal Highway

Administration and the Corps of Engineers, NSF-sponsored research, projects sponsored by trade associations, industrial studies, and foreign studies. A group of experts from industry will be invited to collaborate in the development of CIKS that meet their foreseeable needs.

The first CIKS to be developed will address High-Performance Concrete (HPC) and it will serve as a model for systems dealing with other HPCMS. The system, HYPERCON, will consist of expert systems and guidelines dealing with material design, selection of materials, processing and quality-assurance of HPC, mathematical models developed in the Building Materials Division for predicting the performance of HPCs; database formats and demonstration databases; and results of experimental studies being carried out in the BFRL research on HPCMS. Based on the experience gained last year in development of an Internet Home Page on HPCMS (describes the Building Materials Division's research and published reports electronically), reports can be downloaded in their entirety. The Home Page provides a hypertext link to a File Transfer Protocol (FTP) site for downloading mathematical models. Using this data, an expert system will be developed giving recommendations on constituents and material properties of HPC for meeting specified performance requirements. Information by other BFRL HPC projects on models for predicting the transport properties of concrete will be placed in databases and along with test methods, linked with the expert system. Also, BFRL will develop databases covering constituents of HPC, including a database on cement data compiled by BFRL's Cement and Concrete Reference Laboratory. Its database format for concrete chemical admixtures and cement will be completed.

\section{Recent Results}

Kaetzel, L., and Clifton, J., "Knowledge

Based/Expert Systems for the Construction Industry," Materials and Structures, Vol. 28, No. 177, pp. 160-174, 1995.

Struble, L., and Kaetzel, L., "Highway Concrete (HWYCON) Expert System in the Classroom," ACBM Teaching Materials Science Engineering and Field Aspects of Concrete, July 1995. 


\section{Microstructure-Property Relationships in Random Porous Materials}

\author{
Principal Investigator \\ Edward J. Garboczi \\ Building Materials Division \\ 301-975- 6708
}

\section{Sponsor}

National Institute of Standards and Technology

\section{Objective}

To develop more accurate relationships between microstructure and properties, using computer models and experiments, and to make these relationships available in mathematical form to the cement-based materials community.

\section{Problem}

The degradation of the concrete infrastructure, worth about six trillion dollars, is a national problem. The basic physical and chemical mechanisms of degradation processes in concrete need to be understood to make scientifically sound and non-empirical service life predictions. The rates of degradation processes that affect cement-based materials are controlled by microstructure-related properties like diffusivity, permeability, and elastic moduli. Fundamental computer-based models are necessary to quantitatively relate such properties to the complex microstructure of cement-based materials. Such quantitative relationships will assist in predicting the service life of new and existing concrete.

\section{Approach}

During FY 1996, BFRL will develop simulation models to predict the service life of physical property of microstructures. Digital-imagebased approach, along with Monte Carlo techniques, will be used to simulate the formation of cement-based and ceramic materials at the fundamental level. Using finite element and finite difference techniques, coupled with fast conjugate gradient solvers, software will be developed to simulate and compute physical processes and parameters. These new microstructure and property simulation algorithms then feed into the other modeling projects and are focused on the solution of specific problems. Experimental measurements are made as necessary, either at BFRL or in collaboration with other institutions like Northwestern University and Imperial College, U.K., to validate the model results.

\section{Recent Results}

Schlangen, Erik and Garboczi, Edward J., "New Method for Simulating Fracture Using a Random Geometry Lattice," International Journal of Engineering Science, (In press).

Newbauer, C. M., Garboczi, E. J., and Jennings, H. M., "A Three-phase Model of the Elastic and Shrinkage Properties of Mortar," Journal of Advanced Cement-Based Materials, Vol.4, pp. 620, 1996.

Douglas, J.F., Thorpe, M.F., and Garboczi, Edward J., "Geometrical Percolation Threshold of Overlapping Ellipsoids," Physical Review, E 52, 819-828, 1995. 


\section{Processing of High-Performance Concrete}

\author{
Principal Investigator \\ Chiara F. Ferraris \\ Building Materials Division \\ 301-975-6711
}

\section{Sponsor}

National Institute of Standards and Technology

\section{Objective}

To develop methods for measuring and predicting the effects of formulation, curing, and processing in the performance of highperformance concretes.

\section{Problem}

After many years of slow advances in concrete technology, a worldwide awareness has arisen that the performance of concrete in areas such as ease of placement, strength development, and durability can be significantly improved. This increased awareness is evident from CEB's (Commission Euro-International de Beton) Program and the high-performance concrete research activities in Japan, Norway, and Canada. In the United States, programs such as the concrete and structure's program of the Strategic Highway Research Program (SHRP) and the establishment of the NSF Center for Advanced Cement-Based Materials (ACBM) responded to the need for improved concretes and the recognition of the possibility of the development of high-performance concrete (HPC). The Civil Engineering Research Foundation (CERF) and the American Concrete Institute (ACI) have been leading the U.S. efforts in developing a comprehensive program on HPC and has prepared a proposed program that deals with high-performance construction materials, High-Performance Construction Materials and Systems: An Essential Program for America and Its Infrastructure, CERF Technical Report 93 5011, April 1993. HPC technology is sufficiently different from conventional concrete technology in that existing test methods and processing practices (e.g., mixing, consolidating and curing) may not be applicable to HPC.

\section{Approach}

During FY 1996, BFRL will develop test methods and simulation models for predicting the rheological behavior and curing of highperformance concrete. Field problems in the processing of HPC also will be identified. Work will center on: I. predicting the rheological properties of HPC; 2. Evaluating the effect of curing practices on the performance of HPC; and 3. Studying the behavior of HPC on alkalisilica reaction (ASR).

To learn if high-strength concrete has a sufficiently high tensile strength to resist the expansive stresses from destructively expansive ASR, BFRL developed a novel apparatus for measuring expansive forces in mortar bars caused by ASR. In this test, specimens are subjected to severe testing conditions to accelerate ASR and any expansive forces resulting measured using a load cell. The results show there is a need for testing the ASR of siliceous aggregates for use in HPC.

Studies on the rheological properties, will aim at developing a method for predicting the rheological properties of HPC. A method(s) will be selected and used for measuring the important rheological properties of cement pastes and mortars, of a range of compositions and water-to-cement ratios. BFRL will continue to develop hydrodynamic models for predicting the rheological properties of concentrated suspensions to include cement paste. Based on the experimental and modeling efforts, correlations between the rheological properties of cement pastes and mortars and $\mathrm{HPC}$ will be sought. If reliable correlations are obtained, this will facilitate the development of a method for predicting the rheological properties of HPC tests performed on cement pastes or mortars.

BFRL will use its new method for studying the influence of curing (temperature and humidity) on hydration of cement paste to determine the effects of moisture, temperature conditions, and time on the performance of IIPC. An essential component of this study will be an investigation of moisture movement in hardening IIPC. The results of the study on curing and the state-of-the-art report prepared 
by BFRL's Structures Division will provide an essential input to the development of the Integrated Knowledge System for HPCMS through an expert system for the curing of HPC.

\section{Recent Results}

Ferraris, C., State-of-the-art of Rheological Testing Methods, Including the Outline of a Program to Correlate Cement Paste Rheology to Mortar Rheology, NISTIR 5869, National Institute of Standards and Technology, July 1996.

Ferraris, C., Alkali-Silica Reaction and High Performance Concrete - Progress Report, NISTIR 5742, National Institute of Standards and Technology, August 1995.

\section{Three-Dimensional \\ Microstructural/Kinetic Model of Cement Hydration}

\author{
Principal Investigator \\ Dale P. Bentz \\ Building Materials Division \\ 301-975-5864
}

Sponsor

National Institute of Standards and Technology

\section{Objective}

To develop and validate a comprehensive model for cement hydration and microstructure development in three dimensions.

\section{Problem}

A lack of a basic understanding hinders predicting the properties and performance of cement-based materials of microstructural development and hydration kinetics. Progress in this area has been slow due to the complexity of the starting materials, water and a multi-size, multi-phase cement powder, and the number and the complexity of the reactions that occur during hydration.

\section{Approach}

During FY 1996, BFRL will continue development of 1) an integrated computer model for predicting the course of development of microstructure in hardening cement pastes and 2) experimental techniques to be used in model validation. Computer modeling techniques for reconstructing a threedimensional representation of multi-phase multi-size cement particles in water and the cellular automata-based hydration model have been preliminarily validated against experimental data for hydration rates, heat release, and chemical shrinkage. Using the parabolic dispersion model of Knudsen, a single relationship has been used to relate model cycles to real time for Cements 115 and 116 issued by CCRL, at three different w/c ratios investigated experimentally. The model shows further promise in its ability to predict the longer (7-day and 28-day) term compressive strength of ASTM C109 mortar cubes from 3 . day values. All studies to date have been 
performed at room temperature. Two other temperatures $\left(15^{\circ} \mathrm{C}\right.$ and $\left.35^{\circ} \mathrm{C}\right)$ will be investigated and maturity-type concepts applied to relate results at different temperatures. The hydration model will be modified to create empty pore space as chemical shrinkage occurs during hydration, so hydration under sealed conditions can be modeled and compared with experiments, to provide useful information for the ongoing research on the curing of concrete. Ultimately, the model and its real-time simulation capabilities will be part of the group's contribution to the Integrated Knowledge-Based System for HPCMS and will be distributed electronically to industry and academia through the Inorganic Group and HPCMS world-wide-web servers and other means. The model is expected to be used in studying the degree of grinding, w/c ratio, and usage of admixtures on the resultant microstructure and properties of cement-based materials.

\section{Recent Results}

Bentz, D., A Three-Dimensional Cement Hydration and Microstructure Program. I. Hydration Rate, Heat of Hydration, and Chemical Shrinkage, NISTIR 5756, National Institute of Standards and Technology, November 1995.

\section{Development of Microstructure and Permeability in Mortar and Concrete}

\author{
Principal Investigator \\ Edward J. Garboczi \\ Building Materials Division \\ 301-975-6708
}

\author{
Sponsor \\ National Science Foundation \\ Science and Technology Center for $\Lambda$ dvanced \\ Cement-Based Materials
}

\section{Objective}

To use microstructural development and interfacial zone percolation models, with transport property experiments, to predict the transport properties of cement paste and concrete.

\section{Problem}

The transport properties of cement-based materials play an important role in their durability. Understanding cement paste is fundamental, as it is the matrix material in concrete. However, the connectivity and microstructure of interfacial zones in concrete make the prediction of concrete properties from cement paste properties a non-trivial task.

Using impedance spectroscopy, it is possible to carefully study the dependence of the electrical conductivity of cement paste on its microstructure, and validate BFRL's digitalimage-based model. Applying these results to interfacial zone percolation models and transport models, originally developed for sedimentary rocks, the major transport properties of concrete can be predicted.

\section{Approach \\ During FY 1996, BFRL will apply the results of theoretical predictions for concrete transport properties using random walk conductivity simulations and effective medium theories. Experiments using impedance spectroscopy on mortar samples are being done at Northwestern University, and interpretations performed using BFRL's models. Theoretical work involving finite element codes will be performed at BFRL to predict the elastic modi of mortar and}


concrete, and examine the piezoelectric properties of cement paste and study their effect on transport properties.

\section{Recent Results}

E. J. Garboczi, L. M. Schwartz, and D.P. Bentz, "Interpretation of the Impedance Spectroscopy of Cement Paste via Computer Modeling III: Microstructural Analysis of Frozen Cement Paste," Journal of Materials Science, Vol.30, pp 5078-5086, 1995.

Garboczi, E. J., Bentz, D. P., and Schwartz, L. M., "Modeling the Influence of the Interfacial Zone on the D.C. Electrical Conductivity of Mortar," Journal of Advanced Cement-Based Materials, pp. 169-181, 1995.

\section{Characterization of Clinker, Cement, and Flaws in Cement Paste and Concrete}

\author{
Principal Investigator \\ Paul E. Stutzman \\ Building Materials Division \\ 301-975-6715
}

\section{Sponsor}

National Institute of Standards and Technology

\section{Objective}

To develop and demonstrate techniques for characterization of clinker and cement, and microstructures and flaws in high-performance concretes.

\section{Problem}

Understanding material properties of cement, cement paste and concrete, each a complex multi-phase material, depends upon the ability to quantitatively characterize their compositions and microstructures. For example, the standard method for estimating cement composition in ASTM C-150, the Bogue calculation, has long been recognized as inaccurate yet little has been done to correct this deficiency. X-ray powder diffraction (XRD) analysis has long been used for identification of crystalline materials, yet only about one percent of users apply XRD for the quantitative analysis of mixtures. This is due in part to sample processing and data collection difficulties but, primarily is due to difficulty in measurement of individual phase diffraction pattern intensities and in matching reference phases to the unknowns.

\section{Approach}

Whole-pattern fitting using DATAPLOT has been successfully applied to test mixtures in an ASTM round robin where pattern fitting data has shown greater precision than peak area measurements. Pattern weights derived from least-squares fitting of reference patterns to the mixture pattern can be related to mass fractions of each component in the mixture. This procedure is currently being tested using the NIST Reference Clinkers following a proposed ASTM Standard Test Method currently under 
development by their X-ray Powder Diffraction Task Group. An XRD analysis guide was prepared from this work and compositional analyses of CCRL cements 115 and II 6 were obtained from this method.

During FY 1996, BFRL will explore quantitative analytical methods for determining material microstructures (including flaws). Application of backscattered electron imaging in the scanning electron microscope (SEM) has allowed researchers to investigate clinker, cement, hardened cement paste, and concrete microstructures with resolutions approaching 0.1 micrometers. X-ray imaging and image analysis facilitates identification and location of phases such as monosulfate, ettringite, and gypsum besides calcium hydroxide and calcium silicate hydrate. Combined SEM/video analysis will facilitate quantitative descriptions of microstructure length scales and include measures of phase composition and distribution, porosity, and cracking.

BFRL will develop guides for microstructural characterization of cement clinker and hardened concretes. These guides will support the proposed ASTM standard test method on microscopical analysis of cement clinker, under development by the microscopy task group, and ASTM C 856, Standard Practice for Petrographic Examination of Hardened Concrete.

\section{Recent Results}

Stutzman, P.E., Guide for X-ray Powder Diffraction Analysis of Portland Clinker and Cement, NISTIR 5755, National Institute of Standards and Technology, 1996.

Stutzman, P.E., and Centeno, L., Compositional Analysis of Beneficiated Fly Ashes, NISTIR 5598, National Institute of Standards and Technology, May 1995.

Stutzman, P.E., Quantitative X-ray Powder Diffraction Methods for Clinker and Cement, NISTIR 5403, National Institute of Standards and Technology, May 1994.

\section{Transport Properties of High- Performance Concrete}

\author{
Principal Investigator \\ Nicos S. Martys \\ Building Materials Division \\ 301-975-5915
}

\section{Sponsor}

National Institute of Standards and Technology

\section{Objective}

To provide the technical basis for predicting the transport properties affecting the service life of high-performance concrete (HPC).

\section{Problem}

Before reliable service life predictions can be made for HPC structures, a fundamental understanding of the mechanisms controlling degradation of HPC is needed. The transport properties of HPC control many degradation processes. Although diffusion in HPC has received some attention, there does not exist a comprehensive body of work that encompasses all the important material properties (e.g., permeability, diffusion, and capillarity) that control the invasion of HPC by aqueous media. Experimental methods and standard procedures are needed to form a basis for reliable characterization of transport properties.

\section{Approach}

During FY 1996, BFRL will study the ingress of liquids into HPC by computer simulation and experiment. Microstructural models, based on images of authentic microstructures, will be used in simulation models to relate transport properties at a microscopic scale (or pore size scale) to the behavior of the macro structure (e.g., mortar and concrete). Experiments to validate the models will be carried out. Also, BFRL will assess the adequacy of existing test methods for measuring the transport properties of IIPC. If necessary, new test methods will be developed and submitted to $\Lambda$ STM Committee C9 on Concrete and Concrete $\Lambda$ ggregates for consideration as standard test methods.

Work will feature modeling of transport in partially saturated concrete by modifying BFRL 
simulation models for flow in saturated porous solids. Usually concrete is treated as fully saturated or completely unsaturated. The transport properties, and their simulation capabilities will be incorporated into BFRL's Integrated Knowledge System for HPC. It is expected that the results will be distributed electronically, through BFRL's home pages, and FTP Internet Site.

\section{Recent Results}

Martys, N., and Ferraris, C., "Water Sorption in Mortars and Concrete," Cement and Concrete Research (submitted for publication).

Martys, N., "Simulation of Multi-Component Fluids in Complex Three-Dimensional Geometries by the Lattice Boltzman Method." Physical Review E, Vol. 53, pp. 743-750, 1996.

Martys, N., Survey of Concrete Transport Properties and Their Measurement, NISTIR 5592, National Institute of Standards and Technology, February 1995.
Matrix Microcracking in Polymer Composites Under Environmental and Mechanical Stresses

\author{
Principal Investigator \\ Joannie W. Chin \\ Building Materials Division \\ 301-975-6815
}

\section{Sponsor}

National Institute of Standards and Technology

\section{Objective}

The objective of this project is to study chemical and mechanical changes in polymer matrix composites following exposure to UV, moisture, and mechanical loading for identifying factors contributing to matrix resin microcracking under environmental and mechanical stresses.

\section{Problem}

The polymer matrix is a fiber-reinforced composite binds and orients reinforcing fibers to carry the intended loads, protects them from handling and environment, and provides the interlaminar shear strength of the composite. However, the matrix is of ten considered the weak link in a fiber-reinforced composite system, since it may crack and craze during environmental exposure and stress application. Polymer composites in outdoor applications are susceptible to photo-initiated oxidation leading to surface degradation and/or crosslinking. Microcracks which develop in a UV-irradiated surface provide sites which could potentially initiate composite delamination, and provide pathways for rapid ingress of moisture and chemical agents. Matrix cracking also has been observed to occur in the early stages of fatigue cycling and is known to be accelerated by moisture. The propensity of a polymer to undergo microcracking is related to, among other factors, its toughness and ductility. Toughness, is affected by the moisture content and crosslink density of the matrix resin. Since moisture is ubiquitous, and the breakdown and/or build-up of crosslinks can be photolytically initiated, moisture and UV directly impact composite durability. Presently, there have been no satisfactory studies 
conducted on the effect of UV exposure on composite materials, and none which attempt to relate changes in chemistry and material properties to durability and service life. The combined effect of moisture, UV, and loading is not well-documented.

\section{Approach}

During FY 1996, BFRL will evaluate the factors which influence the chemical and mechanical properties of the polymer matrix resin and characterize them apart from the fiber reinforcement. Neat polymer films and model composites, will be exposed, under static and cyclic loads, to UV radiation and to UV radiation combined with varying levels of relative humidity. Chemical changes which take place in the unreinforced films will be characterized with Fourier transform infrared spectroscopy (FTIR), x-ray photoelectron spectroscopy (XPS) and differential scanning calorimetry (DSC). Changes in viscoelastic response, fracture toughness and residual stiffness/strength will be evaluated with dynamic mechanical thermal analysis (DMTA), static testing and fracture mechanics test methods. Scanning electron microscopy (SEM) and image analysis will be used to quantify microcracking in the polymer surface. Atomic force microscopy will be used to track the increase in crack density with time.

Results of all tests will be compared with controls which have not been environmentally exposed or loaded. Because crack formation and propagation will be different in a fiberreinforced polymer composite versus the neat polymer, information on crack density as a function of environmental exposure and mechanical stress also will be obtained on model composite systems. When correlations between exposure/loading conditions, microcracking and residual properties are obtained for neat and fiber-reinforced polymer systems, the final stage of this work will involve collaboration with organizations possessing the capability to develop models incorporating the above-mentioned elements.

\section{Recent Results}

New project

\section{Service Life Prediction of Polymer/Fiber Composites in Civil Engineering Applications}

\author{
Principal Investigator \\ Tinh Nguyen \\ Building Materials Division \\ 301-975-6718
}

\section{Sponsor}

National Institute of Standards and Technology

\section{Objective}

To develop and validate models for estimating shear strength of polymer/fiber composites and to develop an automated method for quantifying the effects of environmental conditions on the durability of polymer/fiber composites.

\section{Problem}

One of the prime concerns on the applications of polyner/fiber composites (PFC) in civil engineering is the effects of exposure environments on the durability of these materials and their components over the 50 to 75 year life spans. A particular failure mode is interfacial shear. The effect of moisture and temperatures on interfacial shear strength of composites in traditional applications have been extensively studied. However, almost no data exists on the effects of construction environmental conditions, e.g., concrete and salt water environments, and their interactions with temperatures on the interfacial failure of PFC.

\section{Approach}

During FY 1996, BFRL will develop models that predict interfacial failures of PFC under civil engineering environments. This research centers around three tasks:

1. Develop effective test method(s) to characterize interfacial failure,

2. Develop mathematical and conceptual models to predict interfacial failure, and

3. Conduct experiments to elucidate failure mechanisms and validate predictive models.

Parameters include environmental water, salt water, and simulated concrete solutions; temperature at $22^{\circ} \mathrm{C}$ and $60^{\circ} \mathrm{C}$; glass and 
carbon fibers; fiber surface treatment untreated and silane treated, and epoxy and vinyl ester resin matrices. Besides shear strength, the effects of these parameters on the thickness of the water layer at the matrix/substrate interface also will be measured Since the water layer at the fiber/matrix interface is considered to be responsible for the loss of shear strengths of PFC, data on the water thickness layer at the molecular level may provide information for predicting early failure of PFC in these environments.

\section{Recent Results}

Nguyen, T., Byrd, E., Alsheh, D., McDonough, W., and Seiler, J., "Interfacial Water and Adhesion Loss of Organic Coatings on Siliceous Substrates," Materials Research Society Symposium Proceedings, Materials Research Society, Vol.385, p. 57, 1995.

\section{Static and Fatigue Resistance of Polymer-matrix Composites Immersed in Simulated Sea Water}

\author{
Principal Investigator \\ Tinh Nguyen \\ Building Materials Division \\ 301-975-6718
}

\section{Sponsor \\ National Institute of Standards and Technology Advanced Technology Program (ATP)}

\section{Objective}

To assess the effects of various material, processing, and environmental factors on the service life of polymer-matrix composites immersed in simulated sea water and to develop mathematical models for predicting the service life of polymer-matrix composites subjected to static/fatigue loading while immersed in simulated sea water.

\section{Problem}

Polymer-matrix composites offer considerable potential for offshore applications because of their low mass, high strength, fatigue and corrosion resistance, and tailorability of mechanical properties. Realizing these potentials, NIST's Advanced Technology Program (ATP) has funded several projects to develop technologies for manufacturing polymer-matrix composites for offshore drilling and oil production. To increase the acceptance of composites in offshore applications, durability issues such as the effects of fatigue, creep, and impact on composites immersed in simulated sea water must be addressed. Fatigue damage in composites assumes a variety of forms, including matrix cracking, delamination, fiber/matrix debonding, and fiber fracture.

Fatigue durability of composites in a marine environment is of particular concern because little information exists on the combined effects of various factors such as sea water uptake, ionic diffusion, temperature, stress magnitude and ratio, loading frequency, and loading type.

\section{Approach}

During FY 1996, BFRL will characterize and analyze the progression and mechanisms of 
damage incurred by continuous members of fiber-reinforced polymeric composites subjected to static and dynamic fatigue loadings while immersed in simulated sea water. This information will form the basis for developing models to predict the service life of polymeric composites under a load in simulated sea water. Fiber-reinforced polymer-matrix composite specimens will be subjected to tensile loading while immersed in simulated sea water at different temperatures. Specimen geometry and dimensions will be similar to those described in ASTM D3039-76, Test Method for Tensile Properties of Fiber-Resin Composites. Tensile loads will include both static and dynamic loads at 50 percent and 80 percent of ultimate strengths.

Laminated and textile forms of both carbon and glass fiber-reinforced composites will be studied; both epoxy and polyester will be employed as the resin matrix. Specimens will be exposed and tested at $4{ }^{\circ} \mathrm{C}, 25^{\circ} \mathrm{C}$, and $50^{\circ} \mathrm{C}$ at a frequency of $10 \mathrm{~Hz}$. Multiple specimens will be tested at each experimental condition. For baseline data and to decouple the loading and environmental effects, specimens will also be tested under monotonic loading conditions in air and the stiffness, strength, and damage characteristics determined. Damage will be determined at regular intervals through a combination of edge replication, microscopy (SEM, and microprobe xray), and measurement of residual mechanical properties. Mass transport properties of salt and water will be measured using radioactive tracers. Based on experimental evidence, time-dependent (or load cycle-dependent) damage will be characterized by a parameter, which captures the essence of progressive damage and mechanical property degradation. The damage parameter, which may be a scalar or tensorial quantity, will be formulated as for damage patterns generated during the fatigue process. A damage evolution or progression law will be derived based on the dissipative energy principle. The service life or total number of cycles to failure will then be determined by integration of the damage evolution law to reach the critical value of the damage parameter that renders the structure useless or unserviceable.

BFRL will construct a life prediction model using a continuum damage mechanics approach coupled with a finite element technique. The theory of microcrack growth will first be developed based on attack of simulated sea water at the crack tip. Evolution of damage will be formulated based on the microcrack growth theory. The constitutive law incorporating the instantaneous damage level will be formulated taking into account the influence of the microcrack interactions on the stiffness matrix. Verification of the validity will be made against the experimental observations carried out previously.

Ultimately, a 2D, or 3D, finite-element model will be constructed to predict the service life of an off-shore substructure subjected to marine conditions. The model will be capable of handling the complex geometry of the structure. Input to the model will be loading conditions simulating the in-service environment, the initial (as received) material properties, and the validated damage evolution law. The solution scheme will be carried out in incremental time or loading steps, in analogy to an iterative scheme in nonlinear elastic/plastic analysis. At each loading step, stress, strain fields and stiffness matrix and damage parameters will be solved until the damage parameter reaches a predetermined critical value. In this way, the critical regions near the stress concentrators having the highest stresses can be monitored and ultimately the service life of the off-shore structure under various static or fatigue conditions computed.

\section{Recent Results}

New project 


\section{Spectral Ultra Violet-B Radiation Database}

\author{
Principal Investigator \\ Lawrence Kaetzel \\ Building Materials Division \\ 301-975-5912
}

\section{Sponsor}

National Institute of Standards and Technology

\section{Objective}

To develop a computerized database system of solar ultra-violet B radiation data for dissemination as a 1997 NIST Standard

Reference Data Program product.

\section{Problem}

Spectral UV-B radiation (solar) data is important to a wide range of scientific disciplines including materials (e.g., degradation of composites, plastics and coatings and color changes in coatings, and textiles). The data in the database will be invaluable input into models for predicting the service life of building materials and in assessing the relative severity of different exposure environments throughout the United States. Currently, the Smithsonian Institution, several universities, and NIST's Physics Laboratory are collecting UV-B spectral irradiance data at several locations throughout the United States. By March 1996, three more NIST sites will be added to this network making it the largest UV. radiation network in the United States.

\section{Approach}

During FY 1996, BFRL will perform four tasks:

1. Characterize the data,

2. Evaluate the quality control measures for data accuracy (e.g., calibration, transformations),

3. Develop database formats that can be used for data from different sources, and

4. Establish the database to represent outdoor weathering factors, specifically, solar spectral $U V$-B radiation data. A graphical user interface will be developed during the second year of this project.

In collaboration with NIST's Physics
Laboratory, solar spectroradiometers will be calibrated and inter-comparison data sets generated will be used to develop criteria for establishing guidelines for data set quality. Databases on the performance of coating materials will be used to develop the user interface and to test the use of the computerized database system for measuring the environmental effects on materials performance. The Steel Structures Painting Council and the BFRL's Coatings Service Life Consortium activities will be used to obtain feedback for the user interface and to test prototype systems. It is expected that this database will provide input for a wide-variety of models including those for predicting the appearance service life of coatings exposed outdoors.

\section{Recent Results}

Completed agreement to transfer solar UV-B data from the Smithsonian Institution Environmental Research Center. A graphical user interface was selected and designed for implementing the SRD product. 
Cementitious Materials Modeling Laboratory (CMML)

\author{
Principal Investigator \\ Edward J. Garboczi \\ Building Materials Division \\ 301-975-6708
}

\section{Sponsor}

National Science Foundation Center for

Advanced Cement-Based (ACBM)

Northwestern University

\section{Objective}

To advance the science of cement-based materials through the electronic availability of models and the transfer of modeling knowledge through workshops and documentation.

\section{Problem}

Many cement-based materials models that have proved themselves in research applications need to be effectively transferred to the general materials science of concrete to be of wider use. Also, such models also need to be documented so others than the developers can use them, and some researchers, especially graduate students, need extra training to be able to use the models.

\section{Approach}

During FY 1996, BFRL will make available in hypertext format, its work on cement-based material modeling. The models will be made available via anonymous file transfer protocols (ftp). Users can get the code and request a copy of the documentation. Those who need further training in the modeling techniques used in the models can attend the annual NIST/ACBM Computer Modeling Workshop to get the required training. Cooperation is maintained with RILEM Committee 123-MME on Modeling of Cement ACBM faculty, and other computer modellers around the nation.

\section{Recent Results}

Developed an anonymous ftp director and placed it on the CMML computer (titan.cbt.nist.gov), making several previously documented models available to the general research public.

\section{Testing of U.S. Capitol Pavers}

\author{
Principal Investigator \\ Chiara F. Ferrarris \\ Building Materials Division \\ 301-975-67 II
}

\section{Sponsor \\ Office of the Architect of the Capitol}

\section{Objective}

To determine the reason warping occurred on concrete pavers, installed in 1992 on the lower terrace of the Capitol Building.

\section{Problem}

The pavers are $1.22 \mathrm{~m} \times 0.61 \mathrm{~m} \times 64 \mathrm{~mm}$ and made of two layers of different concretes. They were placed in 1992 and about 2 years later they had warped. The warping caused unevenness of the walking surface and a possible trip hazard to visitors to the Capitol. There is a need to determine the causes of warping.

\section{Approach}

During FY 1996, BFRL will complete a series of tests to determine the most probable cause of concrete paver warping. Analyses include testing whole pavers in conditions simulating those in the field, measuring the length changes due to thermal or hydraulic cycles, and alkalisilca reaction (ASR). Other material characteristics will be measured including compressive and flexural strength. All tests will be done on pavers stored in a warehouse and placed in service.

\section{Recent Results}

Ferrarris, C., Stutzman, P., and Clifton, J., Warping of Terrace Pavers at the U.S. Capitol Building, NISTIR 5847, National Institute of Standards and Technology, May 1996. 

BUILDING ENVIRONMENT DIVISION

\section{COMPUTER \\ INTEGRATED \\ CONSTRUCTION}





\section{STEP for the Process Plant Industries}

Principal Investigator

Mark E. Palmer

Building Environment Division

301-975-5858

\section{Sponsor}

National Institute of Standards and Technology

\section{Objective}

To assist the U.S. process plant industries develop the Product Data Representation and Exchange (STEP) application protocols (APs)needed for exchanging and sharing information during the design and construction, and maintenance of process plants.

\section{Problem}

The U.S. process plant industries seek to improve their use of computerized systems through integration of information systems, e.g., automation of the exchange of information among systems. The many computerized systems in use can be integrated only at great cost because of their incompatible proprietary representations of information; information exchange today is accomplished largely through manual methods. Standard, neutral information representations and exchange methods are needed that allow system vendors to be innovative and yet allow system users to exchange and share information about process plants automatically. The evolving international standard ISO 10303 -Product Data

Representation and Exchange, known as STEP, is providing the base technology. STEP application protocols must be developed that meets the needs of the process plant industries.

\section{Approach}

During FY 1996, BFRL will broaden its expertise in the CAD/CAE/CAx systems used in the process plant industries and will expand the test case library for validating the draft APs and evaluating prototype implementations of the APs. BFRL will work with PlantSTEP, Inc. to advance Version 1.0 of the Plant Spatial Configuration AP (AP 227) through the ISO ballot process. BFRL will work with
PlantSTEP, Inc. to develop the Abstract Test Suite for AP 227; BFRL will work with pdXi (Process Data Exchange Institute) to document the requirements for the Process Engineering AP, (AP 231) and circulate the requirements document for review and comment by industry world wide; and will continue to co-lead the Process Plant Application Protocol Planning Project in ISO TCI 84/SC4.

BFRL will work with industry, standards development organizations, and international programs to ensure the delivery of a harmonized suite of STEP APs that meet the industries' requirements. This will include participation in or monitoring of at least four other AP projects:

- AP 221, Functional Data and Schematic

Representation (led by UK)

- AP 212, Electrotechnical Plants (led by

Siemens, Germany)

- AP 228, HVAC (led by France)

- AP 230, Building Structural Steel (led by UK)

and, potentially, the development of extensions to some AP projects. Additionally, this project will educate industry about the potential benefits of STEP and will motivate organizations, e.g., ISA (International Society for Measurement and Control), and industry to actively participate in defining requirements and reviewing draft solutions.

It is expected that this multi-year project will develop and standardize a product data model to support: 1. the selection and use of commodity items; 2 . the exchange of process engineering information; 3 . the exchange of process and piping schematics; 4 . the design of piping systems; 5 . the exchange of shape and physical arrangement information for other plant systems; and 6 . the exchange of piping system data between engineer and fabricator.

\section{Recent Results}

Burkett, W., Craig, D., Kline, S., Palmer, M., and Skeels, J., Plant Spatial Configuration Application Protocol, Version 1.0, NISTIR 965812, National Institute of Standards and Technology, 1996.

Burkett, W., Kline, S., Palmer, M., and Skeels, 
J., Group 1 for the STEP Plant Spatial

Configuration Application Protocol, NISTIR 95-

5675, National Institute of Standards and

Technology, 1995.

Palmer, M.E. and Reed, KA., 3D Piping IGES Application Protocol Version 1.1, NISTIR 4797,

National Institute of Standards and

Technology, 1994.

Beazley, W. G. And Chapman, J.B., A

Framework for Information Technology Research in

Process Plant and Related Industries, NIST-GCR-

94-657, National Institute of Standards and

Technology, 1994.

\section{AMSANT for the Process Plant Industries}

Principal Investigator

Kent A. Reed

Building Environment Division

301-975-5852

Sponsor

National Institute of Standards and Technology

\section{Objective}

To establish and use an experimental computing and communication facility 1) to provide a baseline of industrial practice in process plant engineering and construction, 2) to support the companion projects---STEP for the Process Plant Industries and Process Plant Engineering and Construction, 3) to serve as an open testbed for integration activities of industrial partners, and 4) to serve as a communications hub for all U.S. participants.

\section{Problem}

The process plant industries comprise highly competitive vendors and users of computerized systems for the design, construction, and maintenance of process plants. An open facility is needed in which these players can test proposals for integrating their computerized systems without bias or unnecessary disclosure of proprietary developments.

\section{Approach}

During FY 1996, BFRL will work with process plant industries to determine testing and demonstration scenarios for the STEP application protocols being developed. BFRL researchers will use their laboratory facilities previously established to conduct tests and to host demonstrations. Work will rely extensively on using the Internet E-mail, file, and Web servers to support the protocol developers.

\section{Recent Results}

Evaluated the EXPRESS schema developed for STEP Application Protocol 227---Plant Spatial Configuration.

Constructed data probes for use in creating STEP Exchange files containing test case data. 
Process Plant Engineering and Construction: Structural Systems

\section{Principal Investigator}

Kent A. Reed

Building Environment Division

301-975-5852

\section{Sponsor}

National Institute of Standards and Technology

\section{Objective}

To work with U.S. industry to develop a technical capability to represent and Exchange information supporting the design and construction of structural systems using internationally accepted protocols.

\section{Problem}

The U.S. construction industry seeks to improve its use of computerized systems through integration, e.g., automation of the Exchange and sharing of information among systems. The evolving international standard ISO 10303, Product Data Representation and Exchange (STEP), is providing the base technology. The U.S. structural engineering community has recognized the advantages of STEP and seeks to make STEP Exchange viable in its computerized design, analysis, and fabrication systems.

\section{Approach}

During FY 1996, BFRL will work in collaboration with the American Institute of Steel Construction to critique the European CIM Steel submission to ISO TC 184/SC 4 and will develop a U.S. position on the STEP Structural Steelwork project. During this time, BFRL will work with Carnegie Mellon University to establish a technical basis for the position. Staff will seek input from the reinforced concrete structures community.

\section{Recent Results}

Developed a steelwork design test case, reviewed, critiqued available CIM Steel documentation, and established with AISC, an AISC Task Group as the U.S. participant in the STEP Structural Steelwork project.

\section{STEP for the Building Industry}

\author{
Principal Investigator \\ William F. Danner \\ Building Environment Division \\ 301.975-5855
}

\section{Sponsor}

National Institute of Standards and Technology

\section{Objective}

To demonstrate the use of the Product Data

Representation and Exchange (STEP)

architecture and methods for the development of related application protocols (APs) for building industry sectors (e.g., process plants) that accommodates information exchange and sharing; formulate a strategy to develop coordinated application activity models (AAMs), industry application scopes, and application information requirements for related APs; and describe building industry requirements for enterprise integration and the degree to which STEP can be used to address those requirements.

\section{Problem}

Industry sectors such as process plants require suites of related APs to deal with all the data about a product over its entire life cycle. A detailed understanding of the STEP architecture and methods, the AP data specification process, and $\mathrm{AP}$ implementation are required as a basis for the development and use of suites of related APs. Principal among the issues raised concerning the STEP architecture and methods is the degree to which STEP supports business requirements that cross application protocol boundaries. A strategy is needed that allows phased and distributed development but that provides an appropriately integrated result for commerce and industry. There is considerable interest in the building industry to use information technology to facilitate enterprise integration. Most participation in STEP has been related either directly or indirectly to this interest. STEP has provided a standard for the Exchange of product data within specific application contexts specified in APs. Industry requires an assessment of the degree to which STEP APs can be used to accommodate 
enterprise integration requirements.

\section{Approach}

During FY 1996, BFRL will develop APs using the architecture and methods of STEP. These examples will be the basis for a detailed description of how related APs are developed using the architecture and methods of STEP. They also will be used to describe how related APs can be used for product data Exchange and product data sharing in typical computer system architectures. BFRL researchers will assess alternative approaches to a phased and distributed development of APs. The relative merit with respect to providing for product data integration within and among sectors of the building industry will be the principal criterion. BFRL has provided an electronic forum for worldwide discussions of building industry concerns about the use of STEP in meeting industry requirements for enterprise integration. An analysis of these discussions has provided ten principal building industry requirements. The degree to which STEP can accommodate these requirements will be undertaken with suggestions for an additional focus in areas of deficiency.

\section{Recent Results}

Contributed development of ISO 103003-13 Architecture and Methods Reference Manual including: 1 . the design principles of the STEP architecture; 2 . the conceptual framework of the architecture about its functional components; 3 . description of the standard product data constructs used in the generic model of product data; and 4. application protocol implementations.

\section{Object Oriented Implementation of STEP}

\author{
Principal Investigator \\ Kent A. Reed \\ Building Environment Division \\ 30l- 975-5852
}

Sponsor

National Institute of Standards and Technology

\section{Objective}

To work with U.S. industry to ensure proper deployment of the Product Data Representation and Exchange (STEP) in emerging software scenarios.

\section{Problem}

The U.S. construction industry seeks to improve its use of computerized systems through integration, e.g., automation of the Exchange and sharing of information among systems. Some industrial sectors have pursued the development of ISO 10303 Product Data Representation and Exchange (STEP), to enable integration at the information level. Recently, however, the members of the general building and construction industry have formed the Industry Alliance for Interoperability (IAI) to pursue the development of object class libraries, known as IFCs, to enable integration at the application programming interface level. Careful liaison is required to ensure these approaches are compatible.

\section{Approach}

During FY 1996, BFRL will work with the industry Alliance for Interoperability ResearchAdvisory Committce to define the relationship between IFCs and STEP. Researchers will prepare position papers on STEP technologies as appropriate to aid IAI and to bring IAI issues to appropriate STEP committees for resolution.

\section{Recent Results}

New project 


\section{BUILDING ENVIRONMENT}

DIVISION

HEAT TRANSFER 



\section{Development of 2-Dimensional Heat and Moisture Transfer}

\author{
Principal Investigator \\ Douglas M. Burch \\ Building Environment Division \\ 301-975-6433
}

\section{Sponsor}

National Institute of Standards and Technology

\section{Objective}

To develop a 2-dimensional personal computer model that predicts the combined transfer of heat and moisture in building construction.

\section{Problem}

BFRL has developed a "one-dimensional" personal computer model, called MOIST, that predicts heat and moisture transfer in building construction. This model inputs hourly outdoor weather data, and predicts the moisture content and temperature of the construction layers as a function of time of year. The capability of MOIST to predict the moisture accumulation within each building material as a function of time allows the user to select the most appropriate building materials and construction for a given climate. A limitation of the MOIST model is it does not address important building applications (e.g., rising damp in earth-coupled foundation walls, water leakage into basements, the effect of framing members on heat and moisture transfer, etc.). Needed is a two-dimensional model to permit building practitioners to investigate the performance of various 2-dimensional building constructions as a function of indoor and outdoor climate and ground water level. By performing multiple computer runs and varying the construction, building practitioners can learn which constructions perform well, which do not.

\section{Approach}

During FY 1996, BFRL will develop a twodimensional personal computer program that runs from a Windows 95 operating system. Work will involve two tasks:

1. An input/output processor will be developed that permits the program user to draw the building construction on the computer screen using a calibrated cursor. The program will be equipped with a database of heat and moisture property data. The user may create a new material in the database with unique property values. Clicking the mouse at various locations will establish the nodal spacing for the finite-difference solution within the construction. All data entry will be accomplished in a Windows environment. When RUN is selected from the main menu, the program will solve for the temperature and moisture content at the specified finite-difference nodes as a function of time. Moisture distribution within the construction will be displayed in a colorcoded image with each color representing a different moisture content.

2. A set of equations will be developed that perform a heat balance at the finitedifference nodes and calculate the nodal temperatures at each time step of the analysis. A separate set of equations will be developed that perform a moisture balance at the finite-difference nodes and calculate the nodal moisture contents at each time step of the analysis. Special features of the model include:

- the effect of moisture on the thermal conductivity of materials,

- latent heat associated with water evaporating from one location of the construction and condensing at another location,

- incident solar radiation onto boundary segments having an arbitrary orientation and compass azimuth,

- variable indoor relative humidity at one of the boundary segments calculated from a moisture balance of the whole building,

- moisture transfer by both diffusion and capillary flow, and

anisotropic, moisture-dependent, transport properties.

\section{Recent Results}

BFRL verified the MOIST algorithms by comparing them to a series of laboratory experiments using a calibrated hot box; added MOIST algorithms to account for ventilated cavity spaces . 


\section{Test Procedures for Advanced Thermal Insulation Products}

Principal Investigator

\author{
A. Hunter Fanney \\ Building Environment Division \\ 301-975-5864
}

\section{Sponsor}

National Institute of Standards and Technology

\section{Objective}

To evaluate thermal measurement techniques applicable to advanced insulation systems.

\section{Problem}

Advanced insulation products are being developed and introduced into the U.S. market. Examples of these products are aerogels, powder filled panels, and evacuated panels. These products offer extremely high insulating capabilities. For example, theoretical calculations reveal that a R-value approaching 100 may be achieved in a $25.4 \mathrm{~mm}$ thick evacuated panel. Current test procedures for measuring thermal conductivity are only applicable to materials that are homogenous, have planar surfaces, and have, compared with advanced insulation products, relatively poor insulating capabilities. Appropriate measurement techniques and measurement apparatus are needed to permit accurate determination of the overall thermal conductance of advanced insulation systems.

\section{Approach}

During FY 1996, BFRL will perform extensive modifications to its new calorimetric facility to permit testing of large panels and to make it less susceptible to external environmental conditions. Finite element calculations will be performed to quantify the effect of the test apparatus on the measured thermal resistance of gas-filled panels. The new calorimeter will be calibrated using specimens of known thermal conductivity. Additional CRADA(s) will be established with manufacturers of advanced insulation products to assist them in the experimental and analytical evaluation of their design concepts. BFRL's efforts in this measurement technology area will continue to assist industry in the design and evaluation of advanced insulation concepts.

\section{Recent Results}

Fanney, A. H., "Evaluation of gas-filled evacuated panel technology" Test Procedure for Advanced Insulation Panels, BETEC Conference on Superinsulation Technology, November 1995.

Fanney, A.H., "Test Procedures for Advanced Insulation Panels," Proceedings of the 1994 International CFC and Halon Alternatives Conference, 1994. 
Low-Temperature and HighTemperature Thermal Insulation Reference Materials

\section{Principal Investigator}

Robert R. Zarr

Building Environment Division

301-975-6436

\section{Sponsor}

National Institute of Standards and Technology

\section{Objective}

To establish a low- and a high-temperature guarded hot-plate laboratory for certifying thermal insulation Standard Reference Materials over an extended temperature range.

\section{Problem}

During 1995, NIST's Materials Evaluation Division, Boulder, Colorado decommissioned several apparatuses used to measure thermal conductivity. Two apparatuses were used to develop and establish thermal insulation Standard Reference Materials (SRMs) at low and high temperatures. For example, one apparatus provided SRMs of certified values of thermal resistance from $100 \mathrm{~K}$ to $330 \mathrm{~K}$ (SRMs $1450 \mathrm{~b}$ and 1451). Recognizing the necessity for this apparatus, American Society for Testing and Materials (ASTM) Committee C-16 on Thermal Insulation requested NIST consider reestablishing these measurement capabilities within BFRL.

\section{Approach}

During FY 1996, BFRL staff will arrange for transfer and transportation of the thermal conductivity apparatus to Gaithersburg. A suitable laboratory module will be modified at BFRL to accommodate the equipment. With assistance from the staff in Boulder, the lowand high-temperature guarded hot plates will be reassembled to their previous configurations.

After set up, initial shake down tests will be conducted to check the operation of the apparatus.

After completing the shake down tests, the apparatus will be extensively evaluated to assess its accuracy. The evaluation will be based on a series of experiments and theoretical analysis. Cross-checking with BFRL's one-meter guarded hot plate will provide a benchmark at room temperature. If deemed acceptable, the high temperature guarded hot plate will be included in a high-temperature interlaboratory (roundrobin) comparison organized by ASTM C-16. Results of the round robin will provide valuable data on the performance of the hightemperature guarded hot plate concerning the apparatus operated by industry. Upon completion of the evaluation, the apparatus will be available for development of SRM 1450c.

\section{Recent Results}

New project 


\section{Fibrous-Glass Thermal Insulation SRM}

Principal Investigator

Robert R Zarr

Building Environment Division

301-975-6436

\section{Sponsor}

National Institute of Standards and Technology

\section{Objective}

To renew Standard Reference Material 1450c, fibrous-glass board thermal insulation.

\section{Problem}

BFRL provided the first lot of Standard Reference Material (SRM) 1450, fibrous-glass board in 1978. Several productions runs followed. Through SRM sales at NIST, this supply has been depleted. Since BFRL has improved its apparatus for absolute measurement of thermal resistance of thick and low-density test specimens used as transfer standards, new certified values need to be established for the thermal resistance of fibrousglass board, to be designated SRM 1450c.

\section{Approach}

During FY 1996, BFRL will determine the temperature range of interest to the thermal testing community to develop SRM 1450c. Questionnaires, private discussions, and open forums at an ASTM Committee C-16 Meeting will survey the industry on the desired requirements for SRM 1450c. A small quantity of candidate material will be obtained and evaluated. The evaluation includes: material uniformity, density and thickness distribution, shot content and thermal conductivity measurements at $297 \mathrm{~K}$ using BFRL's one-meter guarded-hot-plate. To enhance uniformity, all specimens will be taken from the same production run.

The experimental design for characterizing the material will be a full factorial of 15 thermal conductivity measurements at 5 temperatures and 3 densities. BFRL will consult with NIST's Statistical Engineering Division for final comments on the experimental design. The thermal conductivity of the SRM will be certified as a polynomial function of temperature and density having at most a fourth order temperature term and second order density term. The final model will be determined from the best fit of the thermal conductivity data. Uncertainty bands will be placed on the fitted polynomial equation.

\section{Recent Results}

New project 


\section{A Photovoltaic Solar Water Heating System}

\section{Principal Investigator}

\author{
A. Hunter Fanney \\ Building Environment Division \\ 301-975-5864
}

\section{Sponsor}

National Institute of Standards and Technology

\section{Objective}

To develop computer simulations for photovoltaic solar water heating systems.

\section{Problem}

A solar water heating system employing photovoltaic solar modules has recently been patented. The photovoltaic solar hot water system offers significant improvements over solar thermal hot water systems. For example it does not require a fluid and associated piping to transport energy produced by the collectors to the storage tanks and does not require an inverter to convert the direct current supplied by the photovoltaic array to alternating current or a battery system for storage. Presently, computer simulation tools do not exist for solar photovoltaic hot water systems. Experimental data required for computer model validation exists for one geographical location, Gaithersburg, Md. The design and system components need to be optimized further to reduce the initial system cost.

\section{Approach}

During FY 1996, BFRL will award a grant to the University of Wisconsin to develop simulation tools to determine the optimum number and resistance values of the heating elements for any given geographical location. The model will provide estimates of the displaced conventional electrical energy on a daily, weekly, monthly, and yearly time scales. Predictions of electrical demand reductions and the impact on the environment through widespread use of the solar photovoltaic solar water heating system will be possible with these simulation tools.

An extensively instrumented system will be installed at the Florida Solar Energy Center (FSEC). This system will be identical to the system currently being evaluated by BFRL at its site in Gaithersburg, Md. Under sponsorship of the U.S. Air Force, two solar photovoltaic solar water heating systems will be installed at the Kadena Air Force Base in Okinawa, Japan in early 1996. The systems will be instrumented to a level required to access the solar contribution to the total hot water load. A fourth system, spon sored by Tennessee Valley Authority, will be installed in the Great Smokey National Park in 1996. These four systems will provide the data required to validate the computer simulation models.

Activity related to reducing the initial cost of a photovoltaic solar water heating system will concentrate on working with a heating element manufacturer to develop a multiple resistive assembly within a single housing and to explore the possibility of a single-tank photovoltaic solar water heating system. A design goal of the single-tank system is to ensure that an adequate supply of hot water is available during extended periods of poor weather conditions.

It is expected that the findings from this research will: 1. produce a complete understanding of the technology; 2. develop appropriate computer modeling tools and; 3 . encourage commercialization of the photovoltaic solar water heating technology by establishing links with the private sector.

\section{Recent Results}

Fanney, A. H., and Dougherty, B. P., "A Photovoltaic Solar Water Heating System," 1996 International Solar Energy Conference, ASME, March 31- April 3, 1996.

During 1994-1996, Articles on the Photovoltaic Solar Water Heating System were published in; ASHRAE Joumal, Business \& Industry, Consumer Research, Design News, Discover, Energy \& Housing Report, PC Today, Popular Science, Science News, Technology Transfer Business, The Indian Concrete Journal, The Solar Letter, and New Scientist.

Received a Patent Award, Photovoltaic Solar Water Heating System by Fanney, H. and Dougherty, 8 March 1994. 



\section{BUILDING ENVIRONMENT}

DIVISION

\section{INDOOR AIR QUALITY}



Indoor Air Quality Modeling with Computational Fluid Dynamics

\section{Principal Investigator}

Steven J. Emmerich

Building Environment Division

301-975-6459

\section{Sponsor}

National Institute of Standards and Technology

\section{Objective}

To investigate options for using a large eddy simulation computational fluid dynamics model for studying building ventilation and indoor air quality.

\section{Problem}

There are two general types of computer simulation techniques for studying airflow and contaminant distribution in buildings multizone modeling and room airflow modeling. Multizone modeling takes a macroscopic view of indoor air quality (IAQ) by evaluating average pollutant concentrations in the different zones of a building as contaminants are transported through the building and its HVAC system. Room airflow modeling takes a microscopic view of IAQ by applying a computational fluid dynamics (CFD) program to examine the detailed flow fields and pollutant concentration distributions within a room. Each is appropriate for studying different building ventilation and LAQ problems.

Several years ago, a CFD program called EXACT was developed by BFRL for studying room airflows and was applied to the prediction of ventilation system performance in an open office space. EXACT employed a k-epsilon turbulence model then considered the only practical method for modeling turbulent airflows. Recently, a CFD program has been developed at BFRL to simulate the transport of smoke and hot gases during a fire in an enclosure. This new CFD model employs highly efficient solution procedures and a technique, called large eddy simulation (LES), to eliminate the need for an empirical turbulence model. This LES-CFD model may provide an opportunity for BFRL to make a unique contribution to solving building ventilation and IAQ problems for the building industry. There is a need to examine the appropriateness of this tool for investigating ventilation and IAQ problems.

\section{Approach}

During FY 1996, BFRL will explore using a LES-CFD program to study building ventilation and IAQ. The first task of this effort will be to learn unique features of the LES-CFD model that are advantageous or disadvantageous to studying building ventilation and IAQ. Also, attention will be given to identifying additional features needed to study problems of interest.

The second task will be to learn the current state of research in the area of CFD modeling of building ventilation and IAQ through a literature review, professional contacts, and technical conferences. A focus of the task will be learning applications of interest to the building and IAQ industries. Potential applications to be explored include modeling of building material emission and adsorption of volatile organic compounds, coupling of CFD and multizone programs, and the thermal comfort performance of innovative building ventilation systems (e.g., cold air distribution).

\section{Recent Results}

New project 


\section{Chemistry and Mass Transport Modeling for Indoor Air Quality}

Principal Investigator

George Walton

Building Environment Division

301-975-6421

Sponsor

National Institute of Standards and Technology heterogeneous chemistry, adsorption and desorption of airborne contaminants from solid and liquid phase substrates, and the transport of indoor aerosols.

\section{Recent Results}

Axley, J. W., New Mass Transport Elements and Components for the NIST IAQ Model, NIST-GCR 95-676, National Institute of Standards and Technology, July 1995.

\section{Objective}

To modify the advanced airflow and contaminant dispersal model CONTAM series to include new capabilities in chemistry and mass transport modeling.

\section{Problem}

Multizone airflow and contaminant models have been developed at BFRL, including AIRNET and CONTAM86 through CONTAM93. These models were all unique at the time of their development and have been useful for the study of airflow and indoor air quality in multizone building systems.

CONTAM, and other indoor air quality models, have always been limited in their ability to model chemistry and mass transport. These physical mechanisms are taking on increasing importance in the prediction of indoor contaminant levels given the identification of the impact of specific chemical reactions that occur indoors, the increased interest in material emissions, adsorption and desorption, and the need to model gaseous air cleaning devices. Current indoor air quality models have not implemented the theory that is available to handle these issues properly. In FY 1995, BFRL awarded a contract for the development of an analysis of existing theory in chemistry and mass transport and the identification of new component models to implement in future versions of CONTAM.

\section{Approach}

During FY 1996, BFRL will implement new component models of chemical reaction and mass transport for incorporation into CONTAM. A new version of CONTAM will be developed which will have state-of-the-art capabilities to model homogenous and 
Development of LAQ Model for Designers

Principal Investigators

Andrew K Persily

Building Environment Division

301-975-6418

George Walton

Building Environment Division

301-975-6421

\section{Sponsor}

National Institute of Standards and Technology

\section{Objective}

To develop an indoor air quality design tool.

\section{Problem}

Multizone airflow and contaminant models have been developed at BFRL, including AIRNET and CONTAM86 through

CONTAM93. These models were unique at the time of their development and have been useful for the study of airflow and indoor air quality in multizone building systems. However, most indoor air quality models, including CONTAM, have been used primarily by researchers, consultants and sophisticated designers. The design community at large still needs an indoor air quality analysis program that meets their needs and is appropriate to their technical level.

\section{Approach}

During FY 1996, BFRL will begin a three-year project working with the National Research Council of Canada (NRCC) to develop an indoor air quality modeling tool for design professionals. Based on an agreement between NRCC and BFRL, this project is in the first year of a three-year effort to develop the model structure and a version of CONTAM that is compatible with this structure. The model will include three components:

- the first two components will be a VOC emission rate database and a single-zone room air motion model, both of which are being developed at NRCC, and

- the third component of the model is a multizone airflow and pollutant transport model, that will be a revised and updated version of CONTAM.

\section{Recent Results}

New project 


\section{Infiltration and Ventilation in Large Buildings}

\author{
Principal Investigator \\ Andrew K Persily \\ Building Environment Division \\ 301-975-6418
}

\section{Sponsor \\ Department of Energy \\ Office of Building Technologies}

\section{Objective}

To analyze energy impacts of large building infiltration and ventilation system airflows employing integrated building thermal analysis and network airflow analysis, and to participate in industry consensus standards development activities.

\section{Problem}

Previous research and field investigations have shown that despite current design goals, the envelopes of modern office buildings often fall short of design expectations about airtightness performance. In addition, field testing has shown that ventilation system airflows are often quite different from their design values, leading to over-and under-ventilation and increasing the pressure differences that drive envelope leakage. The existence of such envelope leakage, and ventilation system airflows that are not in accordance with design, will increase the energy loads of a building. Besides the energy penalties, envelope air leakage can also negatively impact indoor air quality, envelope material durability and occupant comfort. However, the quantitative energy implications of envelope leakage and ventilation system airflows are not known, in part due to a lack of ability of thermal analysis models to properly account for the complexities of building airflows in large, multi-zone buildings. Although sophisticated network airflow analysis programs are available, such as AIRNET developed at BFRL, thermal analysis models are currently not able to incorporate the output of such airflow models. Such an integration of thermal and airflow analysis is necessary to properly account for the energy impacts of infiltration and ventilation system airflows.

\section{Approach}

During FY 1996, BFRL will perform two tasks:

1. BFRL will perform a study of the energy impacts of infiltration and ventilation system airflows. Work will build on existing abilities and expertise in the field of large building airflow to perform a comprehensive analysis of the energy impacts of infiltration and ventilation system airflows and to assess the national impacts of energy conservation strategies in this area. This analysis will employ the building energy simulation program TRNSYS. TRNSYS is modular in nature, and there is currently a library of modules available for performing building energy simulations. These existing TRNSYS modules will be used with a new TRNSYS module based on the multi-zone airflow program AIRNET to provide the needed simulation capability. The model will be used to analyze airflow and energy use in selected prototype buildings. Two subtasks will be completed in this effort. The first centers on developing a TRNSYS module of AIRNET to in analyze the energy impacts of infiltration and ventilation in large, multizone buildings. The second subtask will develop an analysis strategy to study these energy impacts based on the TRNSYS computer simulation program and begin the analysis needed to quantify these impacts.

2. BFRL will contribute to development of an industry consensus standard and guidelines. To provide the HVAC industry with the benefits of DOE-supported research results, BFRL will assume leadership roles on ASHRAE SSPC 62 "Ventilation for Acceptable Indoor Air Quality," ASHRAE 129P "Method of Test for Ventilation Effectiveness" and ASHRAE Guideline 10 "Criteria for Achieving Acceptable Indoor Environments," and on ASTM Subcommittee E6.4I Air Leakage and Ventilation Performance and Indoor Air Quality.

\section{Recent Results}

Emmerich S. J., Persily A. K., Van Bronkhorst D. A., A Workplan to Analyze the Energy Impacts of Envelope Airtightness in Office Buildings, NISTIR 5758, National Institute of Standards and Technology, December 1995. 
Van Bronkhorst D. A., Persily A. K, Emmerich S. J., "Energy Impacts of Air Leakage in U.S. Office Buildings," 16th AIVC Conference, Air Infiltration and Venilation Centre, September 1995.

ASTM Provisional Standard P540, Guide for Using Indoor Carbon Dioxide Concentrations to Evaluate Indoor Air Quality and Ventilation, December 1995.

\section{Demonstration of Residential Ventilation Systems}

\author{
Principal Investigator \\ Andrew K Persily \\ Building Environment Division \\ 301-975-6418
}

Sponsor

Synertech Systems Corporation

\section{Objective}

To evaluate the performance of residential ventilation systems based on multizone airflow and contaminant modeling of houses in a field monitoring program.

\section{Problem}

A variety of approaches to mechanical ventilation in residential buildings exist, which control indoor contaminant levels while maintaining thermal comfort and controlling energy consumption. The New York State Energy Research and Development Administration recently sponsored a project to identify and analyze the energy and indoor air quality impacts of ventilation approaches to residential construction. This project, performed by the California Institute for Energy Efficiency and Lawrence Berkeley Laboratory, included the collection of cost and performance data for ventilation approaches from past studies and telephone surveys with HVAC contractors. These data formed the basis for detailed analyses of the most promising approaches to evaluate their ventilation characteristics, energy usage impacts and life cycle costs in new and existing buildings. The results of these analyses have identified the most effective systems in New York State and California. A Ventilation Guide discussing the issues associated with ventilation and the most appropriate system for New York State homes was developed from the results of these analyses.

The New York State Energy Research and Development Administration has moved onto the second phase of this effort in which the actual performance of the most promising ventilation systems will be evaluated in a sample of New York State homes. This second 
phase is being conducted by Synertech Systems Corporation with a number of subcontractors, including BFRL. The performance evaluation of this second phase will involve installing ventilation systems in 26 homes and performing detailed monitoring in eight of them. The monitoring will include measurements of airflow rates and selected indoor air quality parameters.

\section{Approach}

During FY 1996, BFRL will perform computer simulations of the homes with detailed monitoring with the multizone airflow and indoor air quality model CONTAM, developed by BFRL. The houses will be set-up in CONTAM, with input values determined by site measurements. Simulations will be performed for the monitoring period to determine the ability to predict the measured values. Once this ability has been established, longer term simulations will be performed to understand the overall performance of the ventilation systems, their impact of several important indoor air quality parameters, and the existence of interactions between the ventilation systems and other building systems. The results obtained from these simulations will be compared with the trends predicted during the first phase of the effort.

\section{Recent Results}

New project

\section{Indoor Air Quality Monitoring in Green Buildings}

Principal Investigators

Andrew K Persily

Building Environment Division

301-975-6418

W. Stuart Dols

Building Environment Division

301-975-5860

\section{Sponsor}

National Institute of Standards and Technology

\section{Objective}

To develop systems that monitor the environmental performance of green buildings and to implement a monitoring program in selected green buildings.

\section{Problem}

Green buildings are designed to have minimal impacts on global, local and indoor environments. A major consideration in their design is indoor air quality, primarily as to low indoor contaminant levels but also about thermal comfort. The energy consumption of the building to provide a healthy indoor environment is also an important consideration. While much effort has gone into discussions of the technical means to meet these design objectives, little effort has been devoted to evaluating the actual performance of these buildings concerning these goals. To determine whether green buildings and the associated technologies are performing as intended, field monitoring is needed in a variety of contexts. A key element to such performance evaluation is real-time monitoring of indoor environmental conditions, mechanical equipment operation and energy consumption.

\section{Approach}

During FY 1996, BFRL will continue the monitoring effort in several Green Buildings. This work is similar to that performed by BFRL last year where several buildings were evaluated for their appropriateness in the monitoring program based on the building type, mechanical system design, location and construction 
schedule. Measurements will be taken in the Audubon and Natural Resources Defense Council in New York. Longer term monitoring efforts are being pursued in the Montana State University project and the new AAAS Building in Washington, DC. In addition, a protocol will be developed and demonstrated to measure VOC source strengths in the field.

Measurements will be made in "green" and "conventional" buildings to evaluate the protocol and to determine how much success was achieved in the green buildings about material emission rates.

\section{Recent Results}

New project

\section{IAQ Analysis of Residential Ventilation Systems}

Principal Investigator

Andrew K. Persily

Building Environment Division

301- 975-6418

Sponsor

Electric Power Research Institute

\section{Objective}

To assess the indoor air quality and ventilation performance of residential ventilation systems using multizone airflow and contaminant modeling.

\section{Problem}

A variety of approaches to mechanical ventilation in residential buildings exist, which are intended to control indoor contaminant levels while maintaining thermal comfort and controlling energy consumption. BFRL recently completed a project for the U.S. Consumer Product Safety Commission (CPSC) in which many single-family residential buildings were modeled using the multizone airflow and indoor air quality program CONTAM. Simulations were performed to predict indoor pollutant levels due to a variety of sources and to assess the impact on these pollutant levels of a few indoor air quality control technologies. The technologies considered were modifications of conventional forced-air distribution systems and included enhanced particulate filtration, heat recovery ventilator installed on the return side of the system, and an outdoor air intake duct connected to the system return. The effectiveness of these controls was a function of the source characteristics, building factors, and the outdoor pollutant concentrations.

Sometimes, pollutant concentrations were reduced by as much as 70 percent. In other cases, the reductions were lower and even resulted in increased indoor concentrations. This effort contributed a significant amount of insight to issues of residential indoor air quality. However, many indoor air quality control approaches, including several promising approaches to residential ventilation that are currently being used in the field, were not 
considered in this project.

\section{Approach}

During FY 1996, BFRL will employ its

multizone airflow and indoor air quality model

CONTAM to assess the impact of a number of approaches to residential ventilation. The first task within the project will be the development of a project plan that specifies the simulations to be performed and the output analysis. The second task will include the simulations themselves, analysis of the results, comparison of the results with the results of the CPSC study, and preparation of a final report.

Based on the houses and pollutant sources used in the CPSC project, BFRL will perform CONTAM simulations to determine the indoor air quality impacts of several residential ventilation systems. The systems to be considered will include whole house exhaust systems with distributed fresh air inlets, whole house exhaust systems relying on envelope leakage for intake, and outdoor air intake ducts that operate with local exhaust systems. A number of control modes will be considered with these systems including various pre-determined schedules of operation. Besides the contaminants simulated in the CPSC study, this work will consider occupant-generated pollutants using carbon dioxide as a surrogate. Also, long term simulations (up to 1 year) will be considered as an alternative to the 24-h simulations employed previously. The results of the analyses will be analyzed for average ventilation rates, distributions of ventilation rates and contaminant concentrations, and various measures of occupant exposure to contaminants.

\section{Recent Results}

New project

\section{Carbon Monoxide Detector Location in Residential Buildings}

\author{
Principal Investigator \\ Andrew K Persily \\ Building Environment Division \\ $301-975.6418$
}

\section{Sponsor}

National Fire Protection Research Foundation

\section{Objective}

To analyze the distribution of carbon monoxide in family living units as it relates to the installation of carbon monoxide detectors.

\section{Problem}

Low-cost carbon monoxide detectors have become available for use in warning building occupants of elevated carbon monoxide levels from a variety of sources. While the use of these devices is increasing, questions exist regarding the appropriate number of detectors and their locations in a home. There also are questions about their location within the individual room. The lack of sufficient information regarding the distribution of carbon monoxide in buildings makes it difficult to develop recommendations for detector installation. Technically based recommendations are needed by the consumers who purchase and install these devices, the manufacturers who develop installation instructions, and code-writing organizations who may develop requirements for their installation.

\section{Approach}

During FY 1996, BFRL will perform Task 1 of three tasks:

1. Perform a literature review, a technical analysis of the issue, and the development of a recommended study plan that will involve experimental studies and computer modeling.

2. Perform the experimental and modeling work in single rooms.

3. Perform the experimental and modeling work in whole buildings.

The Task 1 literature review will consist of 
assembly and analysis of published literature and other work in progress related to the issues of air movement and contaminant distribution in family living units, carbon monoxide sources, and carbon monoxide concentration measurements. As part of this effort, input from the National Fire Protection Research Foundation Technical Advisory Committee (TAC) will be sought for additional materials. The analysis of this information will focus on the following issues: likely sources of carbon monoxide and their characteristics, air and contaminant distributions in family living units, building factors affecting these distributions, appropriate modeling approaches, and measurement protocols. A report on the results of the literature review will be prepared for review by the TAC.

Following completion of the literature review, a technical analysis will be performed to identify those specific factors to carbon monoxide distribution in family living units and a draft study plan recommendation will be developed for Tasks 2 and 3 . The technical analysis will synthesize the technical findings of the literature review about what is known about carbon monoxide distributions and the factors that impact these distributions. The analysis also will identify the variables to be considered in the study plan including, but not limited to, house type and configuration, HVAC system type, and carbon monoxide source characteristics. Based on this analysis, a draft study plan recommendation will be developed to make field tests of carbon monoxide distributions in a small number of family living units and to supplement these tests with simulations under a larger number of circumstances. The objective of the study will be to obtain some insight into the issue of carbon monoxide distribution within family living units and into the factors affecting these distributions. A report containing the results of the technical analysis and the draft study plan recommendation will be prepared for review by the TAC. Based on the comments received by the TAC on the draft study plan recommendation, a final study plan for Tasks 2 and 3 will be prepared.
Recent Results

New project 



\section{BUILDING ENVIRONMENT}

DIVISION

MECHANICAL SYSTEMS AND CONTROLS 



\section{Communication Protocols for Building Controls}

\section{Principal Investigator}

Steven T. Bushby

Building Environment Division

301-975-5873

\section{Sponsors}

National Institute of Standards and Technology

Department of Energy

Federal Emergency Management Program

Office of Energy Efficiency and Renewable Energy

\section{Objective}

To develop, evaluate, and produce conformance tests for communication protocol standards on the open exchange of information between equipment from different control vendors and between different levels of control in hierarchal and distributed building management systems (BMS).

\section{Problem}

Today's direct digital control (DDC) systems employ proprietary communication protocols that prevent systems supplied by different manufacturers from communicating with each other. This has resulted in "captive customers" who, upon buying a control system, are unable to upgrade or expand it without going back to the same manufacturer. This lack of communication capability between control systems made by different manufacturers also prevents the building owner from obtaining the most capable building service by not allowing him to choose, regardless of the manufacturer, the best EMCS system, the best digital controllers, the best security system, the best fire detection system, or the best telecommunications system.

\section{Approach}

During FY 1996, BFRL will perform four tasks:

1. Administer and participate with industry partners in a BACnet Interoperability Testing Consortium. The Consortium will: - assist control system manufacturers to verify the correctness and interoperability of their.proprietary BACnet

implementations;

- verify the technical soundness of the BACnet protocol;

- identify errors or omissions in the BACnet protocol specification;

- identify ambiguities in the BACnet protocol that might lead to implementations that cannot interoperate and;

- develop insight into testing requirements which should be included in a future ASHRAE standard for testing conformance to BACnet.

2. Develop Tools that can be used to establish an industry-run BACnet certification program.

3. Establish a BACnet test-bed demonstration project in a GSA building in San Francisco.

4. Initiate work on international standardization of BACnet through ISO TC 205.

5. Begin work on expanding BACnet to integrated building services, including life safety, security, and transportation.

It is expected that the results from this research will: 1 . expand the acceptance of BACnet technology into the areas of lighting control, life safety systems, security, and elevator control; 2 . achieve international standardization of the BACnet protocol; and 3. establish an industryrun BACnet certification program based on recognized testing standards.

\section{Recent Results}

Assisted ASHRAE to publish the BACnet protocol as ANSI/ASHRAE Standard 135-1995, BACnet - A Data Communication Protocol for Building Automation and Control Network, December 1996.

Created a new subcommittee of ISO TC 205 to address international standardization of building control system protocols. 


\section{Real Time Fault Detection and Diagnostics}

\author{
Principal Investigator \\ John House \\ Building Environment Division \\ 301-975-5874
}

\section{Sponsors}

National Institute of Standards and Technology

Department of Energy

Office of Energy Efficiency and Renewable Energy

Johnson Controls

\section{Objective}

To develop and demonstrate methods for controlling and performing fault detection and diagnostics (FDD) on mechanical equipment and systems in real time and to participate in the International Energy Agency (IEA) Annex 25 Committee on Real Time Simulation of HVAC - Systems for Building Optimization, Fault Detection and Diagnostics (BOFD).

\section{Problem}

Modern buildings are being designed with increasingly complex operating systems that have seemingly limitless capabilities for monitoring and controlling the conditions in the building. Building operators are not always able to monitor and process the enormous amounts of data generated. Hence, there is a need for robust FDD tools that can be used to assist the building operator and ensure that the system is operating in the manner in which it was designed. Furthermore, there is a need to develop new control strategies that can take advantage of the capabilities offered by digital control to perform system control rather than the component type of control that has traditionally been used with pneumatic controllers. The development of advanced control strategies and methods for FDD are expected to reduce energy use in buildings, improve occupant comfort and health, and reduce equipment wear.

\section{Approach}

During FY 1996, BFRL will work with industry and academia to develop and test advanced control strategies and methods for FDD.

Computer simulations using IVACSIM + will be used initially to compare the new control strategies with traditional strategies. An experimental validation of the strategies will then be performed using the VAV air handling system in the BFRL FDD Laboratory.

Depending on availability, further validation work may be performed using the Temperature Control Module (TCM) in the large environmental chamber. BFRL will also work with Purdue University to demonstrate and compare BFRL and Purdue methods for on-line fault detection and diagnostics of the VAV air handling system in the BFRL FDD Laboratory, and to apply BFRL methods to a chiller system. Annex 25 will be coming to a close in the upcoming year; however, it is anticipated that another Annex will follow with the objective to demonstrate the FDD methods developed in Annex 25 in real buildings and to develop algorithms for implementing these methods. BFRL will examine the feasibility of using the TCM as a first phase test-bed demonstration project.

In addition, a BACnet test-bed demonstration project will be implemented in a GSA operated building in San Francisco. This site will be used in future years to evaluate the benefit of energy management systems, advanced/optimal building system control strategies, and FDD methods and applications. BFRL will examine the feasibility of using this building for the second phase test-bed for the demonstration project.

It is expected that the results of this work will: 1. produce a hierarchical framework for FDD in buildings; 2 . demonstrate viability of FDD methods in real buildings by working with the other Annex 25 participants and industry; 3. develop and demonstrate advanced control strategies (including optimal control) for buildings through linkages with industry; and 4 . develop a framework for implementing FDD methods and control strategies in BACnet energy management and control systems. 


\section{Recent Results}

Lee, W.Y., Park, C., and Kelly, G.E., "Fault

Detection of an Air Handling Unit Using

Residual and Recursive Parameter Identification

Methods," ASHRAE Transactions, vol. 102, pt. 1, 1996.

Lee, W.Y., House, J.M., Park, C., and Kelly, G.E., "Fault Diagnosis of an Air Handling Unit Using Artificial Neural Networks," ASHRAE

Transactions, vol. 1, pt. 1, 1996.
Test Procedures for Furnaces, Boilers, and Integrated Appliances

\section{Principal Investigator}

Stanley T. Liu

Building Environment Division

301-975-5880

\section{Sponsor}

Department of Energy

Office of Energy Efficiency and Renewable

Energy

Office of Codes and Standards Division

\section{Objective}

To provide equitable testing and rating procedures for determining energy performance of furnaces, boilers, and integrated appliances.

\section{Problem}

In FY 1995, the furnace industry raised an issue about the calculation of the AFUE for gasfired, two-stage modulating furnaces. When applying the DOE test procedure for the AFUE value based on test results at the rated and the low firing rates, the AFUE value based on the two-stage calculation procedure was lower than the AFUE value based on the single stage calculation under the low firing rate condition. The discrepancy between the two AFUE values was from 0.5 to 1 percentage points with the single stage, low firing rate calculation giving the higher value. This was opposite to what was expected. A study of the calculation procedures is needed to find the cause of the problem and to develop possible corrections to the calculation procedures.

During FY 1994-95, BFRL assisted DOE in evaluating a petition for waiver submitted by the York International Corp. on the determination of the power burner draft factor by the optional tracer gas procedure. The units involved in the waiver request were induced draft, gas-fired, outdoor furnaces. Because the units are installed outdoors, there is no requirement for the installation of flue pipe to the flue collar during the rating test for AFUE by the existing DOE furnace test procedure. Since most outdoor furnaces now on the market are induced draft type, a reliable and repeatable 
test procedure is required in conducting the tracer gas test and incorporating it into DOE's furnace/boiler test procedure for this type of outdoor furnaces.

During FY 1995, BFRL assisted DOE evaluate public comments received after the publication in the Federal Register (in January 1995) about the proposed revision to the DOE test procedures on residentiai furnaces/boilers, vented home heating equipment and pool heaters. A revised preamble and test procedures for furnace/boiler, vented home heating equipment and pool heaters based on the public comments and DOE's instructions were transmitted to DOE. BFRL will continue to assist DOE in completing the revised test procedures so they can be published as a final rule.

\section{Approach}

During FY 1996, BFRL will perform the following tasks:

1. Continue assisting DOE publish the Final Rule for furnaces/boilers and vented home heating equipment.

2. Investigate and evaluate the various parameters in the furnace test procedure affecting the calculation of the AFUE for single and two stage modulating furnaces to reconcile the difference in AFUE values produced by the existing calculation procedure. Sensitivity studies will be conduct on the discrepance in the AFUE values between the single stage furnace and two-stage modulating furnace to determine various differences between the two types of furnaces and identify the cause of the difference and develop possible corrections to reconcile the difference in the AFUE values.

3. Conduct laboratory tests on an induced draft, outdoor furnace for the development of a tracer gas test procedure for the determination of the power burner draft factor. Tests will be performed on a test unit obtained from the York Corporation to develop the needed tracer gas procedures.

4. Assist DOE to prepare responses to Requests for Waivers on furnace/boiler and vented home heating equipment and draft Federal Register Notices of proposed Rule Making.
5. Complete recommended test procedure for evaluating the annual performance and cost of operation of Type I and Type II combined space/water heating appliances and assist DOE in preparing a Proposed Rule Making on this product.

\section{Recent Results}

Liu, S. T., Kelly, G. E., and Terlizzi, C. P., Performance Testing of a Family of Type 1

Combination Appliances, NISTIR 5626, National Institute of Standards and Technology, April 1995. 
Commercial Furnace/Boilers, Central Air Conditioners, Heat Pumps, and Water Heaters

\author{
Principal Investigator \\ Stanley Liu \\ Building Environment Division \\ 301.975 .5880
}

\section{Sponsor}

Department of Energy

Office of Energy Efficiency and Renewable

Energy

Office of Codes and Standards

\section{Objective}

To provide equitable testing and rating procedures for determining energy performance of commercial furnace/boilers, central air conditioners, heat pumps, and water heaters.

\section{Problem}

The Energy Policy Act of 1992 (EPACT) requires DOE to develop test procedures for the energy performance of certain class of commercial heating and cooling equipment and commercial hot water heaters. The Act specifies the ANSI/ASHRAE Standard 90.1-1989 as the reference document to determine the energy performance standard. ASHRAE 90.1 referenced existing industry standards for the testing of a product. For water heaters no industry standard or test procedure is referenced for unfired hot water storage tanks. ASHRAE Standing Standard Project Committee (SSPC 90.1) has addressed some unspecified or unclear procedures after ASHRAE Standard 90.1-1989 was published. Proposed updating and revision to ASHRAE 90.1 is continuing. These issues need be addressed to develop equitable test and rating procedures for the EPACT-covered commercial products.

\section{Approach}

During FY 1996, BFRL will continue to assist DOE develop, revise, and complete the draft DOE test procedures for the EPACT-covered products as required by DOE's proposed rulemaking process. As ASHRAE continues its updating of the ASHRAE 90.1, BFRL will participate and provide input on the revision process. BFRL will participate in the ASHRAE SPC 155P's effort for the development of test procedure for all type of commercial space heating boilers. The computer program for commercial boilers will be used to simulate the performance of the boilers to help in developing the test procedure. The application of the ANSI/ASHRAE Standard 94.3-1986 for unfired hot water storage tanks and a possible simpler optional procedure will be evaluated.

\section{Recent Results}

Provided letter reports to the sponsor on the various tasks including deficiencies of the ASHRAE 118.1-1993 Standard for boiler/storage tank systems. 
Test Procedures for Heat Pumps and Air Conditioners

\author{
Principal Investigator \\ Brian Dougherty \\ Building Environment Division \\ 301-975-6396
}

\section{Sponsor}

Department of Energy

Office of Energy Efficiency and Renewable

Energy

Office of Codes and Standards

\section{Objective}

To provide equitable testing and rating procedures for determining energy performance of heat pumps and air conditioners.

\section{Problem}

The Energy Policy and Conservation Act (PL 94-163) (EPCA), as amended, requires the Department of Energy (DOE) to prescribe test and rating procedures and minimum performance standards for various residential appliances. In addition, the 1987 amendment to EPCA requires analysis of any test procedure amendments to determine their effect on minimum efficiency standards. DOE has, since 1975, relied on BFRL to assist in the development of the test and rating procedures.

\section{Approach}

During FY 1996, BFRL will continue its efforts toward generating a metric version of the $\mathrm{DOE}$ central air conditioner and heat pump test procedure. Based on industry feedback to date, BFRL is attempting to structure the metric version of the DOE test procedure such that conducting the DOE procedure satisfies the ISO requirements. BFRL will continue its participation at ARI and ASHRAE meetings where metrification issues are discussed. Soonto-be-completed ETL steady-state and cyclic tests will be used to quantity how capacity and $C_{D}$ are affected when evaluated at the present $8{ }^{\circ} \mathrm{C}$ test condition versus the expected metric replacement. Many manufacturers want to have cyclic testing eliminated from the test procedure.
BFRL will complete revisions to DOE's test procedure for central heat pumps and air conditioners before the rulemaking is published, summarize the major revisions in a separate letter report, and assist DOE in resolving comments received afterwards. BFRL will generate a final version of the test procedure.

BFRL will assist DOE review comments received from its request for waiver submitted by Nordyne on their line of combined heat pump-water heating appliances and will work with DOE and Nordyne in making modifications to the Nordyne proposed method. Work includes integrating the Nordyne waiver, ASHRAE Standard 137, and the BFRL proposed test method into a single document for use by DOE in a proposed rulemaking on a combined appliance test procedure. Test data will be needed before final decisions can be made to the final content of the proposed test procedure.

BFRL will work in the area of testing and rating methods for residential thermal storage heat pumps. It is hoped that collaboration will occur with Lennox Industries and UHR, two manufacturers who recently manufactured thermal storage units.

\section{Recent Results}

Written and provided internal reports to DOE on mixed system rating methods for two coil manufacturers, Unico System and Mestek; on recommended modifications to two ASHRAE Standards (16 and 58) referenced in DOE test procedures; and on rating methods for dual-fuel heat pumps and recommendations for a workshop on dual-fuel and multifunction appliances.

Contributed significant technical information to development of ASHRAE Standard 137P; to be published following approval by ASHRAE Board of Directors. 


\section{Test Procedures for Lighting Fixtures and Systems}

\author{
Principal Investigator \\ Steve Treado \\ Building Environment Division \\ 301-975-6444
}

\section{Sponsor \\ Department of Energy \\ Office of Energy Efficiency and Renewable \\ Energy \\ Office of Codes and Standards}

\section{Objective}

To provide equitable testing and rating procedures for determining energy performance of lighting fixtures and systems.

\section{Problem}

The Energy Policy and Conservation Act (P 94163) (EPCA), as amended, required the Department of Energy (DOE) to prescribe test and rating procedures and minimum performance standards for various residential appliances. In additional, the 1987 amendment to EPCA requires analysis of any test procedure amendments to determine their effect on minimum efficiency standards. DOE has, since 1975, relied on BFRL to assist in the development of the test and rating procedures. The Energy Policy Act of 1992 (EPACT) contained many provisions relating to lighting equipment, including labeling and minimum standards for incandescent and fluorescent lamps. The implementation of these provisions required the development or specification of appropriate test procedures, and a rigorous set of definitions for covered products and exemptions.

\section{Approach}

During FY 1996, BFRL will continue to provide technical support and assist in DOE rulemaking activities. It is anticipated that particular emphasis will be required for evaluating new or modified lamp products as manufacturers begin to adapt their product lines in response to the implementation of the minimum standards for lamps. This will require interpretation of whether a new lamp is a covered product, and whether the existing test procedures are adequate for determining their performance. In addition, BFRL researchers will monitor and verify light output and energy usage of available lamps to support review and possible changes to existing fluorescent and incandescent lamp standards and support evaluation of new standards for compact fluorescent and incandescent lamps. Researchers also will monitor activities of the Lighting Collaborative related to the voluntary luminaire labeling program, and will participate in the IES Testing Procedures Committee, and support NIST's National Voluntary Laboratory Accreditation Program on Energy Efficient Lighting Equipment.

BFRL will conduct laboratory experiments to determine if and how a test procedure could be used to diagnose and characterize the electrical properties of fluorescent lamps, for identification of the lamp type instead of depending solely on manufacturers designations. The laboratory measurements would investigate methods for determining lamp type from an objective test. If feasible, a test procedure would then be developed.

The laboratory will develop a method for classifying long-life lamps based on measurements, and investigate the accommodation of long-life tungsten-halogen incandescent reflector lamps in the lamp standards program. Similarly, BFRL researchers will evaluate vibration service tungsten-halogen incandescent reflector lamps to develop a test procedure for determining or verifying their vibration resistance.

\section{Recent Results}

Test procedure for general service fluorescent and incandescent reflector lamps were refined and incorporated into DOE rule making. Refined and incorporated test procedures for general service fluorescent and incandescent reflector lamps into DOE rule making. 


\section{Plumbing Test Procedures}

\author{
Principal Investigator \\ Stephen J. Treado \\ Building Environment Division \\ 301-975-6444
}

\section{Sponsor}

Department of Energy

Office of Energy Efficiency and Renewable

Energy

Office of Codes and Standards

\section{Objective}

To develop test procedures as required for plumbing products covered under The Energy Policy Act of 1992 (EPACT).

\section{Problem}

EPACT specifies performance standards for many plumbing products, including toilets, urinals, showerheads, and faucets. The American Societies of Mechanical Engineers (ASME) test procedures for measurement of hydraulic performance area also specified and are to be adopted by the DOE. While these test procedures are in general sufficient for their intended purpose, the introduction of new plumbing products and designs, and the interpretation and application of the specific testing provisions will require $\mathrm{DOE}$ to continuously monitor these changes and their effects on the Department's test procedures for plumbing products.

\section{Approach}

During FY 1996, BFRL will continue to provide technical support and liaison with manufacturers, LBL, and related organizations regarding the test procedures and minimum standards for plumbing products. BFRL will meet with manufacturers, attend industry group meetings and review revised standards to determine whether they are more or less stringent than before. Specific activities will include supporting the ongoing DOE rulemaking, evaluating the revised ASME Al 12 18.1 for equivalency, reviewing and analyzing the revised ASME Al12 19.6, and providing general technical support.
BFRL will, if deemed necessary, conduct an assessment of the sampling plan and basic model definition to ensure statistically valid performance data without an undue burden on the manufacturers. The industry supports the testing of two units for each basic models for certification surfaces. BFRL will collect information/data from the plumbing industry and conduct work to determine the soundness of the industry proposal, or develop and refine a valid statistical sampling, as required.

\section{Recent Results}

Provided technical support, review, and oversight for DOE rulemaking activities for plumbing products. 


\section{Development of Revised Test Procedures for Compact Refrigerators}

\section{Principal Investigator}

James Kao

Building Environment Division

301-975-587 1

\section{Sponsor}

Department of Energy

Office of Energy Efficiency and Renewable

Energy

Office of Codes and Standards

\section{Objective}

To conduct investigation on small refrigerators to determine if the DOE test procedure should be revised.

\section{Problem}

DOE's obtained information from some commercial test laboratories, in the past year, showed the energy test results on compact refrigerators in volumes of $0.12 \mathrm{~m}^{3}$ or less indicated repeatability problem. However, the accuracy of this information is not certain. Further investigation to confirm or deny its validity is needed.

\section{Approach}

During FY 1996, BFRL will perform the

following tasks:

1. Inquire with manufacturers and test laboratories to determine if an energy test is a problem for refrigerators in volumes of $0.12 \mathrm{~m}^{3}$ or less.

2. Evaluate the DOE energy test procedure to determine if the test procedure causes reliability problems in energy tests for small refrigerators.

3. If appropriate, develop a plan to determine the extent of the problem. One possible approach is to test the same refrigerators at different test laboratories, such as the refrigerator manufacturer, Inchcape, CSA, and NIST (a round robin approach).

\section{Recent Results}

Completed tests on two refrigerator-freezers to determine the energy effects from room temperature, room humidity, anti-sweating heater, door openings, and inside temperature settings (for both food and freezer compartments). 


\section{Development of Revised Test Procedures for Clothes Washers}

Principal Investigator

James Kao

Building Environment Division

301-975-5871

Sponsor

Department of Energy

Office of Energy Efficiency and Renewable

Energy

Office of Codes and Standards

\section{Objective}

To provide equitable testing and rating procedures for determining energy performance of clothes washers.

\section{Problem}

The Energy Policy and Conservation Act (PL 94-163) (EPCA), as amended, requires the Department of Energy (DOE) to prescribe test and rating procedures and minimum performance standards for various residential appliances. In addition, the 1987 amendment to EPCA requires analysis of any test procedure amendments to determine their effect on minimum efficiency standards. BFRL had, since 1975, assisted DOE in the development of the test and rating procedures.

\section{Approach}

During FY 1996, BFRL will evaluate clothes washer to gather basic energy performance data. These data will be analyzed to assist in future revision of test procedures and addressing possible manufacturers' requests for waivers.

Last year, DOE announced a Proposed Rule for revising the clothes washer test procedure. In the same year, the Association of Home Appliance Manufacturers' (AHAM) proposed a revised clothes washer test procedure to be used for the $1999 \mathrm{DOE}$ test procedure revision.

Both documents added measurement requirements for water consumption, energy consumption for test cloths moisture removal, and testing of water-heating clothes washer. The AHAM proposal also incorporated certain test revisions in anticipation of new control features, such as automatic fill control, that manufacturers may adapt in their new clothes washers. BFRL will review these documents and associated public hearing comments, and make recommendations to DOE based on laboratory test results and analysis.

\section{Recent Results}

Results were provided to DOE as letter reports. 


\section{Development of Revised Test Procedures for Clothes Dryers}

\author{
Principal Investigator \\ James Kao \\ Building Environment Division \\ (301) 975-5871
}

\section{Sponsor}

Department of Energy

Office of Energy Efficiency and Renewable

Energy

Office of Codes and Standards

\section{Objective}

To provide equitable testing and rating procedures for determining energy performance of clothes dryers.

\section{Problem}

The Energy Policy and Conservation Act (PL 94-163) (EPCA), as amended, requires the Department of Energy (DOE) to prescribe test and rating procedures and minimum performance standards for various residential appliances. In addition, the 1987 amendments to EPCA requires analysis of any test procedure amendments to determine their effect on minimum efficiency standards. NIST has, since 1975, assisted DOE in the development of the test and rating procedures.

\section{Approach}

During FY 1996, BFRL will evaluate clothes dryers to gather basic energy performance data. Dryers included in these tests procedure are a conventional dryer and a condenser dryer.

These data are analyzed to assist future revision of DOE test procedures and addressing possible manufacturers' requests for waivers.

The recent development of microwave commercial clothes drying shows promising potential. If the manufacturing and marketing of similar clothes dryers for residential use appear warranted, BFRL will investigate the possibility of testing such dryers in the laboratory.

\section{Recent Results}

Results were provided to DOE as letter reports.

\section{Development of Revised Test Procedures for Dishwashers}

\author{
Principal Investigator \\ Natascha Castro \\ Building Environment Division \\ 301-975-6420
}

Sponsor

Department of Energy

Office of Energy Efficiency and Renewable

Energy

Office of Codes and Standards

\section{Objective}

To conduct tests on residential dishwashers to assess its efficiency in energy consumption and performance and determine whether revisions are warranted to upgrade the procedure.

\section{Problem}

The current DOE test procedure was developed more than ten years ago and does not have provisions for testing dishwashers with adaptive controls. Therefore, dishwashers equipped with these and other recent innovations cannot be accurately tested. The main features that contribute to improved energy performance are better thermal insulation, improved motor efficiency, better water distribution, improved food filtering, and improved fill controls (using float controls instead of timer controls). Other items that have energy reducing potential are improved water heating in water-heating dishwashers and innovative control schemes. In light of energy consumption reductions in dishwashers over the past decade, review of the current test procedure is in order.

\section{Approach}

During FY 1996, BFRL will test an adaptive control dishwasher, gathering base energy and water consumption data. Performance-based tests will be run on the conventional and innovative dishwasher using soiled test loads according to the Association of Home Appliances Manufacturers (AHAM) and IEC standards. The principal investigator will conduct a survey including a literature search of controls used in appliances to assess system operation and limitations. Work will continue 
on the test procedure and will be focused on developing a method of testing energy and water consumption of innovative dishwashers for use with the 1999 standards.

The results of this research are expected to form the basis of new significant performance-based test and rating procedures for dishwashers and further encourage manufacturers to use control features and designs that reduce energy consumption.

\section{Recent Results}

Examined AHAM and International test procedures for their respective approaches to performance-based testing and energy consumption testing.

Automated the data acquisition for energy consumption, water consumption, and other data pertinent to dishwasher analysis and procured an adaptive control dishwasher.

\section{Development of Revised Test Procedures for Pool and Spa Heaters}

\author{
Principal Investigator \\ Stan Liu \\ Building Environment Division \\ $301-975-5880$ \\ Sponsor \\ Department of Energy \\ Office of Energy Efficiency and Renewable \\ Energy \\ Office of Codes and Standards
}

\section{Objective}

To provide equitable testing and rating procedure for determining energy performance of pool and spa heaters.

\section{Problem}

In FY 1993, DOE modified the test procedure for pool and spa heaters to include a calculation of an energy factor and published the procedure in the Federal Register as a Proposed Rulemaking on August 23, 1993 for public hearing and comments. The test procedure covers only gas- and oil-fired pool heaters. It does not address an electric resistance type heater or the heat pump pool heaters that were recently introduced into the marketplace. A revised test procedure is needed to include the installation, measurements, test methods, and calculation procedures for the electric resistance type and the heat pump pool heaters.

\section{Approach}

During FY 1996, BFRL will continue to provide comments and suggestions to DOE by incorporating technical data for fossil fueled and electrical operated heaters into a revised DOE test procedure for pool and spa heaters.

\section{Recent Results}

BFRL assisted DOE evaluate public comments and prepared the draft final rule in response to the comments received.

Contributed to the draft ASHRAE SPC I46P

Standard for pool and spa heaters. 


\section{BUILDING ENVIRONMENT}

DIVISION 



\section{Thermodynamic Performance of Alternative Refrigerants and Refrigerant Mixtures}

\author{
Principal Investigators \\ Peter I. Rothfleisch \\ Building Environment Division \\ 301-975-5868
}

David A. Didion

Building Environment Division

301-975-5881

Piotr A. Domanski

Building Environment Division

301-975-5877

\section{Sponsor}

National Institute of Standards and Technology

\section{Objective}

To quantify the performance potentials and limitations of a zeotropic refrigerant mixture in a residential air-to-air heat pump.

\section{Problem}

A zeotropic mixture, as a working fluid for refrigeration machines, offers two basic

advantages: 1. a gliding saturation temperature during the phase change (i.e., evaporation and condensation) and 2 . an opportunity to vary the circulating composition of the working fluid and, thus, the system's capacity. In spite of these advantages, equipment manufacturers are wary with using zeotropic mixtures because of the difficulty in servicing the equipment after a refrigerant leak; since the vapor phase and liquid phase have different compositions, a leak of one of the phases will result in a composition change of the working fluid. The tasks covered by this work statement address these three aspects (potentials and limitations) of zeotropic mixtures in an air-conditioning system.

\section{Approach}

Before FY 1993, BFRL conducted a composition-shifting study with a passive system (i.e., the accumulator) which yielded rather limited results. During FY 1993 and FY 1994, an active distillation column was designed and constructed. This design was unique and a patent application was filed. In FY 1995, the possibility of an increased composition shift was verified in laboratory tests, and the results were reported in NISTIR 5689. The results were sufficiently good to warrant a modeling effort of a heat pump with a distillation column (it will be performed under a separate project sponsored by the Electric Power Research Institute).

During FY 1996, BFRL will perform additional tests with R-407C (R-32/125/134a (23/25/52)) to completely remove the high-boiling R-134a from the circulating stream and bring the system's capacity to its maximum limit. The results will be reported in a conference paper.

BFRL has developed several sophisticated models verified by laboratory tests and then used in various BFRL studies. In FY 1995, a theoretical model CYCLE_D was prepared for the Standard Reference Data Program (SRD) and is now distributed as Database 49. The following work is planned on two other models to make them available for general use through the SRD program:

REFLEAK; a model for simulating leak/recharge processes and for calculating the resulting change of mixture composition in the system. The model will be fully debugged and documented. It also will be equipped in a userfriendly, Visual Basic interface.

CYCLE-11; a model for determining the performance potentials and tradeoffs for different refrigerant and mixtures in different heat exchanger configurations. In the FY 1996, the existing modeling limitations for cross-flow heat exchanges will be removed and the code will be fully debugged. In FY 1997, CYCLE-1 1 will be documented, furnished in a user-friendly interface, similar to that developed for SRD 49 (CYCLE_D), and will be readied for SRD distribution. In FY 1996, BFRL will use the upgraded code to study the relative merits of cross-, counter, and parallel-flow heat transfer for low- and high-glide zeotropes. The goal of the study will be to quantify the COP potentials for zeotropes as a function of temperature glide, temperature lift, and glide non-linearity. 


\section{Recent Results}

Rothfleisch, P., A Simple Method of Composition

Shifting with a Distillation Column for a Heat

Pump Employing a Zeotropic Refrigerant Mixture,

NISTIR 5689, National Institute of Standards and Technology, July 1995.

Kim, M. S., and Didion, D., "Simulation of Isothermal and Adiabatic Leak Process of Zeotropic Refrigerant Mixtures," HVAC\&R Research, Vol. 1, No. 1, 1995.

Kim, M. S., and Didion, D., "Simulation of a Leak/Recharge Process of Refrigerant Mixtures," HVAC\&R Research, Vol. 1, No. 3, 1995.

Chi, J., Didion, D., and Domanski, P., CYCLE_D:, NIST Vapor Compression Cycle Design Program, NIST Standard Reference Database 49, National Institute of Standards and Technology, May 1995.

\section{Thermodynamic Performance of Natural Fluids as Alternative Refrigerants}

\author{
Principal Investigators \\ David A. Didion \\ Building Environment Division \\ 301-975-5881 \\ Peter I. Rothfleisch \\ Building Environment Division \\ 301-975-5868 \\ Piotr A. Domanski \\ Building Environment Division \\ 301-975-5877
}

\section{Sponsor}

National Institute of Standards and Technology

\section{Objective}

To evaluate the thermodynamic and heat transfer performance of natural fluids as a working medium for refrigeration systems.

\section{Problem}

With the ozone crisis eliminating the use of chlorine in the manufacturing of refrigerants, the industry is rapidly moving toward the use of hydrofluorocarbon compounds. This conversion has been and continues to be the most dramatic change this industry has ever faced. On the horizon looms the global warming crisis and the carbon-fluorine bond of the alternative refrigerants cause them to have a high Global Warming Potential (GWP). It is the opinion of many that "natural" fluids be used as refrigerants. That is, fluids found in nature or are in such abundance in the atmosphere already that the marginal difference the refrigerant supply would be insignificant (e.g., carbon dioxide, hydrocarbons, ammonia, water vapor). Although these fluids have been used as refrigerants in the past, they (except ammonia in industrial applications) have been disregarded for various safety or efficiency or economic reasons.

\section{Approach}

During FY 1996, BFRL will evaluate natural fluids as alternative refrigerants. This work 
requires system performance analysis via computer modeling, laboratory system (breadboard) measurements and heat transfer, particularly evaporative and condensing flows, coefficient determination. The properties of these natural fluids are radically different from halogens and significant hardware changes are required and fundamental changes to the thermodynamic cycle and processes.

The main thrust of this project is the evaluation of the concept of a water-to-water heat pump. In such a system, the refrigerant is confined in the outdoor section, and the inhabited structure is heated or cooled by a secondary refrigerant. Because of a small refrigerant charge and its location outside the house, flammable refrigerants could be used. In the early analysis stage, this concept was evaluated using a modified version of CYCLE-11. Last year a new apparatus was designed and constructed for system testing with flammable refrigerants.

During the next stage, tests will be performed at operating conditions typical for cooling and heating conditions of a ground-source heat pump. The fluids tested will include: R-22 (as a reference), propane, and propane/butane mixture. In addition, R-32/152a mixture will be evaluated because it has a low GWP and high COP. The use of a secondary refrigerant will inflict a performance penalty due to the increased temperature lifts. The goal of the study is to offset this penalty by glide-matching using zeotropic mixtures and by excellent transport properties of the selected refrigerants.

Aside of evaluation of the water-to-water heat pump concept, this project covers upgrading of an air-to-air heat pump model (HPSIM) so it could cover refrigerant mixtures comprising up to five components. Several modifications will be made to better represent newer heat transfer surfaces introduced to the marketplace in the recent years.

\section{Recent Results}

Domanski, P., Didion, D., Mulroy, W., Parise, J., "A Simulation Model and Study of Hydrocarbon Refrigerants for Residential Heat Pump Systems," IIR Conference on Natural Working Fluids in Refrigeration and Air-Conditioning, Hannover, Germany, 1994.
Calorimetric and Visual Study of Heat Transfer Enhancements with Alternative Refrigerants

\section{Principal Investigators}

Mark A. Kedzierski

Building Environment Division

301-975-5282

David A. Didion

Building Environment Division

30l-975-5881

Piotr A. Domanski

Building Environment Division

301-975-5877

\section{Sponsors}

National Institute of Standards and Technology

Department of Energy

Office of Building Technologies

Building Equipment Division

Electric Power Research Institute

Customer Services Division

Trane Company

\section{Objective}

To calorimetrically and visually investigate twophase heat transfer of alternative refrigerants.

\section{Problem}

The cost of manufacturing or operating a water chiller could be significantly reduced by improving the boiling performance of 1,1 Dichloro-2,2,2-Trifluoroethane (R123). Liquid additives can be an economical means of enhancing boiling performance. Liquid additives could make it possible to reduce manufacturing cost because a chiller would require fewer tubes to meet the same capacity. Operating costs of new or existing chillers could be reduced since the additive may improve the efficiency of chillers. Typically, binary mixtures exhibit a boiling performance degradation compared to the pure components (Shock, 1982). Consequently, liquid additives that significantly enhance boiling performance are rare. 


\section{Approach}

During FY 1996, BFRL will perform two tasks; the first will focus on additives for R123 and the second will focus on in-tube evaporation and condensation of $\mathrm{R} 22$ replacements. The first BFRL additive study showed that n-Hexane enhances the evaporator performance when added to $\mathrm{R} 123$ by $\mathrm{l}$ percent mass. The Trane Company (Glamm, 1995) verified the evaporator enhancement in a full chiller test. Trane Company researchers also observed a performance degradation in the condenser in the same chiller tests. The second BFRL study furthered the understanding of the governing enhancement mechanism. Overall, the new understanding showed that it is plausible that any hydrocarbon might enhance the boiling performance of R123. If a higher molecular weight (lower vapor pressure) hydrocarbon is used as an additive, it will have less of a tendency to travel to the condenser. Consequently, the condenser performance would be unaffected by the additive if the additive remained in the evaporator.

The first task is to measure the pool boiling heat transfer performance of R123 and mixtures of low vapor pressure hydrocarbons. The hydrocarbons to be tested as additives are: $n$ Heptane, n-Octane, n-Nonane, and n-Decane. The second task is to measure the condensing performance of the additives that enhance the heat transfer. It is expected that the condensing performance with the dilute solutions of the lower vapor pressure hydrocarbons and R123 will remain close the pure R123 performance. However, additives that enhance the boiling performance of R123 can still be used to improve cycle efficiencies even if a condensing degradation is observed. Consequently, the condensation data will be useful in determining the condenser area increase required to compensate for performance degradation.

A new condensing rig will be built of a design similar to the pool boiling rig. Both the boiling and condensing rigs permit simultaneous accurate heat-transfer-coefficients measurements and visual observations with high-speed film. Another unique aspect of the additive-boiling measurements is that a laser will be used to gather data on bubble formation.
In the second task, the scope to the in-tube condensation investigation will be measured for the local condensation heat transfer coefficient of $\mathrm{R} 22$ replacements. This research will be a companion study for last year's NIST study. Last year our group investigated the local flow boiling measurements of $\mathrm{R} 22$ replacements (R32/134a, R32/125, and R32/125/134a) in a micro-fin tube. A designer of new heat pumps can size both the evaporator and the condenser with the results of both studies.

\section{Recent Results}

Kedzierski, M., Enhancement of R123 Pool Boiling by the Addition of N-Hexane, NISTIR 5780, National Institute of Standards and Technology, March 1996.

Kedzierski, M., Calimetric and Visual Measurements of R123 Pool Boiling on Four Enhanced Surfaces, NISTIR 5732, National Institute of Standards and Technology, November 1995.

Kedzierski, M., Effect of Inclination on the Performance of a Compact Brazed Plate Condenser and Evaporator, NISTIR 5767, National Institute of Standards and Technology, November 1995.

Kedzierski, M., Kim, M.S., Single Phase Heat Transfer and Pressure Drop Characteristics of an Integral-Spine-Fin Within an Annulus, NISTIR 5454, National Institute of Standards and Technology, 1994. 
FIRE SAFETY ENGINEERING DIVISION

FIRE MODELING AND APPLICATIONS 



\section{Fire Performance Design of High- Performance Materials Used in Construction}

\author{
Principal Investigator \\ Leonard Y. Cooper \\ Fire Safety Engineering Division \\ 301-975-6880
}

\section{Sponsor}

National Institute of Standards and Technology

\section{Objective}

To develop a special-purpose version of CFAST, BFRL's Consolidated Fire and Smoke Transport Model, which focuses on structural analysis applications involving traditional and high performance construction materials.

\section{Problem}

The standard method of insuring integrity in fires of building components is based on the response of building materials to a standardtest-method fire exposure. High performance steel, concrete, organic matrix composites, and combinations of these, are being considered for use in many types of modern construction. Such materials have strength $v s$. temperature and failure characteristics that can differ substantially from those of traditional construction materials. In additions, these materials can be used in ways not considered in the development of codes which dictate their use and thus acceptability. Fires involving modern furnishings and work environments may produce exposure conditions that differ substantially from those simulated by standard tests. Further, in making the transition to performance codes for buildings it is crucial that an evaluation tool be developed that supports the performance methodology, and can do this evaluation in realistic scenarios. Finally, in determining the integrity of a particular candidate assembly in the design process itself, it will be increasingly important for industry to use modern methods of engineering analysis to assure the cost-effective fire resistance of buildings.

\section{Approach}

During FY 1996, BFRL will evaluate the performance of buildings and other structures in a fire environment. Three types of phenomena need to be simulated: 1) the fire environment and its development; 2) the thermal response of structural elements exposed to the fire environment, including loading and non-loading barriers; and 3) the structural response. The fire model CFAST, is well recognized as the leading model for calculating heat transfer through walls, ceilings, and floors with a 1 dimension analysis that assumes constant properties, not effected by the fire. To use CFAST in the evaluation of the thermal and structural response of practical barrier designs, it is important to improve the analysis of barrier thermal response by allowing for 2 dimensionality, where necessary, and for material properties which change in response to the fire.

BFRL staff will establish a catalog that includes a brief list of commonly used barrier designs and develop and incorporate into CFAST barrier heat transfer analyses associated with these designs. The analyses will use measured material properties and will be validated experimentally. An advanced version of the CFAST model will include a thermal response analysis for general barrier design geometries and other structural elements, and for comparison of designs implemented in traditional and High Performance materials. The parallel effort will include development of a means of coupling the advanced model to general structural response models. This will enhance general acceptance of CFAST in the international structural design community.

With BFRL's Structures Division staff, they will determine the most suitable model of structural systems as a test bed. The emphasis will be on reliable structural modeling with availability as a research tool. The calculation-based methods to be developed will be part of the project's contribution to BFRL's Integrated Knowledge System for High Performance Construction Materials and Systems (HPCMS) and will be distributed electronically to industry and academia through the HPCMS world-wide-web server and other means. 


\section{Recent Results}

An alternative ASTM E1 19 test method approach that accounts for the actual temperature-dependent structural properties of materials has been studied. Two possible practical barrier designs for analysis and inclusion in CFAST have been identified and are working with PCA, Forintek, and NRC Canada to obtain experimental data for use in the verifying predicted fire response of these barrier designs. One or both will be incorporated into the list of barriers.

\section{BFS Lite}

Principal Investigator

Paul A. Reneke

Fire Safety Engineering Division

301-975-6696

\section{Sponsor}

National Institute of Standards and Technology

\section{Objective}

To construct a user-friendly version of the CFAST model (FASTLite) which can be used by fire protection engineers and fire service personnel with a minimum of training.

\section{Problem}

Fire models have reached a level of sophistication and reliability that makes them useful for post fire reconstruction and design specification. Complex fire models require a fair amount of expertise and often require a substantial amount of time and effort in preparing data. These limits often preclude the use of present models in the field. One means of moving fire models into common usage is to increase the usability of the models. A more usable fire model would need to be fast, userfriendly, reliable and require limited expertise or training to use. In particular, it would allow a simple method for constructing a realistic fire. As several papers have shown, the fire is the single most important input for a zone model. In practice the fire is usually the most difficult input data to generate. This model could be used in pre-fire training and planning, and scenario reconstruction and feasibility studies.

\section{Approach}

During FY 1996, BFRL will develop an interface which uses pictures and reliable databases to allow the user to build the fire scenario by selecting burning items based on generic names and pictures (videos) of these items during burning. This work is based on BFRL's Consolidated Fore and Smoke Transport Model (CFAST), BFRL's core fire model. It has been extensively verified and is accepted in the legal community in litigation and adjudication. The model can be run to determine if a (tentative) assumed scenario results in the expected (or 
actual) outcome. An evaluation of the initial scenario can then be made leading to appropriate modifications to the scenario. This method will give confidence in the validity of the finally developed scenario. The model and user interface will be provided on CD-ROM and the data to be used will be made available through BFRL's WWW Home Page and be available for downloading to the user's computer. This should provide a reliable means to disseminate findings and for the fire community to avail themselves of the most recent technology.

\section{Recent Results}

New project

\section{Fire Model Evaluation}

\author{
Principal Investigator \\ Richard D. Peacock \\ Fire Safety Engineering Division \\ 301-975-6664
}

\section{Sponsor}

National Institute of Standards and Technology

\section{Objective}

To develop protocols to quantify the accuracy of computer-based predictive fire models and the experimental, statistical, and analytical techniques to support this method.

\section{Problem}

Users and authorities are often asked to accept building fire safety design based on fire models without accepted statements of their predictive accuracy. Key to the increased use of models in performance-based standards and elsewhere is the ability to state with confidence how close actual conditions are to those predicted by the model. The current state-of-the-art in model evaluation literature typically consists of subjective statements such as "good agreement" or "agrees well." National, through ASTM and international, through the International Council of Building Research, Studies, and Documentation (CIB) consensus on the process and research needs for model evaluation is evolving from research to date in this project. The Society of Fire Protection Engineers (SFPE) also is becoming active in this area.

\section{Approach}

During FY 1996, BFRL will address 1)

development of realistic fire descriptions for use in comparing model predictions with experimental results and 2) cooperation with other organizations supporting a continuing consensus on procedures for model evaluation, peer review of models, and sensitivity analysis. Three tasks are included:

1. Burning characteristics of products. Develop through real-scale testing, a selected set of burning characteristics for products which may become involved in fires. The measured data must be in a form which can be used by fire models. 
2. Continued analysis of complex fire model. Complete a detailed sensitivity analysis of the effects of changes in heat release rate on important calculated outputs for a complex fire model using BFRL's Consolidated Fire and Smoke Transport Model (CFAST) to provide input data for the analysis.

3. Consensus on procedures. Continue effort to foster international cooperation (specifically with ASTM, CIB, and ISO) in fire model evaluation to provide a wellaccepted framework for future efforts by NIST and others.

\section{Recent Results}

Peacock, R.D., Reneke, P.A., Forney, C.L., and

Kostreva, M.M., "Issues in Evaluation of

Complex Fire Models," submitted to Fire Safety Journal.

Peacock, R.D., Reneke, P.A., Jones, W.W., Bukoswki, R.W., and Babrauskas, V., "Concepts for Fire Protection of Passenger Rail Transportation Vehicles: Past, Present, and Future," Fire and Materials, Vol. 19, 7 1-87, 1995.

\section{Hazard Version 2}

\author{
Principal Investigator \\ Walter W. Jones \\ Fire Safety Engineering Division \\ 301-975-6887
}

\section{Sponsor}

National Institute of Standards and Technology

\section{Objective}

To plan and implement the next generation hazard methodology.

\section{Problem}

Traditional approaches to product design, product evaluation, and codes and standards development address fire safety in a piecemeal fashion - for example, evaluating heat release without considering product use, toxicity, or ignition propensity. Quantitative hazard analysis techniques have the potential of providing significant cost savings and reducing the time it takes to bring successful products to market. In addition, measures are evaluated as an interacting system, including the impact of both structure and contents. Alternative protection strategies can be studied within the hazard analysis framework to give the benefitcost relation for each. Providing these alternatives promotes the design flexibility which reduces redundancies and cost without sacrificing safety.

\section{Approach}

During FY 1996, BFRL will assemble the Hazard methodology package to include CFAST, BFRL's Consolidated Fire and Smoke Transport Model, and the new high rise egress model. The combined effort will be labeled CFAST/Hazard and will provide the functionality of both in a graphical user interface. The primary efforts will address the production of the software package itself and supporting a collaborative effort with the Navy on improving one of the physical phenomena (longitudinal flow) which is of mutual interest. The new phenomena which have been incorporated into CFAST over the past two years will be documented, the new GUI paradigm will be explained, the validation effort 
will be discussed in the writeup (including the comparison process) and the expanded egress modeling will be included.

\section{Recent Results}

Completed the Beta test version of CFAST 3.0 and the new user interface.

\section{Hybrid Model Development}

Principal Investigator

Glenn P. Forney

Fire Safety Engineering Division

301-975-2313

\author{
Sponsor \\ Department of Navy \\ Naval Research Laboratory \\ Office of Naval Technology
}

\section{Objective}

To develop a model for fire growth and smoke movement applicable to Navy Ships

\section{Problem}

The effect of corridor width and relative pressure on smoke movement is missing from BFRL's Consolidated Fire and Smoke Transport Model (CFAST). This will need to be addressed by examining various CFD concepts.

\begin{abstract}
Approach
CFAST has features that make it attractive for further development to meet the objectives of this task. In particular, it has been validated against a wide range of real-scale experimental conditions which imbue confidence in its capability. Features which have been added to address this problem include vertical flow, mechanical ventilation and compartment to compartment heat transfer.
\end{abstract}

\section{Recent Results}

Baily, J., and Tatem, P.A., Validation of Fire/Smoke Spread Model (CFAST) Using Ex-USS SHADWELL Internal Ship Conflagration Control (ISCC) Fire Tests, Naval Research Laboratory, MR 6180-95-7781, 1996. 


\section{Implementation of the FDMS Protocol}

\section{Principal Investigator}

Paul Reneke

Fire Safety Engineering Division

301-975-6696

\section{Sponsor}

National Institute of Standards and Technology other data sets. This provides for others to build their own data sets and to forward new data to us for inclusion in future releases.

\section{Recent Results}

Portier, Rebecca, W., Peacock, Richard, D., and Reneke, Paul, A., Fire Data Management System, FDMS 2.0, Internet Users Guide and Technical Documentation Addendum, National Institute of Standards and Technology, (in press)

\section{Objective}

To develop a second generation database which can accommodate all fire related data for users who collect or use building fire data.

\section{Problem}

A unified method of accessing data is desirable to aid both experimental and modeling efforts in the development of the science of fire. The Fire Data Management System (FDMS), is a format for organizing and presenting fire data obtained from bench-scale and real-scale tests and fire simulation programs. By storing available fire test values in a common format database, this data is readily available for fire protection professionals, experimentalists, building code officials, and others concerned with the design and construction of "safe" structures. A central database of test values generated from a variety of sources within the fire community needs to be developed.

\section{Approach}

During FY 1996, BFRL will provide for three applications of the database. These include: a centralized database accessible to users worldwide through a text-oriented interface on the Internet; a laboratory-dependent implementation with GUI interface for PC systems; and a CD-ROM database to be generated from the centralized database. The FDMS format database provides an open file design to accommodate future test apparatus formats. An early version of the Internet database includes Cone Calorimeter, (ASTM 1354/ISO 5660) and Eurefic Corner Tests (NT 025/ISO 9705) along with several full-scale tests including 3 room, 4 room, and multi-story structures. The plans for the GUI include the ability to edit data, and export and import to 
Study of Heat and Smoke

Movement and Their Influence on

Detector and Sprinkler Response in

Enclosed Spaces with Complex

\section{Ceiling Geometries}

Principal Investigator

Richard W. Bukowski

Fire Safety Engineering Division

301-975-6853

\section{Sponsors}

National Fire Protection Research Foundation

General Services Administration

Public Buildings Services

Office of Real Property and Management

Federal Emergency Management Agency

U.S. Fire Administration

\section{Objective}

To examine the effect of complex ceiling geometry and obstructions on the distribution of heat and smoke to optimize requirements for automatic fire detector location found in NFPA $72 \mathrm{E}$ and for automatic sprinklers as covered by NFPA 13, 13D, and 13R.

\section{Problem}

Now, recommendations for the placement of automatic fire detectors found in the NFPA Standard on Detection Devices (72E) are based either on engineering judgement or on limited experimental data. In the implementation of these data, tables and curves were developed from calculations with a zone model which are also only applicable for flat, unobstructed ceilings. Since $72 \mathrm{E}$ includes recommendations for placement of fire detectors in spaces with sloping or peaked ceilings, or with open beams and joists, some validation of these recommendations is necessary. Further, detector siting problems associated with stratification and high air movement from HVAC systems are mentioned in the standards, but only limited installation guidance is provided which are based only on judgement.

\begin{abstract}
Approach
During FY 1996, BFRL will study the effects of HVAC systems on fire detectors and sprinkler placement and activation. Work centers on validating the techniques and results obtained. In the first two years of this study, research examined the effects of fire size, fire location, ceiling height, ceiling slope, typical ceiling beam/joist configurations and the effect of stratification on the distribution of heat and smoke from the fire.
\end{abstract}

\section{Recent Results}

Developed several, novel techniques to conduct computational experiments in fire flows; results are being assimilated into national standards.

Findings from work on open joists have resulted in changes to the National Fire Alarm Code. 


\section{Balanced Design}

Principal Investigator

Richard W. Bukowski

Fire Safety Engineering Division

301-975-6853

\section{Sponsors}

General Services Administration

Public Buildings Service

Office of Real Property Management and Safety

Federal Emergency Management Agency

U.S. Fire Administration

National Concrete Masonry Association

National Electrical manufacturers Association

\section{Objective}

To quantify the operational reliability of detection, sprinklers, and compartmentation as directed by PL102-522.

\section{Problem}

A tradeoff between active and passive fire protection strategies are often made without technical foundation. As legislation mandates sprinklers and detectors in specified occupancies the role of compartmentation must be understood so that overall safety is not compromised. Thus, PL102-522 tasked BFRL to conduct this study as a joint effort of the public and private sectors.

\section{Approach}

During FY 1996, BFRL will perform three tasks to meet the objectives of this project:

1. Data Collection. Collect data as background information to enrich the data base from hotel and office occupancies and insurance, government and corporate interests. Work with industry representatives to identify other major systems users who might represent sources of field data.

2. Tradeoffs. Determine the conditions under which one or more of the systems can be reduced or eliminated without unacceptable risk of loss. What "unacceptable" means is likely to be a highly subjective measure which needs a public policy type of decision.
3. Fire-Resistive (FR) vs. Noncombustible Construction (NC). Besides the difference in fire resistance ratings, field data compiled in task 1 will be analyzed to identify other characteristics of these construction types which impact on their mitigation of risk. Given that fire resistance requirements are consistent with expected duration negating any issues about performance differences, these "reliability" aspects represent the relevant factors separating these construction types.

\section{Recent Results}

Implemented a general plan to collect and analyze the data. 


\section{Enhanced Fire Safety Evaluation System}

\author{
Principal Investigator \\ Richard W. Bukowski \\ Fire Safety Engineering Division \\ 301-975-6853
}

\section{Sponsor}

General Services Administration

Public Buildings Service

Office of Real Property Management and Safety

\section{Objective}

To advance the technical basis of the Fire Safety Evaluation System (FSES) and facilitate its evolution to a risk management tool.

\section{Problem}

Current fire safety practice within GSA is rooted in the Life Safety Code prescriptions and using the FSES for equivalency determinations. The goal of GSA's Headquarters is to move this to a risk management basis. Lacking is technical guidance and review of fire safety practices and software development of fire safety evaluation management tool.

\section{Approach}

During FY 1996, BFRL will begin to embed fire safety evaluation calculations into the FSES in place of judgmental assignment of scores for system parameters.

\section{Recent Results}

Produce and delivered to GAS a Windows version of the existing FSES.

\section{Performance Standards}

\author{
Principal Investigator \\ Richard W. Bukowski \\ Fire Safety Engineering Division \\ 301-975-6853
}

\section{Sponsor}

National Institute of Standards and Technology

\section{Objective}

To support the application of fire safety engineering principles that promote the U.S. transition to Performance Codes and Standards.

\section{Problem}

The world is making a major change from prescriptive to performance codes and standards. Many recognize the need for the United States to stay in step with these changes to remain competitive in world markets. This requires that technological changes associated with the development and application of Fire Safety Engineering (FSE) principals need to be assimilated into the U.S. system.

\section{Approach}

During FY 1996, BFRL will continue to support the transfer of advanced FSE principles into practice in the United States by working with codes and standards organizations as a means to provide the seeds of an educational system for code officials and continue to work within the U.S. codes and standards system to facilitate changes. This work will be started by introducing a performance-based replacement for a section of one of the model building codes based on BFRL modeling and measurement technology.

\section{Recent Results}

Bukowski, Richard, "Setting Performance Code Objectives," Interflam'96, Interscience Communications, London, 1996.

Bukowski, Richard, "Performance Codes: Opportunities and Challenges," National Fire Academy and NEMA Annual Meeting, National Electrical Manufacturers Association, 1995.

Bukowski, Richard, "Fire Codes for Global 
Practice," Progressive Architecture, June 1995.

Bukowski, Richard, "International Activities for Establishing Performance Codes," FORUM Symposium, Tsukuba, Japan, June 1995.

Bukowski, Richard, "Predicting the Fire Performance of Buildings," ASIAFLAM '95, March 1995.

\section{Fire Safe Elevators}

Principal Investigator

John H. Klote

Fire Safety Engineering Division

301-975-6890

\section{Sponsor}

National Institute of Standards and Technology

\section{Objective}

To develop technology allowing the elevators to be used for fire evacuation of people with mobility limitations and of the general population.

\section{Problem}

Elevators in use today are not protected against the effects of fire, and are not intended to be used for fire evacuation. However, evacuation by stairs is often not practical for people with mobility limitations. Fire safe elevators are a solution to the problem of evacuation of the disabled, and they also can be used the general population. Development of elevator fire evacuation systems requires protective features and approaches to human issues of evacuees and relevant institutions. Research is not needed concerning the protective features, except for protection from water damage due to sprinklers and fire hoses.

\section{Approach}

During FY 1996, BFRL will: 1) perform research on the effects water flow from sprinklers and fire hoses into elevator shafts, and 2) work with interested groups to refine the elevator fire evacuation concept to improve its acceptance. This work is performed as two tasks:

1. Field tests will be conducted to measure and visualize water flow in elevator shafts due to exposures of sprinklers and standing water. The measurement and visualization techniques were developed during experiments conducted last year in its large fire research facility. Flow visualization will provide a first look at the water in the shaft and around elevator cars, and this could lead to water shielding of sensitive elevator components. 
2. Staff will participate in meetings of the NFPA Means of Egress Committee where a BFRL proposal to allow fire evacuation by specially protected elevators is being considered. This participation will be aimed at assuring that committee discussions on the topic have the benefit of BFRL's extensive experience in this area.

Refinement of the elevator fire evacuation concept will also include interaction with the ASME Task Group on Elevator Use During Fires and the National Elevator Industry, Inc. (NEII).

\section{Recent Results}

BFRL's findings, submitted as a proposal to NFPA's Life Safety Code, to allow fire evacuation by specially protected elevators were accepted with minor modifications by the Means of Egress Committee. NFPA will put the modified proposal out for public comments during 1996.

A Cooperative Research and Development Agreement (CRADA) with United Technology Research Corporation and United Technology's Otis Elevator studied the concept of the fire elevator system including system performance and protection.

\section{Fire Spread in Painted Stairways}

\section{Principal Investigator}

Henri E. Mitler

Fire Safety Engineering Division

301-975-6886

\section{Sponsor}

Federal Emergency Management Agency

U.S. Fire Administration

\section{Objective}

To understand and evaluate the problem of stairwell fires.

\section{Problem}

Officials in the New York City Housing Authority (NYCHA) and also in their Fire Department (NYCFD) have observed an increase in the number of fires occurring in stairwells of buildings under the care of the NYCHA. Typically, some rubbish, including discarded furniture, was placed on a landing and ignited. This produced fires of unusual severity, which travel rapidly up the stairwell for many floors, sometimes to the roof bulkhead. It has been surmised that multiple layers of paint on the walls supplied a major part of the fuel and contributed to the spread of flame up the stairwell. Such fires pose a hazard to life, and the question is what can be done to mitigate or prevent them.

\section{Approach}

During FY 1996, BFRL will study the fire spread characteristics of multiple layers of the paints used by NYC. A mockup will be constructed to simulate a staircase shaft and carry out a series of flame spread tests, varying the painted layers in various ways.

\section{Recent Results}

New project 


\section{Experimental Application of Fire Hazard Analysis for U.S. Passenger Train Systems}

\author{
Principal Investigator \\ Richard D. Peacock \\ Fire Safety Engineering Division \\ 301-975-6664
}

\section{Sponsor \\ Department of Transportation \\ Federal Railroad Administration \\ Volpe Transportation Systems Center}

\section{Objective}

To demonstrate the practicality and effectiveness of new generation test methods and hazard analysis techniques when applied to passenger rail transportation vehicle fire safety.

\section{Problem}

Considerable advances in fire safety engineering have been made in the decade since the original development of the current U.S. guidelines for passenger train material selection. Some requirements for system design, materials control, detection, suppression, and emergency egress are included in the variety of requirements - with each applying to distinct subsets of passenger-guided ground transportation. Better understanding of the underlying phenomena governing fire initiation and growth have led to the development of a new generation of test methods that can better predict the real-scale burning behavior of materials and assemblies. While, advances in fire and hazard modeling are leading a revolution in the analysis of a material's overall contribution to fire hazards in a particular application. Such an approach allows evaluation of factors besides material flammability, and of tradeoffs in the fire-safe design of the entire fire safety system. These advances should be incorporated in future designs of passenger trains.

\section{Approach}

During FY 1996, BFRL will perform full-scale tests and computer modeling to perform a fire hazard analysis of a passenger rail vehicle constructed of currently used materials and construction techniques. Mockups of passenger vehicle components will be tested in full-scale to obtain information for modeling efforts.

Currently available computer-based methods for fire hazard analysis will be employed to study specific fire scenarios, including an interior fire, exterior fire, and an interior fire on a vehicle in a tunnel. At a minimum, the analysis will consider vehicle design, materials of construction, detection and suppression systems, communications systems, and emergency egress and access. BFRL will consult with Amtrak to provide input on existing and planned vehicle designs and configurations.

A technical report will document the hazard analysis, describe the findings and conclusions of this analysis, and include explanation of the relative impact on vehicle fire safety for the range of overall system design parameters studied.

\section{Recent Results}

Performed bench-scale heat release rate testing to characteristic material performance in modern test methods to compare with earlier generation test methods from the current guidelines.

Peacock, Richard, D., Bukowski, Richard, W., Jones, Walter, W., Reneke, Paul, A., Babrauska, Vito, and Brown, James, Fire Safety of Passenger Trains, A Review of Current Approaches and new Concepts, NIST Technical Note 1406, National Institute of Standards and Technology, 1994. 
Fire Research Information Services (FRIS)

\section{Principal Investigator}

Nora H. Jason

Fire Safety Engineering Division

301-975-6862

\section{Sponsor}

National Institute of Standards and Technology

\section{Objective}

To serve BFRL staff and the broad BFRL user community while serving as a model for information resource management for the next century. This resource management system will be expanded into data dissemination through FDMS and other protocols.

\section{Problem}

To disseminate BFRL documents electronically to the user community concomitant with making pertinent information available without jeopardizing the availability and usability of the documents.

\section{Approach}

During FY 1996, BFRL will continue to provide electronic access to its documents and software through the FIREDOC database, FSBBS bulletin board and BFRL Home Page and will be investigating methods of capturing information that will facilitate the creation of a remote access system to full documents. The Construction Industry Institute (CII) publications are available from BFRL and new documents are added as received. Liaison is maintained with ICHIEFS and other database system managers.

\section{Recent Results}

Martin, Phyllis and Jason, Nora, Building and Fire Research Publications, 1994, NIST SP 838-9, National Institute of Standards and

Technology, October 1995.

\section{Fire Forum}

Principal Investigator

Richard W. Bukowski

Fire Safety Engineering Division

301-975-6853

\section{Sponsor}

National Institute of Standards and Technology

\section{Objective}

To perform the duties of Secretary to the FORUM for International Cooperation on Fire Research.

\section{Problem}

The FORUM is an international body of the Heads of fire research organizations around the world, chaired by BFRL. Sixteen members representing 14 countries participate; several new members are expected before the next annual meeting. The group requires the technical support of a trained professional to perform the day-to-day activities required to ensure effective international cooperation.

\section{Approach}

During FY 1996, BFRL will produce a meeting report for internal distribution and an annual report for general distribution. The Secretary also will disseminate information on the FORUM and its activities to appropriate organizations and to the public through INTERNET and printed media. The Secretary will assure that assignments made at the annual meeting are completed on schedule.

\section{Recent Results}

Bukowski, Richard, W, Ed., 1995 Annual

Meeting Notes, Forum for International

Cooperation on Fire Research, 1995. 
Computing and Network Resources

Principal Investigator

James W. Raines

Fire Safety Engineering Division

301-975-6855

\section{Sponsor}

National Institute of Standards and Technology

\section{Objective}

To provide improvements, day-to-day support and long range planning for the BFRL network and associated computing environment.

\section{Problem}

Efficient computing requires a uniform and consistent environment for both scientific and administrative services. As science and engineering evolve toward more collaborative efforts, a faster and more robust local area network is essential.

\section{Approach}

During FY 1996, BFRL will continue to provide the best network system possible, including, speed, reliability and compatibility. The thrust will be to obtain and maintain the fastest and most reliable LAN system consistent with ease of use and minimum disruption. Work centers on configuring, purchasing and installing new hardware and operating sof tware for the local area networks to provide a uniform and consistent system. LAN system hardware and software will be upgraded, and connection to the new fiber-optic backbone hub concentrators will provide a means for high speed (100 mbs) access to high end workstations.

\section{Recent Results}

Increased the throughput of LAN and shared resources; downtime has been kept to a minimum; started installing the planned evolution to the high speed (100 mbs) network; and provided increased emphasis on general network and computer related help for those who needed and/or requested it. 


\section{RECENT GRANTS - FIRE MODELING AND APPLICATIONS}

\section{Interactive Virtual Environments for Fire Simulation}

\author{
Principal Investigator \\ Carlos H. Sequin, Ph.D. \\ Computer Science Division, EECS Department \\ University of California, Berkeley
}

\section{Sponsor}

National Institute of Standards and Technology

\section{Objective}

To combine Berkeley virtual reality architectural walk-through technology with NIST CFAST fire simulation technology, providing simulation and scientific visualization of the CFAST model within a real-time, interactive virtual environment.

\section{Problem}

Virtual reality techniques developed at Berkeley over the last 5 years provide intuitive, real-time visualization and interaction with conditions and structures in heavily occluded architectural environments. NIST's CFAST fire simulation software provides fast, accurate computation of conditions within buildings in fire situations, but lacks the ability to construct simulation scenarios interactively or directly to visualize results in 3D. A marriage of these technologies will bring interactive $3 \mathrm{D}$ visualization to fire simulation. This will provide immediate benefits in translating real-life scenarios into computer models, and converting the results of those computer models into easily understood visualizations of simulation conditions. In the longer term, this combination of technologies has great potential in firefighter training and performance-based standards for building design.

\section{Approach}

In the first year, FY 1995, emphasis was on bringing CFAST and WALKTHRU together so CFAST could run a simulation while basic elements such as fire size and interface height were visualized in the WALKTHRU environment. Although the two systems operate on conceptually similar models of the world (i.e., a "cell-and-portal"structure), the specifics of the implementations are sufficiently different that efforts for the first few years will involve running the two programs on parallel, but separate world models. WALKTHRU provides the front end for interaction with and visualization of the virtual world, and CFAST provides the engine by which environmental conditions in the virtual world are updated. Presently, an integrated system is working in which CFAST and WALKTHRU work side-by-side, on a pair of models which are input separately. This procedure involves, one CFAST model contains the fire scenario, one WALKTHRU model contains the detailed world geometry, and a "registration map" which allow the two programs to relate their models of the world. Most of the FY 1995 and FY 1996 work involves the creation of the simulation models. The world geometry required by CFAST is a subset of the viewing geometry required by the WALKTHRU, so it is possible to automate or help the user in creating CFAST geometry from a WALKTHRU model. 3D model generation is a difficult and insufficiently studied task; a large part of our research contribution will be to develop techniques by which usable $3 \mathrm{D}$ models of interesting environments can be generated either by hand, interactively, and/or by automated processing of CAD floor plan information. This work will allow rapid and accurate creation of the environmental geometry which is needed for any simulation or visualization to take place. Recent results include the Building Model Generator (BMG), which automatically generates a WALKTHRU model and its corresponding registration map from an AutoCAD floor plan. BMG corrects common modeling errors and generates a complete $3 \mathrm{D}$ model with little or no human intervention, reducing the time required to model a building by two to three orders of magnitude. The second major thrust involves generating and modifying fire simulation scenarios interactively from within the virtual environment, and visualizing the results of the simulation intuitively and interactively in the virtual environment. It is intended to set a fire by 
walking into a room within the WALKTHRU and select a wastebasket or chair to begin burning, rather than juggling numbers and codes in a text file. Furthermore, using the WALKTHRU environment, one can visualize quantities such as interface heights, temperature distributions, and species concentrations spatially in real-time, rather than as two-dimensional plots. Work is currently underway on creating a user interface to set the simulation parameters from within the $3 \mathrm{D}$ virtual environment (location of the fire, environmental conditions during the simulation, and so on). The FY 1995 work resulted in the ability to take a normal CFAST fire scenario and visualize the fire size, plume, and interface heights within the environment in real time. This work continues into FY 1996. Techniques for visualizing the other spatial quantities that result from the simulation in the $3 \mathrm{D}$ environment will be developed. Once these techniques are developed, the user will be able quickly and easily to create a complete, highly accurate geometric model of an architectural environment. From within the model, they can set up simulations interactively, and watch the results unfold spatially and intuitively in real time.

\section{Recent Results}

Lewis, Richard W., Generating Three-Dimensional Building Models from Two-Dimensional Architectural Plans, Masters' Thesis, University of California, Berkeley, Berkeley, CA, in press.

\section{Enhanced PC-based Building Fire Assessment System}

Principal Investigator

Edward K Budnick, P.E.

Hughes Associates, Inc.

410-737-8677

\section{Sponsors}

National Institute of Standards and Technology

U.S. General Services Administrative

Public Building Services

Office of Real Property Management

\section{Objective}

To develop a PC-based version of the current Fire Safety Evaluation System for office occupancies and integrate selected quantitative methods that will enable engineers to perform detailed fire hazard, risk equivalency, and cost optimization analyses for existing or planned buildings.

\section{Problem}

The need to accurately measure fire risk impact and estimate costs of different fire safety strategies on levels of fire safety in individual buildings is essential to achievement of an overall building risk management philosophy. Limitations in current prescriptive requirements represent significant inhibitions to needed design flexibility and general usefulness. There is a growing need among building owners, local code authorities and design engineers about the availability of quantitative methods to evaluate building fire safety within a broad, risk-based context which permits examination of "equivalent" alternatives.

\section{Approach}

This effort is being conducted in three phases. Phase I was completed in FY 1995 and involved development of a PC-based version of the NFPA-FSES for business occupancies. This program was developed using the Microsoft Visual $\mathrm{C} / \mathrm{C}++$ Windows development platform and is fully compatible with the normal Windows environment. It contains explanatory text to assist the user in selecting the correct parameter conditions and uses visual prompts 
to guide the user through the FSES method.

The program contains four modules of encapsulated code, each with specific tasks.

The Applications Module is the main program. This module controls the individual performance elements, including calculations, output, and interface parameters.

The Main Window Module is the outer framework of the Windows-based program. It is analogous to the Program Manager in Microsoft Windows and allows the user access to file input/output features, print features, window controls, and program help.

The Document Module is the storage medium for user input. This module is invisible to the user. The document module contains all of the scoring functions, filters that check if data are complete and accurate, and all of the code for storing data to a file.

The View Module is the interface, used for entering data and selecting score categories. Currently, there are 15 elements in the View Module. The current version of the program has been validated on a limited basis against the FSES method in NFPA $101 \mathrm{~A}$ and can be used as an interim method for conducting FSES evaluations in office buildings.

Phase II was started in FY 1996 (completed in FY 1997) and is directed at the research to enhance the methodology to allow quantitative comparison of design options. This includes integration of appropriate elements of currently available engineering calculations (e.g., Hazard, FPETOOL, etc.) and development of algorithms to assess reliability.

Phase III will attempt to integrate the results and methods developed in the initial phases into a computerized risk management program.

\section{Recent Results}

(Cite Author(s)), Fire Safety Evaluation System (FSES) for Business Occupancies Software (Version 1.0 for Windows (B) User's Manual, NIST-GCR96-692, National Institute of Standards and Technology, March 1996.

\section{Accelerating the Application of Advanced Fire Safety Design Methods in the United States}

\author{
Principal Investigator \\ David A. Lucht, P.E. \\ Professor and Director \\ Center for Fire Safety Studies \\ Worcester Polytechnic Institute \\ 508-831-5593
}

\section{Sponsor}

National Institute of Standards and Technology

\section{Objective}

To accelerate the flow of modern fire protection engineering calculation methods into day-to-day practice. To increase knowledge and understanding of advanced fire safety design methods through development of educatior al packages targeted to fire safety design professionals and regulatory authorities.

\section{Problem}

Research of the past three decades is bearing fruit as new approaches to predicting fire performance through advanced calculation methods. For a host of reasons, many of these calculation methods are not yet enjoying widespread use among fire safety design professionals and regulatory authorities. One barrier to increase use is simply a lack of knowledge.

\section{Approach}

The initial effort focused on a calculation method that has become well established through peer review and consensus within the professional engineering community. The American Society of Civil Engineers (ASCE) Calculation Method for Structural Design for Fire Conditions has been selected for this initial effort. This methodology is in the final stages of being processed through the ASCE consensus process under ANSI protocol. The ASCE Committee on Special Design Issues: Fire Protection is in the process of preparing a design manual for this new ASCE document. Work is closely coordinated with this ASCE committee in the development of the manual and an accompanying educational package. 
During this first year the first draft of the manual will be completed, the target audience will be defined and specifications for the educational package will be completed. Initial progress has been delayed pending outcomes from the ASCE consensus process but work is moving forward in defining the audience and outlining the educational package.

\section{Recent Results}

New project

\section{Evaluation of Alternative Methods for Fire Rating Structural Elements}

\author{
Principal Investigator \\ Robert Dexter, Ph.D. \\ Senior Research Engineer \\ Center for Advanced Technology for Large \\ Structural Systems \\ Lehigh University \\ 610-758-6108
}

Sponsor

National Institute of Standards and Technology

\section{Objective}

The objective is to evaluate current computational methods for the assessing the integrity of structures exposed to fire and to develop practical tools for analysis in support of economical fire-safe construction.

\section{Problem}

The current U.S. Standard is ASTM E1 19, but this document has several disadvantages. ASTM E119 allows for two rating options, furnace testing with a simultaneous load, and, furnace testing without a simultaneous load. The United States does not have furnace test facilities capable of carrying out a column furnace test under load. Consequently, all ASTM E1 19 column ratings determined in the United States follow the no-load option.

Because of the inherently greater conservatism of the limiting-temperature criteria, ratings for the same section which redetermined from testing under load are expected to be somewhat larger than those determined by the limiting-temperature criteria in the no-load furnace tests. For a variety of reasons, structural elements in buildings are not usually loaded to anywhere near their maximum allowable limits. However, despite the expected level to which individual elements are actually loaded, present building codes would typically require like structural elements to have the same ASTM E1 19 rating. The structural responses of the individual elements are dependent on their end constraints, which: 1) are strongly coupled to the response of the overall structure; and, 2) can have major impact 
on element load-bearing capability. Clearly, computational thermal and structural analyses can provide a means of addressing and resolving these issues.

\section{Approach}

The approach is to evaluate computational methods for advancing accepted U.S. methods

of evaluating the fire performance of structures. This 2-year level of effort will review the range of possible approaches, from simple calculations to sophisticated numerical simulation, and gain competence with and apply at least one computer program. As for numerical simulations, it is planned to acquire and install at ATLAS, the specialized computer program SAFIR, developed by J-M. Franssen of the University of Leige, for simulating the thermal and structural response of fire-exposed structures. Dr. Franssen will train the principal investigator and a graduate research assistant and provide support for SAFIR. In addition, the capabilities and shortcomings of using more widely available and user-friendly computer programs such as ABAQUS will also be evaluated. Initial applications of SAFIR will involve simulating the response of structural elements as they are subjected to ASTM E1 19 furnace fire tests. This study will include simulations of loaded columns and/or composite floors label elements, previously tested under furnace conditions, and comparisons between computed and measured results. The objective of the initial SAFIR effort is to study the feasibility of using a computational approach to determine ASTM E1 19 ratings as a possible alternative to full furnace testing. Here, feasibility includes consideration of the practical issues of 1) instilling confidence in the U.S. building-code-making and -using community that a calculation-based ASTM E1 19-type method of determining fire resistance is a reliable alternative to the recognized ASTM E119 furnace test method, and 2) developing a plan on how to develop such an alternative to the point that it is recognized and adopted. At the conclusion of this 2-year program, the scope of the work necessary to complete the development of an advanced, calculation-based, fire-resistance rating system for use in United States building codes will be clearly defined. This future work will include consideration of:
1) the expanded use of thermal and structural analysis simulations to include evaluation of the fire performance of structural elements to fire environments other than the ASTM E1 19 standard fire, i.e., to real fire environments; and, 2) evaluation of the fire performance of entire structures, e.g., steel-framed buildings with composite floor slabs, first when exposed to the standard fire environment, and then to real fire environments.

\section{Recent Results}

New project 


\section{Computational Heat Conduction for Zone Fire Modeling}

\author{
Principal Investigator \\ William F. Moss \\ Department of Mathematical Sciences \\ Clemson University \\ 864-656-5225
}

\section{Sponsor}

National Institute of Standards and Technology

\section{Objective}

To develop, implement, and test a set of 1-D and 2-D heat conduction models suitable for incorporation into the NIST zone fire model CFAST. To develop the data structures necessary to code the transfer of heat between rooms through ceilings, floors, and walls. To develop a prototype zone fire model written in Fortran 90. Problem Implement the transfer of heat between adjoining rooms of a building in a zone fire model. Determine if vertical heat transfer in walls is significant by comparison of 1 and 2-D models.

\section{Problem}

In the NIST zone fire model CFAST, the four walls of the typical room are lumped together and divided into upper and lower sections by the smoke layer interface. A 1-D submodel for heat conduction into the upper and lower walls is implemented, but heat is not transferred between rooms. The radiation submodel used in CFAST computes configuration factors for the upper and lower walls which are functions of time because of the movement of the smoke layer interface.

\section{Approach}

Work performed resulted in the following recommendations. 1 . It is recommended that future versions of CFAST be written in Fortran 90. 2. Experimentation shows that the method used in CFAST of computing heat transfer through upper and lower walls to the outside is adequate. The finite difference method used in CFAST is a good choice .3. Experimentation indicates that the implicit, alternating direction method of Peaceman and Rachford (PR method) is a good choice for modeling 2-D heat conduction in walls. If the PR sweep in the $y$-direction is turned off, we have the PR x-only method which solves a set of $1-D$ heat conduction problems through the wall to approximate the $2-\mathrm{D}$ wall temperature profile. Using a simple logical switch, the PR method can be converted to the PR $\mathrm{x}$-only method soon subroutine can handle both models. The PR method is very efficient. The operation count is cut in half by switching to the PR x-only method. For thin walls, experimentation shows that there is no apparent difference between the outputs of the PR method and the PR x-only method. The PR x-only method can be used for thin walls. Being able to switch to the PR method, for thicker walls is an important feature of this approach. The current method of computing configuration factors for upper and lower wall surfaces that change with time is not computationally efficient. Also, the configuration factors associated with upper walls are indeterminate when the layer height is at the ceiling. A better approach would be to divide the wall into horizontal strips of equal height and then to compute and store configuration factors for all rooms before the simulation begins. In creating a simple radiation code of this type, the author gained an appreciation for the difficulties of this task. Radiation modeling is both programming intensive and computationally intensive. If a radiation model does not conserve energy, especially at low fire power, the simulation can be adversely affected with respect to both accuracy and run time. The differential-algebraic equation solver used in the simulation can be thought of as a mass and energy conservation engine that adjusts the solution variables at each time step by an iterative procedure so that mass and energy are conserved. Radiation models, especially poor ones, lead to slow convergence of the iterative method and increased computation times.

\section{Recent Results}

Moss, W.F., Computational Heat Transfer for Zone Fire Modeling, Final Report: NIST Grant No. 60NANB2D1281, Covering the Period June 1, 1994 to March 31, 1996, Clemson University, 1996. 


\section{Flow Through Vents in a Compartment Fire}

\author{
Principal Investigator \\ Yogesh Jaluria \\ Department of Mechanical and Aerospace \\ Engineering \\ Rutgers - The State University of New Jersey \\ 908-445-3652
}

\section{Sponsor}

National Institute of Standards and Technology

\section{Objective}

To carry out a detailed experimental study on the flow of smoke and hot gases in open vertical shafts, such as elevator shafts, focusing on the effects of ventilation, aspect ratio and operating conditions.

\section{Problem}

The flow in open vertical shafts is important to accurately model fire-induced flows in multi-leveled buildings since a considerable amount of transport of the combustion products occurs due to the shaft. The importance of the flow in vertical shafts has been demonstrated clearly in several recent fires in tall buildings, where the spread of the fire was strongly influenced by the presence of the shaft. Therefore, this transport mechanism must be included in the model for predicting the spread of smoke, hot gases and other combustion products, particularly toxic ingredients, at different levels in the building. Vertical shafts with large height to width ratio are of particular relevance to typical multi-storied buildings. In most cases, some form of ventilation, including forced ventilation, exists in these shafts. Forced ventilation may also be used to reduce the spread of toxicants in the building, allowing greater time for evacuation of the building.

\section{Approach}

An experimental system has been designed and fabricated for study of the flow in two different shafts, of height to width ratio (aspect ratio) of 3 and 10. Heated air is injected into the shaft at a lower opening and the downstream flow and temperature field are monitored by means of hot-wire anemometers and thermocouples. Visualization with injected smoke is also used for a qualitative view of the entire flow field. The conditions at the outlet are measured to determine the effects of entrainment into the flow, heat transfer to the walls and ventilation. Wide ranges of the physical variables in the problem are considered so that typical flows due to fire in multi-leveled buildings with vertical open shafts can be simulated. For the smaller aspect ratio shaft, the operating conditions were varied so that the transport processes range from high buoyancy levels, for which the entering flow was found to stay close to the vertical wall of the shaft, to much lower levels, for which the flow was found to spread outward with substantial entrainment and consequent dilution. With increasing inlet temperature, the buoyancy effect is increased, resulting in higher vertical velocities and shorter time to reach the top opening, as measured by optical monitoring of smoke. The time taken by the flow for traversing the distance between the two openings is an important quantity since it indicates the speed with which smoke and other combustion products reach into the higher levels of the building. The effect of the inlet flow rate is found to be relatively small, with a larger flow rate resulting in shorter time. The temperature at the outlet depends on heat transfer to the walls as well as on the flow velocity. These two effects oppose each other and the resulting ratio of the outlet temperature difference to the inlet temperature difference does not show a monotonic variation with an increase in inlet temperature. Detailed measurements on the velocity and temperature fields have also been taken. The different flow regimes that arise as the shaft aspect ratio, ventilation, and inlet conditions are varied are investigated. The results obtained on the unvented small aspect ratio shaft indicate that the velocity and the temperature are horizontally nonuniform in the shaft. A wall plume is generated which conveys the hot fluid rapidly along the shaft wall from the inlet to the outlet. A slow moving recirculating flow arises away from this wall, with this flow affecting the heat transfer and flow in the wall plume. This also affects the entrainment into the flow, decay of the temperature field and rate of downstream movement. Therefore, horizontal uniformity 
can not be assumed in this case.

The wall plume has to be modeled, considering the entrainment into this boundary layer flow and the interaction with the recirculating flow. The flow was found to rise to the closed top and generate a stratified region, eventually flowing out of the upper opening. When the side vent is opened, the entrainment was found to increase slightly. However, the mixing near the inlet increased substantially. This results in a lowering of the temperature level in the shaft. The effect of opening the top of the shaft had a much more substantial effect on the flow and heat transfer in the shaft. Very little flow was found to emerge from the top opening, as expected, and the temperature levels were found to be much lower and the velocity levels to be much higher. A detailed experimental study of the flow in the large aspect ratio vertical shaft (aspect ratio of 10 ) with and without ventilation has also been carried out. Again, wide ranges of the inlet temperature and flow rate were considered.

The discharge velocity of the hot gases or smoke was generally kept small to simulate the typical circumstance in building fires. The measurements included mass flow rates at the inlet and outlet channels, downstream decay of the temperature level, rate of smoke movement, and temperature and velocity fields in the shaft. It was found that the temperature distribution is fairly uniform across a horizontal plane in the shaft, making it possible to develop a simpler one-dimensional model. Thus, the flow is fairly well mixed as it moves downstream and the wall plume-recirculating flow structure is not observed. The effects of ventilation by opening the side vent or the shaft top were similar to those observed earlier. The results obtained were also compared with those from the first part of the study on small aspect ratio shafts. All the results are obtained in dimensionless terms to facilitate their use in the modeling of building fire situations. The ranges of the governing parameters are chosen to cover typical fires in multi-leveled buildings.

This effort is expected to lead to a better understanding of the flow in vertical shafts and to yield quantitative inputs which may be used for modeling fires in multi-leveled buildings. The important physical aspects to be investigated include ambient fluid entrainment, dilution, speed of smoke spread and effects of forced ventilation.

\section{Recent Results}

Kapoor, K and Jaluria, Y., "Flow and Heat Transfer Due to a Buoyant Ceiling Jet Turning Downward at a Corner," ASME J. Heat Transfer, Vol. 118 , pp. 38-46, 1996.

Mercier, G.P., Fire-Induced Flow of Smoke and Hot Gases in Vertical Shafts, M.S. Thesis, Rutgers

Univ., New Brunswick, NJ, Jan. 1996.

Jaluria, Y., Chiu, W.K.S. and Lee, S.H.-K, "Flow of Smoke and Hot Gases Across Horizontal Vents in Room Fires," Combust. Sci. Tech., Vol. 110-111, pp. 197-208, 1995.

G.P. Mercier and Jaluria, Y., "Fire-Induced Flow of Smoke and Hot Gases in Open Vertical Shafts," Symp. Thermal Sci. Engg., Berkeley, CA, pp. 261-268, 1995.

Abib, A. and Jaluria, Y., "Turbulent Penetrative and Recirculating Flow in a Compartment Fire," ASME J. Heat Transfer, Vol. 1 17, pp. 927-935, 1995. 


\section{Flows in Vertical Shafts}

Principal Investigator

Edward E. Zukoski

California Institute of Technology

818-395-4785

\section{Sponsor}

National Institute of Standards and Technology

\section{Objective}

To provide the information required for the development of computer-based models for flows in vertical shafts and passages during a fire within a building.

\section{Problem}

The research program in progress at Caltech involves the development of a description of transient flow of buoyant gas in a vertical shaft. Flows of this type are a primary means of transport of hot, toxic gas through a multistoried structure and the description of this process is not yet available for use in computerbased fire codes. Examples are the flow of hot gas up ventilation and elevator shafts or stairwells when the stack effect is absent or weak.

\section{Approach}

The basic study involves an investigation of the flow in a vertical shaft filled with cool air before the start of the experiment and has walls that are at room temperature. A typical experiment starts when the bottom of the shaft is suddenly opened and exposed to a large reservoir filled with hot toxic gas. A turbulent mixing process is produced because high density air is placed above the lower density hot gas. The subsequent penetration of the hot gas and toxic species into the shaft by means of the turbulent mixing process and the better known stack effect is the subject of this study.

Experiments are being carried out in a shaft that is $0.25 \mathrm{~m}$ square and $2.4 \mathrm{~m}$ high. The shaft is open at the bottom and the walls, composed of $2.5 \mathrm{~cm}$ thick aluminum plates, remain close to room temperature during a typical experiment. Heat transfer to the walls of the shaft affects both the stack and turbulent mixing processes. The effect on the shaft flows of openings at various positions is also being investigated under conditions in which both processes are important.

This investigation is an ongoing program that will take several years to complete. Initial studies of the temperature field and heat transfer in a closed shaft due to the turbulent mixing process have been completed, and studies of the motion of toxic species in the shaft and flows in which both stack and turbulent mixing processes are important have begun.

\section{Recent Results}

Initial results show that the long time-averaged spatial distribution of temperature and wall heat transfer rates reach a steady state immediately after the hot gas first reaches the top of the shaft and that the presence of heat transfer has a dramatic impact on the density distribution in the shaft. 



\section{FIRE SAFETY ENGINEERING}

DIVISION

LARGE FIRE RESEARCH 

Fire Flows

\section{Principal Investigator}

Howard R Baum

Fire Safety Engineering Division

301-975-6668

\section{Sponsor}

National Institute of Standards and Technology

\section{Objective}

To develop a fundamental understanding of the mechanisms, which control the generation and transport of heat and smoke in fires and to develop a predictive capability that will allow the computer simulation of fires associated with constructed facilities to be based on the underlying physical principles.

\section{Problem}

The interaction of fires with the physical environment in commercial, industrial, residential, and transportation facilities has been a major research topic for BFRL and its international counterparts since its inception. The dominant problem of heat and smoke movement in these facilities is of interest to their builders, owners, operators, regulators, and insurers. Small scale combustion processes are the study of diffusion flames in a turbulent flow environment and as such are of interest to the general combustion community. Simulations performed to date have helped the petrochemical industries, the State of Alaska, and the U.S. Minerals Management Service.

\section{Approach}

During FY 1996, BFRL will continue to perform detailed studies of individual phenomena of transport, mixing, diffusion, radiation, and reaction processes occurring at widely different length and time scales. Theoretical and computational techniques applied to the study of these phenomena are carried out in a way that permits them to be assessed individually and later combined into overall simulations of problems of interest.

\section{Recent Results}

McGrattan, KB., Baum, H.R, and Rehm, RG., "Numerical Simulations of Smoke Plumes from
Large Oil Fires," Atmospheric Environment, Vol. 6, (In press), 1996.

Baum, H.R, McGrattan, KB., and Rehm, RG., "Large Eddy Simulations of Smoke Movement in Three Dimensions," Conference proceedings of the Seventh International Interflam

Conference, Interscience Communications, London pp. 189-198, 1996.

Baum, H.R, McGrattan, KB., and Rehm, RG., "Simulation of Smoke Plums from Large Pool Fires," 25th Symposium on Combustion, The Combustion Institute, Pittsburgh, PA, pp. 14631469, 1994. 


\section{Large Eddy Simulation (LES) Verification}

\author{
Principal Investigator \\ Kevin McGrattan \\ Fire Safety Engineering Division \\ 301-975-2712
}

\section{Sponsor}

National Institute of Standards and Technology

\section{Objective}

To verify the LES field model applied to the prediction of ceiling temperatures and sprinkler/draft curtain interactions.

\section{Problem}

The high resolution potential of the LES field model has been difficult to verify, especially when applied to large spaces and complicated flow patterns such as those introduced by automatic sprinklers. Current measurement technologies are limited by spatial resolution and advanced measurement techniques are generally limited to laboratory scale experiments. Despite the limitations, verification is a critical aspect to the development and acceptance of the LES model.

\section{Approach}

During FY 1996, BFRL will compare LES predictions with the results of experiments conducted at FMRC which addressed the issue of sprinklers and draft curtains. Funding for large scale experiments will be sought from the NFPA Research Foundation. Hot nontoxic smoke has been used in several countries to conduct acceptance tests for smoke control systems. This technique will be examined to determine its applicability to LES verification.

Infrared thermography will be used to obtain verification data. An infrared thermography system will determine the temperature of the walls or ceiling in a fire compartment. An infrared camera will be used to measure the temperature of the unexposed side of a thin metal ceiling in a small scale experiment. The measurements will be compared with the predictions of the LES model including new capabilities for heat transfer being developed in the Fire Flows project. Although the infrared measurements will not precisely be the wall temperatures, they can be used to determine the relative temperature field over a large area. The results of the small scale experiments, will be used to evaluate the potential for using this technique in larger experiments or small scale experiments with complex ceiling structures.

Sprinkler hardware from tests conducted by FMRC was characterized for flow and air entrainment in tests carried out in an aircraft hanger in Richmond, VA. The measurements have been used to create a realistic simulation of the sprinkler spray for use in the LES model.

\section{Recent Results}

New project 


\section{Smoke Plume Trajectory From in situ Burning of Crude Oil in Alaska .. Phase II}

\section{Principal Investigator \\ Kevin McGrattan}

Fire Safety Engineering Division

301-975-2712

\section{Sponsors}

Alaska Department of Environmental

Conservation

Division of Spill Prevention and Response

Environmental Management Consultants, Alaska, Inc.

\section{Objective}

To provide information about the expected trajectory of smoke plumes from the use of in situ burning as a response method for oil spills in Alaska.

\section{Problem}

There is need to determine the downwind concentrations of smoke particulate from the in situ burning of oil on water with emphasis on multiple burns and on complex terrains of Alaska.

\section{Approach}

During FY 1996, BFRL will extend the application of the LES model of a smoke plume trajectory to account for multiple burns and complex terrain. Specific areas of concern in Alaska will be used for simulations. In addition, BFRL will provide technical support to EMCON in its bid to secure EPA approval for the application of the LES model to Alaska. BFRL also will install, in Alaska, a version of the LES model to be used by EMCON and ADEC personnel.

\section{Recent Results}

McGrattan, KB., Walton, W., Putorti, A.D., Twilley, W.H., McElroy, J., and Evans, D.D., Smoke Plume Trajectory from In-Situ Burning of Crude Oil in Alaska, NISTIR 5764, National Institute of Standards and Technology, November 1995.

\section{In-situ Burning of Oil Spills}

Principal Investigator

William D. Walton

Fire Safety Engineering Division

301-975-6872

\section{Sponsor}

Department of the Interior

Minerals Management Service

Technology Assessment and Research Branch

\section{Objective}

To determine conditions where in-situ burning can be used effectively as a response method to accidental spills of crude oil on land and on water.

\section{Problem}

In support of other agency objectives to carry out burning as a response method to oil spills, predictions are needed to assess the impact of the smoke plume produced by burning on downwind locations. BFRL's Large Eddy Simulation (LES) model continues to gain acceptance for use in planning in-situ burns although there has been limited verification. There is a need for predictions that take into account the effect of local terrain features such as mountains. Further, the spill response planning community has shown a strong interest in using the LES model as a planning tool.

One of the most significant remaining obstacles to the acceptance of burning as a spill response technique is the lack of a standard method for evaluating a fire resistant oil spill containment boom. To develop a test method, knowledge of the thermal exposure conditions and physical stresses that the boom is expected to be exposed to, must be determined.

\section{Approach}

During FY 1996, BFRL will continue to verify its LES model using the LIDAR smoke plume data collected by SRI but not yet available in final form. Using the University of Oklahoma's Advanced Region Prediction System, local terrain effects will be added to the model. The feasibility of developing a PC version of the LES 
model will be examined, and if practical, a PC version developed.

To fully characterize the thermal exposure conditions on a fire resistant boom, a series of experiments will be performed to measure the exposure of the boom during a characteristic spill fire. These burns will examine the effect of spill size and fuel type. If a reduced smoke fuel or burning arrangement can be found which produces the same thermal exposure as a real spill, then one the obstacles to conducting future experiments at facilities such as OHMSETT would be eliminated.

\section{Recent Results}

McGrattan, KB., Walton, W., Putorti, A.D., Twilley, W.H., McElroy, J., and Evans, D.D., "Smoke Plume Trajectory from In-Situ Burning of Crude Oil in Alaska - Field Experiments," Proceedings of the 18th Arctic and Marine Oil Spill Program (AMOP) Technical Seminar, Edmonton, Alberta, 14-16 June 1995, pp. 901913, 1995.

Walton, W., Twilley, W.H., Putorti, A.D., and Hiltabrand, RR, "Smoke Measurements Using and Advanced Helicopter Transported Sampling Package with Radio Telemetry," Proceedings of the 18th Arctic and Marine Oil Spill Program (AMOP) Technical Seminar, Edmonton, Alberta, 14-16 June 1995, pp. 1053-1074, 1995.

\section{Demonstration of Water-Based Fire Fighting Agents}

\author{
Principal Investigator \\ Daniel Madrzykowski \\ Fire Safety Engineering Division \\ 301-975-6677
}

\section{Sponsor}

Federal Emergency Management Agency

U.S. Fire Administration

\section{Objective}

To develop methods for demonstrating biodegradable, environmentally safe, nontoxic fire suppression liquids which are effective on Class A, B and many D fires.

\section{Problem}

Water-based fire fighting agents have been used for many years to enhance the fire fighting capabilities of ordinary water. The most widely used of the water-based agents are the foams for use on Class B fires. Agents designed primarily for Class A fires were used extensively with wildland fires. More recently, these agents were promoted for use on a wider range of Class $\mathrm{A}$ and sometimes Class B and D fires. These agents are frequently advertised as more effective than plain water while being environmentally safe. Sometimes they also are claimed to reduce the quantity and toxicity of smoke.

There are several commercially available waterbased fire suppression agents designed primarily for Class A fires. Generically these agents can be classified as surfactants which reduce the surface tension of water, potentially modifying the fire fighting capabilities. There are standards for assessing these agents, however most of the criteria do not address the fire fighting (protection/suppression) capabilities of the agent. This is particularly true for Class $\mathrm{A}$ and Class D fires. An evaluation protocol is needed to measure the fire fighting capability of an agent and to relate its performance to plain water or another agent in a given situation. This would enable the fire protection community to select the most cost effective fire suppression agent(s) to fit their specific needs, 
thus optimizing use of their resources.

The use of water-based fire fighting agents raises the question of potential health and environmental effects. First is the exposure of fire fighters to the agent itself and the products of combustion produced when using the agent. Second is the impact as agent run off enters the environment. These issues have generally been addressed with standard toxicity and environmental impact tests, although a complete series of these tests has not been performed even for the most commonly used agents.

\section{Approach}

During FY 1996, BFRL will perform three tasks:

1. Collect information on agents which are considered by their manufacturer to be biodegradable, environmentally safe, nontoxic fire suppression liquids which are effective on Class A, B and many D class.

2. Develop methods and assess the biodegradability, environmental safety, toxicity and physical properties of some water-based fire fighting agents.

3. Develop methods and demonstrate the fire fighting effectiveness of several water-based fire fighting agents for Class A, B and D fires.

\section{Recent Results}

New project

\section{Demonstration of New Residential Suppression System Technologies}

\author{
Principal Investigator \\ Daniel Madrzykowski \\ Fire Safety Engineering Division \\ 301-975-6677
}

\section{Sponsor}

Federal Emergency Management Agency

U.S. Fire Administration

\section{Objective}

To develop a demonstration program based on technical information for system design and full scale fire suppression tests for Limited Area Dwelling Sprinkler Systems and Residential Water Mist Fire Suppression System technologies.

\section{Problem}

The United States Fire Administration (USFA) has been involved in the research and development of the Limited Area Dwelling Sprinkler Systems (LADSS) and Residential Water Mist Fire Suppression System (RWMFSS) technologies. Over the last three years, USFA sponsored research on these systems in developing residential sprinkler systems with minimized water requirements. This research has been conducted by Factory Mutual Research Corporation, Hughes Associates Inc., the National Institute of Standards and Technology, and Underwriters Laboratories. The USFA would like to develop a demonstration program to continue their transfer of information on these technologies to the fire community.

\section{Approach}

During FY 1996, BFRL will perform two tasks:

1. Develop a demonstration program based on technical information for system design and full-scale fire suppression tests for LADSS and RMFSS technologies.

2. Conduct a meeting at NIST with speakers representing USFA research programs and to conduct full scale fire suppression tests to demonstrate the technologies. 
Recent Results

Conducted demonstration Program/Meetings at BFRL, March 12, 1996.
Evaluation of Water Mist System

Technology for Light Hazard

Occupancy Applications

\author{
Principal Investigator \\ Daniel Madrzykowski \\ Fire Safety Engineering Division \\ 301-975-6677
}

Sponsor

Federal Emergency Management Agency

U.S. Fire Administration

\section{Objective}

To evaluate the performance of commercially available water mist system technologies compared with a NFPA 13/13D system for light hazard/residential occupancies.

\section{Problem}

Several types of water mist systems have been commercially developed for use as fire suppression systems. The potential benefits of a water mist system include quick-response, smaller water supply requirements concerning a sprinkler system and less collateral damage from excess water. These potential benefits make water mist systems attractive as an alternative for occupancies that currently would require a sprinkler system. If effective, water mist systems could be used in light hazard occupancies such as offices and residences.

Currently, an installation standard is being developed for water mist systems by the National Fire Protection Association and the testing laboratories have proposed requirements for listing of water mist system equipment for specific applications. However, there is a gap in the knowledge base regarding the applicability of the systems to different occupancies. This study investigates the applicability of a low pressure, high momentum water mist system to light hazard occupancies.

\section{Approach}

During FY 1996, BFRL will conduct large scale compartment fire experiments with a commercially available low pressure, high momentum water mist system technology. A limited number of fuel packages representative 
of light hazard occupancies such as offices and residences will be selected in consultation with the U.S. Fire Administration (USFA). Replicate experiments then will be conducted with sprinkler systems meeting the criteria of NFPA 13/13D for comparison.

\section{Recent Results}

New project

\section{Office Building Fire Research Program}

\section{Principal Investigator}

Daniel Madrzykowski

Fire Safety Engineering Division 301-975-6677

\section{Sponsor}

General Services Administration

Public Buildings Service

Office of Real Property Management and Safety

\section{Objective}

To quantify the impact of large fires on buildings and their occupants, and investigate the use of current technology/resources for mitigating the hazards.

\section{Problem}

Systems furniture or "work stations" have been identified as a source of large heat release rate fires in office buildings. Because of the wide spread use of systems furniture, the potential fire hazard in an open plan office environment needs to be quantified. The conditions in a long corridor (means of egress) adjoining the fire compartment needs to be determined. If the fire cannot be suppressed in the room of origin, the impact of sprinklers on the smoke flow in the corridor is needed. While the impact of sprinklers has been demonstrated in BFRL experiments, it has not been included in the corridor flow model, because the data base and zone modeling techniques were not sufficient to continue development of the corridor flow model including sprinkler effects.

\section{Approach}

During the past three years, BFRL has conducted experiments on examining smoke flow in a corridor and characterizing the heat release rates of open plan office fuel packages. Experiments also were conducted on mitigating the hazard of such fires.

During FY 1996, BFRL will conduct experiments to model the mitigation effects and efforts aimed at verifying FPEtool (BFRL's software used to evaluate hazards and fire protection strategies in GSA office buildings). 
Specifically the water spray from the sprinklers used in the large scale experiments will be quantified. This information will be used as input to the FIRE DEMAND model and as input for field modeling of the corridor experiments. Large scale experiments on the effects of obstructed ceiling flows on sprinkler activation will be conducted. Experimental results will be compared with the current capabilities of FPEtool.

\section{Recent Results}

Madrzykowski, D., "Office Work Station Heat Release Rate Study: Full Scale vs. Bench Scale," Interflam'96 Proceedings, Cambridge, England, March 1996.

Putorti, A.D., Belsinger, T.D., and Twilley, W.H., Determination of Water Spray Drop Size and velocity from a Low Pressure, High Momentum Water Mist Nozzle, NIST FR4000, National Institute of Standards and Technology, May 1995.

\section{Northridge Post-earthquake Fire Assessment}

Principal Investigator

William D. Walton

Fire Safety Engineering Division

301-975-6872

\section{Sponsor}

Federal Emergency Management Agency

United States Fire Administration

\section{Objective}

To examine the origin and spread of fires and evaluate the response of fire sprinkler systems following the Northridge earthquake.

\section{Problem}

The Northridge earthquake provides an opportunity to examine the causes of postearthquake fires, effectiveness of building systems in suppressing and containing the fires, damage to the fire protection infrastructure, management of fire department resources in response to the fires, and the recovery from fire damage following an earthquake. Fire sprinkler systems are one of the most widely used active fire protection systems in buildings. There is preliminary evidence that the Northridge earthquake resulted in damage to many sprinkler systems.

\section{Approach}

During FY 1996, BFRL will work with grantees and contractors to analyze the information concerning the causes, effects and response to fires following the Northridge earthquake. The project will take into account the complex interactions of events related to fire to determine the actions which would be effective in reducing the loss due to fire and maintaining adequate fire protection after an earthquake.

The principal tasks are:

1. Develop recommendations for retrofit and new construction to improve the reliability of fire sprinkler systems and reduce the recovery time.

2. Develop a methodology which can be used by fire inspectors to evaluate the condition of building active fire protection features following earthquakes. 
3. Demonstrate the effectiveness of a temporary emergency measure developed at NIST to reduce the spread of fire from the outside of a structure through windows to the interior of a structure.

\section{Recent Results}

Chung, R, M., Jason, N, H., Mohraz, B., Mower, F.W., and Walton, W.D., Eds., PostEarthquake Fire and Lifelines Workshop, Long Beach, CA January 1995 Proceedings, NIST SP 889, National Institute of Standards and Technology, August 1995.

\section{Modification of Fire Protection Requirements for NRC}

Principal Investigator

Robert S. Levine

Fire Safety Engineering Division

301-975-6671

Sponsor

U.S. Nuclear Regulatory Commission

Office of Nuclear Regulatory Research

\section{Objective}

To support the Nuclear Regulatory Commission (NRC) develop a new "rule" that will permit utilities to use performance-based methods to design fire protection provisions for nuclear power plants.

\section{Problem}

Current regulation is based on Appendix $\mathrm{R}$ of 10 CFR 50 which is entirely prescriptive.

\section{Approach}

During FY 1996, BFRL will review the status and problems of using, world wide, performance-based fire safety design and regulation in the built environment, and in nuclear power plants in France and Japan. Staff will meet with Brookhaven National Laboratory on probability reliability assessment techniques, which can be used to rate the importance of various intervention strategies. NRC prescriptive fire protection requirements will be compared with NFPAs and with other codes with a view toward reducing the cost of fire safety where a cost saving is justified. NRC will be provided technical support to help them better prepare documents.

\section{Recent Results}

Provided interim reports to sponsor. 


\section{Firefighter Thermal Exposure and the Performance of Protective Clothing in Thermal Environments}

\section{Principal Investigator}

James R. Lawson

Fire Safety Engineering Division

301-975-6676

\section{Sponsor}

National Institute of Standards and Technology

\section{Objective}

To determine the thermal performance of firefighters protective clothing by developing measurement equipment and techniques to evaluate and measure the thermal exposures experienced by firefighters while conducting fire suppression and rescue tasks and the performance of the firefighter's protective clothing.

\section{Problem}

Firefighters' protective clothing and equipment have experienced significant performance improvements over the last few decades; however, thousands of firefighters continue to be burned each year. The apparatus presently used to measure the performance of firefighter clothing can only subject the clothing to fire exposures created in the laboratory. No industrial standards for acceptable performance exist.

\section{Approach}

During FY 1996, BFRL will conduct a literature survey to identify applicable publications associated with protective clothing and equipment thermal measurements, firefighting operations, burn injuries and performance of protective clothing. Obtain information from the fire service and protective clothing equipment manufacturers to identify special needs of firefighters and industry. Data gathered during these studies will be used to understand the thermal environments better, firefighter activities and protective clothing, and equipment issues related to firefighter burn injuries. BFRL staff will construct a prototype portable instrument package for measuring the performance of firefighters protective clothing and equipment in various fire fighting environments. The prototype will be used to evaluate instruments and measurement techniques.

\section{Recent Results}

Lawson, J., Firefighter's Protective Clothing and Thermal Environments of Structural Fire Fighting, NISTIR 5804, National Institute of Standards and Technology, 1996. 


\section{Development of a Sizing Standard for Firefighter Protective Clothing}

\section{Principal Investigator}

James R. Lawson

Fire Safety Engineering Division

301-975-6676

\section{Sponsor}

Federal Emergency Management Agency

U.S. Fire Administration

\section{Objective}

To determine the critical factors of work/station garment size that affect the thermal protection and work dynamics of firefighters during fire suppression operations.

\section{Problem}

From recent changes in employment regulations in the fire service the range of firefighter body sizes has increased. The fit of firefighter work/station uniforms has become more critical as it relates to function with and without protective garments in fire suppression operations. Therefore, a need exists for standardizing the size of work/station uniforms to enhance safety and efficiency in fire fighting activities. In addition, emergency responders from FEMA have no standards that provide guidance on protective clothing or equipment required when they are called to investigate emergencies or disasters.

\section{Approach}

During FY 1996, BFRL will conduct a literature survey to identify applicable publications related with the fit and function of firefighter work/station uniforms and clothing and about equipment for use by FEMA personnel in an emergency or hazardous environments.

Researchers will obtain information from the fire services and apparel manufacturers to identify special needs of firefighters and the ability of industry to support the firefighter's needs. Also, data will be obtained from emergency managers and investigators to identify their needs for protective clothing and equipment. Staff will work with ASTM's Committee F-23 on Protective Clothing and national Fire Protection Association (NFPA) committees which produce protective clothing and equipment standards to develop and establish standards for firefighter work/station uniforms. The results will be published in a guide document on protective clothing and equipment for use by FEMA emergency managers, investigators and inspectors.

\section{Recent Results}

Developed a draft "Standard Practice for Body Measurements and Sizing of Fire and Rescue Services Uniforms and Other Thermal Hazard Protective Clothing." 


\section{Large Fire Research Facility}

Principal Investigator

Daniel Madrzykowski

Fire Safety Engineering Division

301-975-6677

\section{Sponsor}

National Institute of Standards and Technology

\section{Objective}

To perform large scale fire experiments and provide large fire measurement capabilities that meet the needs of industry and government customers.

\section{Problem}

Use of BFRL's large fire research facility must be scheduled for timely performance of experimental programs. Modernization of equipment and facilities is required to address issues of environmental and work place safety.

\section{Approach}

During FY 1996, BFRL will continue to provide daily management of the Large Fire Research Facility, including scheduling tests for timely execution along with the gradual modernization of equipment and facilities. Additional short term improvements will be developed and executed during the fiscal year, pending availability of funding. These plans will be developed with researchers to assure that newly and updated instruments fit the needs of the fire program and its customers. BFRL will develop methods to assure full compliance with Federal environmental requirements for large and small scale tests.

\section{Recent Results}

Completed a feasibility study for renovating the emissions control system and began renovations on the interior of the test bay. 
RECENT GRANTS - LARGE FIRE $\underline{\text { RESEARCH }}$

\section{Computational Modeling of Large Fire Plumes}

\author{
Principal Investigator \\ Professor A.F. Ghoniem \\ Department of Mechanical Engineering \\ Massachusetts Institute of Technology
}

\section{Sponsor \\ National Institute of Standards and Technology}

\section{Objective}

To develop a computational based large fire plume model in which buoyant fluid mechanics, combustion, radiative transport, and their mutual interactions are all fundamentally represented using physically sound

approximations, and well resolved spatially and temporally.

\section{Problem}

Simulation of fire plumes yielding burning rates, pollutant formation rates and the radiation field is necessary as a tool for the assessment of the environmental impact of large fires.

Computational modeling of these fires is complicated by the presence of several complex physical processes, including turbulence, transport and chemistry. The wide range of physical scales present in these processes complicates their numerical modeling even further. The overall dynamics is essentially unsteady and thus should be the model.

Conventional numerical approaches could be very expensive since the domains of these fires are often extremely large.

\section{Approach}

To overcome the difficulties described above, we are developing a computational model of fire plumes based a Lagrangian simulation of all significant physical processes involved. The vortex method is used to obtain solutions of the Navier-Stokes equations, including the buoyancy terms and the baroclinic terms, using computational elements distributed along the layer separating the plume (fuel and products) and the surrounding (air and products), in the turbulent regime. The mixing and combustion processes are simulated using the transport element method in which only species concentration and temperature gradients are computed, and only where vortex elements exist. The fast chemistry assumption is used in the computation of the burning rate, temperature and major species distribution. The concentration of the computational elements within the narrow zone of plume boundaries endows the approach with the necessary efficiency.

During FY 1996, work is underway on improving the code performance by incorporating analytical expressions for the fields (velocity, temperature, density and scalar concentrations) instead of evaluating them from elliptic integral expressions. We used the new code to simulate fire plumes raging over evaporating liquid fuel pools and used the results to explain the origin of puffing in and the similarity between fire plume and isothermal plume puffing. We also have improved the discreditization accuracy and incorporated a more accurate diffusion approach, both are extremely important in evaluating the burning rate.

We are currently devising a gas radiation algorithm to incorporate this all important heat transfer mode which, in the presence of soot, is regarded as the primary heating mechanism for the pool. The model's computational efficiency, achieved by using Lagrangian schemes to simulate each of these processes, should allow practical simulations to be performed on an advanced engineering workstation.

\section{Recent Results}

Ghoniem, A. F., Lakkis, I., and Soteriou, M., "Numerical Simulation of the Dynamics of Large Fire Plumes and the Phenomenon of Puffing," accepted for presentation at the Twenty-sixth Symposium (International) on Combustion, Napoli, Italy, July 28-August 2, 1996. 


\section{A Study of Two Phase High Liquid Loading Jet Fires}

\author{
Principal Investigator \\ Jay P. Gore \\ Purdue University \\ 317-494-1452
}

\section{Sponsor \\ National Institute of Standards and Technology}

\section{Objective}

To study visible flame lengths, radiation and pollutant emission properties of high liquid loading jet fires, and their gas fired analogs.

\section{Problem}

Two phase jet fires result from effective atomization of a liquid fuel by small quantities of trapped gases. The oil well fires in Kuwait are the most prominent example. The visible flame lengths of such fires increase with increasing heat release rates even in the momentum dominated regime. This increase is contrary to existing knowledge. Correlations for flame length, radiative fractions and pollutant emission indices for such fires are also not available.

\section{Approach}

An effervescent atomizer burner simulates the physical processes that occur in accidental fires involving high liquid loading two phase jets.

We built a small burner for operation in the 10 $\mathrm{kW}$ to $60 \mathrm{~kW}$ firing rate range and a large burner for operation in the $30 \mathrm{~kW}$ to $200 \mathrm{~kW}$ firing rate range. We have measured: radiative heat loss fractions, visible flame lengths, chemical flame lengths, and pollutant emission indices for several spray fires stabilized on these burners. Toluene, heptane, methanol and crude oil were the liquid fuels. The atomizing gas was methane. A hydrogen pilot flame stabilized the spray fires.

We defined the chemical flame length of the spray fires based on the existence of minimal hydrocarbon concentration $\left(\mathrm{CH}_{4}<0.05 \%\right)$ along the axis. We defined the chemical flame length of acetylene fires based on lower than $0.05 \% \mathrm{C}_{2} \mathrm{H}_{2}$ concentration along the axis. We measured: the gas species concentrations by sampling with a water-cooled probe and analyzing with a gas chromatography; soot emission index using sampling on filters weighed on a microbalance; $\mathrm{CO}$ emission index using a gas filter correlation analyzer, and $\mathrm{NO}_{\mathrm{x}}$ emission index using a chemiluminescence analyzer.

Pollutant emission indices for soot, $\mathrm{CO}$ and $\mathrm{NO}_{x}$ varied significantly with the amount of atomizing methane.

Measurements of visible flame length for the large spray fires showed a continued increase with heat release rate. Analogous experiments with acetylene/air flames showed that such an increase occurred for luminous gaseous jet fires as well. Analyses of turbulent non-premixed gaseous jets show that stoichiometric mixing occurs at a fixed normalized height above the injector exit. Therefore, we focused attention on whether the visible flame length has a fixed relationship to the length required for turbulent mixing to complete combustion.

Measurements of the chemical flame length for both gas-fired and spray fires strongly support the prediction that turbulent mixing to complete combustion occurs at a fixed normalized height above the injector exit.

Thus soot processes such as radiative cooling to a black exhaust or oxidation to invisible gases are responsible for the changes in the visible flame length. These processes are now being studied in more detail.

\section{Recent Results}

Lawler A., Wade R, Sojka P. E. and Gore J. P., "Flame Length and Pollutant Emission Characteristics of Effervescent Atomizer/Burner Stabilized Jet Flames," Proceedings of the

Technical Meeting of the Central States Section of the Combustion Institute, 1996.

Wade R. and Gore J. P., "Visible and Chemical Flame Lengths of Acetylene/Air Jet Diffusion Flames," ibid. 


\section{Measurement of Flame Temperature Using IR Technique and Air-Entrainment Velocity Using PDPA}

\author{
Principal Investigator \\ Kozo Saito \\ Department of Mechanical Engineering \\ University of Kentucky \\ 606-257-1685
}

\section{Sponsor}

National Institute of Standards and Technology

\section{Objective}

To measure temperature profiles of large scale liquid pool fires using an infrared imaging temperature measurement technique (IRITM) and evaluate the accuracy of the IRITM data; measure the flow structure at the flame base for small scale pool fires using a laser sheet particle track technique; and conduct scaling analysis on these data.

\section{Problem}

The structure of large scale pool fires is of interest, because it is associated with radiant emission to the environment, formation of environmentally unacceptable combustion byproducts, flame blow-off by extinguishment agents, and other fire safety reasons.

\section{Approach}

Design small scale pool fire experiments and measure temperature profiles in these flames using IR technique and velocity profiles using a laser sheet particle track technique. With the collaboration of NIST and FRI in Japan, have completed IR temperature measurements for a $15 \mathrm{~m}$ diameter crude oil fire. This large scale fire data can be used as the reference to develop scaling correlations. Using a commercial CFD code made it possible to calculate the fire plume behavior and flow profiles near the flame base. The results will produce an improved understanding of the structure of large scale pool fires.

\section{Recent Results}

New project

\section{Fire Protection in Large Industrial Fires}

\author{
Principal Investigator \\ Marino diMarzo \\ Mechanical Engineering Department \\ University of Maryland \\ 301-405-5257
}

\section{Sponsor}

National Institute of Standards and Technology

\section{Objective}

To evaluate and optimize the performance of fire fighting agents used to protect structures and to prevent the spreading of fire in large industrial settings such as large petrochemical storage facilities.

\section{Problem}

The most common active methods to mitigate and contain large industrial fires in petrochemical storage facilities encompass water sprays and water films. These techniques require massive amounts of water due to the significant duration of these fires. The water runoffs are contaminated by the petrochemical spills and may constitute a significant environmental concern. An integrated approach to identify cost effective, environmentally safe agents and their application strategies is sought.

\section{Approach}

The specific goals of this research include: a) the evaluation of the active techniques currently used; b) the experimental and theoretical study of the interactions of the fire fighting agents with the fire environment; c) the extension of these findings to identify the optimal agents to be used; d) the identification of the most costeffective, environmentally safe application methodology; and e) the setting of guidelines for the design and performance prediction of preventive measures and fire mitigation strategies applicable to large industrial settings.

Protein foams exhibits characteristics which are of particular interest for these applications. They show: a) good expansion characteristics to provide significant volume of foam per unit volume of liquid; b) good performance about 
burnback resistance when exposed to fire; and c) low mobility which makes them ideal to coat vertical and underhung and horizontal surfaces. Fire trials, which test the overall performance of the foaming agents, are available. However, the data compare the various foams based on the time before ignition of the protected surface. Human factors and outdoor uncontrolled environmental conditions are common obstacles to the consistent repeatability of these kinds of tests. Nonetheless, the test procedures and product rating reflect these overall approach limitations.

The quantification of the foam performance when exposed to a controlled fire environment and the linkage of the foam behavior to its thermal and physical characteristics is the key to optimize the application strategy and the foaming agent property requirements. To achieve this ultimate goal, the research activities conducted in FY 1995 are focussed on two major objectives: a) the characterization of the properties of foams and $b$ ) the detailed modeling of the foam performance.

The first issue to be addressed is the generation of a repeatable, controlled foam from a waterfoaming-agent-concentrate solution and air. A laboratory-scale apparatus has been designed and constructed which enables the generation of foam with a given expansion ratio (i.e., the ratio of the foam volume and of the liquid solution volume). Preliminary observations suggest that the operating pressure affect the air bubble distribution within the foam while having little impact on the overall expansion ratio (the higher the pressure the more uniform the bubble size). As to the ultimate effect of the bubble size distribution, insufficient evidence is available at this time to attempt any conclusion.

As the foam is generated, it immediately initiates to decay. While the foam product, used in these preliminary studies, could maintain reasonable structural properties for many hours after its generation, this behavior dramatically changes as the environmental conditions are altered (i.e., high temperature or presence of fire). Measurement techniques to characterize the foam density, thermal expansion coefficient, specific heat, thermal conductivity, radiation absorption coefficient and viscosity of the liquid solution were devised. Most of these measurements were conducted while significant changes occurred in the foam material especially at higher ambient temperature conditions. Analysis of the data collected is still ongoing. Some additional foam products are also being identified and similar measurements will be performed on several foams.

The detailed modeling of the foam behavior when exposed to a significant radiant flux from a fire has been completed under a set of simplifying assumptions. A dimensional analysis of the terms controlling the foam destruction process shows that, at high fluxes $\left(20 \mathrm{~kW} / \mathrm{m}^{2}\right.$ and above), a good portion of the destruction process can be approximated as quasi-steady state. For steady-state conditions, the most significant contribution to the foam vaporization process is given by the radiant flux which is absorbed at significant depth within the foam layer. The heat input causes the insitu vaporization of the liquid in the foam while little convective and conductive processes are present. This behavior of the foam is largely unexpected and provides most interesting perspectives for the future investigations.

The experimental apparatus design and constructed for the characterization of the foam behavior under fire radiant heat input will be used to study a variety of foam products. Extension of the foam performance measurement and evaluation to the weathering process before the fire exposures can also be accommodated in the existing apparatus and will be pursued along with the appropriate modeling developments.

\section{Recent Results}

Boyd, C.F. \& diMarzo, M., "Fire Protection

Foam in a Radiative Environment," accepted for presentation to the National Heat Transfer Conference, Houston, TX, August 1996. 


\section{A Survey of Occupant Load \\ Factors and Fuel Load in Contemporary Office Buildings}

\author{
Principal Investigator \\ James A. Milke \\ Department of Fire Protection Engineering \\ University of Maryland at College Park
}

\section{Sponsor}

National Institute of Standards and Technology

\section{Objective}

To provide a method of determining the occupant load in a variety of contemporary office buildings, i.e., open plan vs.

compartmented, public vs. private sector.

\section{Problem}

Questions have been raised concerning the accuracy of currently accepted values for occupant loads in office buildings. The concerns have resulted from preliminary studies conducted in open-plan and compartmented contemporary office buildings.

\section{Approach}

A literature survey was conducted to review the occupant load survey procedure and data collection forms used previously, e.g., by Bourdeau at the University of Maryland. This review resulted in the development of a preliminary data collection procedure and form for trial use. This approach was used to conduct an occupant load survey in public- and private-sector office spaces in the Washington metropolitan area.

In an occupant load analysis, two parameters are needed: the number of building occupants at any given time and the area of the floor space. Essentially, the approach consists of counting building occupants present along with indicating workstations which appear to be occupied normally. However, accounting for individuals absent from their workstation is a source of inaccuracy along with workers and guests moving throughout the building.

Many additional surveys are being conducted to expand the data base. Supplementing the building surveys will be analyses of space plans which show the number of workstations provided for each office. By comparing the results from the surveys with the space plans, a correlation will be derived to permit space plans to be used to estimate the occupant load.

\section{Recent Results}

Milke, J.A., Evaluation of Survey Procedures for Determining Occupant Load Factors in Contemporary Office Buildings, NIST-GCR-96697, National Institute of Standards and Technology, 1996.

Milke, J.A., A Survey of Fuel Loads in Contemporary Office Buildings, NIST-GCR-96698, National Institute of Standards and Technology, 1996. 



\section{FIRE SCIENCE DIVISION}

\section{FIRE SENSING AND EXTINGUISHMENT}





\section{Advanced Fire Detection}

Principal Investigator

William L. Grosshandler

Fire Science Division

301-975-2310

\section{Sponsor}

National Institute of Standards and Technology

\section{Objective}

To develop a test bed for evaluating the effectiveness of multi-point fire sensors and to demonstrate its usefulness for emulating standard smoldering fires.

\section{Problem}

Current test protocols and certification processes have evolved from smoke sensing principles established decades ago. New sensors that respond to different aspects of a fire are being developed for this and other applications that could open new vistas for the fire detection industry and provide enhanced protection for their customers. However, existing test methods may not accommodate these new designs nor are they necessarily equipped to evaluate those features which might make the designs attractive to markets.

\section{Approach}

During FY 1996, BFRL will develop a new method for simulating standard fires and evaluating fire detectors. The simulation method will have greater flexibility than existing methods, will perform the evaluation with more precision, and will accommodate multi-criteria decision-making algorithms. The new fireemulator/detector-evaluator (FE/DE) will consist of a heated wind tunnel with a test section $0.9 \mathrm{~m}$ wide by $0.3 \mathrm{~m}$ high and about 4 $\mathrm{m}$ long. The temperature and velocity will be controlled and the major gaseous products and particulate matter will be injected in a programmable manner to produce the desired levels at the detector location. Additional measurements will be taken in the two standard smoldering fires described in European Standard EN 54 part 9 to provide the proper input conditions for the FE/DE program.

\section{Recent Results}

Grosshandler, W. (ed.), Proceedings of the 1995

Workshop on Fire Detector Research, NISTIR 5700 , National Institute of Standards and Technology, June 1995.

Grosshandler, W., A Review of Measurements and Candidate Signatures for Early Fire Detection, NISTIR 5555, National Institute of Standards and Technology, January 1995.

Grosshandler, W., "Towards the Development of a Universal Fire Emulator/Detector Evaluator," 10. International Konferenz Über Automatische Brandentdeckung, Proceedings of $A U B E$ '95, H. Luck (ed.), Universität Duisburg, 1995.

Richards, R, Munk, B., Plumb, O., and Grosshandler, W., "Fire Detection and Location through Inverse Problem Solution," 10. Inter. Konferenz Über Automatische Branden., Proc. $A U B E$ '95, H. Luck (ed.), Universität Duisburg, 1995. 


\section{Fire Suppression Chemistry}

Principal Investigator

Gregory T. Linteris

Fire Science Division

301-975-2283

\section{Sponsor}

National Institute of Standards and Technology

\section{Objective}

To provide a fundamental understanding of the detailed chemical steps, by which the most

efficient chemical flame inhibitors act to extinguish flames, for the fire protection community to develop the next generation of suppression agents and technologies.

\section{Problem}

Fire suppressants and their application methods should be effective, fast-acting, economical, have low toxicity, permit a habitable environment during suppression, and cause no collateral damage to the protected space. Existing suppressants, however, are non-ideal: water from sprinklers can cause excessive damage, powdered suppressants leave a residue, and the currently favored suppressants, the halocarbons, produce corrosive gases after passing through a flame. There exists a continuing need for better suppressants and suppression methods, but limited scientific understanding of how existing chemical inhibitors act to extinguish flames hinders development of new agents. Some chemical inhibitors are much more effective, up to several orders of magnitude, than the best halogenated agent, $\mathrm{CF}_{3} \mathrm{Br}$. Although both gas-phase and heterogeneous chemical mechanisms have been proposed, the relative importance of each has not been discussed, and a clear understanding of the inhibition mechanism does not exist.

\section{Approach}

During FY 1996, BFRL will develop and understanding about inhibition chemistry of one super-effective chemical suppressant, iron pentacarbonyl, so knowledge of its mode of action can lead to development of other agents with more desirable properties. Experiments will determine the global properties and the detailed flame structure of the inhibited flames. The experimental results will be interpreted through numerical calculations of the flame structure. An existing counterflow diffusion flame code will be modified for inclusion of particle growth, and reaction.

Besides the work on iron pentacarbonyl, research on halogenated agents is continuing. Fluorinated hydrocarbons are being promoted as replacements for $\mathrm{CF}_{3} \mathrm{Br}$. A knowledge of the chemical behavior of fluorinated hydrocarbons in flames will promote their safe and efficient use in applications where deemed appropriate.

The experimental and modeling results from both investigations are expected to lead to a unified method of describing, and an improved fundamental understanding of the mechanisms of flame inhibition, extinction, and stabilization relevant to the advanced suppression of fires.

\section{Recent Results}

Smyth, Kermit, C., and Everest, David, A., The Effect of $\mathrm{CF}_{3} \mathrm{I}$ Compared to $\mathrm{CF}_{3} \mathrm{Br}$ on $\mathrm{OH}^{-}$and Soot Concentrations in Co-Flowing Propane/Air Diffusion Flames, for presentation at the 26th Symposium on Combustion, July 1996.

Reinelt, D., and Linteris, G.T., Experimental Study of the Flame Inhibition Effect of Iron Pentacarbonyl, Halon Optics Technical Working Conference, Albuquerque, NM, May 7-9, 1996.

Linteris, G. T., and Reinelt, D., "Inhibition of Flames by Condensed-Phase Agents," in Interflam' 96, Seventh International Fire Science and Engineering Conference, Cambridge, England, Interscience, London, pp. 477-486, March 26$28,1996$.

Linteris, G. T., and Truett, L. F., "Inhibition of Premixed Methane-Air Flames by Fluoromethanes," Combustion and Flame, 105: 15-27, 1996.

Linteris, G. T., "Numerically Predicted Flame Structure and Burning Rates of Premixed COAr-02-H2 Flames Inhibited by CF3H," accepted for publication in Combustion and Flame, January 1996. 
Reinelt, D., and Linteris, G. T., "Experimental Study of the Inhibition of Premixed and Diffuson Flames by Iron Pentacarbonyl," to appear in the Twenty-Sixth International Symposium on Combustion, The Combustion Institute, Pittsburgh, PA, January 1996.

Fallon, G. S., Chelliah, H. K, and Linteris, G. T., "Chemical Effects of CF3H in Extinguishing Counterflow CO/Air/H2 Diffusion Flames," to appear in the Twenty-Sixth International Symposium on Combustion, The Combustion Institute, Pittsburgh, PA, January 1996.

Linteris, G. T., and Truett, L., "Inhibition of Premixed Methane-Air Flames by Halon Alternatives," Proceedings of International Conference on Fire Research and Engineering, Orlando, FL, pp. 153-158, September 10-15, 1995.

Linteris, G. T., and Gmurczyk, G., "Inhibition of Premixed Methane-Air Flames by Iron Pentacarbonyl," 15th International Colloquium on the Dynamics of Explosions and Reactive Systems, pp. 298-301, Boulder, CO, July 30-August 4, 1995.

Babushok, V., Burgess, D. R, Linteris, G. T., Tsang, W., and Miziolek, A., "Modeling of Hydrogen Fluoride Formation from Flame Suppressants During Combustion," Halon Options Technical Working Conference, pp. 238250, Albuquerque, NM, May 9-1 1, 1995.

Linteris, G. T., and Gmurczyk, G., "Parametric Study of Hydrogen Fluoride Formation in Suppressed Fires," Halon Options Technical Working Conference, pp. 227-238, Albuquerque, NM, May 9-11, 1995.

Linteris, G. T., "Effect of Inhibitor Concentration on the Inhibition Mechanism of Fluoromethanes in Premixed Methane-Air Flames," Halon Replacements: Technology and Science, American Chemical Society Symposium Series, pp. 260-274, Washington, DC, 1995.

Linteris, G. T., "Acid Gas Production in Inhibited Propane-Air Diffusion Flames," Halon Replacements: Technology and Science, American Chemical Society Symposium Series, pp.
225-242, Washington, DC, 1995.

Linteris, G. T., and Gmurczyk, G., "Prediction of HF Formation During Suppression," in "Fire Suppression System Performance of Alternative Agents in Aircraft Engine and Dry Bay Laboratory Simulations," Editor Gann, R. G., NIST SP 890, pp. 201-318, National Institute of Standards and Technology, 1995. 


\section{Low Environmental Impact Fire Extinguishment}

\author{
Principal Investigator \\ William Grosshandler \\ Fire Science Division \\ 301-975-2310
}

\section{Sponsor}

National Institute of Standards and Technology

\section{Objective}

To facilitate the commercialization of promising low environmental impact fire suppression technologies by determining the impact of additives on enhancing vaporization during interaction of water droplets with a heated surface and developing a fixture for evaluating fire suppression efficiency of inert gas generators.

\section{Problem}

Current halon alternatives have ozone depletion potential and/or are significant greenhouse gases. Other approaches to fire protection have features that make them less desirable from an operational standpoint than the halons they are to replace. Certain additives to water have recently been shown to increase fire suppression efficiency. Inert gas generators have also been demonstrated to extinguish engine nacelle fires. Further development of these new technologies is hampered by a lack of understanding of the physical processes controlling their dispersion and by the absence of metrics for establishing their effectiveness.

\section{Approach}

During FY 1996, BFRL will perform experiments on single droplet impingement on a heated surface. The effects of additive concentration, droplet size, impact velocity, impact angle, and surface temperature on the vaporization process will be examined. Stroboscopic photography will be used to characterize droplet impact dynamics. Through consultations with academia, inert gas generators manufacturers, and potential end-users, a standard fixture for evaluating current and next generation of inert gas generators will be designed.

\section{Recent Results}

Grosshandler, W., Gmurczyk, G., and Presser, C., "Effectiveness of Halon Alternatives in Suppressing Dynamic Combustion Processes," in ACS Symposium Series, Halon Replacements:

Technology and Science, A. Miziolek and W. Tsang, Eds, 1995.

Grosshandler, W., "In Search of Alternative Fire Suppressants," accepted for C.L. Tien Symposium on Thermal Science, and "Engineering in Honor of Chancellor ChangLing Tian," Buckius, R O. Editor, p. 275-282, University of Illinois at Urbana-Champaign, November 14, 1995.

Grosshandler, W., "The Search for Environmentally Safe Fire Suppressants," seminar, Princeton University, February 1995.

Yang, J. C., and Grosshandler, W. C., Solid Propellant gas Generators: Proceedings of the 1995 Workshop, NISTIR 5766, National Institute of Standards and Technology, November 1995.

Grosshandler, W., and Gann, R., "Low Environmental Impact Fire Suppression Concepts," invited paper, Eastern States Section/Combustion Institute, December 1994. 


\section{Study of Technology for Detecting Pre-Ignition Conditions of Cooking- Related Fires Associated with Electric and Gas Ranges and Cooktops, Phase II}

\section{Principal Investigator}

Erik L. Johnsson

Fire Science Division

301-975-3083

\section{Sponsor}

The Consumer Product Safety Commission

Directorate for Engineering Sciences

\section{Objective}

To evaluate the methods, materials, and devices identified in FY 1995 (Phase I) and that can be used to detect the pre-ignition conditions identified in Phase I of cooking-related fires and alert, or intervene, to reduce the risk of cookingrelated fires associated with electric and gas ranges and cooktops.

\section{Problem}

In 1992, there was an estimated 98200 residential fires attributed to range/oven appliances. These fires resulted in an estimated 4670 injuries, 220 deaths, and property loss of $\$ 279$ million. Approximately two-thirds of the range/oven related fires involved food.

Currently, there is only a basic understanding of the pre-ignition conditions of food fires that might be used as indications of an incipient fire and of the performance of devices that might be used to detect and warn of such conditions.

\section{Approach}

During FY 1996, BFRL will addresses this work as two tasks:

1. First, information on the previously identified devices will be obtained, and the most promising technologies will be obtained for testing.

2. Second, the feasibility of differentiating between normal cooking and approaching fire conditions and the performance of the devices in recognizing the differences will be evaluated through a series of instrumented cooking experiments.

\section{Recent Results}

Johnsson, E. L., Study of Technology for Detecting

Pre-Ignition Conditions of Cooking-Related Fires

Associated with Electric and Gass Ranges and

Cooktops: Phase I Report, NISTIR 5729, National

Institute of Standards and Technology,

Consumer Product Safety Commission,

Washington, DC, p. 115, October 1995. 


\section{Fire Detection Strategies for High Bays}

\author{
Principal Investigator \\ William L. Grosshandler \\ Fire Science Division \\ 301-975-2310
}

\section{Sponsor \\ National Aeronautics and Space Administration NASA Headquarters}

\section{Objective}

To provide NASA with guidance on selection and placement of fire detectors for protecting high bay spaces with ceilings between $9 \mathrm{~m}$ and $18 \mathrm{~m}$.

\section{Problem}

Present guidelines for fire detector placement extend only to rooms with ceiling heights less than about $9 \mathrm{~m}$. NASA has a special need to address fire protection issues for high bay spaces because many facilities exist with ceiling heights greater than $9 \mathrm{~m}$, and in which a variety of functions are performed that are critical to the NASA strategic plan.

\section{Approach}

During FY 1996, BFRL will determine the response of smoke and heat detectors to jet fuel pool fires with heat release rates up to 12.5 MW using data from its full-scale tests conducted in a $15 \mathrm{~m}$ high aircraft hangar during 1995 under Department of the Navy sponsorship. The data will be put into a form that will allow comparisons of fire model predictions to the experimental results. The veracity of standard zone models such as BFRL's Consolidated Fire and Smoke Transport Model (CFAST), DETACT-QS and LAVENT will be challenged by comparing their predictions with the experiments in the $15 \mathrm{~m}$ high hangar. Algorithms to be tested include the predicted temperature structure of the ceiling jet and the temperature versus height and fire size of the plume models. Plume correlations not included in the zone model algorithms also will be compared with the experimental data. Suggested modifications of zone model algorithms will be documented based on comparisons of predictions with experiments. Modeling of the experimental data will also be done using HARWELL FLOW3D (a commercial package computer model that simulates fluid dynamic and heat transfer using a finite difference approach) and large-eddy-type simulations. Of primary interest is the comparison of plume temperature and ceiling jet temperature profiles using different turbulence approximations. In addition, ceiling jet velocity measurements will be compared with predictions of the CFD models.

\section{Recent Results}

Davis, W. D., Notarianni, K A., and Tapper, P. Z., "Modeling of Smoke Movement and Detector Performance in High Bay Spaces," Proceedings of International Conference on Fire Research and Engineering, Lund, D. Editor, Society of Fire Protection Engineers, pp. 307311 , September 1995. 


\section{Analysis of High Bay Hangar Facilities for Detector Sensitivity and Placement}

\section{Principal Investigator}

Darren L. Lowe

Fire Science Division

301-975-6667

\author{
Sponsor \\ Department of the Navy \\ Naval Facilities Engineering Command
}

\section{Objective}

To develop a comprehensive report of the full scale test results conducted during FY 1995.

The report will analyze experimental data on flame detection, smoke detection, and smoke movement in high bay aircraft hangars using aviation fuels so that decisions regarding appropriate fire protection for these spaces can be made.

\section{Problem}

Fire protection criteria for aircraft hangars employ "best engineering judgement" using NFPA codes and standards that are based on maximum ceiling heights of $9.1 \mathrm{~m}$. For years, $\mathrm{DOD}$ and the private sector have extrapolated data from research conducted in buildings with conventional ceiling heights. There are no actual data to support the current design practices for fire detection and suppression systems that may be $30.4 \mathrm{~m}$ or more above the fuel level. The effects of stratification, wind, and the movement of smoke and heat in high bay areas such as aircraft hangars are not presently known. Consequently, the reaction time and effectiveness of fire detection and suppression systems in aircraft hangars pose many questions.

\section{Approach}

During FY 1996, BFRL will write a report on research conducted in two high bay hangar facilities. The report will include descriptions of all detection systems used, building and test site specifications, test protocol, conditions, and analysis of all data gathered. The report will consist of two volumes, the first to be used as a guide or reference manual for Navy and DOD fire protection engineers, and the second volume will provide numerical results for validation of CFD models.

\section{Recent Results}

Conducted and analyzed full-scale tests to decide criteria for detector placement and the overall effectiveness of various type of detection systems.

Notarianni, K A., Gott, J. E., Davis, W., Lowe, D., and Laramee, S., "Analysis of High Bay Hanger Facilities for Detector Sensibility and Movement," C. Franks, and S. Grayson, Editors, Interflam '96, Interscience Communication Limited, London, pp. 487-496, 1996. 


\section{RECENT GRANTS - FIRE SENSING AND EXTINGUISHMENT}

\section{Detection of Fires Through Reflected Radiation}

\author{
Principal Investigator \\ Yudaya R Sivathanu \\ School of Mechanical Engineering \\ Purdue University \\ 317-494-9364
}

\section{Sponsor}

National Institute of Standards and Technology

\section{Objective}

To study a new type of fire detector that is based on a statistical analysis of the apparent source temperatures of natural fires and the power spectral density of the radiation emanating from them.

\section{Problem}

Current fire detectors include smoke, temperature, and radiation emission sensors. The smoke and temperature sensors detect fires based on the temperature or smoke density in its immediate vicinity. Therefore, a significant time delay is experienced between the initiation and the notification of the fire since the combustion products have to diffuse or convect to vicinity of the detector. In addition, in the presence of impermeable barriers, the fire is not detected even in its advanced stages.

A new type of fire detector being studied in the project uses two channels of information simultaneously. The radiation intensity emanating from a fire is measured at two closely space wavelengths in the near-infrared region of the spectrum. The detection of a fire is based on a statistical analysis of the resulting time series of apparent source temperatures.

During the design and construction of commercial buildings, front end evaluation of fire safety provisions are becoming increasing important. To assist architects in designing residential and commercial buildings with improved fire safety features, it is often necessary to determine apriori the optimum number of fire detectors and their locations within the structure. The second part of the present project involves developing a numerical model than can predict accurately the anticipated behavior of the near-infrared fire detector within an enclosure. Toward this end, a numerical model than can calculate the radiation intensity incident on the fire detector from direct and also reflected radiation is being developed. For realistic predictions of the response of the near-infrared fire detector, the optical properties of common building materiais and the geometrical shapes of typical residential or commercial structures must be used in the simulations.

\section{Approach}

The first task undertaken is characterizing the radiation emanating from the standardized test fires. The spectral radiation intensities emanating from the test fires at $900 \mathrm{~nm}$ and $1000 \mathrm{~nm}$ were measured using two photomultiplier tubes that had a $150^{\circ}$ view angle. The time series of spectral radiation intensities at these two wavelengths were converted to instantaneous apparent source temperatures assuming that the emissivity of the source varies inversely with wavelength. Spectral radiation intensity measurements have been completed for two of the six test fires. The two standardized test fires characterized so far in this project are a haptene pool flame and a foam smoldering fire. For these two fires, the apparent source temperatures obtained from direct and reflected radiation are between 1200 $\mathrm{K}$ and $2400 \mathrm{~K}$. The power spectral density (PSD) of the apparent source temperatures of these fires show that most of the energy of the fluctuations is concentrated at low frequencies (between $0 \mathrm{~Hz}$ and $40 \mathrm{~Hz}$ ). This is typical of most natural fires. Therefore, an algorithm which gives a positive response if 40 percent of the apparent source temperatures are between $1200 \mathrm{~K}$ and $2400 \mathrm{~K}$ and 40 percent of the energy of the fluctuations is between $0 \mathrm{~Hz}$ and $40 \mathrm{~Hz}$ have been chosen as a trial fire detection algorithm. This algorithm will be tested using the standardized test fires both for sensitivity to starting fires and for incidences of false alarms. 
The numerical model to determine the response of the fire detector involves the computation of the radiation intensity incident on it from fires within an enclosure. A statistical approach is used to calculate a large number of photon trajectories from the fire to the detector, including multiple interactions with the walls of the enclosure. The probability associated with the photon trajectories are calculated using a discrete probability function method which substantially reduces the errors introduced by conventional Monte-Carlo simulations. Initial calculations using the discrete probability function method for an axisymmetric geometry has been completed.

\section{Recent Results}

Sivathanu, Y. R, and Tseng, L. K,

"Characterization of Radiation Properties of Fires Using Multi-Wavelength Measurements," Fall Technical Meeting of the Central States Section of the Combustion Institute, 1996.

Sivathanu, Y. R, and Gore, J. P., "Radiative Heat Transfer Inside a Cylindrical Enclosure," Symposium on Fire and Combustion Systems, 1996 International Mechanical Engineering Congress and Exposition.

\section{A Theoretical and Experimental Investigation on Physical, Thermal and Chemical Effects of Condensed Phase Fire Suppressants}

\author{
Principal Investigator \\ Harsha K. Chelliah \\ Department of Mechanical, Aerospace \\ and Nuclear Engineering \\ University of Virginia \\ 804-924-6037
}

\section{Sponsor}

National Institute of Standards and Technology

\section{Objectives}

To quantify the physical, thermal and chemical effects of condensed-phase fire suppressants in extinguishing counterflow laminar flames by experimental and computational techniques.

\section{Problem}

The production of chemical fire suppressants containing Br-atoms (e.g., CF3Br halon 1301) has been banned because of its adverse effects on the upper-atmospheric ozone layer. On a mass basis, sodium bicarbonate $(\mathrm{NaHCO} 3)$ is known to be more effective than halon 1301, certainly, far more effective than most alternate gas-phase fire suppressants. Yet, the underlying physical, thermal and chemical effects of such condensed-phase suppressants are understood only at a qualitative level.

Recent experimental results with varying sizes of $\mathrm{NaHCO} 3$ particles have shown counter intuitive trends on suppression effectiveness. To understand these results and provide information valuable in the search for alternative fire suppressants, a comprehensive experimental and theoretical approach is adopted in the present work.

\section{Approach}

Nonpremixed flames of hydrocarbon fuels and air, established in a steady, laminar, counterflow field, provide an excellent configuration for theoretical and experimental studies directed at better understanding the fundamental characteristics of fire suppressing agents. This 
configuration is especially useful for studies involving condensed-phase agents, such as sodium bicarbonate powder $(\mathrm{NaHCO} 3)$ and water droplets, because of the complex interactions occurring between the two phases.

For example, introduction of condensed phase fire suppressants with air or fuelstreams can exert physical effects through drag forces and modification of transport properties, thermal effects through heats of decomposition, sublimation, vaporization, modification of heat capacity, thermal radiation, etc., and chemical effects through the inhibition reaction pathways (both homogeneous and heterogeneous). Based on detailed theoretical models, extraction of the dominant contributions from the above list requires careful validation of the model with experimental data, for a wide range of conditions. For planar flames established within the mixing layer, the axial velocity gradient, or the flow strain rate, at flame extinction provides a convenient parameter to quantify the suppression effectiveness.

Numerical calculations are being performed to determine the chemical, thermal and physical contributions of $\mathrm{NaHCO} 3$ and fine water particles on the extinction strain rate. An Eulerian-Eulerian formulation is adopted to describe the two-phase conservation equations. As part of this study, the chemical kinetic, thermodynamic and transport data of the intermediate gaseous products of $\mathrm{NaHCO} 3$ are being compiled and the chemical effectiveness of some of these gaseous intermediates in suppressing counterflow nonpremixed flames has already been investigated. In particular, the strain rate at extinction of counterflow $\mathrm{CH} 4 /$ air nonpremixed flame was shown to decrease by 12 percent due to the addition of 0.1 percent $\mathrm{NaOH}$ by mass to the air stream. With the two-phase flame code being developed, not only these chemical effects, but other physical and thermal effects will be investigated and detailed comparisons with experimentally measured extinction strain rates will be made. In the supporting experiments, an enclosed, counterflow burner has been designed and is being built with provision for introducing condensed-phase suppressing agents with the air-stream. The enclosed feature of the burner is mainly for safety reasons, but could be used to investigate pressure effects as well. An interchangeable fuel feed system is being developed to test the suppression effectiveness of condensed phase agents against gaseous and liquid fuels. Initial 2D simulations have shown that non-uniform flow at the jet exit can affect the flame shape. Because of the seeding of the air stream with the condensed phase agents, use of screens is not an option to make the flow uniform. Therefore, con verging nozzles will be used to obtain uniform flow at the jet exit. Additional nitrogen shroud flow is provided for dilution and for avoiding secondary flames. The focusing and collecting optics of the LDA system are mounted on Klinger stages (with rated accuracy of $2 \mathrm{~m}$ ) and is traversed up and down to map the velocity field. During the first year, the goal is to characterize the effectiveness of various sizes of $\mathrm{NaHCO} 3$ particles (ranging from 1 to $50+{ }^{~} \mathrm{~m}$ ) in suppressing nonpremixed gaseous flames, based on the local extinction strain rate measurements using the LDA system. In subsequent years, advanced laser diagnostic facilities will be used for point measurements and planar imaging of the temperature and species profiles to further validate the numerical model.

\section{Recent Results}

New project 


\section{Investigation of Droplet Penetrations Through Complex Openings of Compartments}

Principal Investigator

Prof. S. C. Yao

Department of Mechanical Engineering

Carnegie Mellon University

Sponsor

National Institute of Standards and Technology

\section{Objective}

To understand the phenomena and mechanisms of the mist penetration through openings of compartments.

\section{Problem}

Fire frequently starts from equipment compartments. Yet when mist sprays are activated, droplets could be hard to penetrate the openings. The process is complex. For large droplets, their motions are governed by inertia, therefore, interception and liquid film reentrainment could occur. For small droplets their motions follow the air stream closely and may not be able to penetrate the compartment substantially. Furthermore, the structures of the compartment openings are usually complex.

\section{Approach}

Experimental and analytical studies will be conducted systematically to 1 . investigate the ability of droplets penetrating the openings directly under the effects of aerodynamic drag, inertia and gravity at various conditions; 2 . examine the droplet impaction dynamics to structures that the indirect penetration following the impaction can be estimated; and 3 . assess the overall liquid penetration efficiency.

The studies include:

1. creating a mono-size mist generator using Impulsed Spray Generator $[1,2]$ invented at CMU,

2. detailed observation on the impaction phenomena of mono-size droplets [3,4] on cylindrical wires, screens and slots at wide range of operations. The processed images will provide understandings and also non-dimensional correlations, and 3 . analytical modeling of the process in 2-D configurations.

\section{Recent Results}

New project 



\section{FIRE SCIENCE DIVISION}

\section{MATERIALS FIRE RESEARCH}





\section{Flammability of Structural Composites}

\author{
Principal Investigator \\ Tom Ohlemiller \\ Fire Science Division \\ 301-975-6481
}

\section{Sponsor}

National Institute of Standards and Technology

\section{Objective}

To develop quantitative procedures for assessing the impact of fire on structural composites with the ultimate goal of materials guidelines that assure fire safety.

\section{Problem}

Composites are highly attractive materials for a variety of structural uses but their organic resin content renders them potentially flammable and subject to loss of strength in a fire. These two areas of vulnerability may be coupled since mechanical loading can yield delaminations which alter thermal properties. Thus, the general problem of composite response to fire is extremely complex and we are forced to approach it in stages. BFRL researchers first focused on the problem of fire growth over the surface of a composite and to understand the conditions which can assure that this growth is minimal. Ideally, this threat should be assessable from bench-scale tests on the composite, coupled with appropriate models of fire growth using inputs from these small-scale tests. BFRL researchers pursued this approach in previous work for the Department of Navy and have had reasonable success in assessing the potential for upward flamespread on flat vertical wall surfaces, using models developed for other materials. However, that work showed that a typical candidate composite for infrastructure usage, a flame-retarded vinyl ester/glass material, is susceptible to fire growth. Thus, BFRL has explored further technologies for suppressing surface fire growth. A related problem is a consensus on appropriate fire testing technology of composites used for infrastructure applications; we will be pursuing industry input on this issue.

\section{Approach}

During FY 1996, BFRL will address the issue of whether fire can spread on a composite. Once suppression of spread is assured, the issue of fire endurance to a localized fire exposure remains; this work will be pursued later. BFRL will continue to address the control of fire spread by means of surface coatings (since this appears more promising than composite resin formulation changes), identifying promising coatings in bench-scale tests then assessing their efficacy by full-scale checks. BFRL will examine charring, charring/intumescent and ceramic coatings in its Cone Calorimeter and LIFT apparatuses for their ability to delay ignition, depress the rate of heat release and limit lateral fire spread. A successful coating must do all these things and it must remain firmly attached to the face of the composite despite severe heat stresses. In the near future, the focus of this research will study further aspects of coating technology and the assurance of appropriate noload bench-scale and full-scale tests for fire spread suppression. Mechanical loading may impact the efficacy of a coating (strain could dislodge the coating); this work will be performed in a later year.

\section{Recent Results}

Ohlemiller, T., Cleary, T., and Shields, J., "Effect of Ignition Conditions on Upward Flame Spread on a Composite Material in a Corner Configuration," Proceedings of the 41st International SAMPE Symposium, Vol. 41, Book 1, SAMPE, p. 734, 1996. 


\section{Radiative Ignition and Subsequent Flame Spread Over Cellulosic Materials}

\author{
Principal Investigators \\ Takashi Kashiwagi \\ Fire Science Division \\ 301-975-6699
}

Howard Baum

Fire Safety Engineering Division

301-975-6668

Kevin McGrattan

Fire Safety Engineering Division

301-975-2712

\section{Sponsor}

National Aeronautics and Space Administration Lewis Research Center

Microgravity Science Program

\section{Objective}

To develop new models that describe the characteristics of heat transfer and flame spread along a narrow diameter polymer rod in microgravity.

\section{Problem}

This project is one of NASA Microgravity Science projects to enhance understanding of combustion science by taking unique advantage of a microgravity environment. Knowledge is lacking about the ignition and subsequent flame spread over a thermally thin cellulosic material in a microgravity environment.

\section{Approach}

During FY 1996, BFRL will develop and debug a new numerical code based on the full NavierStokes equations as an extension from the current code based on the potential flow approximation. The model will be extended to include smoldering phenomena along a thin paper. The experimental design, fabrication, experimental parameters and procedures are being developed with researchers at NASA Lewis Research Center for BFRL's experiments in the planned USMP-3 Space Shuttle flight. Experiments in high oxygen atmospheres will be conducted using the Japanese 10 seconds drop tower in March 1996 as an extension to the previous study.

\section{Recent Results}

McGrattan, K B., Kashiwagi, T., Baum, H. R, and Olson, S. L., "Ignition and Wind Effects on the Transition to Flame Spread in a Microgravity Environment," Combustion and Flame, in press.

Nakabe, K, McGrattan, K B., Kashiwagi, T., Baum, H. R, Yamashita, H., and Kushida, G., "Ignition and Transition to Flame Spread Over a Thermally Thin Cellulosic Sheet in a Microgravity Environment," Combustion and Flame, 98: 361-374, 1994. 


\section{Combustion of a Polymer (PMMA) Sphere in Microgravity}

\section{Principal Investigator}

Jiann C. Yang

Fire Science Division

301-975-6662

\section{Sponsor}

National Aeronautics and Space Administration Lewis Research Center

\section{Objective}

To determine the burning rates of PMMA spheres in a low gravity environment under various ambient oxygen conditions

\section{Problem}

The need to use polymeric materials that may be flammable cannot be eliminated aboard the space shuttle or the future manned permanent space-station. Knowledge is needed to understand potential fire threats caused by the presence of polymeric materials. The use of spherical geometry has many advantages -- a spherical flame, which is one-dimensional, is amenable to simple mathematical analysis and facilitates a detailed examination of the many interesting phenomena associated with the combustion of a solid phase material without involving the complications of flame-spread, which is still an active research area.

\section{Approach}

During FY 1996, BFRL will perform fire experiments using polymeric materials in reduced gravity achieved aboard a DC-9 aircraft flying a parabolic trajectory. The sphere will be ignited using a pair of opposing micro-torches. A mechanism for deploying an unsupported sphere will be developed. Experiments using suspended spheres also will be performed. Suspended spheres will be obtained by supporting the spheres at the tip of a thermocouple. Results will be compared with those obtained under normal gravity condition. NASA will provide technical assistance to the design phase of the experiment and will conduct flight experiments.

\section{Recent Results}

Yang, J. C., and Hamins, A., "Combustion of a Polymer (PMMA) Sphere in Microgravity," Third International Microgravity Combustion Workshop, April 11-13, 1995. 


\section{Chemical Inhibitor Effects on Diffusion Flames in Microgravity}

\author{
Principal Investigators \\ Gregory T. Linteris \\ Fire Science Division \\ 301.975-2283 \\ Professor Simone Hochgreb \\ Massachusetts Institute of Technology
}

Sponsor

National Aeronautics and Space Administration Lewis Research Center

Space Systems Branch

\section{Objective}

To determine the effects of flame inhibitors on the physical characteristics (height, shape, color, and luminosity) and stability limits (ignition, extinction, lift off and blow-off) of gaseous diffusion flames in the presence of halogenated fire suppressants in microgravity; and to develop quantitative analytical models for the observed behavior, including chemical kinetic effects, to understand the mechanisms of inhibition of halogenated compounds in gaseous diffusion flames in microgravity.

\section{Problem}

Fire safety in space is one main reason to investigate combustion phenomena in microgravity. Since halogenated compounds will remain the fire suppressant of choice in many space missions, there is a need for experimental evidence on the effectiveness of these compounds for diffusion flames under zero buoyancy conditions.

\section{Approach}

During FX 1996, BFRL will perform experiments of normal and microgravity studies of laminar jet and co-flow diffusion flames inhibited by halogenated suppressant in the air or fuel stream. In microgravity, visual and temperature diagnostics will be used to detect flame shape, dynamics, and stability limits. Normal gravity experiments will use additional chromatographic and spectroscopic diagnostics besides visual and temperature experiments. Analytical and computational work will be performed using existing general 2-D codes with chemical kinetic models for comparison with the observed results.

\section{Recent Results}

VanDerWege, B. A., Bush, M. T., Hochgreb, S., and Linteris, G. T., "Effect of CF3H and CF3Br on Laminar Diffusion Flames in Normal and Microgravity," Third International Microgravity Combustion Conference, Cleveland, OH, April 1113, 1995.

VanDerWege, B. A., Bush, M. T., Hochgreb, S., and Linteris, G., "Effect of CF3H and CF3Br on Laminar Diffusion Flames in Normal and Microgravity," Eastern States Section Meeting/The Combustion Institute, Worcester, MA, October 1995. 


\section{Combustion of Silicone}

\author{
Principal Investigator \\ Takashi Kashiwagi \\ Fire Science Division \\ 301-975-6699
}

\section{Sponsor \\ Dow Corning Co.}

\section{Objective}

To understand the combustion mechanism of siloxane and specifically why the heat release rate (as measured in the cone calorimeter) of burning siloxane is nearly independent of external thermal radiant flux.

\section{Problem}

The heat release rate (HRR) of burning materials increases significantly with an increase in external thermal radiant flux applied to the burning surface. This is true for non-charring materials and for charring materials. However, the burning behavior of siloxanes differs markedly from carbon-based materials. Perhaps most significant, the HRR for siloxanes (chain length $>15 \mathrm{~S}_{\mathrm{i}}-\mathrm{O}$ units) does not increase significantly with an increase in external thermal radiant flux. Whereas carbon-based materials may form products of incomplete combustion such as soot and carbonaceous chars, siloxanes form very minimal char but produce substantial amounts of solid amorphous silica $\left(\mathrm{S}_{\mathrm{i}} \mathrm{O}_{2}\right)$ as a major product of combustion. Previous studies suggest that amorphous silica play a significant role in mediating the burning behavior of siloxanes.

\section{Approach}

During FY 1996, BFRL will measure emission spectra of silica/silane/PDMS flames. First, the emission spectra of high temperature silica particles will be measured at visible and IR wavelengths. Second, the emission spectra of a small silane flame to observe emission bands in molecules containing silicon, such as $\mathrm{S}_{\mathrm{i}} \mathrm{O}$.

Third, a flame burning D4 or other short length silicone fluid will be used to repeat the spectral measurements to determine if other emission bands exist, as compared with the bands observed in the silica and the silane measurements.

Next, BFRL will analyze evolved gaseous products and fluid residues during gasification of silicones to understand their gasification processes. Evolved gaseous products of several silicone fluids (e.g., D4, $50 \mathrm{cs}, 1000 \mathrm{cs}$ ) exposed to two different external radiant fluxes in nitrogen will be trapped at different gasification stages and the products will be analyzed to identify them.

\section{Recent Results}

Buch, R., Hamins, A., Konishi, K, and Kashiwagi, T., "Mass Vaporization Rates and Radiactive Emission of Pool Burning of Silicone Fluids," submitted to Combustion and Flame, 1996. 


\section{Lean Flammability Limit as a Fundamental Refrigerant Property, Phase II}

\author{
Principal Investigator \\ Carole Womeldorf \\ Fire Science Division \\ 301-975-4415
}

Sponsor

Air-Conditioning and Refrigeration Institute

\section{Objective}

To accurately evaluate the lean flammability limits of various refrigerant mixtures (R-32, R125, R-134a, and R-245ca) using an improved counterflow burner.

\section{Problem}

A critical element of the risk analysis of alternative refrigerants is the lean flammability limit, which ASTM E681-85 determines.

Variations in flammability limits are observed depending on the initial conditions chosen for conducting the test. There is a need to eliminate these variations and to establish invariant, fundamental lean flammability limits (LFL) which can be attributed to refrigerant mixtures similar to fundamental thermodynamic properties like the adiabatic flame temperature.

\section{Approach}

During FY 1996, BFRL will develop methods to permit the aerodynamically improved burner to run with a fully automated flow control system. Computer control of all flows will permit precise evaluation of extinction points for preset strain rates or equivalence ratios. The new burner will be calibrated using $\mathrm{CH}_{4}$ to ensure that the facility can reproduce the published values of the lean flammability limit. The lean flammability limit of HFC-32 and air will then be examined for different humidities and geometries. The fuel/air equivalence ratio will be varied about the stoichiometric point and the strain rate will be adjusted to identify the most robust mixture (normally slightly rich for hydrocarbon flames). The lean flammability limit of HFC-32 will be determined using the zero strain rate extrapolation method. The critical flammability ratio (CFR) will be found by slowly increasing the fraction of HFC- 125 in the HFC/air mixture while maintaining the overall equivalence ratio and strain rate fixed, or by fixing the proportions of all constituents and slowly increasing the strain rate by increasing the total flow. Numerical results from CHEMKIN will provide insight into the kinetic mechanisms of HFC-32 as a fuel and predictions of the reduction of flame speed caused by the addition of non-flammable refrigerants.

\section{Recent Results}

Womeldorf, C., and Grosshandler, W., Lean Flammability as a Fundamental Refrigerant Property, Phase II, DOE/CE/238 10-68, Department of Energy, April 30, 1996. 
Calibration Facility for Particle Size and Concentration

Principal Investigator

George W. Mulholland

Fire Science Division

301-975-6695

\section{Sponsor}

National Institute of Standards and Technology

\section{Objective}

To develop a facility for accurately measuring particle size and concentration and for depositing monodisperse particles of specified size, number, and chemistry on calibration artifacts.

\section{Problem}

The detection, quantification and characterization of particulate contamination on semiconductor surfaces is essential to advanced semiconductor manufacturing. The present practical limit for particle detection is $0.1 \mu \mathrm{m}$ diameter. The National Technology Roadmap for Semiconductors calls for the ability to detect and quantify particles with $0.06 \mu \mathrm{m}$ equivalent diameter by 2001 . There is a need for accurately sized monodisperse particles in the size range from $0.07 \mu \mathrm{m}$ to 0.2 $\mu \mathrm{m}$ for developing and calibrating improved wafer scanners.

\section{Approach}

During FY 1996, BFRL will collaborate with NIST's Electronic and Electrical Engineering Laboratory (EEEL) to calibrate a voltage divider to provide an accurate calibration tool for measuring the collector rod voltage of the differential mobility analyzer (DMA). This is essential for performing accurate measurements with the DMA. An experimental design will be developed for accurately sizing four sample sizes with nominal diameters of $72 \mathrm{~nm}, 89 \mathrm{~nm}, 125$ $\mathrm{nm}$, and $180 \mathrm{~nm}$ by EEEL and the University of Minnesota. Final selection of the samples to be measured will be made after completing electron microscopy analysis. Screening measurements were completed last year on 22 samples using the DMA. Upon completion of the measurements and analysis, a paper will be written and the particles will be available for purchase.

\section{Recent Results}

Four calibration sizes are available for the calibration of surface scanners and other particle sizing instrumentation. 


\section{Burning Rate of Real Materials}

\author{
Principal Investigators \\ Takashi Kashiwagi \\ Fire Science Division \\ 301-975-6699 \\ Ken Steckler \\ Fire Science Division \\ $301-975-6678$ \\ Kathy Butler \\ Fire Science Division \\ 301-975-6673 \\ Steve Ritchie \\ Fire Science Division \\ $301-975-5441$
}

\section{Sponsor}

National Institute of Standards and Technology

\section{Objective}

To determine global parameters that can represent gasification characteristics of single component materials, for use in calculating burning rates of the real materials when heat feedback rates is provided, and tabulate their values for many plastics and to apply results to composite materials (e.g., a fabric with a foam, mattresses, fiber reinforced composites).

\section{Problem}

Now we do not have models that can calculate the burning rate (heat release rate) of real materials such as buildings contents. Therefore, a fire source is generally prescribed or estimated from bench scale tests. To improve the prediction of fire hazards, accurate description of fire sources is urgently needed.

\section{Approach}

During FY 1996, BFRL will derive the global parameters from the comparison of measured gasification rates of selected real materials and theoretically calculated weight loss rates. The gasification rates will be measured in nitrogen atmosphere under several different radiant fluxes. One dimensional heat conduction model with a global degradation reaction will be nondimentionalized and key parameters (hopefully as low as two) will be defined. The weight loss rates will be calculated at the external fluxes as a function of the parameters.

\section{Recent Results}

Choi, M. Y., Mulholland, G. W., Hamins, A., and Kashiwagi, T., "Comparison of the Soot Volume Fraction Using Gravimetric and Optical Techniques," Combustion and Flame, 102: 161 169, 1995.

Choi, M. Y., Hamins, A., Rushmeier, H., and Kashiwagi, T., "Simultaneous Optical Measurement of Soot Volume Fraction, Temperature, and $\mathrm{CO}_{2}$ in Heptane Pool Fires," Twenty-Fifth Symposium (International) on Combustion, The Combustion Institute, 1471 1480, 1994.

Kashiwagi, T., "Role of the Condensed Phase in Polymer Combustion and Flammability," Twenty-Fifth Symposium (International) on Combustion, The Combustion Institute, 14231437, 1994. 


\section{High Heat Flux Measurement Standards}

\author{
Principal Investigator \\ William L. Grosshandler \\ Fire Science Division \\ 301-975-2310
}

\section{Sponsor}

National Institute of Standards and Technology

\section{Objective}

To develop a convective heat transfer facility for calibrating heat flux gauges, to develop a concept for a gas phase conduction facility to extend the calibration range to $100 \mathrm{~kW} / \mathrm{m}^{2}$, and to develop for industry, new techniques for applications to critical technologies.

\section{Problem}

Standard methods exist for calibrating thermal radiation detectors at flux levels extending to 10 $\mathrm{kW} / \mathrm{m}^{2}$ using controlled blackbody cavities, and up to $40 \mathrm{~kW} / \mathrm{m}^{2}$ using a monochromatic laser radiation source. However, heat flux transducers are often used under conditions where convection is dominant, where radiation emanates from a source with different spectral character, or where the flux levels exceed 40 $\mathrm{kW} / \mathrm{m}^{2}$.

\section{Approach}

During FY 1996, BFRL will complete construction of an experimental facility consisting of a wind tunnel with a heated wall in which a heat flux transducer can be mounted flush. Air at a cooler temperature will flow through the duct in a precisely controlled manner. The gas temperature and velocity will be variable, with heat fluxes at the surface designed to be preset between $0.5 \mathrm{~kW} / \mathrm{m}^{2}$ and $5.0 \mathrm{~kW} / \mathrm{m}^{2}$ within an uncertainty of less than \pm 5 percent. Flow visualization, hot-wire probing, thermocouples, and temperature sensitive liquid crystals will be used to measure the temperature fields in the air and on the surface beside the flux gauge. Numerical simulations by NIST coworkers in the Chemical Science and Technology Laboratory and the Electronic and Electrical Engineering Laboratory will be consulted to refine the design and minimize uncertainties. In a second facility, the guarded hot-plate concept will be extrapolated to produce heat fluxes up to $100 \mathrm{~kW} / \mathrm{m}^{2}$. A 1.0 $\mathrm{mm}$ thick layer of helium will separate two flat, polished copper plates maintained at a temperature difference of several hundred degrees. This type of construction should minimize the contribution of radiation and convection to the conductive heat transfer.

\section{Recent Results}

Moffat, R. J., and Darek, C., Final Report on the NIST/NSF Workshop on Heat Flux Gage Calibration, unpublished (available from W. Grossh andler, BFRL, NIST, Gaithersburg, MD 20899), January 23-24, 1995. 


\section{Evaluation of Fire Barrier Performance}

\author{
Principal Investigator \\ Kenneth D. Steckler \\ Fire Science Division \\ 301-975-6678
}

Sponsor

U.S. Nuclear Regulatory Commission

Office of Nuclear Reactor Regulation

\section{Objective}

To provide technical support to Nuclear Regulatory Commission (NRC) staff in review and evaluation of technical issues to fire-barrier systems in nuclear power plants.

\section{Problem}

The NRC requires technical assistance for evaluating the fire performance of certain fire barrier systems intended to satisfy the fire protection of safe shutdown capability as specified in certain NRC documents.

\section{Approach}

During FY 1996, BFRL will conduct a series of reduced-scale fire-endurance tests on fire-barrier systems in use in U.S. nuclear power plants (NPP). A series of mechanical tests will be performed on subliming fire-barrier materials to confirm the mechanical properties provided by the vendor of the subject materials. BFRL will integrate into a single document three of its 1994 NRC Letter Reports on the history of the development of the ASTM E-1 19 temperaturetime fire-severity curve, the history of past efforts to develop curves for other specific applications; and an assessment of the feasibility of developing and implementing nuclear-power-plant (NPP)-specific fire-severity curves.

\section{Recent Results}

Cooper, L., Y., and Steckler, KD., Methodology

for Developing and Implementing Alternative

Temperature-Time Curves for Testing the Fire

Resistance of Barriers for Nuclear Power Plant

Applications, NISTIR 5842, National Institute of Standards and Technology, May 1996.

\section{Furniture Flammability}

\author{
Principal Investigator \\ Tom Ohlemiller \\ Fire Science Division \\ 301-975-6481
}

\section{Sponsor}

National Institute of Standards and Technology

\section{Objective}

To develop the technical basis, ultimately expressed as guidelines for furniture manufacturers, to design upholstered furniture with a controlled rate of heat release.

\section{Problem}

Current furniture, particularly for residences, can produce large, rapidly growing fires that can lead to room flashover. Lacking are viable techniques to preclude this flashover phenomenon by limiting the peak rate of heat release. Since other materials, such as carpeting and drapes can rapidly contribute to the heat release from a chair fire, allowable peak heat release from upholstered furniture should not exceed $500 \mathrm{~kW}$. The recent European Community Combustion Behavior of Upholstered Furniture study (ECCBUF) showed that most residential-type furniture undergoes very non-ideal behavior before reaching its heat release rate peak (e.g., large geometry changes occur or a floor pool fire occurs). The CBUF study did not offer viable modeling alternatives. Recent efforts at correlations between Cone Calorimeter data and full-scale performance have had some success but there are indications that existing correlations are of limited validity. They have not been well enough guided by a physical understanding of the problem.

\section{Approach}

During FY 1996, BFRL will attempt to relate Cone Calorimeter and LIFT behavior to fullscale through appropriate models. This is made more attractive because of one outcome of the CBUF study - the development of a technique to predict Cone furniture composite behavior from the Cone behavior of the individual materials. The models and supporting 
experiments will be developed in stages, beginning with isolated, single flat cushions, either horizontal or vertical. The modeling effort will attempt to take advantage of associated BFRL research related to room surface fires. The goal, for each cushion surface orientation, is an adequate semi-quantitative or even correctly qualitative model which captures the important parameters for a fire growing on such a cushion from an arbitrary ignition condition. Once the modeling for isolated cushions, as verified by comparisons with experiments, succeeds in capturing all of the qualitative dependencies of the heat release rate history, an attempt will be made to develop a quantitative correlation between the input parameters, inferred from the bench-scale tests, and full-scale behavior. This is roughly the type approach BFRL used to correlate successfully a limited portion of its previous chair results (the most ideally behaved subset). Next, this approach will be applied to an interacting pair of cushions (seat and seatback). The next step is to elevate the pair of cushions on a framework to introduce the possibility of a floor fire (usually a consequence of dripping polyurethane melt) which interacts with the cushion fire. At each step, the modeling process is expected to help identify the main parameters; the model is not expected to develop quantitative predictions of the heat release behavior of a variety of chairs. The role of the correlations is to capture this in an empirically determined quantitative manner, useful to manufacturers.

\section{Recent Results}

Ohlemiller, T. J., "An Examination of the Correlation Between Cone Calorimeter Data and Full-Scale Furniture Mock-Up Fires," Proceedings of the International Conference on Fire Research and Engineering, Society of Fire Protection Engineers, p. 217, Boston, MA, 1995.

\section{Test Method Development for Cigarette Ignition Propensity}

\author{
Principal Investigator \\ Richard G. Gann \\ Fire Science Division \\ 301-975-6866
}

\section{Sponsor}

National Institute of Standards and Technology

\section{Objective}

To respond to issues about test methods for cigarette ignition propensity and toxic potency measurement.

\section{Problem}

BFRL completed a method for measuring the relative likelihood that a cigarette will ignite soft furnishings. Expected comments on the method will require analysis and response. BFRL also completed work on a test method for the toxic potency of smoke from burning products. The method is under discussion in ASTM and NFPA. Participation in these activities will require presentations of further analysis of the research.

\section{Approach}

During FY 1996, BFRL and NIST's Information Technology Laboratory will prepare responses, as needed, to issues about the methods. The responses are expected to require further analysis of the data.

\section{Recent Results}

BFRL's research served as the bases for three Standards:

1. Cigarette ignition propensity test method, introduced into ASTM E-5.15 by ATMI,

2. Smoke toxic potency test method (NFPA 269), has cleared the Fire Test Committee, and

3. Revised version of ASTM E-1678 was constructed to include both pre- and postflashover fire evaluations. 


\section{Polymeric Film Applied to Glass}

\author{
Principal Investigators \\ Jeff Gilman \\ Fire Science Division \\ 301-975-6573 \\ Emil Simiu \\ Structures Division \\ 301-975-6076
}

\section{Sponsor}

General Services Administration

Public Building Services

\section{Objective}

To search English language bibliographic databases for research reports, test data or other available information in the literature on: 1) blast effects on architectural glass; 2) performance of polymeric film on glass under blast conditions; 3 ) the application, durability and maintainability of polymeric films on glass, and prepare a report for the General Services Administration (GSA) summarizing the literature found in the search.

\section{Problem}

A report by the Department of Justice, Vulnerability of Federal Facilities, prompted by the terrorist attack in Oklahoma City, calls for the application of polymeric film to exterior glass in Federal buildings to reduce the possibility of damage due to flying glass from explosive blasts. However, before undertaking an extensive retrofit of all Federal facilities, GSA requested BFRL's technical assistance to study this topic and report the findings.

\section{Approach}

During FY 1996, BFRL will perform on-line

literature searches of four databases:

- RAPRA Abstracts (1972-1995/Aug., 1995

RAPRA Technology Ltd., Engineering Index);

- Ei Compendex Plus (TM) (1970-1995/Oct., 1995 Engineering Info. Inc.);

- National Technical Information Service (NTIS) Database, (1964-1995); and

- Architectural Database (1978-1995).
Relevant keywords such as; blast, explosion, impact, glass, polymer, film, coating, hurricane, and earthquake were searched. A report summarizing this literature was written. The report includes a bibliography.

\section{Recent Results}

Gilman, J. and Simiu, E., Polymer Film Applied to Glass: Effectiveness at Mitigating Damage from Flying Glass due to Explosions, NISTIR 5779 , National Institute of Standards and Technology, January 1996. 
RECENT GRANTS - MATERIALS FIRE RESEARCH

\section{Flame Resistance of Benzocyclobutene (BCB) Functionalized Copolymers}

\author{
Principal Investigator \\ David C. Martin, Ph.D. \\ Materials Science and Engineering \\ The University of Michigan
}

\section{Sponsor}

National Institute of Standards and Technology

\section{Objective}

To critically evaluate the influence of benzocyclobutene (BCB) functionalization on the flame resistance of polymer materials such as MPDI (Nomex), HBA/HNA (Vectra), and poly(ethylene terephthalate) (PET).

\section{Problem}

The combustion of polymeric materials is a major factor contributing to the deaths and injuries caused by fires. Hence, schemes to improve the flame resistance of polymers are of considerable interest. Here, we explore the utility of thermally-induced covalent cross linking of polymers to improve their combustion response. The hypothesis to be tested is that thermal cross linking will restrict the flow of molten polymers that would ordinarily continue to propagate a flame.

\section{Approach}

Recently, it has been suggested that $B C B$ functional groups help to improve the flammability of polymer materials (Marks, 1994). Here, we evaluate this hypothesis by synthesizing and characterizing the physical properties of copolymers containing $\mathrm{BCB}$ functional groups in the backbone of the molecule. This is accomplished by synthesizing copolymers with systematic variations in the amount of terephthalic acid (TA) substituted by the thermally crosslinkable analog XTA.

Past research in our laboratory has confirmed that XTA can be incorporated into polymers such as PPTA (Kevlar) without significantly changing established processing schemes or the morphology of the final polymer solid. The monomer undergoes an irreversible cross linking reaction at $-350^{\circ} \mathrm{C}$, but lies dormant during conventional solution or melt processing. Copolymers containing systematic variations in XTA content have now been prepared from MPDI (Nomex) and HBA/HNA (Vectra). The synthesis of PET-co-XTA is currently underway.

Thermal Gravimetric Analysis (TGA) of all XTA containing copolymers studied to date generally show a decreasing onset of thermal degradation temperature with increasing XTA content. Char contents at $800^{\circ} \mathrm{C}$, which have been correlated with Limited Oxygen Index values, increase with increasing XTA content (in HBA/HNA), and first increase, followed by a decrease in MPDI.

Scanning Electron Microscopy (SEM) and Transmission Electron Microscopy (TEM) have been used to examine the microstructure of the region near the flame surface. The formation, growth, and coalescence of voids were noted in the region underneath the flame surface. Studies are undenway to determine any systematic changes in the morphology of the polymer as a function of XTA content.

Future work will examine the physical properties and microstructure of PET-co-XTA polymers, and copolymers prepared from the multi functional BCB monomers BXTA, DXTA, and QXTA.

\section{Recent Results}

Martin, D. C., Spilman, G. E., Markoski, L. J., Jiang, T., and Pingel, E., "Flame Resistance of Benzocyclobutene (BCB) Functionalized Copolymers," submitted to the Society of Plastics Engineers.

Martin, D. C., "Synthesis, Processing, and Characterization of Thermally Crosslinkable Thermotropic Copolyesters," final report for the Summer Faculty Research Program, Phillips Laboratory, Edwards AFB, CA, 1995. 


\section{Synthesis, Characterization and Systematic Fire Safety Evaluation of High Volume and Specialty Hydrolytically Stable Phosphate Oxide Containing Polymeric Materials}

\author{
Principal Investigator \\ James E. McGrath \\ Department of Energy \\ Virginia Polytechnic Institute and State \\ University
}

\section{Sponsor}

National Institute of Standards and Technology

\section{Objective}

To investigate the influence of organically bound phosphorus and silicon on fire safety behavior of polymeric networks materials via cone calorimetry.

\section{Problem}

Background research has demonstrated that incorporation of phosphorus into poly(amide)s, epoxy networks, polyesters, and polycarbonates reduce the heat release rate, as judged by cone calorimetry, while maintaining a good balance of physical properties expected for the polymeric material. In general, these materials are self-extinguishing as judged by traditional tests, but cone calorimetry also shows that there is an increase in the carbon monoxide and smoke content of the phosphorus containing polymers relative to control materials which, undesirably burn rather quickly. The problem is to discern whether reduction of the aromatic content can maintain good improvement in heat release rate, while generating less smoke, etc. In addition, during this year, initial experiments have been conducted with siloxane containing block or segmented copolymers and/or curing agent/tougheners for epoxy networks.

\section{Approach}

Suitable monomers have been prepared for high performance copolymers, such as polymides, and poly(arylene ether)s. In particular, poly(arylene ether)s containing phosphorus have been initially screened by cone calorimetry and do show good behavior with respect to heat release rate and self-extinguishing characteristics. In the high performance class of polymide materials, modified poly(ether imide)s have been prepared which show excellent char yields in air and high temperatures in contrast to the control poly(ether imide)s. Aromatic phosphate oxide containing diamines were also used to cure epoxy resins and it was demonstrated that the reduction in heat release rate could be obtained. Initial efforts have been conducted with functional siloxanes as modifiers for polyester, polyamides, and epoxy networks. Initial aspects of the chemistry have been successful and preliminary polymerizations have been conducted demonstrating that the organosiloxane can effectively modify an epoxy network. Studies are underway that show the functional siloxane can also be introduced into engineering polyesters. Cone calorimetry results will be sought on these new materials as well as phosphorus systems.

\section{Recent Results}

Riley, D. J., Srinivasan, Gungor, A., Tchatchousa, C., Sandarapandian, M., and McGrath, J. E., "Synthesis and Characterization of Novel Flame Resistant Poly(arlene ethers)s," Polymer Preprints, Division of Polymer Chemistry, American Chemical Society, vol. 37, no. l, p. 489-490, 1996.

Tan, B., Tchatchoa, C. N., Vasudevan, V., Dong, L., and McGrath, J. E., "Phenyl Ethynyl Endcapped Arlene Ether Imide Oligomers/Polymers Containing Diaryl Alkyl Phosphine Oxide Groups," Polymeric Materials, Science and Engineering Preprints, vol. 74, 1996.

McGrath, J. E., Ghassemi, H., Riley, D., Bhatnager, A., and Kashiwagi, T., "The Synthesis and Characterization of New Thermoplastic Fire Resistant Materials," Society for Plastics Engineerings, ANTEC '96, Indiannapolis, IN, May 5-9, 1996.

Lee, Y. J., Gungor, A., Yoon, T. H., and McGrath, J. E., "Adhesive and ThermoMechanical Behavior of Phosphorus-Containing Thermoplastic Polymides," Adhesion, J., vol. 55, 
pp. 165-177, 1995.

Wan, I. Y., McGrath, J. E., and Kashiwagi, T.,

"Triarylphosphine Oxide Containing Nylon 6.6 Copolymers," ACS Symposium Series, No. 499,

"Fire and Polymers," Washington, DC, 1995.

Knauss, D. M., McGrath, J. E., and Kashiwagi, T., "Copolycarbonates and Poly(Arylates)

Derived from Hydrolytically Stable Phoshine

Oxide Comonomers," ACS Symposium Series, No. 499, Fire and Polymers, Washington, DC, 1995.
3. Modeling of Combustion, Fluid Mechanics, and Radiation in Buoyant Turbulent Fires

\section{Principal Investigator}

Ofodike A. Ezekoye

Department of Mechanical Engineering

The University of Texas at Austin

512-471-3085

\section{Sponsor}

National Institute of Standards and Technology

\section{Objective}

To evaluate and develop a computational strategy to couple gas phase combustion and soot radiation into simulations of buoyant flames.

\section{Problem}

Soot species radiation is the driving heat transfer mechanism for many fire scenarios. Computational approaches to describing the soot species evolution and subsequent radiative participation are very much in the developmental phase. Prior to adequately characterizing the radiative feedback and burning rate of a fuel bed, it is imperative to characterize the flame properties as to soot loading and radiative strength.

\section{Approach}

Lacking significant flow field effects, we have evaluated and refined models for soot driven radiative extinction (FY 1994), gas phase and soot transport, and soot oxidation and agglomeration processes (FY 1995). In these studies, combustion dynamics are computed for a spherical, transient, diffusion flame. Transformations of the conservation equation into a mixture fraction coordinate by means of Crocco type transformations, have made the choice of flow field effectively unimportant. The strain rate as is manifested in the scalar dissipation becomes the primary issue. Results generated for the spherical flames in question apply to any low strain rate diffusion flame when presented in mixture fraction space.

As with all flamelet strategies, the laminar nature of the flamelet implies that direct 
computation of the necessary combustion reactions can be performed at any level of detail. Thus, reaction chemistry can be specified to be either detailed or simplified. Various levels of chemical mechanism specification are investigated in this research.

An appropriate level of physical processes must be specified for the flamelet such that an appreciation and intuition for the overall dynamics present for a simulated large fire flow field will later be accurately modeled. This implies that both the soot species and temperature distributions are accurately computed initially on the flamelet.

Recent computations have produced good comparisons with experiments of a micro gravity diffusion flame. In these computations finite rate mechanisms are used for the gas and soot species evolution. In that study, it was found that the radiative modification of both the flame temperature field and the burner surface affected the soot production significantly. It also was found that oxidation of soot by the hydroxyl radical could significantly affect the soot species evolution. Finally, it was shown that the present spherical coagulation model used in most soot evolution models may lead to erroneous conclusions on the temporal evolution of the soot surface area.

\section{Recent Results}

Exekoye, O. A., and Zhang, Z., "Soot Oxidation and Agglomeration Modeling in a Micro gravity Diffusion Flame," Combustion and Flame, in review.

Zhang, Z., and Ezekoye, O. A., "Analysis of Laminar Acetylene-Air Diffusion Flames Using Reduced Reaction Mechanisms and State Relationships," Combustion Science and Technology, 1995.

Zhange, Z., and Ezekoye, O. A., "Acetylene-Air Diffusion Flame Computations; Comparisons of State Relations versus Finite Rate Kinetics," Proceedings of International Conference on Fire Research and Engineering, Orlando FL, 1995.

Ezekoye, O. A., and Zhang, Z., "Modeling of a Lagrangian Flamelet with Radiation

Interaction," Combustion Science and Technology,
106, 1995.

Zhang, Z., and Ezekoye, O. A., "Computational Study of State Relationships for Acetylene-Air Diffusion Flames with Soot Radiation," HTD bound vol. 304, ASME NHTC, Portland, OR August 1995. 


\section{Fire Growth Models for Materials}

Principle Investigator

Professor James Quintiere

Fire Protection Engineering

University of Maryland

301-405-3993

\section{Sponsor}

National Institute of Standards and Technology

\section{Objective}

To determine transient burning rate models that can be used to generalize data from the Cone Calorimeter.

\section{Problem}

Bench-scale test methods developed by BFRL's fire program have become standards, and offer the potential for scientifically based assessments of flammability. These test methods, based on the Cone Calorimeter and the LIFT apparatuses, provide material data on ignition, flame spread and burning or energy release rate for small samples. There is a need for using these data to describe the fire hazard of materials. The problem is to identify the form of these data consistent with general models for ignition, burning rate, and spread.

\section{Approach}

A model with good accuracy has been successfully demonstrated for thermoplastic like materials. This is based on an integral method that leads to a closed form analytical solution for constant heat flux. As a result, applications of that model will be examined. First, we have examined the case of step changes in irradiance for the thermoplastic case. Good agreement between the analytical solution and data for PMMA have been found. Second, we are examining the effect of transient burning rates on upward flame spread. We have developed an algorithm using the thermoplastic burning rate solution. The algorithm also accounts for the transient effect of the ignition flame, and the finite mass of the material which controls burnout. Third, we have extended the structure of the thermoplastic model to include the insulating effect of char accumulation. An algorithm for this charring case has been formulated to yield mass loss rate, char surface temperature, and char layer depth. Both algorithms are being coded so that quantitative results can be examined. Fourth, we are investigating the prospect of applying our fire growth simulation model to a large data base of commercial materials used in room corner fires. The simulation model would be upgraded to use the transient burning rate algorithms in development.

\section{Recent Results}

Quintiere, J., and Iqbal, N., "An Approximate Integral Model for the Burning Rate of a Thermoplastic-like Material," Fire and Materials, vol. 18, 89-98, 1994.

Iqbal, N., and Quintiere, J., "Flame Heat Fluxes in PMMA Pool Fires," SFPE Journal, vol. 6, no. 4, 153-162, 1994.

Quintiere, J., Hopkins Jr., D., Iqbal, N., and Rhodes, B., "Thermoplastic Pool Fires," The Symposium on Thermal Science and Engineering in Honor of Chancellor Chang-Lin Tien, November 14, 1995.

Rhodes, B. T., and Quintiere, J. G., "Burning Rate and Heat Flux for PMMA in the Cone Calorimeter," Fire Safety Journal, September 1995.

Hopkins Jr., D., "Predicting the Ignition Time and Burning of Thermoplastics in the Cone Calorimeter," MS Thesis, Department of Fire Protection, U. of MD, May 1995.

Hopkins Jr., D., and Quintiere, J. G., "Material Fire Properties and Predictions for Thermoplastics," submitted to the Fire Safety Journal, December 1995.

Quintiere, J. G., "Fire growth: a review," submitted to the Philosophical Transactions of the Royal Society, April 1995.

Quintiere, J. G., Hopkins Jr., D., and Hopkins, M., "Room -Corner Fire Prediction for Textile Wall Materials," 1995 International Conference on Fire Research and Engineering, September, 1995. 



\section{FIRE SCIENCE DIVISION}

SMOKE DYNAMICS

RESEARCH 



\section{Carbon Monoxide Production and Prediction}

\section{Principal Investigator \\ William M. Pitts \\ Fire Science Division \\ 301-975-6486}

\section{Sponsor}

National Institute of Standards and Technology

\section{Objective}

To develop a fundamental understanding of the mechanisms of carbon monoxide formation in flames sufficient to produce an estimation and detailed predictive models.

\section{Problem}

$\mathrm{CO}$ generated by fires in enclosures is responsible for roughly two-thirds of fire deaths. The conditions necessary and the mechanisms responsible for the generation of high concentrations of $\mathrm{CO}$ are poorly characterized. As a result, predicting the generation of $\mathrm{CO}$ by fires is currently impossible. Laboratory fire models require such a predictive capability such as BFRL's HAZARD Fire Model.

\section{Approach}

During FY 1996, BFRL will perform fundamental and engineering investigations to develop appropriate correlations of $\mathrm{CO}$ formation for fires in enclosures and to provide the necessary knowledge to incorporate the findings into existing BFRL fire models. The fundamental investigations will identify the principal chemical and physical mechanisms responsible for the formation of high $\mathrm{CO}$ concentrations in fires and to theoretically justify the use of the engineering correlations. Ultimately, the findings will be incorporated into a simple physical model for $\mathrm{CO}$ production in enclosure fires.

\section{Recent Results}

Pitts, W. M., "An Algorithm for Predicting Carbon Monoxide Formation in Enclosure Fires," submitted for the Fifth International Symposium on Fire Safety Science, March 3-7, 1997.
Johnsson, E. L., Bryner, N. P., and Pitts, W. M., "Fire-Induced Mass Flow into a Reduces-Scale Enclosure," Fire Safety Journal, in press.

Pitts, W. M., “The Global Equivalence Ratio Concept and the Formation Mechanisms of Carbon Monoxide in Enclosure Fires," Progress in Energy and Combustion Science, vol. 21, p. 197, 1995.

Pitts, W. M., The Global Equivalence Ratio Concept and the Prediction of Carbon Monoxide Formation in Enclosure Fires, NIST Monograph 179, National Institute of Standards and Technology, June 1994.

Pitts, W. M., "Application of Thermodynamic and Detailed Chemical Kinetic Modeling to Understanding Combustion Product Generation in Enclosure Fires," Fire Safety Journal, vol. 23, p. $271,1994$.

Pitts, W. M., Johnsson, E. L., and Bryner, N. P., "Carbon Monoxide Formation in Fires by HighTemperature Anaerobic Wood Pyrolysis,"

Twenty-Fifth International Symposium on Combustion, The Combustion Institute, pp. 14551462, Pittsburgh, PA, 1994.

Leonard, S., Mulholland, G., Puri, R., and Santoro, R. J., "Generation of CO and Smoke During Underventilated Combustion," Combustion and Flame, vol. 98, p. 20, 1994. 


\section{Species Production for Turbulent Flame Modeling}

Principal Investigators

Kermit C. Smyth

Fire Science Division

301-975-6490

George W. Mulholland

Fire Science Division

301-975-2660

Nelson Bryner

Fire Science Division

301-975-6868

\section{Sponsor}

National Institute of Standards and Technology

\section{Objective}

To develop an accurate data base and phenomenological descriptions to establish models for in-flame species concentrations and post-flame species yields as a function of residence time, equivalence ratio, temperature, and strain and scalar dissipation rates.

\section{Problem}

The data base, new metrology, phenomenology, and models for well-controlled flame environments will provide the foundation for predicting flame radiation and the production of toxic gases and smoke for buoyant, turbulent diffusion flames for a wide range of combustion conditions.

\section{Approach}

During FY 1996, BFRL will select and experimentally examine laminar flame environments with temperature, residence time, equivalence ratio, and strain and scalar dissipation rates characteristic of buoyant, turbulent flames. Emphasis will be placed on planar imaging approaches using laser-induced fluorescence and scattering signatures, and quantitative measures of the soot field. Initial studies will focus on time-varying and underventilated laminar diffusion flames. Characterize the in-flame and post-flame chemical species, flow velocity, and temperature fields. Apply GC-MS and laser ionization-mass spectroscopy (previously used for graphitic smoke) to the analysis of the mostly organic smoke produced during underventilated combustion. Test and develop models incorporating the wide range of combustion conditions using the new data base.

\section{Recent Results}

Shaddix, C. R, and Smyth, K C., "Laser-

Induced Incandescence Measurements of Soot

Production in Steady and Flickering Methane, Propane, and Ehtylene Diffusion Flames,"

Combustion and Flames, in press.

Kaplan, C. R, Shaddix, C. R, and Smyth, K C., "Computations of Enhanced Soot Production in Time-Varying $\mathrm{CH}_{4} /$ Air Diffusion Flames," Combustion and Flame, in press. 
Vortex-Chemistry Interactions in Methane/Air Diffusion Flames

\author{
Principal Investigators \\ Kermit C. Smyth \\ Fire Science Division \\ 301-975-6490
}

William M. Pitts

Fire Science Division

301-975-6486

\section{Sponsor}

Gas Research Institute

\section{Objective}

To determine how chemical production and destruction rate for soot, $\mathrm{CO}$, and NO depend upon local flame conditions.

Investigate a wide range of combinations of the key parameters, specifically residence time, mixture fraction, temperature, and strain and scalar dissipation rates.

\section{Problem}

Increasingly tighter regulations from the Clean Air Act and its 1990 amendments now control pollutant emissions. In cases where the chemical kinetics is well established, e.g., CO production and destruction, emission levels cannot be predicted from practical combustion devices over a range of operating conditions.

\section{Approach}

During FY 1996, BFRL will study chemistryflowfield interactions using flickering methane/air diffusion flames. Axisymmetric flames will be acoustically locked to a pulsed dye laser system at $10 \mathrm{~Hz}$, allowing interrogation of the flame dynamics as a function of height, phase angle, and forcing amplitude. These flames exhibit a much wider range of local strain and scalar dissipation rates than those found in steady, co-flowing laminar flames. Emphasis will be placed on planar imaging approaches for species and soot concentrations, and LDV velocity and thinfilament pyrometry temperature measurements. These results will provide critical tests of current models for the production of toxic gases and smoke from buoyant, turbulent diffusion flames for a wide range of combustion conditions.

\section{Recent Results}

Smyth, K. C., "NO Production and Destruction in a Methane/Air Diffusion Flame," Combustion Science and Technology, 115: 173-194, 1996.

Everest, D. A., Shaddix, C. R., and Smyth, K C., "Quantitative Two-Photon Laser-Induced Fluorescence Imaging of $\mathrm{CO}$ in Flickering $\mathrm{CH}_{4}$ /Air Diffusion Flames," Twenty-Sixth International Symposium on Combustion, April 1996.

Shaddix, C. R, Harrington, J. E., and Smyth, K. C., "Quantitative Measurements of Enhanced Soot Production in Flickering Methane/Air Diffusion Flame," Combustion and Flame, vol. 99, p. 723,1994 , comment and reply Combustion and Flame, vol. 100, p. 518, 1995. 


\section{RECENT GRANTS - SMOKE DYNAMICS RESEARCH}

\section{Mixing and Radiation Properties of Buoyant Luminous Flame Environments}

\author{
Principal Investigator \\ G.M. Faeth \\ Department of Aerospace Engineering \\ The University of Michigan \\ 313-764-7202
}

\section{Sponsor}

National Institute of Standards and Technology

\section{Objective}

To complete measurements of the radiation and mixing properties of luminous buoyant turbulent flame environments, and to exploit these results to develop and evaluate advanced models of practical unwanted fires.

\section{Problem}

Radiation and turbulent mixing from luminous flames strongly affect the rates of spread, growth and combustion of unwanted fires; therefore, these properties are important elements of contemporary models of unwanted fires that find application for quantifying fire hazards while avoiding the time and cost of fullscale fire tests. Unfortunately, available information about soot optical properties are limited although radiation from soot in luminous flames is the dominant radiation mechanism of fires. In addition, the fundamental transport and mixing properties of the buoyant turbulent flows found in fire environments are poorly understood which has impeded the development and evaluation of fire models. Thus, both issues are being addressed during this investigation.

\section{Approach}

The study of the radiation properties of luminous flames is concentrating on the optical properties of soot because continuum radiation from soot is the most important source of radiation in fires. Measurements and computations during initial phases of the study showed that soot optical properties can be approximated by the Rayleigh-Debye-Gans (RDG) scattering approximation while treating soot as polydisperse mass-fractal aggregates of primary soot particles. Measurements also revealed that the mass-fractal properties of soot aggregates were robust; therefore, current large uncertainties about the refractive indices of soot, and how they vary with fuel type and flame conditions, are the greatest impediments to reliable estimates of soot optical properties.

In view of these findings, current work is concentrating on soot refractive indices. The first phase of this work involved in situ extinction and scattering measurements in the visible ( $350 \mathrm{~nm}$ to $800 \mathrm{~nm}$ ), combined with gravimetric measurements of soot densities and volume fractions, to yield soot refractive indices for this wavelength range. Measurements thus far have considered soot emitted from gasfueled buoyant turbulent diffusion flames in the long residence time regime where soot properties are independent of position in the overfire region and residence time; it was found that the refractive indices of this soot were relatively independent of fuel type and agreed reasonably well with the often criticized classical measurements of soot refractive indices of Dalzell and Sarofim. Current work is undertaking similar measurements for more heavily sooting liquid-fueled diffusion flames, and is developing ex situ reflectivity measurements needed to address refractive indices in the infrared wavelength range. Subsequent work will consider other types of soot found in fire environments and will evaluate radiation predictions for sootcontaining luminous flames using present methods, based on radiation measurements for well-characterized luminous laminar diffusion flames.

The study of buoyant turbulent flows is concentrating on self-preserving buoyant turbulent plumes because they are fundamental classical flows that are independent of source properties and can be numerically simulated with modest computational requirements. Present work began with self-preserving round buoyant turbulent plumes using laser-induced fluorescence and laser velocimetry to measure 
mixing and velocity properties. These

measurements showed that earlier work did not extend far enough from the source to reach selfpreserving conditions, and that self-preserving round turbulent plumes were narrower, and had larger scaled mean values near the axis, than previously thought. These observations raised questions about potential effects of flow confinement during these experiments, because ambient coflows due to confinement would also tend to narrow the plumes; nevertheless, new experiments addressing these effects showed that coflows were not a factor and that present results were properly the outcome of fully reaching self-preserving conditions. Attention then turned to measuring turbulence properties in the self-preserving region and comparing these measurements with predictions based on contemporary turbulence models; the results highlighted several deficiencies of present models for treating buoyant turbulent flows typical of fire environments and provided information needed to develop more effective methods.

Attention has now turned to the classical problem of self-preserving plane (or line) buoyant turbulent plumes for several reasons. First, the streamwise rates of development of self-preserving round and line buoyant turbulent plumes are fundamentally different which provides a useful evaluation of modeling improvements needed to treat the complex flows of practical fire environments. Secondly, the line buoyant turbulent plume is a necessary precursor for gaining a better understanding of turbulent flows along surfaces that are relevant to burning objects. Finally, reaching selfpreserving flow conditions is more difficult for line than round plumes; therefore, concerns about existing measurements that claim to represent self-preserving behavior must be resolved before this information can be exploited for model development. Current work is developing the new line-plume apparatus, with measurements of mixing and velocity properties to follow. Subsequent work will address the effectiveness of existing turbulence models, based on evaluations using present measurements in the self-preserving region of line plumes.

\section{Recent Results}

Farias, T. L., Carvalho, M. G., Koylu, U. O., and Faeth, G. M., "Computational Evaluation of Approximate Rayleigh-Deby-Gans/FractalAggregate Theory for the Absorption and Scattering Properties of Soot," J. Heat Trans., 117, 152, 1995.

Koylu, U. O., Faeth, G. M., Farias, T. L., and Carvalo, M. G., "Fractal and Projected

Structure Properties of Soot Aggregates,"

Combust. Flame, 100, 62 1, 1995.

Faeth, G. M., and Koylu, U. O., "Soot Morphology and Optical Properties in Nonpremixed Turbulent Flame Environments," Combust. Sci. Tech., 108, 207, 1995.

Dai, Z., Tseng, L. K. and Faeth, G. M., "Velocity/Mixture-Fraction Statistics of Round Self-Preserving Buoyant Turbulent Plumes," J. Heat Trans., 1 17, 918, 1995. 


\section{Dynamics, Transport and Chemical Kinetics of Compartment Fire Exhaust Gases}

\author{
Principal Investigators \\ Uri Vandsburger \\ Virginia Polytechnic Institute and State \\ University \\ 703-231-4459
}

Richard J. Roby

Hughes Associates Inc.

410-737-8677

\section{Objective}

The study focuses on determining the conditions for the transport of high concentrations of $\mathrm{CO}$ to remote locations from the burning compartment, and the transport path of $\mathrm{CO}$ away from the compartment.

\section{Problem}

Over the past decade, the percentage of deaths from smoke inhalation in building fires has risen by $1 \%$ every year, and was responsible for $75 \%$ of the deaths during 1992. Existing engineering models need to be improved to accurately predict the amount of $\mathrm{CO}$ transported under certain conditions and the path of the $\mathrm{CO}$ transport.

\section{Approach}

The reduced-scale building fire facility at VPI\&SU was arranged with the compartment $(1.22 \mathrm{~m}$ by $1.52 \mathrm{~m}$ by $1.22 \mathrm{~m})$, which contained a liquid hexane pool fire, placed on the side at the end of the hallway $(1.67 \mathrm{~m}$ by $1.22 \mathrm{~m}$ by $5.18 \mathrm{~m})$ forming a L-shape. The global equivalence ratio of the compartment fires was $3.0 \pm 0.3$ during the post-flashover period.

The oxygen entrainment into the compartment fire gas plume entering the hallway controls the degree of $\mathrm{CO}$ oxidation. The size of the opening connecting the compartment and hallway, the height of the soffit at the opening and the hallway upper-layer depth (controlled by the height of the soffit at the end of the hallway) all play a role in the entrainment of oxygen into the plume.
Through varying the upper-layer depth, three classes of hallways were defined: "semi-infinitely long," "long," and "short." The "semi-infinitely long" long hallway characteristically had a shallow upper-layer $(0.0 \mathrm{~m}$ to $0.20 \mathrm{~m})$, and the downstream yield of $\mathrm{CO}$ was at a minimum with its magnitude controlled by the opening size. A $0.04 \mathrm{~m}^{2}$ opening resulted in lower $\mathrm{CO}$ yields $(0.02)$ compared with a $0.12 \mathrm{~m}^{2}$ opening (0.10). The "long" hallway, which had a medium upper-layer depth $(0.20 \mathrm{~m}$ to $0.60 \mathrm{~m})$, generated yields which were a transition between the low yields of the "semi-infinitely long" hallway to the high yields of the "short" hallway. The deep upper-layer $(0.60 \mathrm{~m}$ and greater) in the "short" hallway resulted in $\mathrm{CO}$ yields equivalent to or higher than those measured inside the compartment (0.22), and were independent of the opening size. The higher yields were observed in experiments with external burning where unburned hydrocarbons (UHC) were oxidized while $\mathrm{CO}$ was not.

With the compartment on the side of the hallway, the flow field in the hallway is highly three-dimensional resulting in a non-uniform transport of gases down the hallway. During the compartment post-flashover period of "short" and "long" hallway experiments with external burning, gases containing high levels of $\mathrm{CO}(2.0 \%$ to $2.5 \%)$ first travel across the hallway. Gases measured containing 1.8 percent to 2.0 percent $\mathrm{CO}$ were transported down the side of the hallway opposite the compartment, while only $0.8 \% \mathrm{CO}$ was present on the compartment side of the hallway. The non-uniformity persisted in the "long" hallway experiments for the entire post-flashover period (140 seconds), while the upper-layer CO concentration became uniform in "short" hallway experiments 70 seconds after flashover. The UHC were oxidized to 1.0 percent after the gases had traveled across the hallway. Based on these observations, the Hillhaven nursing home fire in 1991 was an example of a long hallway. Of the 23 people residing along the hallway, 11 people died on the side of the hallway opposite the burning room, while only 1 person died on the burning room side of the hallway.

The zonal models currently used in fire safety codes, which assume uniform upper-layer 
species concentrations, are valid in cases with a short hallway or at times long after the onset of flashover. Instead, field models should be used to accurately model smoke and toxic gas movement in hallways with the burning room located on the side. Future experiments will be performed with different fuels (e.g.,

polyurethane foam), and door connecting the compartment to the hallway. Computer modeling will be conducted to verify the experimental results.

\section{Recent Results}

Vandsburger, U., Lattimer, B.Y., and Roby, R.J., Compartment Fire Combustion Dynamics, Annual Report, NIST-GCR-95-666, National Institute of Standards and Technology, 1994. 



\section{BFRL HEADQUARTERS}

OFFICE OF APPLIED

ECONOMICS 



\section{Economic Support for the NIST Advanced Technology Program}

\section{Principal Investigator}

Stephen F. Weber

Office of Applied Economics

301-975-6137

\section{Sponsor}

National Institute of Standards and Technology Advanced Technology Program (ATP)

\section{Objective}

To support the economic and program evaluation missions of ATP by providing economic modeling services and by designing and developing economic questionnaires and implementing them in sof tware to permit electronic submission by ATP awardees of quarterly and annual reports on economic and business progress.

\section{Problem}

ATP management is developing questionnaires to track the technological and economic progress of awardees. Economic consulting is needed to review the questions, and sof tware is needed to implement them in electronic form. ATP also needs assistance in conducting economic impact assessments of its projects.

\section{Approach}

During FY 1996, BFRL will support ATP by reviewing the questionnaires for their economic content and working with ATP economists to specify data requirements and to design efficient ATP awardee questionnaires to support economic impact models. OAE will develop data structures, files, and data relations for field data collection and storing questionnaire responses in a centralized database at ATP headquarters. OAE will computerize in separate, executable, software modules each of the six ATP awardee questionnaires. Some data validation features and help screens will be included. OAE also will develop software utilities for creating distribution diskettes and for consolidating data from floppy disks into a centralized database at ATP headquarters.

OAE will work with ATP economists to develop methods to estimate economic impacts of ATP funded research and apply econometric forecasting and simulation tools to ATP case study projects.

\section{Results}

Enhanced software modules for ATP's quarterly report on Business Plan and Developments.

Completed software for ATP's anniversary report on Economic and Financial Data. 


\section{Measurement of BFRL Research Impacts}

\section{Principal Investigator}

Robert Chapman

Office of Applied Economics

301-975-2723

\section{Sponsor}

National Institute of Standards and Technology

\section{Objective}

To help BFRL identify industries impacted by BFRL research, estimate economic impacts resulting from BFRL research, and estimate the return on BFRL's research investment dollars.

\section{Problem}

The measurement of economic impacts of research is a major objective of BFRL.

Managers need to know the impact of their research programs to achieve the maximum social benefits from their limited budgets.

Improved methods for measuring economic impacts is essential to BFRL to help select the "best" among competing research programs, to evaluate how cost effective are existing research programs, and to defend or stop programs based on their economic impact.

\section{Approach}

During FY 1996, BFRL staff will be developing standard methods for measuring economic impacts of research projects. The focus is on measures of net benefits, the benefit-to-cost ratio, and the social rate of return. These methods are consistent with ASTM methods of economic evaluation.

A classification of typical benefits and costs associated with scientific research is being established to help researchers identify specific benefits and costs of their research. The initial classification will focus on building-related research projects. This will help researchers and managers think in economic impact terms when planning projects and when evaluating them expost.

Three summary economic impact studies performed in FY 1995 will be expanded and published as two separate reports. The three studies included:

- The substitution of 235 for 210 pound shingles in asphalt roofing shingles,

- Improving residential energy conservation according to ASHRAE 90-75,

- Using the Fire Safety Evaluation System (FSES) to find the least-cost compliance with fire-safety performance codes in health care occupancies.

One report will cover the first two buildingrelated studies; the second report will cover the last study.

In subsequent years, BFRL staff will develop techniques for measuring impacts for specific categories of customers (e.g., by SIC codes). The objective will be to produce the database and decision support software products that will enable BFRL to identify its customers by SIC categories, measure the customers' contributions to GDP, measure the impact of BFRL's research on GDP and employment by region of the U.S., and to evaluate and measure the impact of new research opportunities to determine those with the greatest likely national and regional economic impact.

\section{Recent Results}

Chapman, Robert and Fuller, Sieglinda, Benefits and Cost of Research: Two Case Studies in Building Technology, NISTIR 5840, National Institute of Standards and Technology, 1996.

Chapman, Robert and Weber, Stephen, Benefits and Costs of Research: A Case Study of the Fire Safety Evaluation System, NISTIR 5863, National Institute of Standards and Technology, 1996. 


\section{Economics of New-Technology Materials}

\author{
Principal Investigator \\ Mark Ehlen \\ Office of Applied Economics \\ 301-975-4522
}

\section{Sponsor}

National Institute of Standards and Technology

\section{Objective}

To develop life-cycle economic methods for evaluating the economics of substituting new materials, such as composites or highperformance concrete and steel, for traditional materials in civil engineering applications.

\section{Problem}

The construction industry needs sophisticated, practical methods and guidelines for evaluating alternative building materials in a consistent manner over their life cycle. The need for standardized, improved methods and guidelines stems in part because high up-front costs of new-technology construction materials discourage their application even when warranted by life-cycle criteria. Providing a method for evaluating life-cycle cost effectiveness will give decision makers a tool to help them select, both for research and applications on the job, those materials that will deliver the nation's infrastructure at least cost.

\section{Approach}

During FY 1996, staff will develop and publish a report that will help analysts evaluate the cost effectiveness of new materials in typical construction applications such as bridge building. A case study will evaluate composites in comparison to conventional materials in the construction of bridge decks. An initial prototype of decision-support software will be prepared to help state and Federal Department of Transportation officials choose cost-effective materials when issuing construction specifications for the construction and repair of bridges. To disseminate the evaluation method presented in the report, at least one technical presentation will be given to potential users and a technical article will be prepared for a refereed journal.

In subsequent years, additional methods, such as the adjusted internal rate of return and net savings methods, will be developed. Techniques for taking into consideration economic uncertainty and risk will be explored. A multicriteria decision method that allows users to simultaneously evaluate multiple economic and engineering criteria when choosing among alternative materials also will be developed. Through participation in the ASTM Building Economics Subcommittee (E06.81), efforts will be made to have these methods and techniques consistent with existing and future ASTM building economics standards. Case studies using these methods and techniques will be prepared for both new (e.g., high-performance concrete) and conventional materials competing for use in bridge decks and in other applications such as high-performance building systems. Decision support software will be developed to implement the methods and techniques for multiple construction applications.

This three-year project supports the CONMAT Implementation Plan concerning Life-Cycle Cost Analysis. It also supports the National Construction Goals by promoting reductions in project delivery time and providing enhanced durability.

\section{Recent Results}

Ehlen, Mark and Marshall, Harold, The

Economics of New-Technology Materials in

Construction: A Case Study of FRP Composite

Bridge Decking, NISTIR 5864, National Institute of Standards and Technology, 1996. 


\section{Optimal Pricing of Standard Reference Materials}

Principal Investigator

Sieglinde K. Fuller

Office of Applied Economics

301-975-6134

\section{Sponsor}

National Institute of Standards and Technology

\section{Objective}

To provide the Standard Reference Materials Program (SRMP) with an optimal pricing model, based on an analysis of demand for SRMs, and subject to the constraint of having to cover the cost of the program.

\section{Problem}

Since the early 1980's, NIST, like other government agencies, has been increasingly under pressure to recover the total cost of their services through user fees rather than through tax appropriations. The required changes in the pricing policies of the SRMP have resulted in higher prices for SRMs. From the observed changes in aggregate unit sales and total revenues it is not clear whether or how the price changes have affected the demand for SRMs, given that other factors may affect demand. To formulate pricing rules that cover the cost of the program and do not adversely affect sales of SRMs, it is imperative to include demand-side information besides cost and supply considerations.

\section{Approach}

During FY 1996, BFRL will perform four tasks:

1. Explain the theoretical model and its relationship to intermediate goods such as NIST SRMs.

2. Derive the optimal pricing formula for SRMs.

3. Test the model with two SRMs to develop the algorithms for subsequently implementing the model for the 24 SRMs for which the demand analyses have been completed.

4. Draft a report describing the SRM model and the results of the two-SRM test.
Public-sector economic theory provides an operative optimization model for calculating welfare-maximizing prices and outputs for government enterprises that supply multiple goods and services to users that can be identified and charged fees. This model will be adapted to intermediate goods and implemented for SRMs. As the first step, demand functions were derived for a selected group of SRMs. The resulting price elasticities of demand, which estimate the response of unit sales to price changes, will be combined with cost data to determine optimal prices and saleable quantities. The research will estimate the welfare gain from the optimal pricing rule compared with alternative pricing formulas and make recommendations to support SRMP pricing policy in funding and sales negotiations.

\section{Recent Results}

In FY 1995 demand functions and price elasticities were developed for 24 SRMs. 


\section{Baseline Measures for National Construction Goals}

\section{Principal Investigator \\ Robert Chapman}

Office of Applied Economics

301-975-2723

\section{Sponsor}

National Institute of Standards and Technology

\section{Objective}

To support the Subcommittee on Construction and Building (C\&B), a part of the National Science and Technology Council, identify baseline measurement data of current practice with respect to two committee thrusts important to advancing the U.S. construction industry: 1. improving the life cycle of constructed facilities; and 2. improving health and safety of the construction workforce.

\section{Problem}

The two priority thrusts specify the following goals to be attained by the year 2002 :

\section{Better constructed facilities:}

- 50 percent reduction in delivery time

- 50 percent reduction in operation and maintenance

- 30 percent increase in productivity and comfort

- 50 percent fewer occupant-related illnesses and injuries

- 50 percent less waste and pollution

- 50 percent more durability and flexibility

\section{Health and Safety of the Construction Workforce}

- 50 percent reduction in related illnesses and injuries.

Data describing current practices of U.S. firms with respect to the identified goals (e.g., 50\% reduction in delivery time) are needed to establish a baseline against which to measure progress of the construction industry toward achieving these goals. It is essential to have baseline data and associated measures of progress toward improved performance to determine the success of actions taken to improve the competitiveness of the construction industry in U.S. and international markets.

Having these measures of progress will make it possible to demonstrate the benefits of advanced technologies and practices.

\section{Approach}

During FY 1996, BFRL staff will collect data on current industry performance of the seven listed goals. This work will be part of a collaborative effort including other government agencies such as the Bureau of the Census and the Occupational Safety and Health Administration, private-sector organizations, and academic institutions. These data on industry performance will become the baselines for measuring progress toward the achievement of the identified percent reduction goals. The data will be delivered initially as tables and reports. They will be ultimately expanded into computerized delivery systems available on the Internet.

A start will be made this year on developing methods for measuring progress. In subsequent years these methods will be applied to the measurement of progress toward the seven above goals. The methods will be made available on the Internet. User-friendly decision support software also will be developed to access baseline data and to measure progress toward goals.

\section{Recent Results}

New project 


\section{Building for Environmental and Economic Sustainability (BEES)}

\author{
Principal Investigator \\ Barbara C. Lippiatt \\ Office of Applied Economics \\ 301-975-6133
}

\section{Sponsor}

National Institute of Standards and Technology

\section{Objective}

To develop decision-support software for selecting building materials that achieve the most appropriate balance between life-cycle environmental and economic performance (second year of a 3-year project).

\section{Problem}

Environmental and economic performances are two key factors in building material selection decisions. The environmental performance of building materials, however, is not readily assessable and comparable to economic performance. A methodology is needed for assessing and comparing environmental against economic performance. To maximize technology transfer, the methodology needs to be implemented in publicly-available, user-friendly, decisionsupport software.

\section{Approach}

During FY 1996, BFRL staff will continue developing the methodology for assessing and comparing environmental against economic performance. It is assessing the environmental performance of building materials based on the concept of life-cycle assessment, an analysis of environmental impacts from raw materials extraction to ultimate material reuse, recycling, or disposal. BFRL contracted for collection of environmental performance data for five building materials for the BEES system (Building for Environmental and Economic Sustainability). BFRL will continue to assess economic performance using the life-cycle costing concept, and collect life-cycle cost data from the five materials. An Alpha version of the BEES system is being developed.

In FY 1997, BFRL will add five more building materials to the BEES systems and conduct a Beta test of the BEES software. The Beta test comments will be incorporated in a final review version of the BEES software and user manual. Finally, BFRL will continue its active participation in ASTM E50.06 (Green Buildings), and draft an ASTM document for ASTM E06.81 (Building Economics) illustrating how Multi-Attribute Decision Analysis can be used in life-cycle assessment.

\section{Recent Results}

Lippiatt, B.C. and Norris, G.A., "Selecting Environmentally and Economically Balanced Building Materials," Second International Green Building Conference and Exposition -- 1995, NIST Special Publication 888, National Institute of Standards and Technology, pp. 38-46, 1995.

Lippiatt, B.C. and Norris, G.A., "Selecting Environmentally and Economically Balanced Building Materials," reprinted in, Sustainable Building Technical Manual: Green Building Design, Construction, and Operations, Florida A\&M University, 1996. 


\section{Life-Cycle Costing Methodology}

\section{Principal Investigator}

Stephen R Petersen

Office of Applied Economics

301-975-6136

\section{Sponsor}

National Institute of Standards and Technology

U.S. Department of Energy

Federal Energy management Program

\section{Objective}

To provide on a continuing basis, economic analysis methods, data, software, training, and consulting to the DOE's Federal Energy Management Program in support of energy and water conservation projects in federal buildings and facilities.

\section{Problem}

The National Energy Conservation Policy Act directs NIST to provide expert consulting to DOE related to economic analysis of energy conservation projects in federal buildings. Executive Order 12902 of 8 March 1994, requires that energy usage in federal facilities be reduced by 30 percent by the year 2005 compared with 1985 levels, in a cost-effective manner.

\section{Approach}

During FY 1996, BFRL will provide, on a continuing basis, economic analysis methods, data, software, training, and consulting. BFRL is responsible for assisting DOE develop and modify FEMP LCC rules as promulgated in 10 CFR 436 Subpart A. BFRL is amplifying these rules and procedures in NIST Handbook 135, Life-Cycle Costing Manual for the Federal Energy Management Program.

Annually, BFRL computes the discount rate to be used in the analysis of federal energy and water conservation projects, using the procedure specified in 10 CFR 436. In addition, staff compute tables of discount factors and energy price indices based on this discount rate and on energy price projections received from DOE/EIA. These data are published each
October 1 in the Annual Supplement to Handbook 135 , and included in electronic form with BFRL's LCC software.

BFRL developed five computer programs for economic analysis of energy and water conservation projects: BLCC, Quick Input, DISCOUNT, ERATES, and EMISS. These programs are maintained, updated, and enhanced with new analytical capabilities annually. DOE and private-sector software vendors distribute these programs. User manuals are provided with each program. The sof tware also has been incorporated into several other software projects outside NIST, sponsored by DOE.

BFRL developed a basic LCC workshop which it has conducted five times each year at locations throughout the United States, primarily for federal engineers and energy managers, but attended by representatives from state and local government, utilities, and consultants from the private sector. In FY 1996, staff will be developing and teaching an advanced LCC workshop that is more oriented toward project analysis and the OAE LCC software. BFRL has trained 22 instructors, mostly university professors and private consultants, to conduct the basic LCC workshop, and is providing them required teaching materials. These trainers are now conducting many additional workshops throughout the United States, greatly expanding the reach of BFRL research activities. Staff have developed three LCC training videos with workbooks to promote the use of economic analysis in project design and maintenance.

BFRL provides consulting to DOE related to methods of economic analysis for federal energy and water conservation projects on a continuous basis. For example, changes to the FEMP LCC rules in 10 CFR 436 are made in consultation with OAE; users of BFRL publications and software frequently call with technical questions related to these materials and to interpretation of 10 CFR 436; and Staff are working with FEMP to reconcile differences in DOD's LCC methodology with the FEMP methodology used by other federal agencies. 


\section{Recent Results}

Fuller, Sieglinda, and Petersen, Stephen, LifeCycle Costing Manual for the Federal Energy

Management Program, NIST Handbook 135,

National Institute of Standards and

Technology, February 1996.

Petersen, Stephen R, BLCC-- The NIST

"Building Life-Cycle Cost" Program, Version 4.3

User's Guide and Reference Manual, NISTIR

5185-3, National Institute of Standards and

Technology, October 1995.

Petersen, Stephen R, EMISS-- A Program for Estimating Local Air Pollution Emission Factors Related to Energy Use in Buildings User's Guide and Reference Manual, NISTIR 5704, National Institute of Standards and Technology, October 1995.

Fuller, Sieglinde K, and Petersen, Stephen R, Life-Cycle Costing Workshop for Energy Conservation in Buildings: Student Manual, NISTIR 5165 ,

National Institute of Standards and

Technology, October 1995

Petersen, Stephen R, Energy Price Indices and Discount Factors for Life-Cycle Cost Analysis 1996: Annual Supplement to NIST Handbook 135 and NBS Special Publications 709, NISTIR 85-3273-

10, National Institute of Standards and

Technology, October 1995

Petersen, Stephen R, Present Worth Factors for Life-Cycle Cost Studies in the Department of Defense (1996), NISTIR 4942-3, National Institute of Standards and Technology, October 1995.

\section{Provide Economic Support to NIST Manufacturing Extension Partnership}

\author{
Principal Investigator \\ Stephen F. Weber \\ Office of Applied Economics \\ 301-975-6137
}

\section{Sponsor}

National Institute of Standards and Technology

\section{Objective}

To support the economic and program evaluation missions of MEP by identifying economic data sources, developing economic data analysis software, developing economic maps, and modeling economic impacts.

\section{Problem}

MEP management needs economic consulting support for planning, reporting, and program evaluation.

\section{Approach}

During FY 1996, BFRL will assist the NIST Manufacturing Engineering Program (MEP) in developing survey questions to capture data on the direct economic impacts at the individual establishment level resulting from Technical Assistance Projects (TAPs) of Manufacturing Extension Centers (MECs). BFRL will develop methods and obtain supporting data to derive industry level shock variables compatible with the REMI economic impact simulation model. BFRL will obtain preliminary data from MEP and conduct test analyses of MEP impacts using REMI model. Once sufficient survey data are available, staff will conduct REMI analysis and interpret the results.

Staff will also implement the application of Data Envelopment Analysis (DEA) to measure and suggest improvements in the relative efficiency of MECs. BFRL will work with MEP to identify the best input, output, and control variables to use in the DEA analysis. Staff will complete a working version of the DEA Excel software. Once data are collected by MEP, staff will apply the DEA software to the data and 
report the results to $\mathrm{MEP}$.

BFRL also will support MEP by providing economic data and maps to define the scope, scale, and geographic distribution of manufacturing establishments and Manufacturing Extension Centers (MECs).

\section{Recent Results}

Identified and successfully procured REMI economic forecasting and simulation software.

Developed prototype Excel programs to test the application of DEA analysis.

Developed economic maps showing small manufacturing industry concentrations and the coverage by county of all MTCs in the MEP system.

\section{Economic Support for the Office of Law Enforcement Standards}

\section{Principal Investigator}

Stephen F. Weber

Office of Applied Economics

301-975-6137

\section{Sponsor}

National Institute of Standards and Technology

\section{Objective}

To update and publish AutoBid software to support police vehicle acquisition decisions.

\section{Problem}

AutoBid software that supports police vehicle acquisition decisions needs to be updated to include the latest 1996 model year data on police vehicle performance. The current version of AutoBid is directly dependent on a specific set of data files that each cover one vehicle model year. The data files contain the performance test results for police patrol vehicles published annually by the Michigan State Police. The specific data files expected to be present are explicitly referenced in the source code of the software. This feature requires that the source code be modified each year when the new model year data become available. As a result, the entire program must be recompiled each year.

\section{Approach}

During FY 1996, BFRL will assist NIST's Office of Law Enforcement Standards in preparing the data files compatible with AutoBid for the 1996 model year. BFRL will begin work on redesigning the software to recognize automatically any vehicle model year data files in the current directory and give the user a menu to select any given year for which the data are present.

\section{Recent Results}

Weber, Stephen E., AutoBid 2.0: The Microcomputer System for Police Patrol Vehicle Selection, NISTIR 5787, National Institute of Standards and Technology, February 1996. 


\section{Decision Support Software for Hospitals Financed by HUD- Guaranteed Loans}

\author{
Principal Investigator \\ Stephen F. Weber \\ Office of Applied Economics \\ 301-975-6137
}

\section{Sponsor}

U.S. Department of Health and Human

Services

Health Resources and Services Administration

\section{Objective}

To develop a comprehensive decision support software system for the hospitals and health care facilities financed by HUD-guaranteed loans.

\section{Problem}

The Division of Facilities and Loans of the Health Resources and Services Administration (HRSA) manages a large portfolio of HUDguaranteed loans made to hospitals and health care facilities. Existing software is used solely for manual recording and reporting on the status of outstanding loans and does not support optimization of decisions on operation, maintenance, repair, and expansion expenditures over the life cycle of the facilities. Moreover, the system is not integrated with other critical HUD data about the current condition of the hospital facilities themselves. The source code is unavailable, so that the software cannot be modified or updated. HRSA sponsors need a new system that can access all relevant data to support critical decisions, such as when to survey hospitals for compliance with the Life Safety Code, when an energy conservation audit is advisable, and whether to renew or refinance a loan because of the facility's operation and maintenance performance.

\section{Approach}

During FY 1996, (first phase of this project) BFRL will first evaluate the existing sof tware and interview users to specify data elements and user requirements in terms of functions and reports. BFRL also will review all HUD data used for supporting operation, maintenance, repair, and expansion decisions about the facilities. A draft data dictionary of all files and data fields will be developed and a summary of user requirements produced. It will be submitted for approval by HRSA sponsors. A prototype of the system then will be developed. Once the prototype software is approved by HRSA, a working test version of the data entry and modules reviewed. A standardized report module for the monthly Loan Status Manual will be developed. After the test version of the sof tware has been evaluated by HRSA, BFRL will revise it per comments and integrate the report module into it. Finally, the sof tware will be delivered to HRSA, installed, and import the existing data into the new software.

During Phase 2, BFRL will participate in a study to determine the appropriateness and feasibility of read-only remote access to the data by HUD Hospital Mortgage Insurance Staff as a substitute for the existing centrally printed quarterly reports. Such access at the local level will make possible more cost-effective decisions about the health care facilities. Phase 3 will involve development of the necessary modules to permit customized, user-defined queries of the data. We will also develop decision support modules to guide actions on major expenditures for repairs, renovations, and expansions of the health care facilities.

\section{Recent Results}

New project 


\section{Economic Support for BFRL}

\section{Principal Investigator}

Harold E. Marshall

Office of Applied Economics

301-975-6131

\section{Sponsor}

National Institute of Standards and Technology

\section{Objective}

To implement the comprehensive plan for the Office of Applied Economics's (OAE) support of BFRL's goals and contribute to the revision of old and development of new standard economic methods, guides, and classifications to help the national and international building communities evaluate new technologies and achieve affordable buildings that meet performance objectives.

\section{Problem}

The building community needs sophisticated, practical methods and guidelines for evaluating alternative building technologies in a consistent manner. The need for standardized, improved methods and guidelines stems in large part from the high costs of building materials, the high costs of construction due to safety/environmental regulations, the alleged slow growth in construction productivity, the uncertain costs of energy, and the uncertain lifecycle costs associated with new technologies.

\section{Approach}

During FY 1996, OAE will update and implement the comprehensive plan/vision for economic analysis in support of BFRL's goals. The plan includes a vision, values, mission, and goals for OAE in support of BFRL and NIST. It includes proposed projects such as standard methods for measuring the cost effectiveness of new technologies (for example, composite materials); standard methods and supporting computer programs for promulgation through ASTM (multi attribute decision analysis standard and software); support to the National Science and Technology Council's Subcommittee on Construction and Buildings $(C \& B)$; and recruitment targets and staff assignments to achieve the plan.
Economic methods and supporting analyses will be specially developed for application to building problems. The principal avenues for transferring them to the building community will be the ASTM Building Economics Subcommittee, the C\&B Subcommittee, and the CIB Building Economics Working Commission. For the latter, technical and administrative support will be provided CIB's Working Commission through continuing work on the Spon book on project evaluation. It will be the first volume of the CIB-sponsored Building Economics Reader Series.

Administrative and technical leadership will be provided the ASTM Subcommittee. In FY 1996, the major technical effort will be completing the successful balloting of a new standard method for multi attribute decision analysis (MADA) and developing models of building and materials appiications for use in an ASTM Software Product being developed to support the standard. OAE will continue to chair the Building Economics Subcommittee and perform technical work that contributes to new and revised standards.

\section{Recent Results}

Norris, Gregory A., and Marshall, Harold E., Multi attribute Decision Analysis Method for Evaluating Buildings and Systems, NISTIR 5663, National Institute of Standards and Technology, September 1995.

Marshall, Harold E., Least-Cost Energy Decisions for Buildings -- Part III: Choosing Economic Evaluation Methods Video Training Workbook, NISTIR 5604, National Institute of Standards and Technology, May 1995.

Marshall, Harold E., "Roundtable on a Standard Method for Value Engineering: AACE International Annual Meeting June 21, 1994," Cost Engineering, Vol. 37, No. 3, pp. 9-21, March 1995. 


NGT 